\title{
Progressive Collapse Analysis of Reinforced Concrete Bridges during Earthquakes
}

\author{
by \\ Hartanto Wibowo \\ S.T. \\ Petra Christian University, Surabaya, East Java, Indonesia
}

A thesis submitted to

the Faculty of Graduate Studies and Research

in partial fulfillment of the requirements for the degree of

Master of Applied Science

Department of Civil and Environmental Engineering

Carleton University

Ottawa, Ontario, Canada

August 2009

The Master of Applied Science Program in Civil Engineering is a Joint Program with the University of Ottawa

and Administered by the Ottawa-Carleton Institute for Civil Engineering

Copyright $(2009$ Hartanto Wibowo

All Rights Reserved 
Library and Archives

Canada

Published Heritage Branch

395 Wellington Street

Ottawa ON K1A ON4

Canada
Bibliothèque et

Archives Canada

Direction du

Patrimoine de l'édition

395 , rue Wellington

Ottawa ON K1A ON4

Canada
Your file Votre refférence

ISBN: 978-0-494-60235-5

Our file Notre référence

ISBN: 978-0-494-60235-5
NOTICE:

The author has granted a nonexclusive license allowing Library and Archives Canada to reproduce, publish, archive, preserve, conserve, communicate to the public by telecommunication or on the Internet, loan, distribute and sell theses worldwide, for commercial or noncommercial purposes, in microform, paper, electronic and/or any other formats.

The author retains copyright ownership and moral rights in this thesis. Neither the thesis nor substantial extracts from it may be printed or otherwise reproduced without the author's permission.
AVIS:

L'auteur a accordé une licence non exclusive permettant à la Bibliothèque et Archives Canada de reproduire, publier, archiver, sauvegarder, conserver, transmettre au public par télécommunication ou par l'Internet, prêter, distribuer et vendre des thèses partout dans le monde, à des fins commerciales ou autres, sur support microforme, papier, électronique et/ou autres formats.

L'auteur conserve la propriété du droit d'auteur et des droits moraux qui protège cette thèse. $\mathrm{Ni}$ la thèse ni des extraits substantiels de celle-ci ne doivent être imprimés ou autrement reproduits sans son autorisation.
In compliance with the Canadian Privacy Act some supporting forms may have been removed from this thesis.

While these forms may be included in the document page count, their removal does not represent any loss of content from the thesis.
Conformément à la loi canadienne sur la protection de la vie privée, quelques formulaires secondaires ont été enlevés de cette thèse.

Bien que ces formulaires aient inclus dans la pagination, il n'y aura aucun contenu manquant. 
Department of Civil and Environmental Engineering

\title{
Progressive Collapse Analysis of Reinforced Concrete Bridges during Earthquakes
}

\author{
Submitted by \\ Hartanto Wibowo \\ in partial fulfillment of the requirements for the degree of \\ Master of Applied Science
}


Dedicated to my parents 


\section{Acknowledgments}

The author would like to express his gratitude to Professor David T. Lau for his supervision, advice, and support from the very early stages of this research as well as for his constant motivation throughout the work. He has been a great mentor and his inspirational thoughts and passions in science will always be remembered and enrich the author's growth as a student, researcher, and scientist. It has been a pleasure to work with a person like him and hopefully this will continue in future collaborations.

The author would also like to thank all faculty members and staffs in the Department of Civil and Environmental Engineering under the chairmanship of Professor A. O. Abd El Halim for their assistance and their contribution to the author's intellectual development.

The author wishes to thank Dr. Hatem Tagel-Din and Mr. Ayman El-Fouly of the Applied Science International, LLC for providing the technical support and fruitful discussion of the software ELS. The author is also indebted to Professor Rui Pinho of the University of Pavia for his assistance in providing the bridges' reinforcement details.

The author is also grateful to his colleagues in the Ottawa - Carleton Bridge Research Institute; in particular to Ryan F. O'Connell, Charlotte L. Waller, and 
Silvena S. Reshotkina, also all graduate students in the department especially Seyyed Morteza Mesbah, Audrey Murray, Farrokh Fazileh, Richard Michels, Maryam Mirzajani, and Alain-Philippe Beauger for their support and kind help whenever needed and for creating a friendly atmosphere during his stay at Carleton University. Their friendship has made Ottawa a home away from home for the author.

Last but not least, special thanks go to the family members who always unconditionally supported and encouraged the author throughout the course of this study. Without them this thesis would not be possible. 


\begin{abstract}
Previous research on progressive collapse of structures has mainly focused on blast and/or abnormal loadings. Progressive collapse behaviour of structures, especially bridges, caused by earthquake loadings is not as well understood. This lack of information is also reflected in the lack of specific provisions or recommendations in current codes and standards on seismic design of structures. In order to design safer structures against earthquakes and to come up with more cost effective retrofit strategies for the vast number of existing old and often deteriorated bridges in today's bridge stock, it is important to have a complete understanding of the progressive collapse behaviour of structures from initial failure of individual structural members, progression of the damage and degradation of stiffness and strength, to ultimate collapse of the system.
\end{abstract}

This thesis presents results of a study on earthquake response analysis of bridges by using the Applied Element Method that allows separation of structural members or components due to fracture failure, and the effects of contact and inertial forces caused by the falling debris. The analysis is able to predict the initiation of collapse, progression of collapse, and the final collapse mechanisms. Understanding of the progressive collapse behaviour of structures can lead to a better and more effective design and retrofit strategy for earthquake resistant design of structures. The results show that collapse of single structural component of the bridge can have 
significant ramification effect that can drastically change the behaviour of the entire bridge system in a process that is not well understood before. The analysis outputs also give information on the structure's condition during and after earthquakes so that more effective performance-based design retrofit strategies can be devised by taking into account the expected damage of the structure. 


\section{Table of Contents}

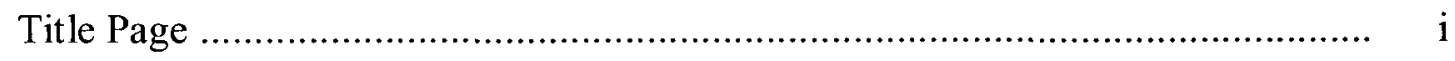

Acceptance Page ........................................................................ ii

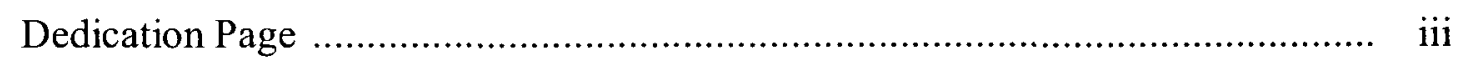

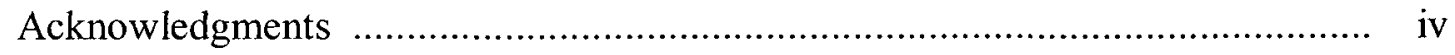

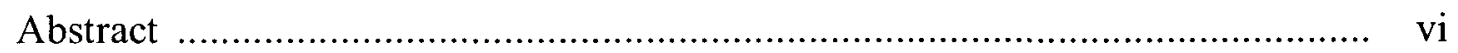

Table of Content ............................................................................. viii

List of Tables ...............................................................................

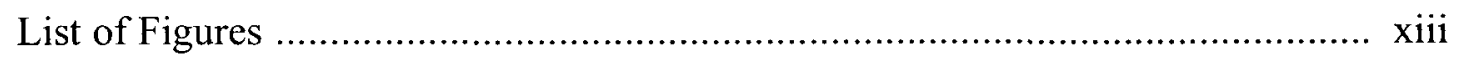

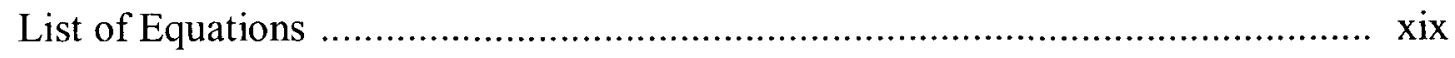

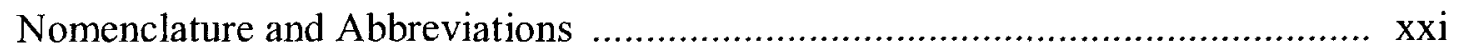

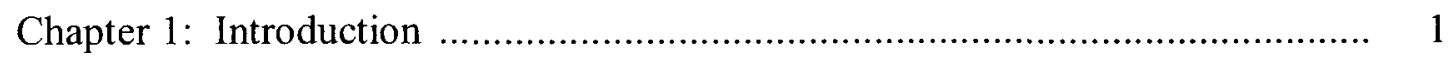

1.1. General Background ........................................................ 1

1.2. Problem Statement ....................................................... 5

1.3. Objectives and Scope ...................................................... 6

1.4. Research Methodology ......................................................... 7

1.5. Thesis Outline ................................................................ 9

Chapter 2: Literature Review .................................................... 11

2.1. Definition of Progressive Collapse .................................... 11

2.2. Structural Resistance to Progressive Collapse ........................... 13

2.3. Progressive Collapse Design of Buildings ................................ 15

2.4. Analysis Methods for Progressive Collapse ............................ 20 
2.5. Earthquake Loads and Progressive Collapse .................................. 23

2.6. Progressive Collapse of Bridges ................................................... 25

2.6.1. Progressive Collapse Analysis of Bridges ........................... 26

2.6.2. Progressive Collapse Resistant Design of Bridges ................ 28

2.7. Analytical Tools .................................................................. 30

2.7.1. Finite Element Method-Based Tools ………........................ 31

2.7.2. Non-Finite Element Method-Based Tools ............................. 34

Chapter 3: Overview of the Applied Element Method .................................... 41

3.1. Theory and Formulation of the Applied Element Method .................. 41

3.1.1. Element Formulation in Static Small Deformation Range ...... 43

3.1.2. Element Formulation in Static Large Deformation Range ...... 46

3.1.3. Element Formulation in Dynamic Small Deformation Range . 49

3.1.4. Element Formulation in Dynamic Large Deformation Range . 52

3.1.5. Eigenvalue Analysis ...................................................... 55

3.1.6. Material Modelling .......................................................... 55

3.1.7. Failure Criteria _.............................................................. 59

3.1.8. Element Formulation for Collision and Re-contact ............... 64

3.1.9. Effects of Number of Connecting Springs between Elements . 68

3.1.10. Effects of Element Size …………………....................... 69

3.1.11. Effects of Poisson's Ratio .................................................... 69

3.2. Application of the Applied Element Method ………...................... 71

3.2.1. Element Generation ........................................................ 71

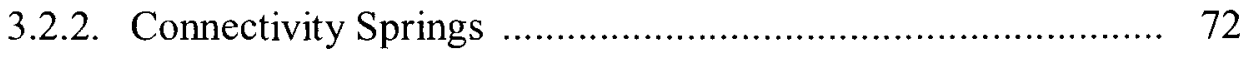

3.2.3. Material Properties ......................................................... 74 
3.2.4. Contact Parameters ......................................................... 77

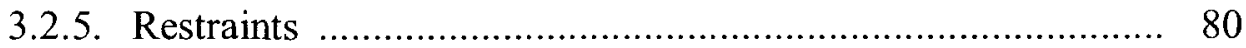

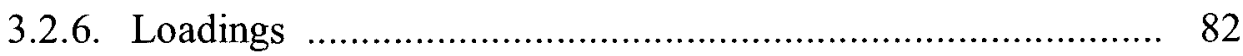

3.2.7. Analysis Solver …......................................................... 83

Chapter 4: Bridge Models and Ground Accelerations ...................................... 109

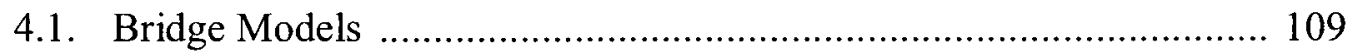

4.1.1. Overview of the Short-Span Bridge Model …………........... 110

4.1.2. Overview of the Medium-Span Bridge Model ...................... 112

4.1.3. Transverse Reinforcement ……………………….............. 113

4.2. Ground Acceleration .............................................................. 114

4.2.1. Artificial Ground Acceleration from Literature ..................... 115

4.2.2. Modified Kobe Earthquake Ground Acceleration ................. 116

4.2.3. Northridge Earthquake Ground Acceleration ........................ 116

4.2.4. Kobe Earthquake Ground Acceleration …............................. 117

4.2.5. Chi-Chi Earthquake Ground Acceleration ............................ 117

Chapter 5: Results and Discussion ............................................................ 137

5.1. Verification Examples ……………………............................... 137

5.1.1. Frame Model .................................................................. 138

5.1.2. Verification Results ....................................................... 138

5.1.3. Effects of Point and Distributed Loads ................................ 139

5.2. Seismic Progressive Collapse of Bridges with Minimum Box Girder

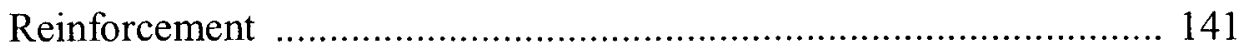

5.2.1. Displacements ............................................................... 141

5.2.2. Internal Forces .............................................................. 142 
5.3. Parametric Study

5.3.1. Effects of Dynamic Properties and Deterioration of the Structure 143

5.3.2. Effects of Earthquake Ground Motion 146

5.4. Evaluation of the Assumption of Elastic Superstructure Model 147

5.4.1. Short-Span Bridge Model 148

5.4.2. Results for Elastic Superstructure Model 149

5.4.3. Results for Regular Superstructure Model 151

5.5. Discussion 152

Chapter 6: Conclusions and Recommendations 205

6.1. Conclusions Remarks 205

6.1.1. Findings on Seismic Progressive Collapse of Bridges 206

6.1.2. Findings on the Applicability of the Applied Element Method for Seismic Progressive Collapse Analysis of Structures 208

6.2. Suggestion for Future Works 209

6.2.1. Further Research on Seismic Progressive Collapse of Bridges 210

6.2.2. Further Development of the Software Extreme Loading for Structures

References 213 


\section{List of Tables}

Table 4.1. Concrete Pier Properties of the Short-Span Bridge Model .............. 118

Table 4.2. Steel Properties of the Short-Span Bridge Model .......................... 119

Table 4.3. Concrete and Steel Properties of the Medium-Span Bridge Model .. 120

Table 4.4. Summary of the Earthquake Ground Motions …............................. 121

Table 5.1. Properties of the Verification Model .............................................. 155

Table 5.2. Comparison of Vibration Periods .................................................. 156

Table 5.3. Comparison of Viaduct Fundamental Periods from Different

Combinations

Table 5.4. First Three Modal Periods and Shapes in the Transversal

Direction of the Box Girder from the Literature

Table 5.5. First Three Modal Periods and Shapes in the Transversal

Direction of the Box Girder from ELS 


\section{List of Figures}

Figure 2.1. Collapses of (a) Ronan Point Tower, (b) Alfred P. Murrah Building, and (c) World Trade Center

Figure 2.2. Examples of Maximum Allowable Collapse Area in GSA

Guidelines

Figure 2.3. Tie Forces Described in UFC 4-023-03

Figure 2.4. Collapse of Bridges during the (a) 1964 Niigata, (b) 1989 Loma

Prieta, and (c) 1995 Kobe Earthquakes

Figure 2.5. Progressive Collapse Resistant Design Applied on the

Confederation Bridge

Figure 3.1. Analysis Domain of the Applied Element Method

Figure 3.2. Elements on the Applied Element Method

Figure 3.3. Element Contact Point and Degrees of Freedom 86

Figure 3.4. Normal Springs for Rotational Stiffness Calculation

Figure 3.5. Maekawa Concrete Model 88

Figure 3.6. Model for Concrete under Shear Stress 89

Figure 3.7. Ristic Steel Model 90

Figure 3.8. Principal Stresses for 2D Problems 91

Figure 3.9. Strategies for Element Cracking 92

Figure 3.10. Principal Stresses for 3D Problems 93 
Figure 3.11. (a) Collision of Elements and (b) Springs Generated during Collision

Figure 3.12. Elements in ELS ................................................................ 95

Figure 3.13. Partial Connectivity in (a) FEM and (b) AEM ............................. 96

Figure 3.14. Comparison of Mesh in (a) FEM and (b) AEM ............................ 97

Figure 3.15. (a) 3D Element's Degrees of Freedom and (b) Deformations Related to the Degrees of Freedom ……....................................... 98

Figure 3.16. Matrix Springs between Elements ............................................... 99

Figure 3.17. Rebar Springs between Elements ................................................. 100

Figure 3.18. Illustration of Young's Modulus of (a) Concrete and (b) Steel ........ 101

Figure 3.19. Illustration of Shear Modulus ...................................................... 102

Figure 3.20. Illustration of Yield and Ultimate Stresses of Rebar ..................... 103

Figure 3.21. Illustration of Separation Strain .................................................. 104

Figure 3.22. Illustration of Element Separation and Re-contact ......................... 105

Figure 3.23. Illustration of Element Friction ……............................................ 106

Figure 3.24. Illustration of Post-yield Stiffness Ratio ........................................ 107

Figure 3.25. Illustration of Contact Spring Unloading Stiffness Factor .............. 108

Figure 4.1. Short-Span Bridge Elevation and Sections ................................. 122

Figure 4.2. 3D View of the Short-Span Bridge Model in ELS ........................ 123

Figure 4.3. Box Girder's Longitudinal Reinforcement Details ......................... 124

Figure 4.4. (a) Type 1 and (b) Type 2 Piers' Longitudinal Reinforcement

Details 125

Figure 4.5. Cap on Top of the Pier to Model Pin Connection ............................ 126

Figure 4.6. Medium-Span Bridge Elevation and Sections .............................. 127 
Figure 4.7. 3D View of the Medium-Span Bridge Model in ELS 128

Figure 4.8. Artificial Earthquake Ground Motion from the Thesis by Guedes ... 129

Figure 4.9. (a) X-, (b) Y-, and (c) Z-Acceleration Components of the Modified Kobe Earthquake Ground Motion

Figure 4.10. (a) X-, (b) Y-, and (c) Z-Acceleration Components of the 1994 Northridge Earthquake Ground Motion

Figure 4.11. (a) X-, (b) Y-, and (c) Z-Acceleration Response Spectra of the 1994 Northridge Earthquake Ground Motion

Figure 4.12. (a) X-, (b) Y-, and (c) Z-Acceleration Components of the 1995 Kobe Earthquake Ground Motion 133

Figure 4.13. (a) X-, (b) Y-, and (c) Z-Acceleration Response Spectra of the 1995 Kobe Earthquake Ground Motion

Figure 4.14. (a) X-, (b) Y-, and (c) Z-Acceleration Components of the 1999 Chi-Chi Earthquake Ground Motion 135

Figure 4.15. (a) X-, (b) Y-, and (c) Z-Acceleration Response Spectra of the 1999 Chi-Chi Earthquake Ground Motion 136

Figure 5.1. 2D View of Verification Model in (a) ELS and (b) SAP2000 160

Figure 5.2. Linear Analyses Verification Results: (a) Displacement,

(b) Moment, (c) Shear Force, (d) Axial Force 161

Figure 5.3. Nonlinear Analyses Verification Results: (a) Displacement,

(b) Moment, (c) Shear Force, (d) Axial Force 163

Figure 5.4. First Six Modal Shapes of the Verification Model 165

Figure 5.5. Location of the (a) Point and (b) Distributed Loads 166 
Figure 5.6. Deformations of Elements Caused by the (a) Point and

(b) Distributed Loads

Figure 5.7. (a) Locations and (b) the Positive Global Axes of the

Measured Elements 168

Figure 5.8. (a) X-, (b) Y-, and (c) Z-Displacements of the Elements Caused by the Point Load

Figure 5.9. (a) X-, (b) Y-, and (c) Z-Displacements of the Elements Caused by the Distributed Load 170

Figure 5.10. Progression of Collapse of Bridge with Minimum Box Girder Reinforcement

Figure 5.11. Displacement Time History along Z-Axis of the Middle Element of Deck E-F 172

Figure 5.12. (a) Displacement Time History of the Non-Falling Element and (b) Comparison between the Non-Falling and Falling Elements along X-Axis at the Top of Pier F 173

Figure 5.13. Overturning Moment Time History about Y-Axis at the Base of

Piers $\mathrm{E}$ and $\mathrm{F}$ 174

Figure 5.14. Shear Force Time History about X-Axis at the Base of Piers $\mathrm{E}$ and $\mathrm{F}$ 175

Figure 5.15. Axial Force Time History at the Base of Piers E and F 176

Figure 5.16. (a) Elevation and (b) 3D Views of the Viaduct Model 177

Figure 5.17. Two Types of Viaduct's Beam: (a) B1 and (b) B2 178

Figure 5.18. Two Types of Viaduct's Column: (a) C1 and (b) C2 179

Figure 5.19. Structural Deformations of Viaduct B2C2-B 180 
Figure 5.20. Structural Deformations of Viaduct B2C2-C

Figure 5.21. (a) Top Displacement, (b) Overturning Moment, (c) Shear Force, and (d) Axial Force Time Histories for Viaducts B2C2-B and $\mathrm{B} 2 \mathrm{C} 2-\mathrm{C}$

Figure 5.22. Structural Deformations of Viaduct B2C1-C 184

Figure 5.23. Structural Deformations of Viaduct B1C2-C 185

Figure 5.24. (a) Top Displacement, (b) Overturning Moment, (c) Shear Force, and (d) Axial Force Time Histories for Viaducts B1C1-C, $\mathrm{B} 1 \mathrm{C} 2-\mathrm{C}, \mathrm{B} 2 \mathrm{C} 1-\mathrm{C}$, and $\mathrm{B} 2 \mathrm{C} 2-\mathrm{C}$ 186

Figure 5.25. Structural Deformation of Short-Span Bridge due to the 1994 Northridge Earthquake Excitation 188

Figure 5.26. Structural Deformation of Short-Span Bridge due to the 1995 Kobe Earthquake Excitation 189

Figure 5.27. Structural Deformation of Short-Span Bridge due to the 1999 Chi-Chi Earthquake Excitation 190

Figure 5.28. (a) Short, (b) Medium, and (c) Tall Piers' Top Displacement Time Histories along the X-Axis due to the 1994 Northridge Earthquake Excitation 191

Figure 5.29. (a) Short, (b) Medium, and (c) Tall Piers' Top Displacement Time Histories along the X-Axis due to the 1995 Kobe Earthquake Excitation 192

Figure 5.30. (a) Short, (b) Medium, and (c) Tall Piers' Top Displacement Time Histories along the X-Axis due to the 1999 Chi-Chi Earthquake Excitation 
Figure 5.31. Dislocation and Cracks of the Short Pier due to the 1995 Kobe Earthquake Excitation

Figure 5.32. (a) Moment, (b) Shear Force, and (c) Axial Force Time Histories at the Base of the Piers due to the 1994 Northridge Earthquake Excitation 195

Figure 5.33. (a) Moment, (b) Shear Force, and (c) Axial Force Time Histories at the Base of the Piers due to the 1995 Kobe Earthquake Excitation

Figure 5.34. (a) Moment, (b) Shear Force, and (c) Axial Force Time Histories at the Base of the Piers due to the 1999 Chi-Chi Earthquake Excitation 197

Figure 5.35. Types of Abutments: (a) A1, (b) A2, and (c) A3 198

Figure 5.36. Box Girder Rocking Modal Shape 199

Figure 5.37. Comparisons of (a) Short, (b) Medium, and (c) Tall Piers'

Displacements with Elastic Superstructure Model 200

Figure 5.38. Comparisons of (a) Short, (b) Medium, and (c) Tall Piers' Top Shear Forces with Elastic Superstructure Model 201

Figure 5.39. Progression of Collapse of Short-Span Bridge Model with Regular Superstructure Model 202

Figure 5.40. Comparisons of (a) Short, (b) Medium, and (c) Tall Piers' Displacements with Regular Superstructure Model 203

Figure 5.41. Comparisons of (a) Short, (b) Medium, and (c) Tall Piers'

Top Shear Forces with Regular Superstructure Model 204 


\section{List of Equations}

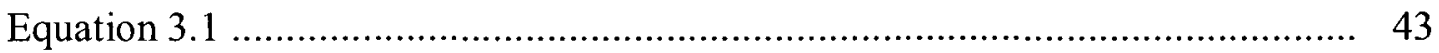

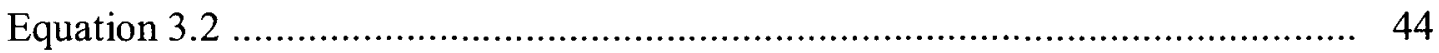

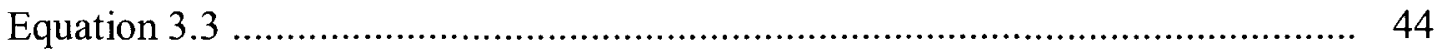

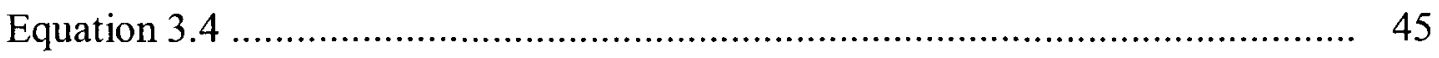

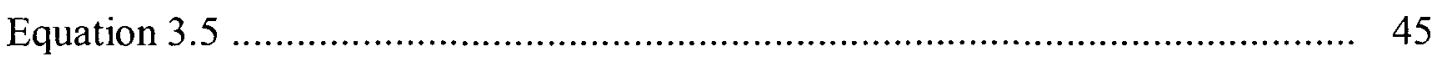

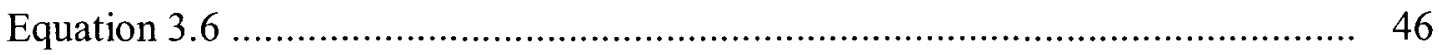

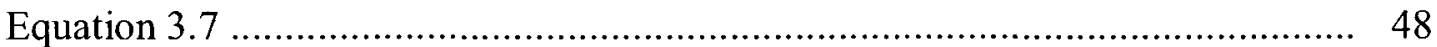

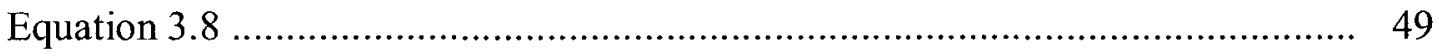

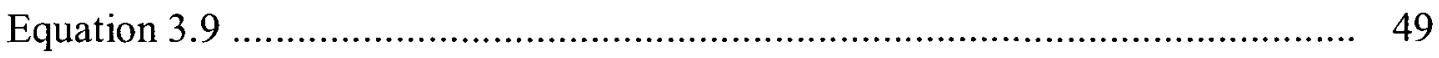

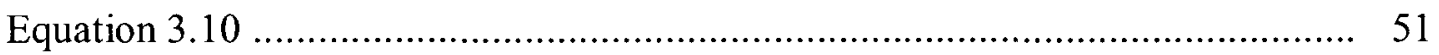

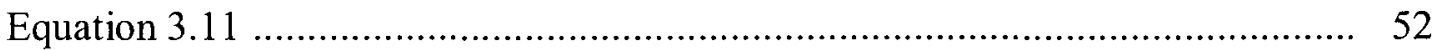

Equation 3.12 ............................................................................ 53

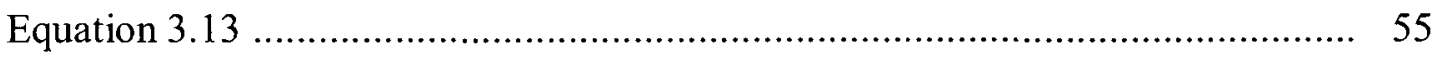

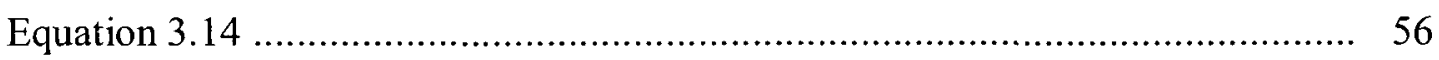

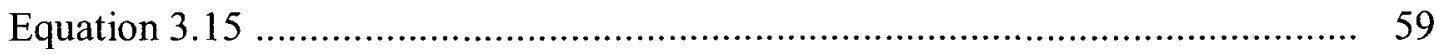

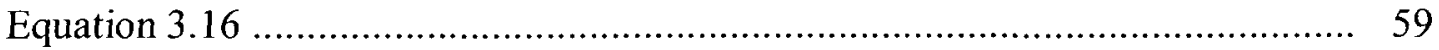

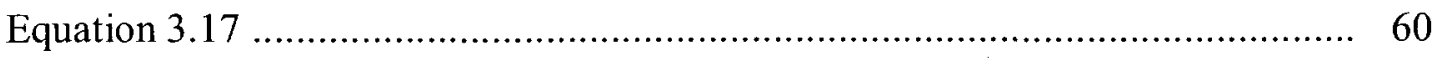

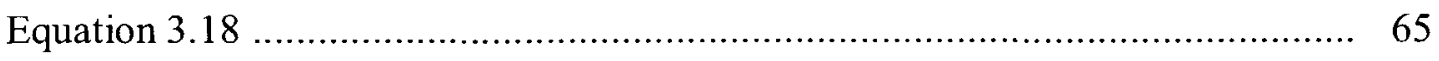

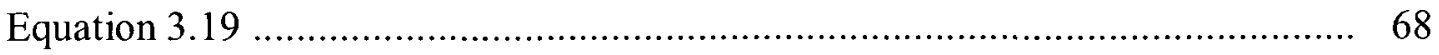

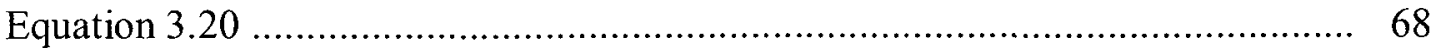




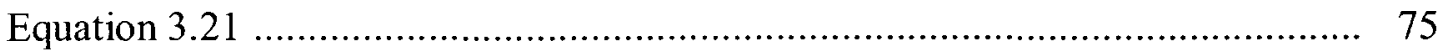

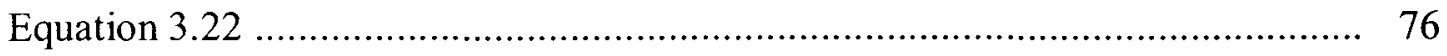

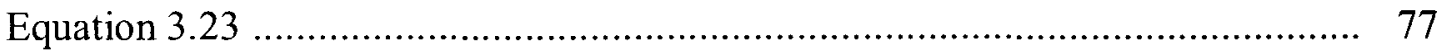

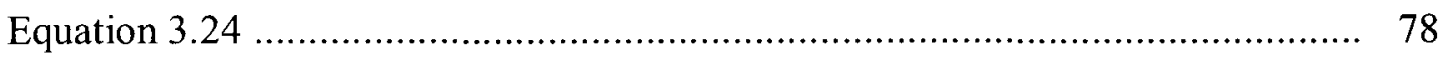

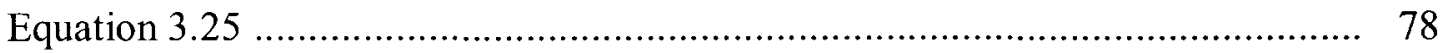

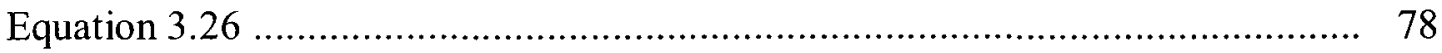

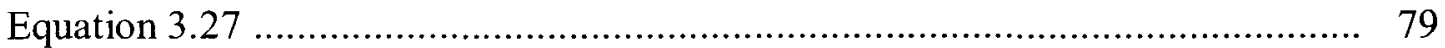




\title{
Nomenclature and Abbreviations
} \\ Length of the representative area or element width \\ $b$ \\ Element height \\ $d$ \\ Distance between springs \\ $f$ \\ External applied force vector \\ G Gravitational constant \\ $\mathrm{f}_{\mathrm{c}}$ \\ Compressive strength from the stress-strain relationship \\ $f_{c, e q}$ \\ Modified compressive strength \\ $i$ \\ Spring number \\ $n$ \\ Half of the number of springs \\ $n$ \\ Contact spring unloading stiffness factor \\ $r \quad$ Damping factor \\ $t$ \\ Element thickness \\ $\mathrm{x}$ \\ Distance from the element perimeter to point $\mathrm{A}$ \\ Distance from the spring to the centroid
}




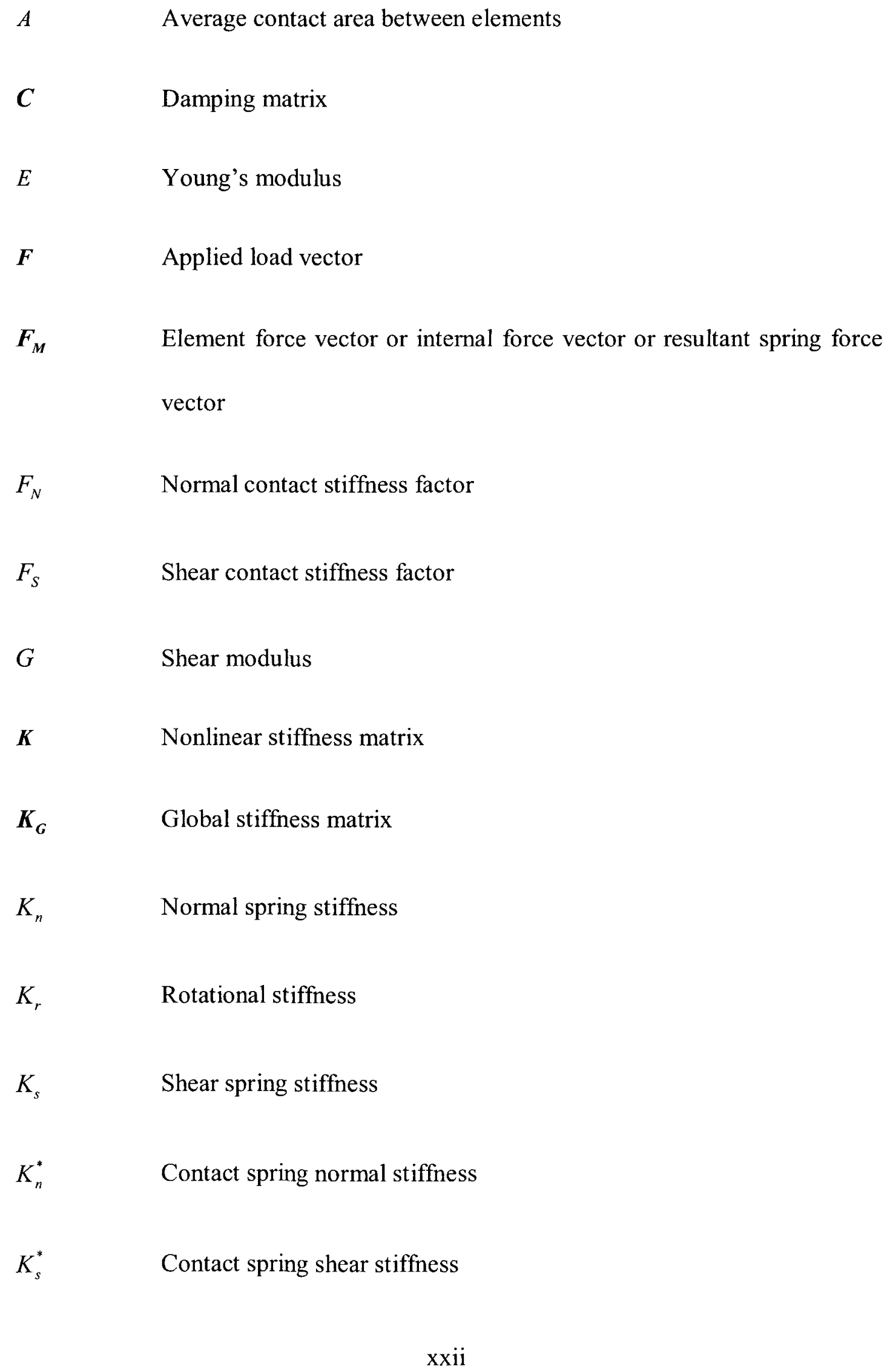


$L \quad$ Distance from the location of the contact spring to the centroid of the rigid element

$M \quad$ Mass matrix

$M_{1} \quad$ Element mass corresponding to DOFs $\mathrm{u}_{1}$

$M_{2} \quad$ Element mass corresponding to DOFs $\mathrm{u}_{2}$

$M_{3} \quad$ Element moment of inertia around the centroid corresponding to DOF $\mathrm{u}_{3}$

$\boldsymbol{R}_{\boldsymbol{G}} \quad$ Residual force vector due to geometrical changes of the structure during loading

$\boldsymbol{R}_{M} \quad$ Residual force vector due to cracking or incompatibility between strains and stresses at the spring location (nonlinear behaviour of the material)

$T \quad$ Natural period

$\boldsymbol{U} \quad$ Displacement vector

$\alpha$

Orientation angles of the rigid element defined in Figure 3.3

$\beta \quad$ Local crack inclination angle to the element edge direction

$\theta \quad$ Orientation angles of the rigid element defined in Figure 3.3 


\begin{tabular}{|c|c|}
\hline$v$ & Poisson's ratio \\
\hline$\xi$ & Damping ratio \\
\hline$\rho$ & Density of the element's material \\
\hline$\sigma_{1}$ & Normal stress \\
\hline$\sigma_{2}$ & Secondary stress \\
\hline$\sigma_{\mathrm{p}}$ & Principal stress \\
\hline$\sigma_{\mathrm{B}}$ & Normal stress at point B \\
\hline$\sigma_{\mathrm{C}}$ & Normal stress at point $C$ \\
\hline$\tau$ & Shear stress \\
\hline$\omega$ & Eigenvalue vector or natural frequency \\
\hline$\omega_{1}$ & First natural frequency of the structure \\
\hline$\Delta f$ & Incremental applied load vector \\
\hline$\Delta f(t)$ & Incremental applied load vector for each time step \\
\hline$\Delta f_{S}$ & Incremental static load vector \\
\hline$\Delta \boldsymbol{U}$ & Incremental displacement vector \\
\hline
\end{tabular}


$\begin{array}{ll}\Delta \dot{U} & \text { Incremental velocity vector } \\ \Delta \dot{U} & \text { Incremental acceleration vector } \\ \Delta \ddot{U}_{g} & \text { Incremental ground acceleration vector }\end{array}$

AASHTO American Association of State Highway and Transportation Officials

$\mathrm{ACI} \quad$ American Concrete Institute

AEM Applied Element Method

ASCE American Society of Civil Engineers

ASI Adaptively Shifted Integration

ASI Applied Science International, LLC

CSA Canadian Standards Association

DEM Discrete (or Distinct) Element Method

DoD Department of Defense

DOF Degree-of-Freedom

EDEM Extended Discrete Element Method

ELS Extreme Loading for Structures

F/DEM Finite - Discrete Element Method 


$\begin{array}{ll}\text { FEM } & \text { Finite Element Method } \\ \text { FEMA } & \text { Federal Emergency Management Agency } \\ \text { GSA } & \text { General Services Administration } \\ \text { MDEM } & \text { Modified Discrete Element Method } \\ \text { NIST } & \text { National Institute of Standards and Technology } \\ \text { NRCC } & \text { National Research Council of Canada } \\ \text { PBEE } & \text { Performance-Based Earthquake Engineering } \\ \text { PEER } & \text { Pacific Earthquake Engineering Research } \\ \text { RBSM } & \text { Rigid Body and Spring Model } \\ \text { RV } & \text { Redistributed Value } \\ \text { XFEM } & \text { Extended Finite Element Method }\end{array}$




\section{CHAPTER 1}

\section{Introduction}

This chapter serves as the introductory part of the thesis. Background of the research, problem statement, objectives and scope, methodology, and the thesis outline are discussed.

\subsection{General Background}

Earthquakes have been the cause of many devastating events in the world's history. In modern times, besides the tremendous loss of lives as a result of the direct effects of earthquakes such as structural collapse and damage or tsunami, the aftermaths of an earthquake in or near an urban area can also include significant economic losses in properties and infrastructures as well as disruption to economic activities. In earthquake resistant design of structures, it has been long established that the generally accepted design objective of typical structures is life safety. Recently in the new development of the Performance-Based Earthquake Engineering (PBEE), the aim in seismic design of structures has evolved, in addition to the life safety performance objective, to include also consideration of minimizing economic loss and maintaining continuous operation and service during or shortly after an 
earthquake event. In performance-based seismic design, a structure is designed not only to be safe but also disaster resilient in order to minimize post-earthquake disruptions to its operation and services provided to its users or occupants. In the PBEE, these goals can be addressed in terms of expected downtime of a structure after an earthquake, the amount of structural damage sustained, and cost and time required to restore operation and services. To achieve the goals in seismic performance of structures in the PBEE, it is necessary to have a thorough understanding of the complete structural response behaviour over the entire duration of seismic response from initial failure of individual structural components or members, progression of structural failure, and the influence of accumulated structural damage on the strength and stiffness of the structure to the total collapse of the structural system. Also, accurate prediction models and analysis tools are needed for determining the complete detailed inelastic behaviour and performance of structures during earthquakes.

In recent years, progressive collapse behaviours of structures have received considerable increased attention by structural engineers especially after several major incidents of building collapses caused by blast and impact loads. Although most recent studies on progressive collapse of structures have focused on blast load effects on structures, the observations and findings from these studies provide valuable insights to progressive collapse behaviour of structures during earthquakes. In cases of failure caused both by blast and earthquake loads, structural failure is initiated due to overstress and load exceeding the strength capacity of individual components or 
members of a structure followed by propagation of the damage to adjacent supporting members due to load redistribution and the redistributed loads exceeding the capacity of the adjacent structural members, which subsequently leads to partial or total collapse of the structural system. From studies of earthquake damage of structures compared to structural damage caused by blast loads, it is observed that the structural damage induced by blast loads tends to be more localized compared to those caused by earthquakes.

With the aging public infrastructure problems becoming increasingly more critical and it has been recognized that many existing public infrastructure of buildings and bridges are deficient in their earthquake resistance, coupled with the demonstrated need for improvement in the current seismic design methodology of structures, research is needed for better understanding of progressive collapse behaviour of structures during earthquakes in order to develop more cost effective retrofit and upgrade solutions and strategies for existing deficient structures and improve seismic performance of performance-based design of new structures. Some considerations of progressive collapse resistance have been introduced in design codes of building in 1970's [Taylor, 1975]. However, these early considerations are mostly qualitative requirements with very little analytical or experimental research to support or justify the validity and effectiveness of these design requirements. The recent recommendations on building design include some guidelines to provide some quantitative requirements for progressive collapse resistant design of buildings against blast loads. On the other hand, the understanding of the significance of 
progressive collapse resistance in overall structural performance and design has not reached the area of bridge design [CSA, 2006; AASHTO, 2007].

In daily life, bridges are important structures which serve the public as important links in transportation infrastructure. As stated by Barker and Puckett [2007], bridges are key elements in a transportation system for three reasons:

1. They control the capacity of the system.

2. They are the most valuable asset in the system with the highest cost per unit distance.

3. They will cause the whole system to fail if they fail.

In a number of post-earthquake reconnaissance studies of structural damage, the seismic performance of many highway systems during past earthquakes was deemed to be not satisfactory [Kawashima, 2000; Wallace et al., 2001; Buckle, 2003; Moehle and Eberhard, 2003; Han et al., 2009]. Priestley et al. [1996] have noted in their study that reinforced and prestressed concrete bridges constructed prior to the 1970's have not performed well during recent major earthquakes. In addition, bridges are often observed to be among the most vulnerable components in a highway network system [Shinozuka et al., 2007]. There is considerable evidence from past earthquakes that bridges designed for seismic resistance in accordance to the design standard requirements at the time of their construction did not perform as expected during past earthquakes. Many of these bridges suffered severe damage or collapse. 
Considering the aforementioned background and recognizing that bridges are important links in highway transportation networks critical to the regional and national economic well-being of Canada and other parts of the world, it becomes obvious that there is a need to improve the state-of-knowledge on the progressive collapse behaviour of bridges in order to have a better understanding of the problem. A better understanding and insights on the seismic performance of bridges can lead to bridge design improvement, disaster risk reduction, and better emergency preparedness. Better design, retrofit, and mitigation strategies can also be developed to help reducing the enormous cost required for renewing the existing old bridge infrastructures.

\subsection{Problem Statement}

Lessons learned from past bridge collapses and damages during earthquakes have shown that better understanding of complete inelastic seismic response behaviour of bridges, especially after individual member failure leading to load redistribution and the influence of accumulated damage on the system's behaviour and ultimate collapse mechanisms of bridge structures. In the current development of the next generation of performance-based design recommendations for earthquake resistance of bridge structures, the key questions that need to be addressed are as follows: 
- What are the typical progressive collapse failure characteristics of bridges during earthquakes?

What are the parameters governing the progressive collapse of bridges during earthquakes?

What are the influences of the common assumption of bridge deck superstructure remaining elastic during seismic response in current conventional seismic design practice to the actual seismic behaviour and performance of bridges as related to progressive collapse phenomena?

\subsection{Objectives and Scope}

The objective of this thesis is to demonstrate the accuracy and versatility of the new simulation technique by the Applied Element Method (AEM) that can follow the response behaviour of reinforced concrete bridges during earthquakes from the initiation of failure, redistribution of loading, progression of damage spread, to final collapse mechanism. The present research is aimed to obtain insights on seismic progressive collapse behaviour of bridges through computer modelling and analysis. This new modelling and analysis tool can be used to generate knowledge and better understanding of progressive collapse behaviour and performance of structures that would help structural engineers on improving seismic design of new bridges and 
developing more effective retrofit and strengthening strategies for old and deficient bridges.

In this thesis, seismic progressive collapse behaviour of reinforced concrete highway bridges is studied. However, as a pilot study, the effects of soil-structure interaction and multiple-support excitations or spatial variability in the ground motion input on the seismic response behaviour of the example bridge structures are not included in this research.

\subsection{Research Methodology}

Experimental research on progressive collapse phenomena is costly and requires large-scale experimental facilities to test structural specimens until complete failure to simulate the entire response history of the test structure from initial failure to final ultimate collapse of the structural system. Since progressive collapse behaviour involves load redistribution during the failure process of damage propagation in the system, it is generally not possible to obtain insights of the process through testing of only structural sub-assemblages. Testing of the entire structural system is required to properly simulate the progressive collapse behaviour of the test

system, which is even more costly and often technically not feasible because of limitations of physical test facilities, although new hybrid experimental techniques have been developed recently to overcome some of the difficulties. 
In the present study, nonlinear analytical models and numerical simulation techniques are chosen as the approach of research methodology. The proper analytical models suitable for numerical simulation of progressive collapse behaviour of bridges must have the capability to model the entire complex structural response process in following the structural behaviour of the initial continuous structural system and any fracture failure and resulting separation of structural members or components from the system including the impact loading effects of falling debris.

In this study, the progressive collapse behaviours of reinforced concrete bridges are analyzed using the AEM with the computer software Extreme Loading for Structure (ELS). This computer software is chosen since it can simulate the detailed progressive collapse phenomena of structures during earthquakes. The AEM can take into account the effects of separation between discrete fractured structural elements and the impact forces resulting from falling debris during collisions or contacts of fractured components during seismic response. The AEM can provide more detailed information needed for assessing the performance of structures according to the new development of the Performance-Based Earthquake Engineering.

Several frame structures are analyzed as verification examples to compare some of the results obtained by the AEM with those obtained by the Finite Element Method (FEM). Two types of bridges, short- and medium-spans, are also studied and analyzed using nonlinear dynamic time history analysis. Last, the progressive collapse behaviour of the bridges and the parameters that affect the response behaviour and the collapse mechanisms are investigated. 


\subsection{Thesis Outline}

This thesis is divided into six chapters. In addition, two appendices are included to elaborate more on the analysis methods used for the bridge analyses in the thesis.

Chapter 1 presents an introduction on the background, problem statement, objectives and scope, and methodology of the research.

Chapter 2 explains the current state-of-knowledge and gives an overview of the design code and standard requirements on progressive collapse as well as the analytical tools available for progressive collapse analysis of structures. Moreover, progressive collapse behaviours of bridges, caused by earthquakes are also discussed.

Chapter 3 gives the theoretical background of the Applied Element Method and a general overview of the application of the method in Extreme Loading for Structures software.

Chapter 4 presents the bridge models selected for numerical study of progressive collapse behaviour. The bridges selected in the study are short- and medium-span bridges. This chapter also presents details of the earthquake ground motion records used for the time history analyses.

Chapter 5 presents and discusses the numerical verification examples and the results of the study. A simple one-bay frame is analyzed to verify the results for linear 
and nonlinear responses by the AEM and the FEM. In addition, the progressive collapse phenomena of bridges during earthquakes and the factors that can affect the behaviours are discussed.

Chapter 6 presents conclusions and a summary of the findings in this thesis and includes recommendations for future works. 


\section{CHAPTER 2}

\section{Literature Review}

In this chapter the current state-of-knowledge on progressive collapse behavior of structures, an overview of design code and standard requirements of progressive collapse resistant design, as well as the analytical tools available for progressive collapse analysis of structures are presented.

\subsection{Definition of Progressive Collapse}

Progressive collapse of structures may be caused by different load effects such as blasts, earthquakes, or other accidental extreme loads. Depending on the cause of overload, the definition of progressive collapse may be interpreted differently by different researchers. But essentially, progressive collapse of a structure is a phenomenon in which an initial local failure spreads from element to element and eventually results in the collapse of the whole structure or to an extent disproportionate to the original failure. Some researchers distinguish between the terms "progressive collapse" and "disproportionate collapse". Progressive collapse is the collapse of all or a large part of a structure precipitated by damage or failure of a 
relatively small part of it and disproportionate collapse is structural collapse disproportionate to the cause [Nair, 2006].

Progressive collapse or cascading collapse is a catastrophic structural failure mechanism. It first drew the attention of structural engineers after the accidental collapse of the 22-story Ronan Point apartment tower in Canning Town, U.K. on May 16, 1968 [Griffiths et al., 1968]. The cause of the collapse was a human-error gas explosion that knocked out the precast concrete panels near the corner on the $18^{\text {th }}$ floor. The failure of that support caused the floors above to collapse. Since then, building codes in many countries have been updated to include regulations to prevent this type of progressive collapse behaviour. Following nearly three decades of relatively few developments related to progressive collapse issues, a significant and much publicized case of progressive collapse failure of a structure occurred in Oklahoma City, U.S.A., on April 19, 1995. The Alfred P. Murrah Federal Building was destroyed by the explosion of a truck bomb that knocked out three columns at the ground level, which then triggered the progressive collapse of the whole building [FEMA, 1996]. The most significant and well known case of progressive collapse the World Trade Center in New York City, U.S.A. which was struck by jetliners on September 11, 2001 which started a chain of events leading to the final collapse of the two towers [NIST, 2005]. These three examples of progressive collapse of structures, as shown in Figure 2.1, are milestones in the development of guideline recommendations and code and standard requirements to prevent progressive collapse of buildings. 
The cause of progressive collapse phenomena can be due to human-made hazards (blast or explosion, vehicle impact, fire, etc.) or natural hazards such as earthquakes. Earthquakes can induce strong cyclic lateral forces and severe stress reversals in lateral load resisting systems of structures. These load effects can overload structural members, which can result in the loss of one or more loadcarrying members (especially vertical load-carrying components), that may then lead to failure of additional structural members in other parts of the system and subsequent unzipping effect of progressive collapse of the entire system. Observations of earthquake damage in past earthquakes show that seismic loads can cause structural damage that result in loss of supports in the structure [Moehle et al., 2002; Gurley, 2008]. The initial failure of individual structural elements or components of structural elements can propagate to other adjacent load resisting members in a variety of ways [Starossek, 2007].

\subsection{Structural Resistance to Progressive Collapse}

In order to withstand abnormal loading that can cause progressive collapse, there are several characteristics in the structural design and layout of a structure that can have significant influence on its collapse resistance. These structural characteristics are summarized as follows: 
1. Robustness is the structural ability to survive the event of local failure. A robust structure can withstand the loading so it will not cause any disproportionate damage.

2. Integrity is the condition where the structural members remain connected together even after the presence of the abnormal events. In other words, the structural system will not become separated even after the imposed load has exceeded the design value.

3. Continuity is the interconnection of structural elements in a structural system. In design codes and standards of reinforced concrete structures, continuity is also referred to as continuous steel reinforcement detailing.

4. Ductility is the structural ability to sustain additional deformation after initial failure or yield condition while maintaining the same load carrying capacity in the structural member or system.

5. Redundancy is the capability of other structural members to carry extra load in case some members fail or collapse. This implies that if there is a failure in one of the elements, other elements and the remaining structural system as a whole can still withstand the load.

The structural resistance to progressive collapse phenomenon is the combined effect from all the contributing structural characteristics mentioned above. If a structure has these characteristic conditions, it may be considered to be less 
vulnerable to progressive collapse. Therefore, in designing a structure against progressive collapse, one must consider the comprehensive aspects of the aforementioned conditions.

A structure designed with due consideration of its lateral load resistance against earthquakes in active seismic regions has many similar structural layout and detailing characteristics as those designed to resist progressive collapse due to other load effects. Research has shown that good detailing and strengthening to enhance seismic resistance of a structure can provide a higher safety level against progressive collapse events [Corley, 2002; Hayes et al., 2005].

\subsection{Progressive Collapse Design of Buildings}

Since the early development of structural design against progressive collapse, there have been many improvements in the provisions in codes and standards to provide guidance, design requirements and more realistic and specific guidelines or requirements for the prevention of progressive collapse in structures. Generally, there are two kinds of protection against progressive collapse: intrinsic and extrinsic protections [El-Hacha and Bullock, 2006]. Extrinsic protection is protecting the surrounding location of the building, which is more related to blast loads, and intrinsic protection is protecting the structure itself. Herein, a brief overview of current progressive collapse resistant design provisions and guidelines related to intrinsic protection of building structures are presented. 
The National Building Code of Canada 2005 (NBCC 2005) [NRCC, 2005] and the American Concrete Institute's Building Code Requirements for Structural Concrete 2008 (ACI 318-08) [ACI, 2008] rely on structural integrity requirements to prevent progressive collapse of structures. These requirements are based on the assumption that improving redundancy and ductility by good detailing in reinforcements can help to localize the damage so that it will not propagate to other members, and thus the overall stability of the structure can still be maintained.

The American Society of Civil Engineers' Minimum Design Loads for Buildings and Other Structures 2005 (ASCE/SEI 7-05) [ASCE, 2006] and the National Institute of Standards and Technology's Best Practices for Reducing the Potential of Progressive Collapse in Buildings [NIST, 2007] specifies two alternative design approaches for increasing resistance against progressive collapse: direct design and indirect design. In the direct design approach, resistance to progressive collapse is considered explicitly during the design process by either the alternative load path method or the specific local resistance method. The alternative load path method allows local failure to occur, but the progressive collapse mechanism is averted or bridged over through alternate load paths that distribute the load from the missing member to other redundant members so that the effect of the damage can be absorbed. The specific local resistance method does not allow local failure to occur as it provides sufficient strength on the "key" element to prevent failure of a structural member, which is thus thread-specific. 
While the direct design approach offers a more explicit design solution, the indirect design approach considers resistance to progressive collapse implicitly during the design process through the provisions of minimum levels of strength, continuity, and ductility. ASCE/SEI 7-05 [ASCE, 2006] also states that structures can be designed to sustain or minimize the occurrence of progressive collapse by limiting the effects of a local collapse from spreading to other members.

In the General Services Administration Guidelines [GSA, 2003], it is stated that redundancy, detailing to provide structural integrity and ductility, and capacity for resisting load reversal need to be considered in the design process to make the structure more robust and thus enhance its resistance against progressive collapse. It stipulates an analysis procedure of removing vertical load bearing elements to assess the potential of progressive collapse to occur in a structure. The guidelines also give requirement on maximum allowable collapse area that can occur if one vertical member is lost or collapses. Figure 2.2 shows examples of the maximum allowable collapse area if an exterior or interior column fails. In addition, this document also specifies a demand to capacity ratio limit values for various types of structures to be used in static linear analysis. Menchel et al. [2009] state in their study that some of these values may lead to design with insufficient strength in the structure.

The Unified Facilities Criteria (UFC) 4-023-03 [DoD, 2009] provide details for structural design against progressive collapse. The three design/analysis approaches mention here are the Alternate Path (AP), the Tie Forces (TF), and the Enhanced Local Resistance (ELR). The AP approach is similar to the one stated in 
the ASCE/SEI 7-05 [ASCE, 2006] described previously. The TF approach makes use of the tie forces as shown in Figure 2.3, which are due to catenary actions that enhance continuity, ductility, and redundancy of the structure by keeping the structure together after initial failure of individual structural elements or components. The ELR approach provides flexural and shear resistance for perimeter columns or load bearing walls.

This document specifies four different categories for the design of building structures based on the occupancy and importance as follows:

1. Category I is structures that represent low hazard to human life in case of failure or low occupancy structures including but not limited to agricultural facilities, certain temporary facilities, and minor storage facilities. Structures in this category do not require progressive collapse design.

2. Category II is structures that are not listed in Categories I, III, and IV including but not limited to buildings inhabited by less than 50 people, primary gathering buildings, and high occupancy family housings. Structures in this category can use either the TF for the entire structure and the ELR for the corner and penultimate columns or walls at the first story or the AP for specified column or wall removal locations. 
3. Category III is structures that represent a substantial hazard to human life or significant economic loss in case of failure including but not limited to schools, jails, and health care facilities without surgery or emergency treatment facilities. Structures in this category should use the AP for specified column or wall removal locations and the ELR for the perimeter columns or walls at the first story.

4. Category IV is structures designed as essential facilities and national strategic military assets including but not limited to hospitals, emergency shelters, and power generating stations. Structures in this category should use the TF for the entire structure, the AP for specified column or wall removal locations and the ELR for all perimeter columns or walls at the first and stories.

If adequate tie forces cannot be developed in the vertical structural element, then the AP approach is applied to verify whether the structure can sustain the catenary forces or not, which can be affected by the uniform extension detailing of rebars in reinforced concrete structures [Yi et al., 2008]. Structures with high occupancy or importance required to follow both the AP approach and the TF approach in order to verify not only the catenary resistance but also satisfactory flexural resistance capacity of the design. The horizontal tie forces are prohibited to be concentrated in the beams, girders, and spandrels. They should be carried in the floor or roof so that the floor and roof systems can contribute in the transfer of the vertical loads via 
catenary or membrane action to other redundant horizontal members and finally to the vertical elements of the structure [Stevens et al., 2008].

\subsection{Analysis Methods for Progressive Collapse}

A progressive collapse analysis is needed to determine the capacity of a structure to resist abnormal loadings. In general, there are several methods that can be used: linear static, nonlinear static, linear dynamic, and nonlinear dynamic. Each of them has some advantages and disadvantages from progressive collapse analysis aspects [Marjanishvili, 2004]. A brief summary of different analysis methods is presented herein:

1. Linear static analysis is the fastest and easiest to perform, but it considers neither dynamic effect nor nonlinear effects that result from material and geometric nonlinearity. Also, this analysis is only applicable to the analysis of structures with displacement and strain level below the elastic yield limit.

2. Nonlinear static analysis takes into account the effects of material and geometric nonlinearity, but does not consider the dynamic effect directly in the analysis. The procedure is relatively simple and may provide useful or information about the behaviour of a structure. 
3. Linear dynamic analysis includes the dynamic behaviour of the structural response, but it does not consider the effects of material and geometric nonlinearity. This analysis may not give good results if the structure exhibits large plastic deformations.

4. Nonlinear dynamic analysis provides the most comprehensive and detailed results and includes both material and geometric nonlinearity and dynamic effects. Comparing to the other types of analysis, nonlinear dynamic analysis is the most time consuming.

When a structure undergoes progressive collapse, the response of the structure is affected by dynamic effects [Pretlove et al., 1991; Kaewkulchai and Williamson, 2002]. This requires the dynamic behaviour of a structure to be taken into account in the progressive collapse analysis. It is also expected that nonlinear structural behaviour can significantly affect the progressive collapse behaviour of a structure since before reaching the collapse condition a structure and its member components must have exceeded its elastic limits. Considering these two observations, it can be concluded that the nonlinear static analysis and nonlinear dynamic analysis are the two most appropriate methods for evaluation of progressive collapse behaviour of structures, among the available analysis methodologies.

In nonlinear static analysis, dynamic effects in the responses are not considered directly. Despite this limitation, experiences have shown that the results obtained by nonlinear static analysis can still provide valuable insights on the behaviour of the analyzed structure and the results tend to be conservative in most 
cases. The attractiveness of this method is its simplicity compared to nonlinear dynamic analysis approach. Studies have shown that nonlinear static analysis methods can give good approximations of deformation demands, identify the strength discontinuities, and assess global stability of structural systems [Krawinkler and Seneviratna, 1998]. Nonlinear static analysis has also proven to give good estimates to seismic demands of structures. Therefore, nonlinear static analysis procedure is a valuable alternative method to the more rigorous nonlinear dynamic method for analysis of progressive collapse behaviour of structures. Using the nonlinear static analysis procedure, a capacity curve of a structure can be generated by pushover analysis. A capacity curve provides insight into whether a structure has adequate capacity to resist the loading condition or not. During progressive collapse, dynamic properties of a structure change after failure of one or more members in the system. Therefore to capture the progression of the collapse mechanism, it may require multiple pushover analyses if the analysis tool employed in the simulation does not specially model and capture the progressive changes in structural properties and behaviour of the system.

For seismic progressive collapse evaluation, the analysis procedure should take into account the effects of lateral seismic forces in conjunction with those from gravity loads. It requires an analysis tool that can capture the structural responses from initial localized failure of individual structural elements or components, to partial collapse, collapse and post-collapse behaviour of the structure. Current progressive collapse analysis procedures that only account for gravity load effect may 
not have the capabilities to model and capture the total effects of progressive collapse of structures due to overloading during earthquakes. In addition, falling debris from collapsed members may result in significant impact loading to other members in the remaining system, which also needs to be considered in the analysis.

\subsection{Earthquake Loads and Progressive Collapse}

Seible et al. [2008] have noted that earthquake loads can cause progressive collapse of bridges that is influenced by characteristics of the earthquake such as magnitude, intensity, source mechanism, and attenuation. In dynamic responses of structures during major earthquakes, continuous vibration after initial failure and repeated stress reversals from cyclic inelastic actions can lead to significant deterioration in stiffness, strength, and ductility capacity of the structural system due to accumulated damage effects. This deterioration in turn can affect the progression of failure and ultimate collapse behaviour of the structure. A high intensity earthquake can cause severe failure to the structure. The failure is caused by the increase in the lateral forces demand that results in the development of plastic hinges in regions that were not designed for sufficient ductility [Priestley et al., 1996]. Gravity load can also increase the plastic drift caused by earthquake load that may lead to instability and thus collapse of the structure [Jennings and Husid, 1968]. Seismic progressive collapse is defined as the result of a sequence of events starting from initiation of failure of a single component, due to overstress beyond the elastic 
limit, to degradation of material and member properties (related to stiffness and strength) that are the result of accumulation of damage effects from cyclic stress reversals until the development of collapse mechanisms.

There are a number of experiments that have been carried out to relate collapse caused by axial and shear failures on reinforced concrete columns during earthquakes. A brief summary of some research are presented herein. After evaluating columns that experienced shear failures prior and after flexural yielding, Nakamura and Yoshimura [2002] concluded that axial failure can occur when the shear resistance is reduced to approximately zero. Sezen [2000] also did experiments on columns under cyclic lateral loads and axial loads. The results show that the loss of lateral load capacity is not always followed by the loss of axial load capacity. Elwood and Moehle [2003] added that the shear failure in columns does not necessarily cause the collapse of the whole system. However, shear failure may reduce the axial capacity of the column. Furthermore, columns with lower axial loads tend to have axial failure at relatively large drifts and in contrast, columns with higher axial loads tend to have axial failure at smaller drift ratio and immediately after loss of lateral load capacity.

Ibarra and Krawinkler [2005] explained procedures to assess side-sway collapse on frame structures. This study used deteriorating hysteretic models to compute the collapse capacity of the structures and the results could be used to develop a collapse fragility curve for the structure. However, the assumptions used in the study are more suitable for buildings where the expected failure is, according to 
their terminology, more incremental collapse rather than progressive (or global) collapse. Incremental collapse occurs when the story displacement is excessively large and the second-order $(\mathrm{P}-\Delta)$ effects have significant offset to the first-order story shear resistance [Ibarra and Krawinkler, 2005]. Thus, this method is not very applicable for progressive collapse analysis of bridge structures.

Previous studies on progressive collapse of structures have also provided insights on the effect of seismic strengthening in increasing the capacity of structures to resist progressive collapse due to blast loads. These studies have shown that earthquake detailing and strengthening can improve progressive collapse resistance of buildings against blast load effects [Corley, 2002; Hayes et al., 2005; Ioani et al., 2007]. Gurley [2008] also commented that enhancing seismic ductility of a structure through ductile detailing can help to better develop capacity of the structure to resist progressive collapse mechanism. Despite these findings, there is still a lack of information on progressive collapse behaviour and the collapse mechanism that can be used for improving seismic design of new bridges, and developing more effective strengthening and retrofit methodologies for old bridge stock.

\subsection{Progressive Collapse of Bridges}

Progressive collapse of bridges can be caused by wind, vehicle (ship) impact, earthquakes, or other abnormal loadings. Progressive collapse of bridges (particularly reinforced concrete bridges) have been observed in past major earthquakes such as 
the 1994 Northridge [Mitchell et al., 1995], the 1995 Kobe [Anderson et al., 1996; Kawashima, 2000], the 1999 Chi-Chi [Wallace et al., 2001; Hsu and $\mathrm{Fu}, 2004$ ], and the 2008 Wenchuan [Han et al., 2009] earthquakes, to name a few. Most of the damages occurred on the bridges' piers due to shear failure or combination of shear, flexure, and compression failures. A study by Johnson [2006] states that the large displacement of the pier can cause it to fail and then the failure progresses to the other piers. Several bridges also suffered deck collapse due to improper seating. Some examples of bridge failures during earthquakes can be seen in Figure 2.4. Studies of bridges damaged by earthquake in past major earthquakes have shown that better methodology for earthquake resistant design of new bridges should be developed. Moreover, effective retrofit and strengthening strategies should be devised to enhance the performance and safety of existing deficient bridges if progression of damage from initial failure to ultimate collapse, and its impact on the failure mechanisms of structures, is better understood.

\subsubsection{Progressive Collapse Analysis of Bridges}

The current guidelines and design standard provisions do not include procedures specifically related to progressive collapse analysis of bridges. Although valuable information and insights can be obtained by following the analysis methodologies used for buildings, it is important to recognize that the behaviour of bridges, especially long-span bridges, are different to those of buildings. Bridges have 
some distinctive features, especially during earthquakes, which require special consideration such as:

1. Unequal distribution of horizontal stiffness along the axis of the structure due to unequal height of the piers. A stiffer pier tends to suffer more damage since it attracts higher seismic loads. This in turn can result in a highly unbalanced distribution of damage in the structure. Failure of shorter and stiffer piers can also cause changes to the vibration periods of the bridge making them longer and also having larger displacement responses.

2. Adjoining connected parts of a bridge may affect the bridge properties. During earthquakes, adjacent connected structures such as on- and off-ramps can change the dynamic properties of the bridge as the separation or contact conditions change due to dynamic responses in earthquakes.

3. Different types of soil condition under different bridge pier foundations. The multi-support excitation problem has a greater impact on the structural behaviour and performance in cases of long-span bridges. A different soil support condition under a bridge pier support can amplify or modify the phase, frequency, and intensity characteristics of the input motion to the bridge structure. 
4. Plan irregularity of bridges. The dynamic responses of bridges that are irregular in plan, such as curved bridges are three-dimensional. This can happen even in the simple case of unidirectional ground excitation.

5. Bridges crossing fault rupture zone. In the case of a bridge crosses an active earthquake fault, site-specific seismological study and more rigorous analysis are needed. This problem also needs extra attention to be evaluated in more details.

Nonlinear time history analysis is considered to be the most accurate method of analysis for assessing the structural behaviour during earthquakes including progressive collapse. Bridges have a number of structural features that may affect their inelastic behaviour compared to buildings such as expansion joints, bearings, and drop in spans, to name a few, in their structural layout.

\subsubsection{Progressive Collapse Resistant Design of Bridges}

Design of bridges against progressive collapse is needed not only to prevent catastrophic failure or total collapse of new bridges against severe effects of major earthquakes, but also to obtain crucial information and insights for devising effective strengthening and upgrade strategies for existing old and deficient bridge stock. The design philosophy adopted for progressive collapse resistant design of buildings can be applied to design of bridges. The methods described previously in Section 2.3 can 
be adapted for design or retrofit of bridges. For the design of long-span bridges, the design requirement of progressive collapse prevention may be accommodated through interruption of the collapse mechanism. A good example is the Confederation Bridge which crosses the Northumberland Strait between Prince Edward Island and New Brunswick, Canada. It has a total length of $12.9 \mathrm{~km}$. The bridge is designed such that failure of a single span will not lead to failure of other spans [Ghali and Tadros, 1997, 1998; Starossek and Sauvageot, 1998]. In other words, the whole system is prevented to experience collapse by limiting or isolating the local failure from spreading to or from other parts of the structure as shown in Figure 2.5.

Starossek [2006] also noted that there are three shortcomings on the current design codes and standards, which are based on the reliability theory:

1. The design code only considers the local failure, not global failure. Consequently, the structural safety is controlled at the local level, whereas the condition at the global level is not guaranteed. Different structures will have different responses to local failure and the assumption that the global system is safe when the local elements are safe is not always true.

2. The design code does not take into account low probability events and unforeseeable incidents. The simplifications used to develop the codes do not consider small events that can possibly cause collapse of a structure. However, if the number of elements in a structure is 
significantly large, a very low probability event can amplify the probability of global failure.

3. The design code has the fundamental probabilistic concept that requires specific admissible probability of failure. Since the cause of failure that leads to progressive collapse is uncertain, the probability of such failure is also hard to be defined. Hence, it is difficult to formulate the admissible probability of failure of such event.

Starossek [2006] also argued that although the current design procedure can be implemented, additional assessments are still needed. These additional assessments, which are deterministic analyses, should focus more on the objectives and should be practical in application.

\subsection{Analytical Tools}

There are several factors that must be considered in a progressive collapse analysis to determine the capability of a structure in resisting abnormal loadings. Some researchers have developed their own software platforms to include progressive collapse algorithms into the program. Many commercial structural analysis software packages are also available that can be utilized for this purpose and some even have specific options for progressive collapse of structures. Some of these analytical tools are discussed in this section. 


\subsubsection{Finite Element Method-Based Tools}

Researchers have used general finite element software packages for frame structures such as SAP 2000, STAAD Pro, PERFORM 3D, and OpenSees to assess the progressive collapse behaviour of structures [Grierson et al., 2005; Marjanishvili and Agnew, 2005; Powell, 2005; Bao et al., 2008; Kim et al., 2009]. The finite element analysis software packages for continuum systems, such as FLEX, ANSYS, ABAQUS, LARSA, and DIANA, have also been used and compared with each other [Bao et al., 2008; Smilowitz, 2002]. Furthermore, Miao et al. [2007] have developed computer software called THUFIBER using a fibre model for modelling of nonlinear behaviour of structural elements that can be used for progressive collapse analysis. These analysis tools typically assume the analyzed structures remain continuous, meaning that even if collapse occurs the structure still maintains its continuity. The collapse mechanism is represented through the behaviour of plastic hinges formed due to flexural overstress in members. Using these analysis tools, the effects of member separation due to fracture failure can be approximated by removing specific failed individual members from the analysis model to assess the capability of other members to withstand progressive collapse.

The first software capable of progressive collapse analysis was developed by Gross and McGuire [1983]. This software allows the user to selectively remove any member in the structure to determine the consequence of damage, so as to evaluate if 
a collapse may occur. The structure is modelled by $2 \mathrm{D}$ frame elements and the debris loading is modelled as several distributed point loads along the member. This may not be completely realistic, but it can be used to simplify the case in finite element modelling. Kaewkulchai and Williamson $[2002,2004]$ have also developed software for progressive collapse analysis of planar frame structures. Their program can take into account the effect of strength and stiffness degradation with a discrete element model.

Another finite element code was developed by Toi and Isobe [1993] to expand the current finite element analysis with the so-called the Adaptively Shifted Integration (ASI) technique. Their analysis method takes into account plastic collapse of framed structures using linear Timoshenko or cubic beam element formulations. The basis of this ASI technique is shifting the numerical integration points for the calculation of stiffness matrices immediately after the occurrence of plastic hinges. A later version of this method has been applied for seismic collapse analysis of framed structures [Isobe and Tsuda, 2003].

The FEM can also take into account the stiffness and strength degradation on the failed elements such as techniques suggested by several researchers [Phung, 2005; Ibarra et al., 2005; Phung and Lau, 2006; Moehle et al., 2006; Elwood and Moehle, 2008]. Numerical approach of using Lagrangian formulation [Sivaselvan and Reinhorn, 2006] could also be adopted as an option in the FEM. Lu et al. [2008] introduced a technique to deactivate the element after it reaches a specified limit using fibre-based model. For bridges, Banerjee and Shinozuka [2004] proposed drift 
limit and ductility demand criteria to assess whether a part of a bridge has experienced collapse. However, these techniques still consider continuous dynamic response without any separation of elements and collision from the debris. Developments of the FEM techniques that take into account element separation and/or contact have been reported by several researchers [Isobe and Tsuda, 2003; Talaat and Mosalam, 2008; Mosalam et al., 2008], but there are still some complications in the implementation of the proposed techniques. The complications are mainly caused by the tedious procedures once an element fails and starts to separate or re-contact.

The FEM has also been used for decades as a tool to model crack propagation in material using fracture mechanics theories [Hillerborg et al., 1976]. Meshless FEM techniques are also commonly used for crack analysis [Belytschko et al., 1995]. A more recent developments of the Extended Finite Element Method (XFEM) procedures for crack analysis also show that crack propagation can be followed with a good accuracy [Sukumar et al., 2000; Moës and Belytschko, 2002; Areias and Belytschko, 2005]. However, the application of these methods to date is only on uniform material with small-scale problems. The applications for nonuniform material (e.g., reinforced concrete) and large-scale problems such as building or bridge structures have not been investigated and most probably will be more time consuming. 


\subsubsection{Non-Finite Element Method-Based Tools}

Researchers in the field of rock mechanics have used the Discrete (or Distinct) Element Method (DEM) [Cundall, 1971] to analyze and simulate collapse of granular materials. For analysis of concrete structures, a modification of the DEM called the Extended Discrete Element Method (EDEM) was proposed by Meguro and Hakuno [1989, 1992]. This method has shown good results in simulating typical global collapse behaviours of concrete frames under earthquake loads. However, in the analysis, the reinforcements are not directly modelled and it is also time consuming [Hakuno and Meguro, 1993]. More recently, Sun et al. [2003] has introduced a lattice and truss model to the EDEM to analyze a single-column reinforced concrete pier subjected to an earthquake. However, this model still cannot take into account the inertial effect of falling debris. In addition, the results show that this model gives higher axial strengths than the experiments and improvements are still needed for the simulation accuracy before collapse [Sun et al., 2003].

Some researchers have utilized a hybrid method called the Finite - Discrete Element Method (F/DEM) in progressive collapse analysis of structures which combines the FEM and DEM [Munjiza, 2004; Lu et al., 2009]. This method can simulate the progressive collapse phenomena but the application to calculate the internal forces is not reported. In the F/DEM, the analysis procedures switch from the FEM to the DEM after occurrence of separation of structural members in the system. 
Recently, the Applied Element Method (AEM) [Tagel-Din and Meguro, 1999] has been developed with the capability to model progressive collapse behaviour of structures. The AEM has been implemented in a software package called Extreme Loading for Structures (ELS) in which the structure is modelled by 3D elements interconnected to each other by springs to represent the interface effects, such as stresses, strains, deformations, and fracture failure in the structure. This analysis tool explicitly considers the effects of element separation and discontinuity as well as debris loads caused by collapsed members and the resulting inertia impact load effects. Studies have shown that ELS based on the AEM theory can give good estimations to large displacements and deformations of structures undergoing progressive collapse [Meguro and Tagel-Din, 2002; Wibowo et al., 2009]. In this thesis, the seismic progressive collapse behaviour of reinforced concrete bridges are analyzed using the AEM. 


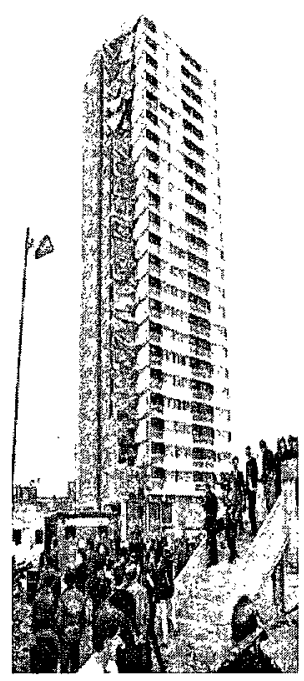

(a)

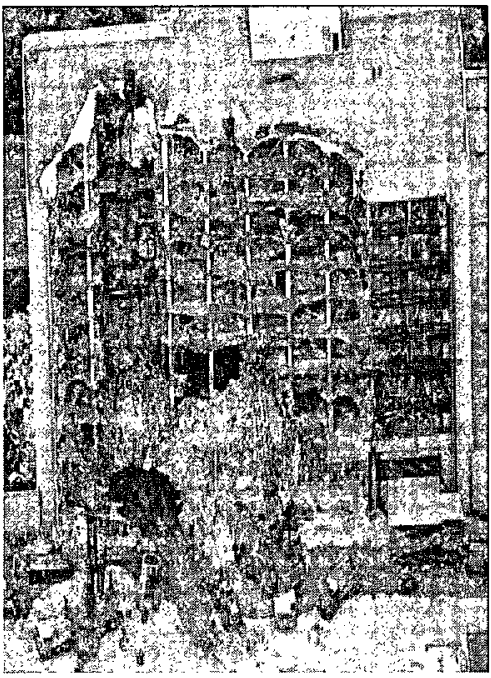

(b)

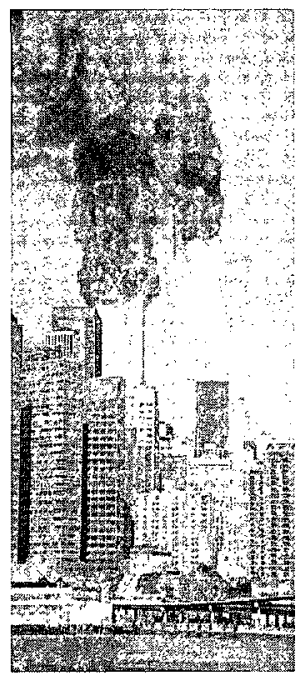

(c)

Figure 2.1. Collapses of (a) Ronan Point Tower, (b) Alfred P. Murrah Building, and (c) World Trade Center

[Figure 2.1(a) is Obtained from Daily Telegraph $\subset$ 1968, Figure 2.1(b) is Obtained from CBS News, and Figure 2.1(c) is Obtained from Jim Collins of the Associated Press] 

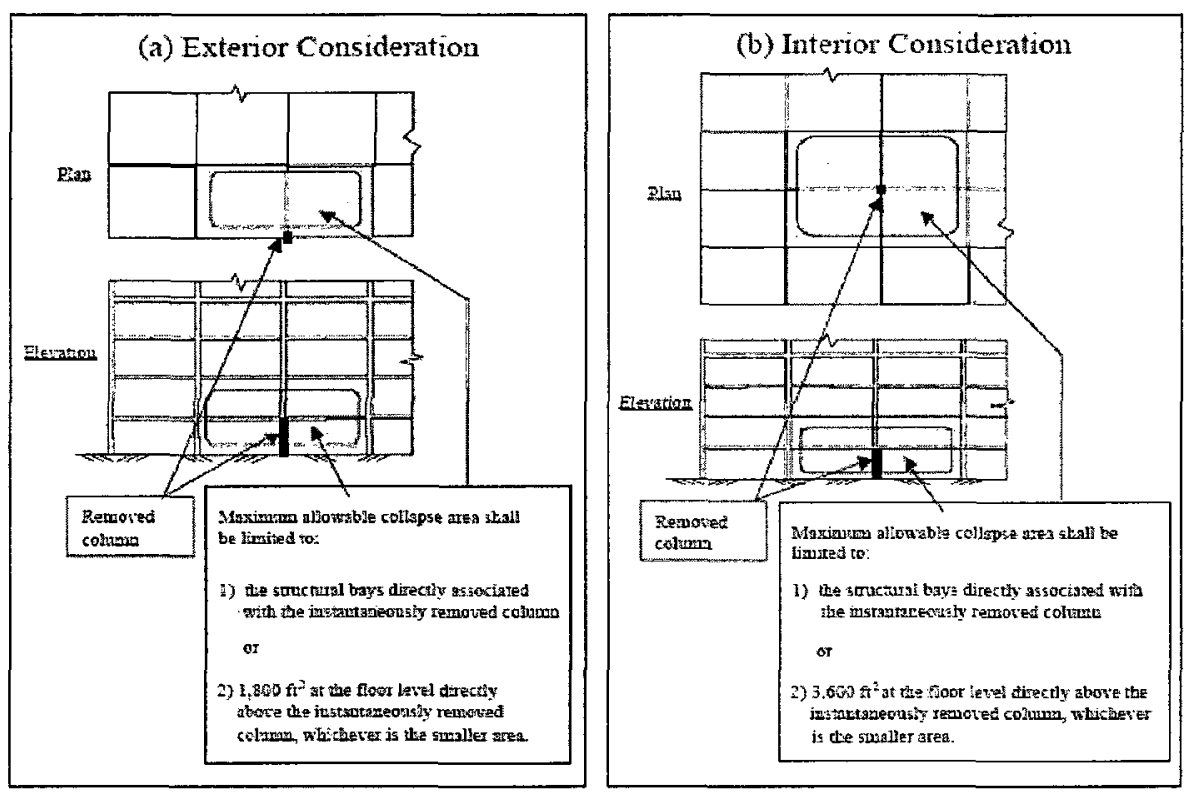

Figure 2.2. Examples of Maximum Allowable Collapse Area in GSA Guidelines [GSA, 2003] 


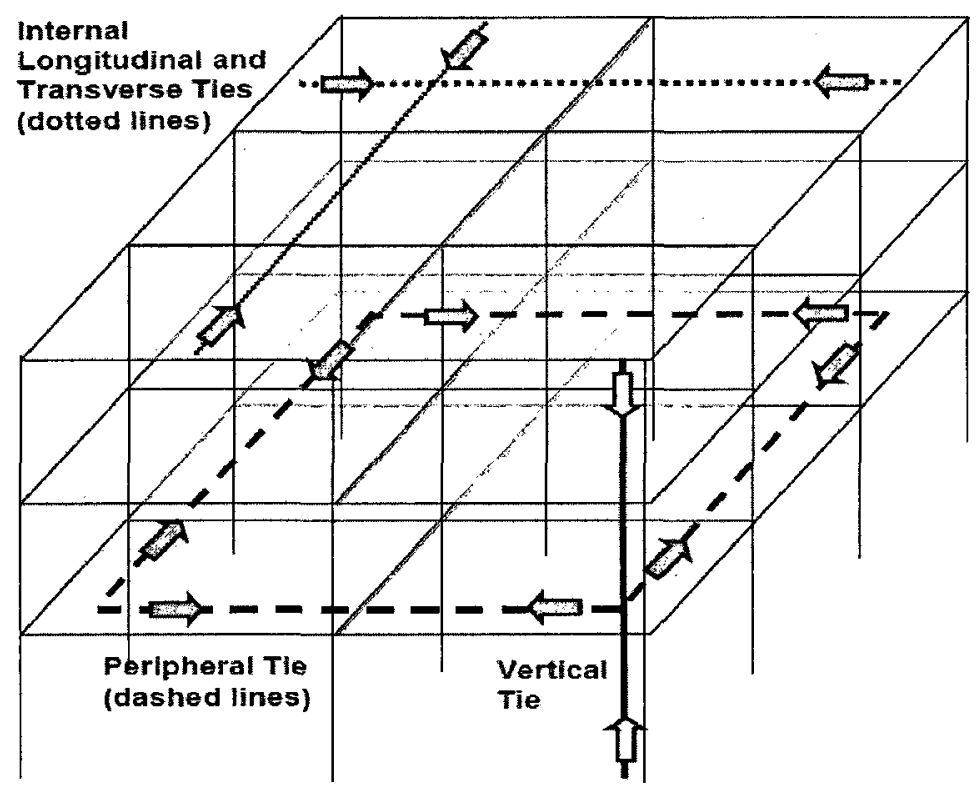

Figure 2.3. Tie Forces Described in UFC 4-023-03 [DoD, 2009] 
(a)
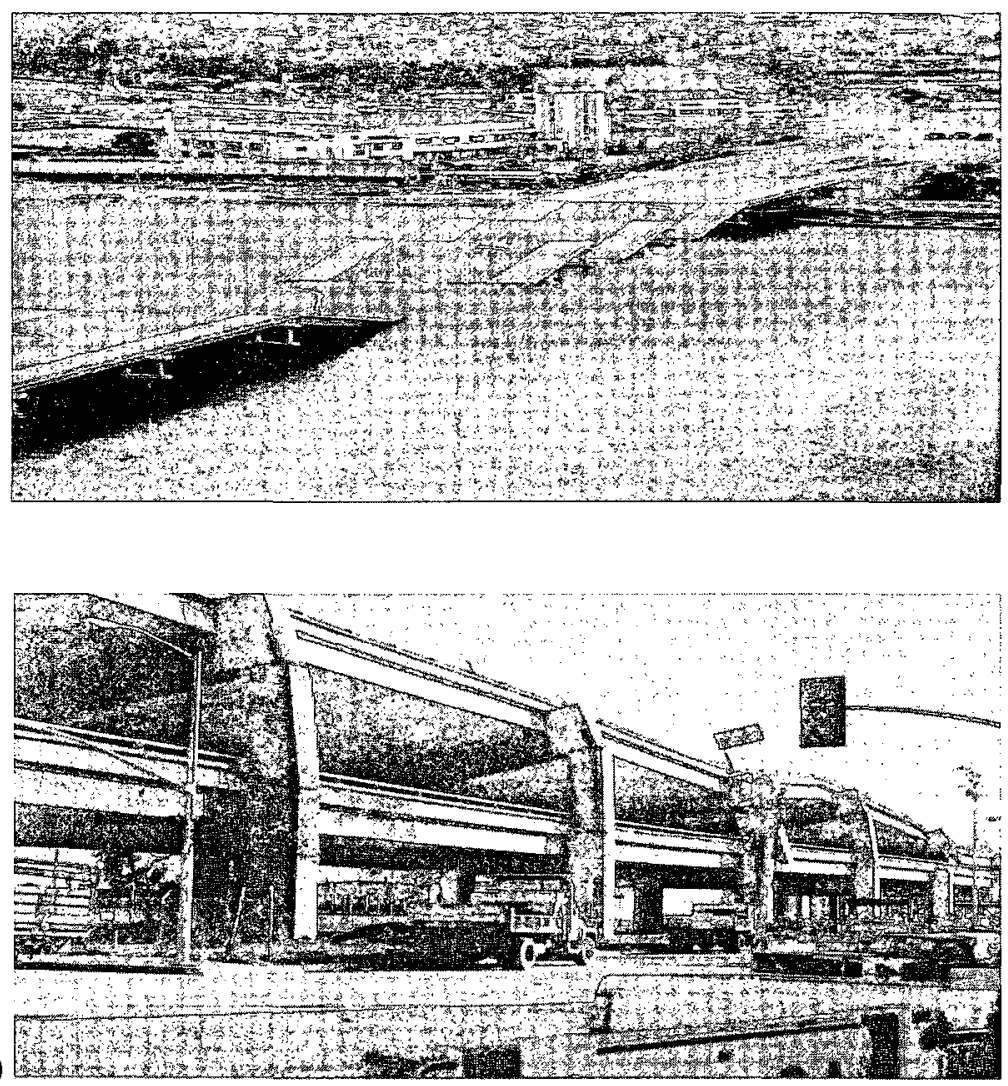

(b)

(c)

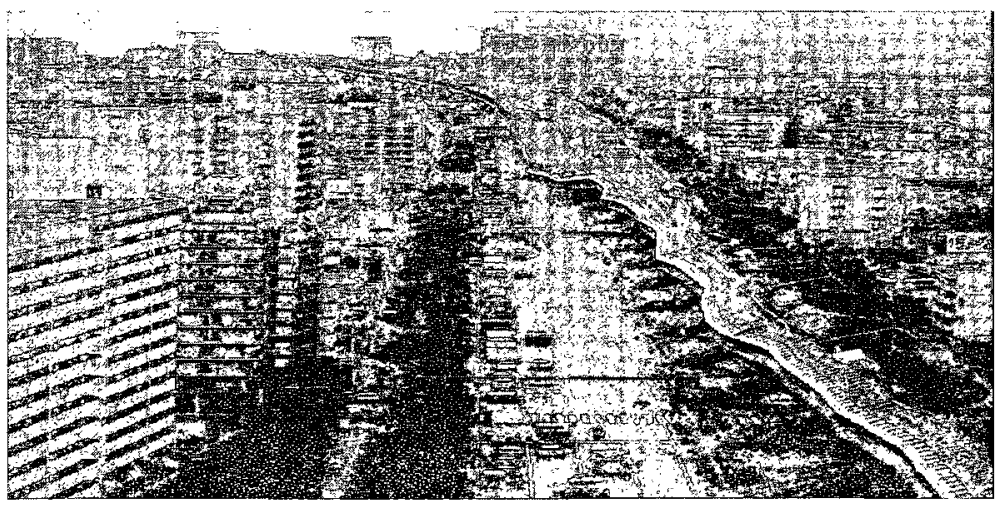

Figure 2.4. Collapse of Bridges during the (a) 1964 Niigata, (b) 1989 Loma Prieta, and (c) $1995 \mathbb{K}$ obe Earthquakes

[Figures 2.4 (a) and (c) are Courtesy of the National Information Service for Earthquake Engineering, Earthquake Engineering Research Center, University of California, Berkeley] 

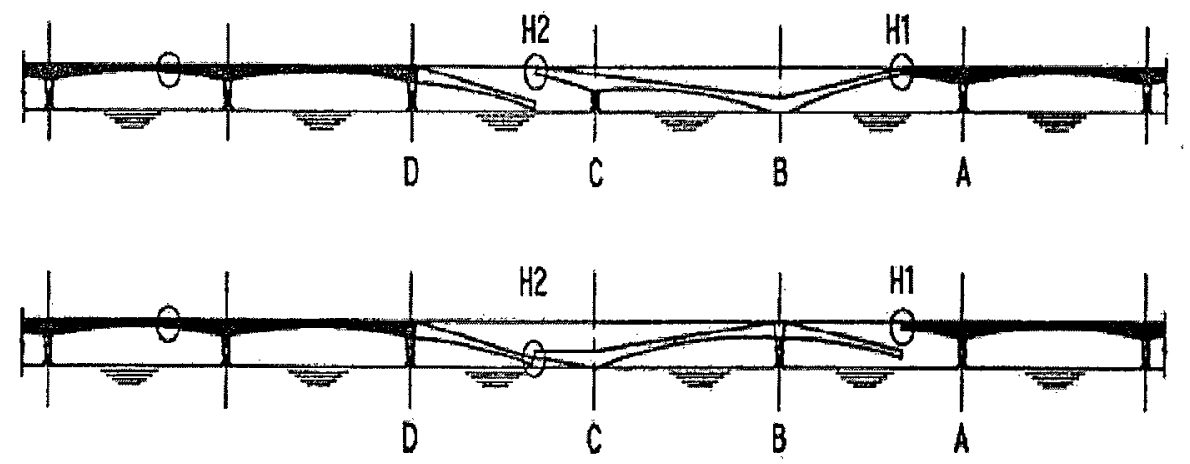

Figure 2.5. Progressive Collapse Resistant Design Applied on the Confederation Bridge [Starossek, 2006] 


\section{CHAPTER 3}

\section{Overview of the Applied Element Method}

In this chapter, the theoretical background of the Applied Element Method is discussed and a general overview of the application of the method in Extreme Loading for Structures software is presented.

\subsection{Theory and Formulation of the Applied Element Method}

In general, the numerical methods for structural analysis can be classified into two categories based on the modelling domain: continuum and discrete modelling domains. The Finite Element Method (FEM) is an example of analysis method of continuum domain assumption. The Discrete Element Method (DEM) [Cundall, 1971], the Extended or Modified Discrete Element Method (EDEM or MDEM) [Meguro and Hakuno, 1989, 1994; Meguro et al., 1991], and the Rigid Body and Spring Model (RBSM) [Kawai, 1980; Kikuchi et al., 1992] are examples of methods based on discrete domain assumption.

Continuum analysis models basically subdivide the analysis domain into small finite elements with properties representative of the domain material. For structural analysis, although continuum models have been proven to be accurate even 
in highly nonlinear problems due to material and geometry nonlinearities, problem starts to occur when the behaviour of structure progresses from a continuum state to a discrete state such as during collapse event.

Discrete analysis models, on the other hand, are based on discrete element modelling techniques. The main advantage of discrete models is the capability of this approach in simulating the cracking process [Kikuchi et al., 1992]. However, the behaviour of crack propagation in discrete analysis models that use rigid elements in the formulation is highly dependent on the element shape, size, and arrangement [Kikuchi et al., 1992; Ueda and Kambayashi, 1993].

To overcome the limitations of continuum and discrete analysis models, a method called the Applied Element Method (AEM) has been proposed by Tagel-Din and Meguro [1999] that combines the advantages from continuum analysis models and discrete analysis models. The analysis application domain of the AEM is presented in Figure 3.1. In brief, the main advantages of the AEM formulations are separation of structural elements can be easily modelled and the inertial impact forces of falling debris are taken into account during the analysis. In dynamic analysis of structures, the effects of geometrical changes in the structure including rigid body motion during failure are accurately modelled [Tagel-Din and Meguro, 2000b]. In progressive collapse analysis, the AEM can model the behaviour of a structure from initiation and propagation of cracks to separation due to fracture failure of structural elements. 


\subsubsection{Element Formulation in Static Small Deformation Range}

In the AEM, a structure is modelled by dividing it into an assembly of small elements as shown in Figure 3.2. Two adjacent elements are assumed to be connected at discrete points along their edges by a pair of normal and shear springs with the stiffness, $K_{n}$ and $K_{s}$, respectively defined by:

$$
K_{n}=\frac{E d t}{a} \quad \text { and } \quad K_{s}=\frac{G d t}{a}
$$

where $d$ is the distance between springs, $t$ is the element thickness, $a$ is the length of the representative area, $E$ is the material Young's modulus, and $G$ is the material shear modulus. Equation 3.1 implies that each spring represents the stiffness of a volume of the modelling material with the dimensions $d, t$, and $a$. In the case that reinforcement bar (rebar) is present, rebar stiffness is added to the material stiffness in Equation (3.1).

In the two-dimensional (2D) model, each element has three degrees-offreedom (DOFs) to represent the element's rigid body motion. The coefficients of the stiffness matrix of the element are determined by applying a unit displacement to one DOF at a time while keeping the remaining DOFs fixed. The contributions of the contact springs shown in Figure 3.3 to the displacement DOFs $u_{1}$ and $u_{2}$ and the rotational DOF $\mathrm{u}_{3}$ are shown in the matrix form as follows: 


$$
\left[\begin{array}{c|c|c}
\sin ^{2}(\theta+\alpha) K_{n} & -K_{n} \sin (\theta+\alpha) \cos (\theta+\alpha) & \cos (\theta+\alpha) K_{s} L \sin (\alpha) \\
+\cos ^{2}(\theta+\alpha) K_{s} & +K_{s} \sin (\theta+\alpha) \cos (\theta+\alpha) & -\sin (\theta+\alpha) K_{n} L \cos (\alpha) \\
\hline-K_{n} \sin (\theta+\alpha) \cos (\theta+\alpha) & \sin ^{2}(\theta+\alpha) K_{n} & \cos (\theta+\alpha) K_{n} L \cos (\alpha) \\
+K_{s} \sin (\theta+\alpha) \cos (\theta+\alpha) & +\cos ^{2}(\theta+\alpha) K_{s} & -\sin (\theta+\alpha) K_{s} L \sin (\alpha) \\
\hline \cos (\theta+\alpha) K_{s} L \sin (\alpha) & \cos (\theta+\alpha) K_{n} L \cos (\alpha) & L^{2} \cos ^{2}(\alpha) K_{n} \\
-\sin (\theta+\alpha) K_{n} L \cos (\alpha) & -\sin (\theta+\alpha) K_{s} L \sin (\alpha) & +L^{2} \sin ^{2}(\alpha) K_{s}
\end{array}\right]
$$

where $L$ is the distance from the location of the contact spring to the centroid of the rigid element, and $\theta$ and $\alpha$ are the orientation angles of the rigid element, as defined in Figure 3.3. The stiffness matrix presented in Equation (3.2) is one-quarter of the element's global stiffness matrix representing the deformation interaction with its surrounding on one of the four sides of the rectangular rigid element. The global stiffness matrix, $\boldsymbol{K}_{G}$, is generated by adding up the contributions of all springs on all sides of the element, similar in concept of generating the global stiffness matrix in the FEM. Accordingly, the developed global stiffness matrix is an average stiffness matrix for the element corresponding to the stress state around the element [Meguro and Tagel-Din, 2000]. The governing equation is written as follows:

$$
\boldsymbol{K}_{\boldsymbol{G}} \boldsymbol{U}=\boldsymbol{F}
$$

where $U$ is the displacement vector and $\boldsymbol{F}$ is the applied load vector. In load controlled analysis case, the applied load vector, $\boldsymbol{F}$, is known before the analysis whereas in displacement controlled analysis case, the load is applied as imposed displacement at one or more DOFs. 
Element rotation is resisted by a set of normal and shear springs. The contribution to rotational stiffness, $K_{r}$, from the normal springs can be calculated as follows [Meguro and Tagel-Din, 2001]:

$$
K_{r}=\int_{z=-b / 2}^{z=b / 2} \frac{E t}{b} z^{2} d z=\frac{E t b^{2}}{12}
$$

where $E$ is the Young's modulus, $t$ is the element thickness, $b$ is the element height, and $z$ is the distance from the spring to the centroid as shown in Figure 3.4. Furthermore, the element rotational stiffness is the addition of all the rotational stiffness calculated separately for each spring that leads to:

$$
K_{r}=\frac{E t b^{2}}{4 n^{3}} \sum_{i=1}^{n}(i-0.5)^{2}
$$

where $i$ is the spring number and $2 n$ is the number of springs.

The AEM formulation has been proven to give good accuracy in the nonlinear analysis of reinforced concrete structures subjected to small deformations [Meguro and Tagel-Din, 1998]. However, in order to be used for large deformation analysis, the geometrical changes in the structure's shape should also be considered. The modelling of the effect of the geometrical changes in the element formulation is discussed in the following sections. 


\subsubsection{Element Formulation in Static Large Deformation Range}

The stiffness matrix utilized in Equation (3.1) is obtained based on the assumption that the structure undergoes small deformations. In order to consider the effects of geometrical changes in a structure in large displacement analysis, there are two factors that need to be considered:

1. The stiffness matrix calculation for the new structural geometry.

2. The internal forces redistribution due to geometrical changes.

In the FEM, these factors are taken into account by the addition of the geometric stiffness matrix. In the formulation of the AEM, the geometric stiffness matrix is not needed which makes the method more general and easier in its implementation without affecting the accuracy of the analysis. In the AEM, the general governing equation of motion under static loading is written as follows:

$$
K \Delta U=\Delta f+R_{M}+R_{G}
$$

where $K$ is the nonlinear stiffness matrix, $\Delta f$ is the incremental applied load vector, and $\Delta \boldsymbol{U}$ is the incremental displacement vector. Two new terms are introduced, $\boldsymbol{R}_{M}$ and $\boldsymbol{R}_{G} \cdot \boldsymbol{R}_{M}$ is the residual force vector due to cracking or incompatibility between strains and stresses at the spring location. $\boldsymbol{R}_{G}$ is the residual force vector due to geometrical changes of the structure during loading. In the AEM formulation, the stiffness matrix of the structure is computed after applying the geometrical changes. 
Material nonlinearity is taken into account during calculation of the matrix $\boldsymbol{K}$ and vector $\boldsymbol{R}_{M}$ [Meguro and Tagel-Din, 1999].

To calculate the residual forces due to the geometrical changes, the following steps are used in the AEM formulation [Meguro and Tagel-Din, 1999]:

1. Assuming that $\boldsymbol{R}_{M}$ and $\boldsymbol{R}_{G}$ are zero, solve the equation to get the incremental displacement vector $\Delta \boldsymbol{U}$ by displacement or load controlled analysis.

2. Modify the structural geometry according to the calculated incremental displacements. This also changes the location of the contact points.

3. Modify the direction of the spring force vectors according to the new element configuration. The geometrical changes cause incompatibility between applied forces and internal stresses.

4. In case of nonlinear material, check the state of cracking and calculate the material residual load vector, $\boldsymbol{R}_{M}$. In elastic analysis, the value of this vector is zero.

5. Calculate the element force vector, $\boldsymbol{F}_{\boldsymbol{M}}$, from surrounding springs of each element.

6. Calculate the geometrical residual vector around each element by 


$$
R_{G}=f-F_{M}
$$

Equation (3.7) implies that the geometrical residuals have taken into account the incompatibility between the external applied forces, $f$, and the internal forces, $\boldsymbol{F}_{\boldsymbol{M}}$, due to the geometrical changes of the structure.

7. Calculate the stiffness matrix of the structure in the new configuration. In case of nonlinear material, also consider the stiffness changes at each spring location due to cracking or yield of rebar.

8. Apply a new load or displacement increment and repeat steps 1 to 7 .

To reduce the calculation time, residuals obtained from the previous increment can be incorporated in the solution of Equation (3.6). However, there are two limitations concerning the formulation:

1. During large deformation (i.e., buckling) analysis, complete symmetry of the structure and loading should be avoided. This can easily be achieved by slightly changing the value of one of material parameters of the structure.

2. Small deformation theory is assumed during each increment which means that small load or displacement increments should be used in 
order the analysis can follow the geometrical changes of the structures.

\subsubsection{Element Formulation in Dynamic Small Deformation Range}

The general governing dynamic equation of motion in small deformation range is expressed as a second order differential equation as follow:

$$
M \Delta \ddot{U}+C \Delta \dot{U}+K \Delta U=\Delta f(t)-M \Delta \ddot{U}_{g}
$$

where $\boldsymbol{M}$ is the mass matrix, $\boldsymbol{C}$ is the damping matrix, $\boldsymbol{K}$ is the nonlinear stiffness matrix, $\Delta f(t)$ is the incremental applied load vector for each time step. $\Delta U, \Delta \dot{U}$, $\Delta \dot{U}$, and $\Delta \ddot{U}_{g}$ are the incremental displacement, velocity, acceleration, and ground acceleration vectors respectively. Equation (3.8) can then be solved iteratively by any step-by-step numerical direct integration method. The Newmark's Beta method is selected here. The procedures of this method can be found in structural dynamics textbooks [Humar, 2002; Clough and Penzien, 2003; Chopra, 2007].

In the formulation here, the element mass and inertia effects are assumed to be lumped at the element's centroid. For example, the mass matrix of square shaped elements is defined by:

$$
\left[\begin{array}{l}
M_{1} \\
M_{2} \\
M_{3}
\end{array}\right]=\left[\begin{array}{c}
d^{2} t \rho \\
d^{2} t \rho \\
d^{4} t \rho / 6
\end{array}\right]
$$


where $d$ is the element length, $t$ is the element thickness, $\rho$ is the density of the element's material. $M_{1}$ and $M_{2}$ are the element mass corresponding to DOFs $\mathrm{u}_{1}$ and $\mathrm{u}_{2}$ respectively and $M_{3}$ is the element moment of inertia around the centroid corresponding to DOF $\mathbf{u}_{3}$. The mass matrix in this case is a diagonal positive definite matrix. The mass matrix plays an important role in the analysis of rigid body motion since inertial forces have significant effects in failure mechanism during collapse of the structure.

The static stiffness matrix becomes singular after fracture failure of an element. This singularity is caused by cracking and separation of elements where the failed element behaves differently from a normal element. The singularity of the stiffness matrix means that the determinant reduces gradually to become zero at fracture. In general, the solution of an ill-conditioned matrix is inaccurate. In other words, the results obtained just before failure or partial failure of the structure may not be accurate, even if the analysis is displacement controlled. This problem is overcome in the dynamic analysis formulation because summation of the inertial effect of the system with the stiffness term in the equation of motion mass matrix to the stiffness matrix results in a positive definite dynamic stiffness matrix even after failure [Tagel-Din and Meguro, 2000b].

Damping in the structure is considered as internal damping and external damping. Internal damping is caused by the behaviour of structural components under 
the applied loadings. In reinforced concrete structures, internal damping can occur because of the following reasons:

1. Cracking of concrete.

2. Energy dissipation during loading and unloading process in compression springs.

3. Unloading of reinforcement after yield.

4. Energy dissipation due to the process of crack closure and opening (when the crack is "closed", shear stiffness is assumed to be equal to the initial stiffness; after reopening of crack, shear forces developed during crack closure are distributed which result in dissipation of shear energy stored during the crack closure).

However, the damping matrix only accounts for external damping, or viscous damping, and the effect becomes dominant in elastic stage. The damping matrix is assumed proportional to the mass matrix and defined as:

$$
\boldsymbol{C}=2 \xi \omega_{1} \boldsymbol{M}
$$

where $\xi$ is the damping ratio and $\omega_{1}$ is the first natural frequency of the structure. The values of the natural frequencies are determined by the eigenvalue analysis described in Section 3.1.5. 


\subsubsection{Element Formulation in Dynamic Large Deformation Range}

Consideration of large deformation in dynamic analysis generally follows the same concept as in static case previously explained in Section 3.1.2. The main idea is to expand the equation of motion from the small deformation range to include the effects of large deformation. In the AEM formulation, the general dynamic equation of motion in large deformation range is expressed as:

$$
M \Delta \ddot{U}+C \Delta \dot{U}+K \Delta U=\Delta f(t)+R_{M}+R_{G}
$$

where $\boldsymbol{M}$ is the mass matrix, $\boldsymbol{C}$ is the damping matrix, $\boldsymbol{K}$ is the nonlinear stiffness matrix, and $\Delta f(t)$ is the incremental applied load vector for a time step. $\Delta \boldsymbol{U}, \Delta \dot{U}$, and $\Delta \ddot{U}$ are the incremental displacement, velocity, and acceleration vectors respectively. $\boldsymbol{R}_{M}$ is the additional load vector due to the nonlinear behaviour of the material and $\boldsymbol{R}_{\boldsymbol{G}}$ is the additional load vector due to the geometrical changes as explained previously. Therefore, Equation (3.11) represents the equilibrium equation between the applied external forces and the internal forces.

The steps to obtain the residual forces due to the geometrical changes in the dynamic analysis are slightly different to those in static analysis. The formulation of the residual force vector in the dynamic analysis is as follows [Tagel-Din and Meguro, 2000b]:

1. Obtain the internal forces caused by the gravity loads under static condition. 
2. Apply a load or displacement increment, $\Delta f(t)$.

3. Solve Equation (3.11) using Newmark's Beta method assuming $\boldsymbol{R}_{M}$ and $\boldsymbol{R}_{G}$ are zero to get the incremental displacement.

4. Calculate the incremental strains and stresses.

5. Calculate the incremental and total velocities and accelerations.

6. Modify the structural geometry according to the calculated incremental displacements by updating the locations of elements.

7. Modify the spring force vectors directions according to the new element configuration. Changing the direction of spring forces surrounding the elements leads to incompatibility between applied loads and internal stresses, also damping forces and inertia forces.

8. Check the state of stress and cracking from the calculated stresses and calculate the material residual load vector $\boldsymbol{R}_{M}$.

9. Calculate the element force vector, $\boldsymbol{F}_{\boldsymbol{M}}$, from surrounding springs of each element.

10. Calculate the geometrical residual vector around each element by:

$$
R_{G}=f(t)-M \ddot{U}-C \dot{U}-F_{M}
$$


Equation (3.12) implies that the geometrical residuals have taken into account the incompatibility between the external applied internal forces, also the damping and inertia forces due to the geometrical changes during analysis.

11. Update the stiffness matrix from the new configuration and considering state of stress and cracking.

12. Apply a new load or displacement increment and repeat steps 1 to 11.

The technique to reduce the calculation time by incorporating the residuals obtained from the previous increment in the solution of Equation (3.12) can also be applied.

There are several assumptions in the element formulations [Tagel-Din and Meguro, 2000b]:

1. The residual forces are calculated based on the total stress values.

2. Gravity loads are considered as external applied forces.

3. Small deformation theory is assumed during each increment, which means time increment should be small.

The formulation discussed above is also applied in static analysis by taking the mass and damping matrices to be zero. However, there is a major limitation in the case of static analysis which is separation of elements during analysis is not allowed since it makes the stiffness matrix singular. In other words, in static analysis the 
continuity between elements should be maintained and hence the behaviour after separation cannot be observed.

\subsubsection{Eigenvalue Analysis}

Eigenvalue analysis is carried out to evaluate the vibration properties of natural vibration modes and natural frequencies or periods of a structure. The results of eigenvalue analysis of eigen-values give the vibration frequencies and eigenvectors are the vibration modes. The general equation for eigenvalue problem of a structure experiencing undamped free vibration is:

$$
\left[\omega^{2} \boldsymbol{M}-\boldsymbol{K}\right] \boldsymbol{U}=0
$$

where $\boldsymbol{M}$ is the mass matrix, $\boldsymbol{K}$ is the stiffness matrix, $\boldsymbol{U}$ is the displacement vector, and $\omega$ is the eigenvalue vector. There are many techniques to solve eigenvalue problems. However, in the formulation here, the method of vector iteration with shifts is adopted. This technique is chosen since it provides a practical tool for computing as many pairs of natural vibration frequencies and modes of a structure as desired.

\subsubsection{Material Modelling}

In reinforced concrete member, the concrete and steel rebar are represented by distinct springs, namely concrete spring and reinforcement spring. Reinforcement 
springs are located on the surface of the element at the exact location where the rebars are placed.

To model the concrete under axial or normal stresses in the AEM, Maekawa compression model [Okamura and Maekawa, 1991] is adopted. This model is shown in Figure 3.5. In this model, the initial Young's modulus, the fracture parameter, and the compressive plastic strain are used to define the envelope for normal stress and strain. Therefore, it allows unloading and reloading to be conveniently described. The tangent modulus is calculated based on the strain at the spring location. Biaxial confinement effects in compression zones are considered by adopting the Kupfer biaxial failure function [Kupfer et al., 1969]. A modified compressive strength, $\mathrm{f}_{\mathrm{c}, \mathrm{eq}}$, is calculated as follows:

$$
\mathrm{f}_{\mathrm{c}, \mathrm{eq}}=\frac{1+3.65\left(\sigma_{1} / \sigma_{2}\right)}{\left(1+\sigma_{1} / \sigma_{2}\right)^{2}} \mathrm{f}_{\mathrm{c}}
$$

where $\sigma_{1}$ is the normal stress, $\sigma_{2}$ is the secondary stress, and $\mathrm{f}_{\mathrm{c}}$ is the compressive strength from the stress-strain relationship. Further discussion on $\sigma_{1}$ and $\sigma_{2}$ is given in Section 3.1.7. Equation (3.14) indicates that the compressive resistance associated with each spring is a variable and depends mainly on the stress state at the spring location.

After peak stress, the spring stiffness is assumed to have a minimum value to avoid negative stiffness. In other words, the Maekawa compression model is adopted until $1 \%$ of concrete strain in compression then the minimum stiffness value $(0.01$ of 
the initial value) is assumed so the connection of the element to the other element is maintained. This results in a difference between the calculated stress and the stress corresponding to the spring strain. These residual stresses are then redistributed during the next loading step by applying the equivalent redistributed force values in the opposite direction. For concrete springs in tension, the spring stiffness is assumed to be the initial stiffness until the cracking point is reached. After cracking, the stiffness of springs subject to tension is set to be zero. The residual stresses are then redistributed in the same manner as for compression springs.

The relationship between shear stress and strain for concrete springs is assumed to be linear until the cracking of concrete. After cracking, the shear stresses drop to a level which is dependent to the friction coefficient parameter as shown in Figure 3.6. In other words, the aggregate interlock and friction at the crack surface affect the drop of the shear stresses. However, this current model cannot model the bar or anchorage slip.

To model the steel rebar, the model developed by Ristic et al. [1986] is utilized. The steel rebar model is shown in Figure 3.7. The tangent stiffness of the reinforcement spring is computed based on the strain from the reinforcement spring, loading state (loading or unloading), and the history of the reinforcement spring which controls the Bauschinger's effect. The main advantage of this model is that it can consider the effects of partial unloading and the Bauschinger's effect without any substantial complication to the analysis. After reaching $10 \%$ of strain in tension, it is assumed that the rebar fractures. The force carried by the failed rebar is then 
distributed by applying the redistributed forces to the corresponding elements in the opposite direction.

There are several assumptions in the formulation regarding the material models that can be summarized below [Tagel-Din and Meguro, 2000b]:

1. Although the post-peak process of concrete or reinforcement spring is time dependent according to the post-peak strain value, the effect is neglected. The total value of the failed spring force is redistributed during the next increment irrespective to the post-peak strain value.

2. The effects of buckling of rebars are not considered in the analysis.

3. In case of static analysis, a minimum stiffness value is assumed for concrete springs after cracking. This will result in residual tension force acting in the normal springs after cracking that will be redistributed in the next increment.

4. In case of dynamic analysis, the technique used in point 3 cannot be applied. If a minimum stiffness value is assumed at the crack location, it will affect significantly the dynamic behaviour of elements after the separation of structural members. Rigid body motion of elements also cannot be followed accurately. Therefore, spring stiffness after cracking is assumed to be zero until crack closure occurs. 
5. After reaching $1 \%$ of strain in concrete, compression springs are assumed to retain a minimum stiffness values.

\subsubsection{Failure Criteria}

In the AEM formulation of 2D element, applying Mohr-Coulomb's failure criteria calculated from normal and shear springs instead of principal stresses can lead to inaccurate simulation of fracture behaviour of the structure [Tagel-Din and Meguro, 2000b]. To resolve this problem in the AEM formulation, the occurrence of cracks is detected by using the calculated stresses around each element.

Principal stresses at each spring location are determined by the following technique as shown in Figure 3.8. The shear and normal stress components, $\tau$ and $\sigma_{1}$, at point (A) are determined from the normal and shear springs attached at the contact point location. The secondary stress, $\sigma_{2}$, at point $\mathrm{A}$ can be calculated from the normal stresses at points (B) and (C) as shown in Figure 3.8 as follows:

$$
\sigma_{2}=\frac{\mathrm{x}}{\mathrm{a}} \sigma_{\mathrm{B}}+\frac{(\mathrm{a}-\mathrm{x})}{\mathrm{a}} \sigma_{\mathrm{C}}
$$

The principal stress, in this case tensile stress, $\sigma_{\mathrm{p}}$, is then calculated as follows:

$$
\sigma_{\mathrm{p}}=\left(\frac{\sigma_{1}+\sigma_{2}}{2}\right)+\sqrt{\left(\frac{\sigma_{1}-\sigma_{2}}{2}\right)^{2}+(\tau)^{2}}
$$


If the value of the principal stress $\sigma_{\mathrm{p}}$ exceeds the critical value of tension resistance, the normal and shear spring forces are transferred to the centre of the element as a pair of force and moment. Then, these redistributed forces are applied to the structure in the next increment. The redistribution of spring forces at the crack location is very important to follow the proper crack propagation. For the normal spring, the total force is redistributed in order to have zero tension stress at the crack faces.

The shear springs at the location of tension cracking might have some resistance after cracking because of the effect of interlocking between crack faces. However, the stiffness is assumed to be zero after crack occurs. To consider the effects of friction and interlocking, a Redistributed Value (RV) as shown in Figure 3.6 is utilized. For springs subjected mainly to compressive forces, Mohr-Coulomb's failure criterion is used to determine the compression shear failure. If a spring reaches the compression shear failure, the shear force will be redistributed and shear stiffness is assumed to be zero in the following increment. It is noted that the use of RV factor is an approximation to consider the post-cracking shear behaviour [Tagel-Din and Meguro, 2000a].

Furthermore, the local crack inclination angle to the element edge direction, $\beta$, can be calculated as follows:

$$
\tan (2 \beta)=\left(\frac{2 \tau}{\sigma_{1}+\sigma_{2}}\right)
$$


Having zero shear stress means that the local crack direction coincides the element edge direction. In shear dominant zones, the stress direction is mostly affected by the shear stress value.

To represent the element behaviour after a crack occurrence, two main techniques can be used. The first technique is to break the element into two segments, as shown in Figure 3.9(a), and each of the $2 \mathrm{D}$ elements has three DOFs. In this technique, the redistribution of tensile stresses is made at the principal tensile stress plane and zero shear plane. This technique has some advantages according to TagelDin and Meguro [2000a]:

1. The redistribution of tensile stresses is accurate.

2. Crack direction inside the element can be accurately represented.

3. Crack width can be accurately calculated so shear transfer and shear softening process can be simulated as the element is separated into two at the exact crack location.

4. Compression shear type of failure can also be simulated accurately.

Despite the advantages mentioned above, this technique also has several complications [Tagel-Din and Meguro, 2000a]:

1. The number of elements will increase significantly after cracks occur, especially when the elements used are small and the number of cracks is large. 
2. Computation time will also increase after cracking. Also, the new elements will have new numbers (labels) far from the adjacent elements which will make a drastic increase in the stiffness matrix bandwidth at each crack location.

3. The spring stiffness at the cracked elements cannot be calculated as accurately as before cracking because each spring can no longer simulate a certain area like before cracking. The effect is not very big in case of monotonic loading where cracks do not close; but in case of cyclic loading where cracks can close during unloading process, this effect can lead to inaccuracy in the analysis. Having inaccurate spring stiffness can lead to inaccurate displacement and incorrect fracture behaviour.

4. Difficulties also appear in the case of cyclic loading where two main groups of cracks occur. Successive cracking of the same elements can cause a drastic decrease in the accuracy of the solution.

5. When the angle $\beta$ is small, numerical errors arise if the cracked elements have a small aspect ratio. Having small elements adjacent to large-sized elements increases the numerical errors in the analysis process. 
6. For continuation of cracks within an element, the formulation assumes newly generated cracks start from the end of previously generated cracks.

The second technique is to assume that failure inside the element is represented by failure of attached springs, as shown in Figure 3.9(b). This technique can overcome the complications of the previous technique. In this technique, if a spring fulfills the failure criterion, the following steps are taken:

1. Redistribute the spring forces.

2. Because of the force redistribution, stresses at the springs at the fracture zone surrounding the crack increase. This will make the springs near the fracture zone to fail easily.

3. Set failed springs stiffness to zero.

This technique is relatively simple and has the advantage that no special treatment is needed to represent cracking. In cases when shear stresses are not dominant, e.g., slender frames, the angle $\beta$ tends to be zero. This implies that the crack is parallel to the element edge and high accuracy can be expected. However, this technique also has a disadvantage that the crack width cannot be accurately calculated. Therefore, post-fracture behaviour parameters that depend on crack width such as shear transfer and shear softening cannot be simulated accurately. Compression shear failure also cannot be simulated accurately if the fracture plane is not parallel to the element edges. 
For 3D problems, similar approaches are used. In general, the material is assumed to crack if the major principal stress reaches the tensile strength of the material [ASI, 2006]. Figure 3.10 shows the state of stresses at each spring location and the major principal stresses in $3 \mathrm{D}$. The techniques to represent crack in the element are the same as those used in 2D problems.

\subsubsection{Element Formulation for Collision and Re-contact}

The element formulation in the AEM can consider the effects of collision and re-contact. To simplify the analysis, element shape is assumed to have circular or round shape during collision, as shown in Figure 3.11(a). This assumption is acceptable provided that the element size is relatively small. If relatively large elements were used, this assumption may still be reasonable since in the deformation range the sharp corners of the elements are broken due to the stress concentration and the edge of the elements become round [Tagel-Din and Meguro, 1999]. Based on this assumption, only the distance between the centres of the elements is measured to check the collision.

The time to check collision between elements is proportional to $(\mathrm{N} \times \mathrm{N})$, where $\mathrm{N}$ is the number of elements. The geometrical coordinate technique is used in the analysis. Every element has its space coordinates which depend on the location during analysis. These coordinates change after failure due to large geometrical changes in the structure. Using this technique, contact between elements is checked 
between the element of interest and its neighbour elements only, which will reduce the computational time.

To represent the collision, the following technique developed by Tagel-Din and Meguro [1999] is followed:

1. Solve Equation (3.18) for static loads, e.g. gravity loads, and obtain the displacements.

$$
\boldsymbol{K} \Delta \boldsymbol{U}=\Delta f_{S}
$$

where $K$ is the nonlinear stiffness matrix, $\Delta f_{s}$ is the incremental static load vector, and $\Delta U$ is the incremental displacement vector. It should also be noticed that Equation (3.18) cannot be solved if the structure is originally unstable.

2. Select a proper value of the initial time increment. Before collision occurs, longer time increment can be used.

3. Solve the equation of motion, Equation (3.8), using Newmark's Beta method to obtain the displacements.

4. Modify the structural geometry by modifying the element position and orientation according to the calculated displacement.

5. Modify the direction of the spring forces based on the new structural geometry. 
6. Calculate the element geometrical coordinates for each element.

7. Check for collision between each element and the neighbouring elements. If collision occurs, the following steps are followed:

a. The time increment is reduced to follow the material behaviour properly during collision. After separation of elements, the time increment is increased automatically to its original value.

b. Collision springs (normal and shear springs) are added between the pair of collided elements as shown in Figure 3.11(b). The normal spring direction passes through the element centroid and the shear spring direction is tangent to the assumed circle. These springs exist while the elements are in contact and will be removed after the separation of elements. When separation of elements occur, residual tension and shear forces are redistributed by applying forces in the opposite direction.

c. The global stiffness matrix is assembled in a vector form. This vector consists of the non-zero elements of half of the original global stiffness matrix. This technique will make the bandwidth associated for each DOF depends mainly on element contacts. If element contacts do not change, the 
bandwidth associated for each DOF is constant and will save the computational time because the location of each stiffness matrix element is defined just once before analysis. After collision, the sparse stiffness matrix is modified as follows:

i. The bandwidth associated with the elements in contact is determined. The bandwidth increases with the existence of new elements in contact. This increase depends mainly on the element numbering.

ii. The new position of element stiffness matrix in the global stiffness vector is recalculated because of the increase of the bandwidth. This will also increase the global stiffness vector size.

iii. The new bandwidth calculated for each DOF is utilized in the next increments until contact condition changes.

d. Collision spring stiffness is set to zero and redistribution of residual tension forces is made when separation occurs.

8. Determine the resultant spring forces, including those from collision springs, at the location of each element centroid, $F_{M}$. 
9. Determine the geometrical residuals with Equation (3.19).

$$
F_{G}=f(t)-M \Delta \ddot{U}-C \Delta \dot{U}-F_{M}
$$

10. Determine the new stiffness matrix for the new elements configuration.

11. Solve Equation (3.20) and calculate the incremental displacement, velocity, and acceleration for each element.

$$
M \Delta \ddot{U}+C \Delta \dot{U}+K \Delta U=\Delta f(t)+F_{G}
$$

In case of earthquake loading, replace the term $\Delta f(t)$ by the incremental ground acceleration matrix $-\boldsymbol{M} \Delta \ddot{\boldsymbol{U}}_{g}$.

12. Apply a new time increment and repeat steps 4 to 11 .

\subsubsection{Effects of Number of Connecting Springs between Elements}

The effect of increasing the number of connecting springs at element interface is more apparent in the nonlinear analysis where it can lead to better results in the modelling of crack propagation in the structure. In linear analysis, the effect is also observed during the calculation of the rotational stiffness in Equation (3.5), which directly uses number of springs in the formulation. Meguro and Tagel-Din [2000, 2001] have calculated the error obtained from the calculated rotational stiffness compared to those from theoretical stiffness and have shown that the error 
ranges from $0.3 \%$ for $2 \mathrm{D}$ elements with twenty springs to $25 \%$ for $2 \mathrm{D}$ elements with only two springs. Meguro and Tagel-Din $[2000,2001]$ have further explained that the effect becomes important if the element size is relatively large compared to the structure size since the relative rotation between adjacent elements is also large. However, if smaller element size is used, the error is smaller and negligible.

\subsubsection{Effects of Element Size}

To demonstrate the effect of element size, Meguro and Tagel-Din [2000, 2001] have analyzed 2D cantilever models subjected to lateral load. The results show that structures with large-size elements tend to have higher stiffness, which leads to smaller calculated displacements, and higher failure load. Using large-size elements can also affect the shear stress distribution in the structure. The shear stress distribution becomes closer to the theoretical values as the number of elements increases (or the element size is smaller) [Meguro and Tagel-Din, 2000, 2001]. Therefore, large-size elements are only good if the structure has minor shear stresses, such as slender frames.

\subsubsection{Effects of Poisson's Ratio}

In the AEM, the effect of Poisson's ratio is shown for 2D problems [Meguro and Tagel-Din, 2000]. To include the Poisson's ratio effect, there are two approaches 
that can be taken. The first approach is to add two more DOFs in each element (one is relative deformation between the vertical edges and the other is relative deformation between the horizontal edges). However, other than causing the increase of the number of DOFs, implementing this approach can cause a coupling effect among the DOFs representing the rigid deformations of elements (initial DOFs) and DOFs representing the relative deformations (newly added DOFs). The second approach, which is adopted in the AEM procedure, is by using only the initial three DOFs. This can be done since although the elements move as rigid bodies but the assembly of elements is deformable. This approach correlates the stiffness matrix of each element to those of adjacent elements. The procedure of this approach is explained further in the reference [Meguro and Tagel-Din, 2000].

Nonetheless, for 3D problems, Poisson's ratio is not taken into account into the formulations. It is argued that Poisson's ratio effect is relatively small for most problems and including this effect will cause an increase in time, especially for 3D problems, since the procedure is longer. In addition, most methods using discrete material assumption (such as the DEM) which does not include Poisson's ratio effect in the formulation. It is noted that the FEM also does not include Poisson's ratio effect after cracking occurs in the structure. 


\subsection{Application of the Applied Element Method}

Extreme Loading for Structures (ELS) is a computer software developed by the Applied Science International, LLC that is based on the AEM formulations. In this section, the basic assumptions and features of ELS are discussed.

\subsubsection{Element Generation}

In ELS, each element has 3D physical coordinates and shape. Hence, the elements are a group of 3D elements which can be separated and/or collided together. The DOFs of each element are calculated at the centre of gravity of the element. For 3D elements, as shown in Figure 3.12, one normal and two shear springs are located on the element surfaces. Each set of springs completely represents stresses and deformations of a volume.

While the FEM elements are connected at nodes, the AEM elements are connected using the element's entire surface (element's faces) through a series of connection springs. This connection allows AEM elements to have partial connectivity during analysis. In partial connectivity case, some springs fail while the others are still active and effective. This means that elements sharing the same surface will have connectivity springs even if the shared surfaces are only a portion of it. This is useful especially to model progressive collapse of structures where failure occurs first as a local failure. Moreover, the separation of springs does not cause singularity problem so the analysis can still continue after separation. In the FEM, 
partial connectivity can be modelled by more nodes at the location of contacts which will result in more DOFs and more complication in the analysis. The comparison of partial connectivity in the FEM and the AEM is shown in Figure 3.13.

It is usually needed that some parts of the analyzed structure have smaller mesh size because of stress concentration and/or smaller crack spacing. In the FEM, the transition between elements with different sizes should be done through special meshing techniques to assure the connectivity. This can cause an increase in the number of elements and complication in meshing process. Since the AEM elements are not connected at nodes, no transition elements are needed to connect large-sized elements with smaller-sized elements. This problem is illustrated in Figure 3.14.

Each 3D element has six DOFs, three translations and three rotations, and the deformations are also related to those six DOFs as shown in Figure 3.15. The element self weight is assumed to always apply in the z-direction. In ELS, the user can select the active DOFs before the analysis. The selection of the active DOFs depends on the problem, e.g., a 2D problem in X-Z plane will have Ux, Uz, and Ry as the active DOFs.

\subsubsection{Connectivity Springs}

The generation of connectivity springs can be automatically done in ELS. The springs represent the continuity between elements. The states of stresses, strains, and failure criteria are calculated at the springs. In ELS, connectivity springs are 
divided into two groups: matrix springs and reinforcement springs. Matrix springs connect adjacent elements and represent the main structural material, i.e., concrete in reinforced concrete structure. Reinforcement springs represent the steel rebar in reinforced concrete structure.

Matrix springs can represent all material type and properties defined by the user. When the average strain between two adjacent faces reaches a specified limit, the springs between these two faces are removed and it is assumed that the elements behave as rigid body afterwards, as shown in Figure 3.16. If these elements meet again, it is considered as a contact between rigid bodies. At every calculation point, three springs (one normal and two shear springs, as shown in Figure 3.16) are assigned to represent the material behaviour as matrix springs as discussed in the previous section. The average normal strain is calculated by taking the average of the absolute normal strains on the element faces. It is noted that to generate springs between element faces, the faces should be in the same plane.

The reinforcement springs have the same material properties, exact location, and dimension of the represented rebar. Similar to matrix springs, three springs are set at the intersection of the rebar and the element boundary, as shown in Figure 3.17. The normal spring direction is taken as the direction of the rebar irrespective to the element face direction. The reinforcement springs are considered to rupture under the following conditions: 
1. The rebar stresses reach the failure criterion, when the normal stress is equal or greater than the ultimate stress. The rebar rupture only applies to bars in tension. No cut is permitted for bars in compression.

2. The matrix springs reach the separation strain limit. In this case both matrix and reinforcement springs, either in compression or tension, are removed.

\subsubsection{Material Properties}

Material properties are defined for reinforced concrete material as follows in ELS:

1. Young's Modulus.

2. Shear Modulus.

3. Tensile Strength.

4. Compressive Strength.

5. Ultimate Strength to Yield Stress Ratio.

6. Separation Strain.

7. Friction Coefficient. 
8. Specific Mass.

9. Damping Factor.

10. Post-Yield Stiffness Ratio.

Young's modulus, $E$, is defined as the ratio between longitudinal stress and strain in the elastic range for 1D frame elements, as shown in Figure 3.18.

Shear modulus, $G$, is the ratio between shear stress and strain in the elastic range for $2 \mathrm{D}$ elements, as shown in Figure 3.19. The value of shear modulus is defined as follows:

$$
G=\frac{E}{2(1+v)}
$$

where $E$ is the Young's modulus and $v$ is the Poisson's ratio.

Tensile strength is the stress value at which the element starts to rupture (tensile failure). For steel, tensile strength is known as tensile yield stress.

Compressive strength, similar to tensile strength, is the stress value at which the element starts to crush (compressive failure). For steel, compressive strength is known as compressive yield stress.

Ultimate strength to yield stress ratio is only used for steel, as shown in Figure 3.20. 
Separation strain is defined as the strain at which adjacent elements are totally separated at the connecting face, as shown in Figure 3.21. In case of elements re-contact after separation, the elements will behave as rigid bodies and contact springs will then be activated. The illustration of element separation and re-contact is presented in Figure 3.22. For steel, this value has no effect since steel rebars are cut when the stress reaches the ultimate stress or the concrete reaches the separation strain. It is suggested to use a value between 0.1 and 0.2 for separation strain [ASI, 2006].

Friction coefficient value is only used after elements have separated and recontact occurs, as shown in Figure 3.23. This is the value of the roughness of the material.

Specific mass, is defined as the mass per unit volume. If specific mass is set to zero, gravity load due to weight is neglected. In ELS, the self weight of the rebar is neglected.

Damping factor, $r$, represents the energy dissipation. To calculate the damping factor corresponding to a certain damping ratio, $\xi$, the steps below should be followed:

1. Estimate a reasonable natural period, $T$.

2. Calculate the natural frequency, $\omega$, as:

$$
\omega=\frac{2 \pi}{T}
$$


3. Estimate the damping factor, $r$, as:

$$
r=2 \xi \omega
$$

Damping factor in ELS has the initial value set to be zero. For nonlinear analysis, the assumption of zero damping value can still give good accuracy since the effect of internal damping as explained in Section 3.1.3 is taken into account in the formulation. It is also emphasized that external damping causes contributes as deceleration force for falling objects.

Post-yield stiffness ratio is defined as the ratio between post-yield and preyield tangent moduli of steel, as shown in Figure 3.24. This value is only utilized for steel material such as steel rebar. The default value for post-yield stiffness ratio in ELS is set to be 0.01 .

\subsubsection{Contact Parameters}

After contact occurs between elements, contact springs are generated at contact points. These contact springs are linear springs which transfer energy between elements. Contact spring stiffness should be assigned with a reasonable value. Very high contact stiffness value causes large force to transfer between elements. On the other hand, low contact stiffness value causes underestimation of transmitted force when elements collide. To define the contact spring stiffness, there are three parameters to be defined: 
1. Normal Contact Stiffness Factor.

For elements in contact, Equation (3.1.a) can be rewritten as:

$$
K_{n}^{*}=\frac{E A}{d}
$$

where $K_{n}^{*}$ is the contact spring normal stiffness, $E$ is the minimum value of the Young's modulus of the two elements in contact, $A$ is the average contact area between elements, and $d$ is the centre-tocentre distance between the two elements in contact which is assumed to be a constant value calculated from the average element size.

Assuming that $d$ is the ratio of the average contact area, one will have:

$$
A=F_{N} d^{2}
$$

where $F_{N}$ is the normal contact stiffness factor.

Finally, by substituting Equation (3.26) to Equation (3.25) one will obtain:

$$
K_{n}^{*}=E F_{N} d
$$

The value of $F_{N}$ can be inputted by user and the default value in ELS is 0.01 . 


\section{Shear Contact Stiffness Factor.}

Shear contact stiffness factor has similar concept with normal contact stiffness factor. However, the value is less than of that of normal contact stiffness factor since the transmitted normal force is higher than the transmitted shear force.

Following the same logic as used in normal contact stiffness factor, one will obtain contact spring shear stiffness, $K_{s}^{*}$, as follows:

$$
K_{s}^{*}=G F_{S} d
$$

where $F_{S}$ is the shear contact stiffness factor, $G$ is the minimum value of the shear modulus of the two elements in contact, $d$ is the centre-to-centre distance between the two elements in contact which is assumed to be a constant value calculated from the average element size, and $F_{S}$ is the shear stiffness factor. The value of $F_{S}$ can be inputted by user and the default value in ELS is 0.001 .

\section{Contact Spring Unloading Stiffness Factor.}

During contact, some energy is dissipated. The ratio between loading stiffness and unloading stiffness is called the contact spring unloading stiffness factor, $n$, as shown in Figure 3.25. This factor controls how much energy is dissipated during contact. 
The value of $n=1$ means that no energy is dissipated during contact and element will rebound by the same speed as before colliding. The value of $n>1$ indicates that energy dissipation exists during contact. In ELS, the default value is $n=10$ which means that the energy dissipation is $90 \%$ of the total energy and only $10 \%$ of the total energy is recovered. This value is reasonable if the user does not have a more accurate value [ASI, 2006].

\subsubsection{Restraints}

Structures are restrained against moment at support locations. The support can be rigid and/or elastic. Having rigid supports implies that the displacement should be zero at the support. Moreover, restraint at a specific joint leads to a reaction force or moment corresponding to the restrained DOF. Some common restraints that can be modelled in ELS are:

1. Fixed supports.

Fixed supports restraint all displacement and rotational components of an element. There are two ways to model fixed supports in ELS, as an example for a column: 
a. Select all elements from the column base which contact the ground and fix the displacement on $\mathrm{x}$-, $\mathrm{y}-$, and z-axes.

b. Select the centre element from the column base which contact the ground and fix the displacement on $\mathrm{x}^{-}, \mathrm{y}-$, and $\mathrm{z}-\mathrm{axes}$ and rotation about $\mathrm{x}-, \mathrm{y}-$, and z-axes.

2. Hinged supports.

Hinged supports restraint all displacement components but all rotational components are free. To model hinged supports in ELS, the user needs to fix all displacement on $x^{-}, y^{-}$, and $z$-axes. For stability, it is also suggested to fix the rotation about z-axis.

3. Roller supports.

Roller supports restraint one or two displacement components, in other words the roller is allowed to move in one or two directions only. To model roller supports in ELS, the user needs to fix the desired displacement(s). For stability, it is also suggested to fix the rotation about z-axis. 


\subsubsection{Loadings}

ELS can perform static and dynamic analyses with load or displacement control. Types of loadings that can be inputted to ELS are as follows:

1. Static Loadings
a. Self Weight
b. Lumped Weight (or Mass)

2. Dynamic Loadings
a. Earthquake Loads
b. Inertia Loads from Lumped Mass
c. Blast Loads
d. Element Removal
e. Pressure Loads

The earthquake loads can be inputted as acceleration time history or displacement time history. 


\subsubsection{Analysis Solver}

Generally, analysis is done to solve the equation of motion as mentioned in Equation (3.3), Equation (3.4), Equation (3.6), Equation (3.9), or any variation of those equations. Furthermore, analysis is also done to solve the eigenvalue problem as stated in Equation (3.11). The solution for static problems is more straightforward. The solution for dynamic problems needs numerical iteration procedure. In ELS, the Newmark's Beta method is adopted to solve the equation of motion in dynamic problems.

The equation of motion is a linear system of equations for each step. The solution of this linear system can be done with several algorithms. In ELS, the equations are solved using either the direct solver or the iterative solver. The direct solver uses the Choleski factorization with upper-lower decomposition with modification using CXML algorithm [ASI, 2006]. The iterative solver is by and large faster than the direct solver especially for a large number of DOFs. For more detail on the algorithms of the analysis solvers, one can refer to the ELS Technical Manual [ASI, 2006].

The direct solver is ideal for static or dynamic analysis with relatively large time increment, while the iterative solver is ideal for dynamic analysis with very small time increment. ELS can test both solver and switch between both solvers automatically to find the fastest solution. When the analysis switches between static and dynamic stages, the most optimum solver is tested and utilized. 


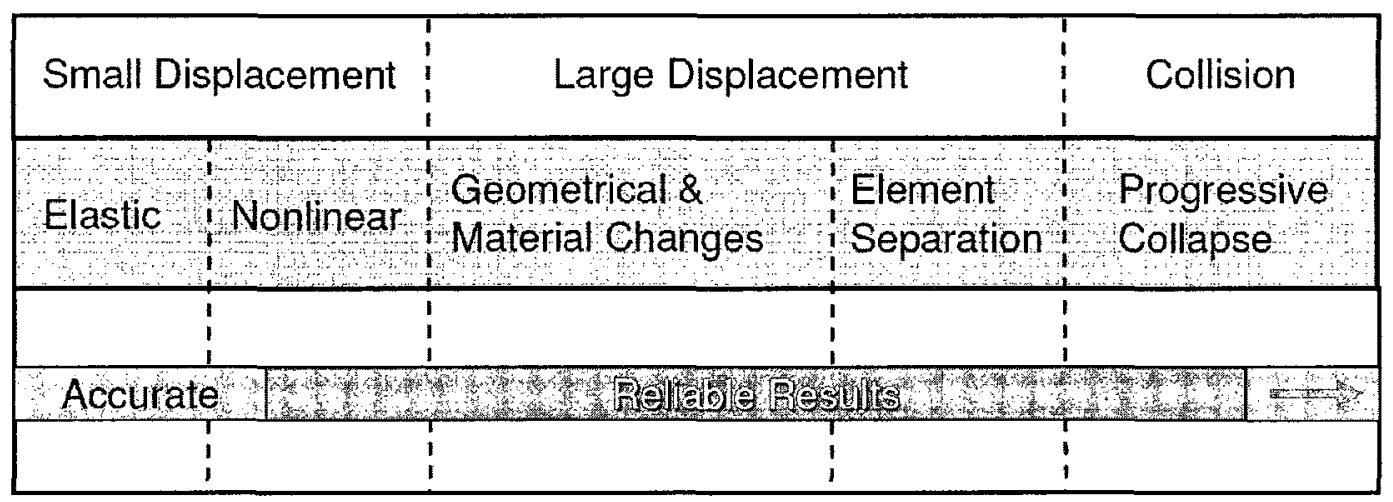

Figure 3.1. Analysis Domain of the Applied Element Method [ASI, 2006] 


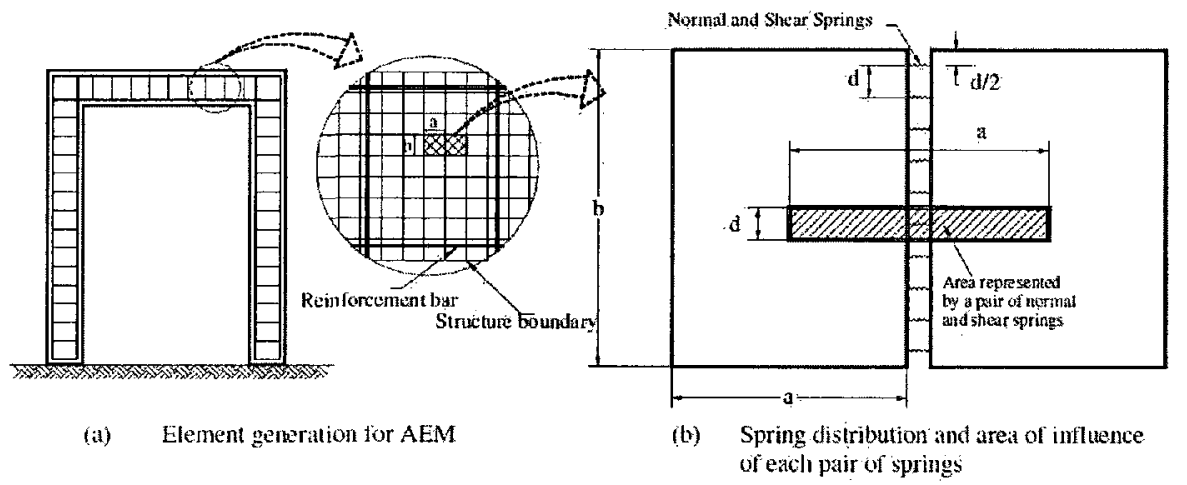

Figure 3.2. Elements on the Applied Element Method [Meguro and Tagel-Din, 2000] 


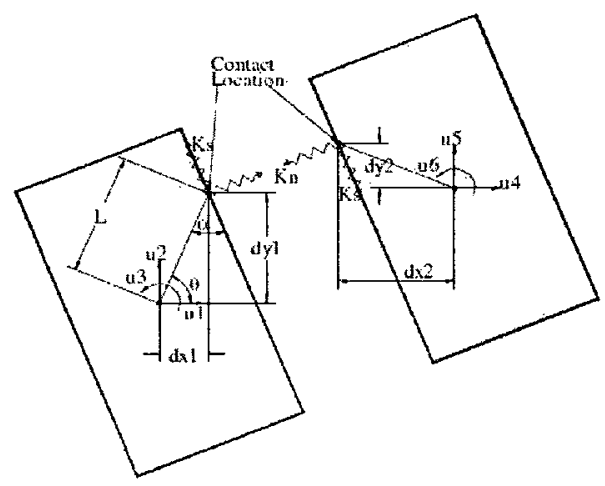

Figure 3.3. Element Contact Point and Degrees of Freedom [Meguro and Tagel-Din, 2000] 


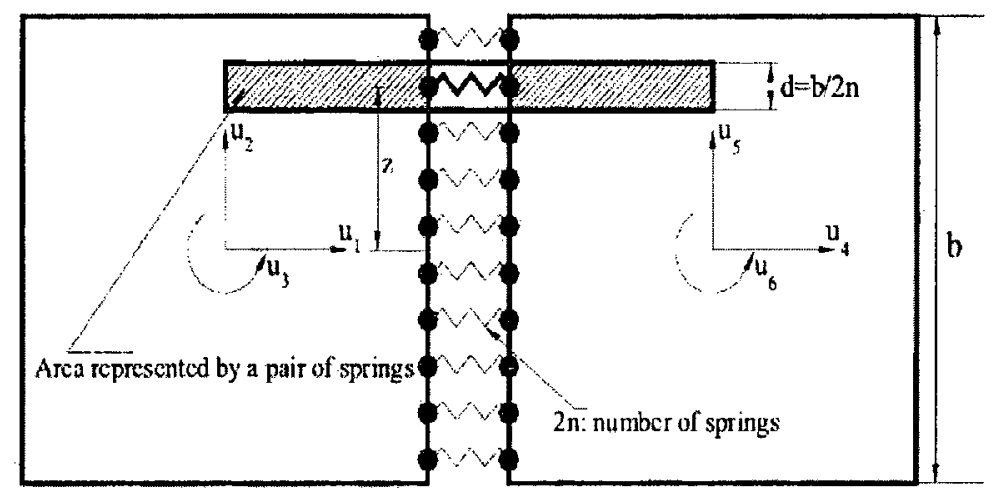

Figure 3.4. Normal Springs for Rotational Stiffness Calculation [Meguro and Tagel-

Din, 2001] 


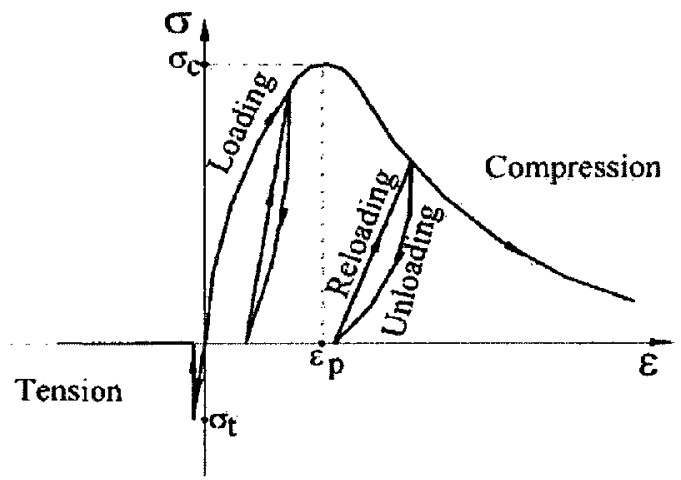

Figure 3.5. Maekawa Concrete Model [Modified by Meguro and Tagel-Din, 2001] 


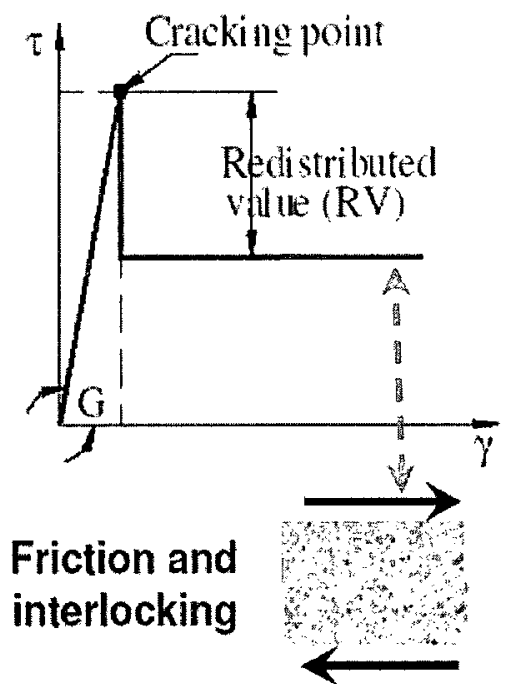

Figure 3.6. Model for Concrete under Shear Stress [ASI, 2006] 


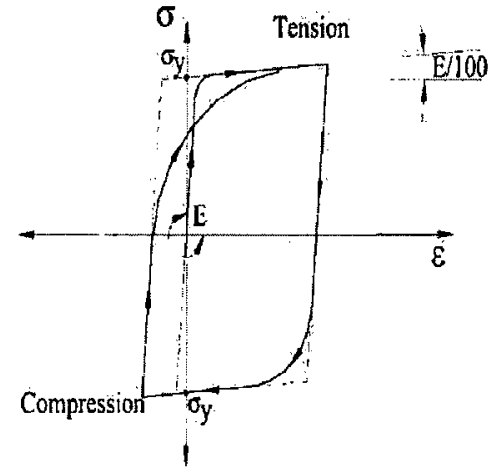

Figure 3.7. Ristic Steel Model 


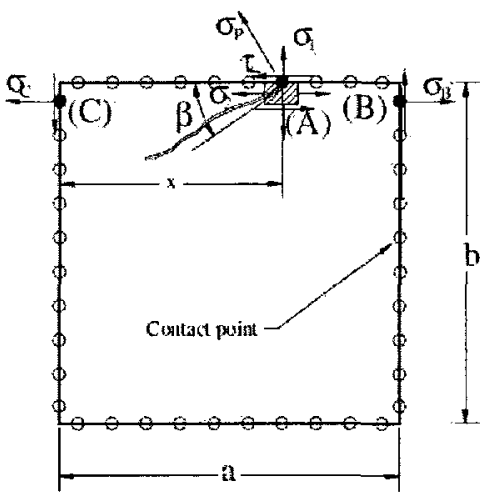

Figure 3.8. Principal Stresses for 2D Problems [Tagel-Din and Meguro, 2000a] 

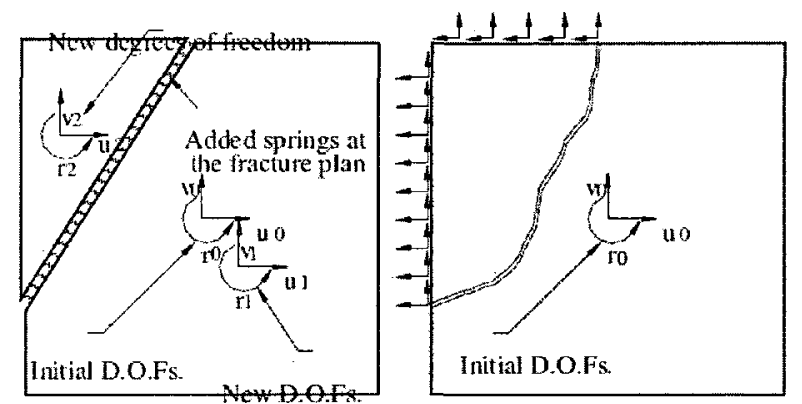

a) Redistribution of springs

b) Redistribution of springs forces at the crack faces forces at the element edge

Figure 3.9. Strategies for Element Cracking [Tagel-Din and Meguro, 2000a] 


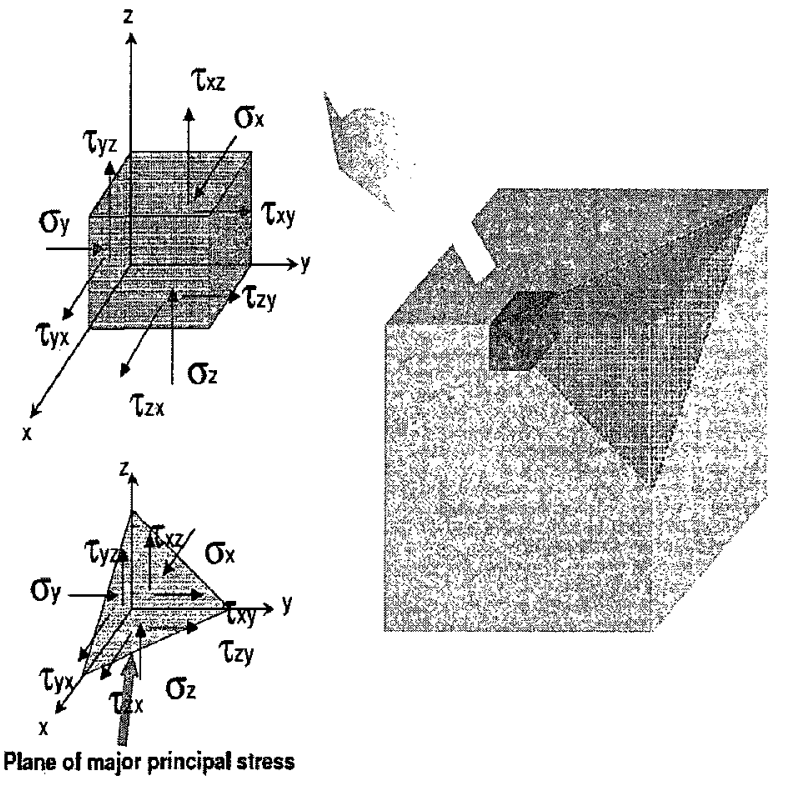

Figure 3.10. Principal Stresses for 3D Problems [ASI, 2006] 


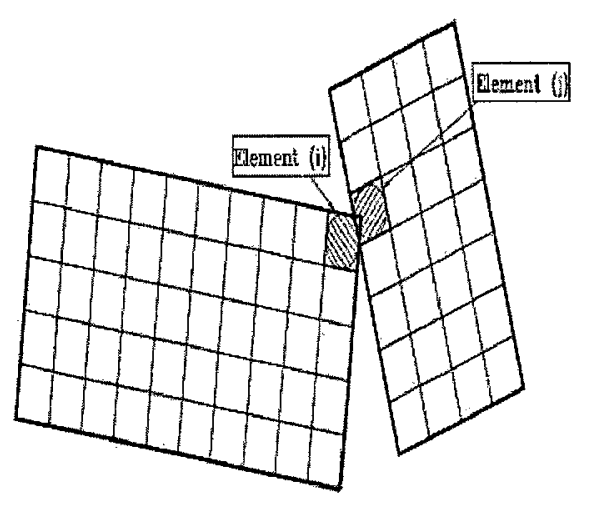

(a)

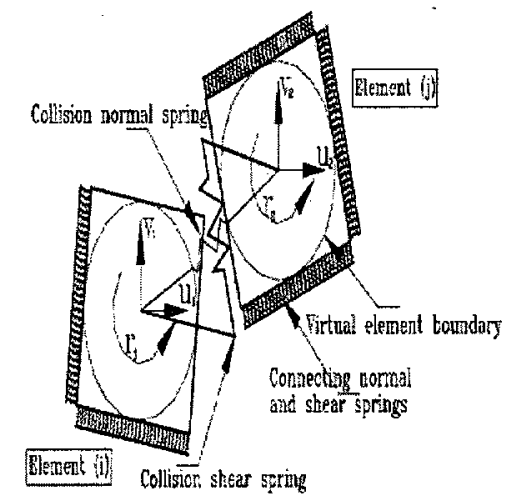

(b)

Figure 3.11. (a) Collision of Elements and (b) Springs Generated during Collision

[Tagel-Din and Meguro, 1999] 


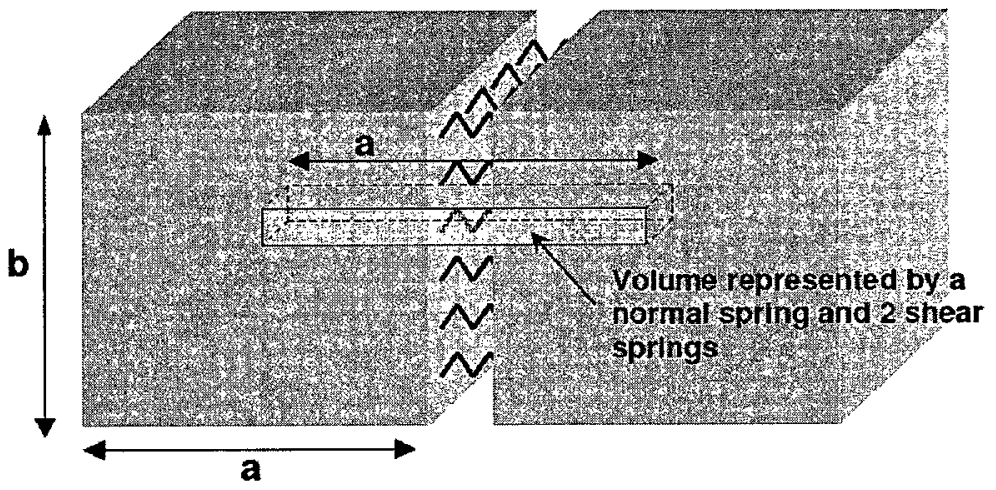

Figure 3.12. Elements in ELS [ASI, 2006] 

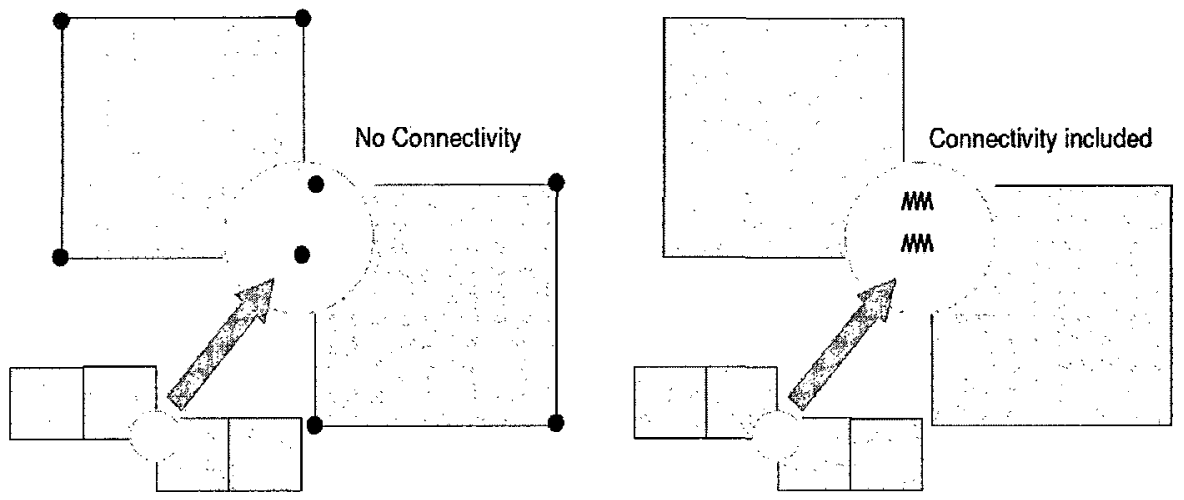

Figure 3.13. Partial Connectivity in (a) FEM and (b) AEM [ASI, 2006] 

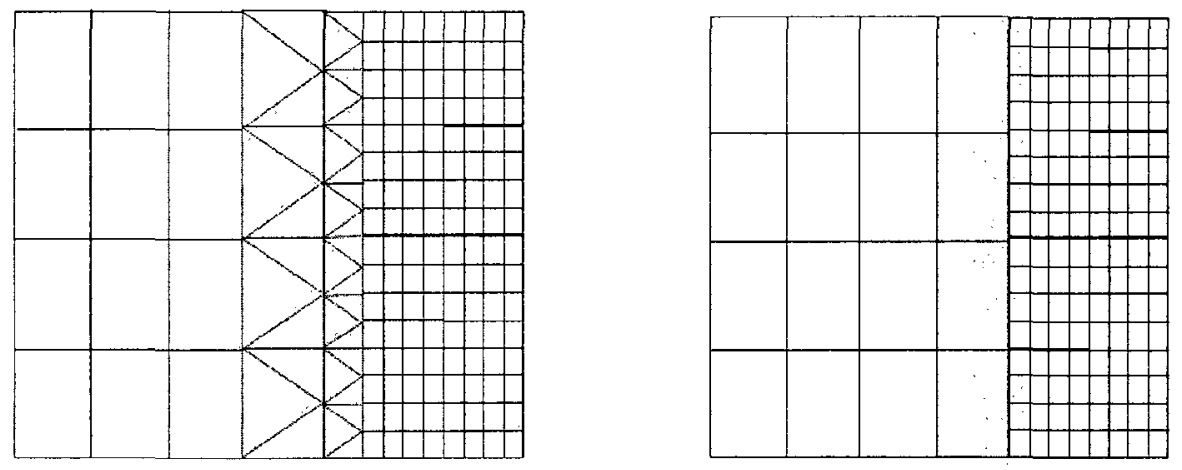

Figure 3.14. Comparison of Mesh in (a) FEM and (b) AEM [ASI, 2006] 


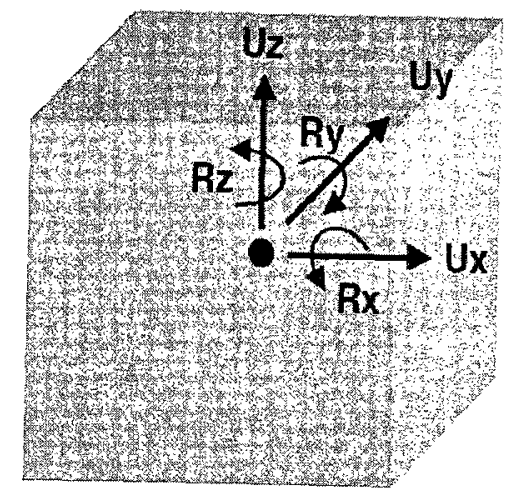

(a)
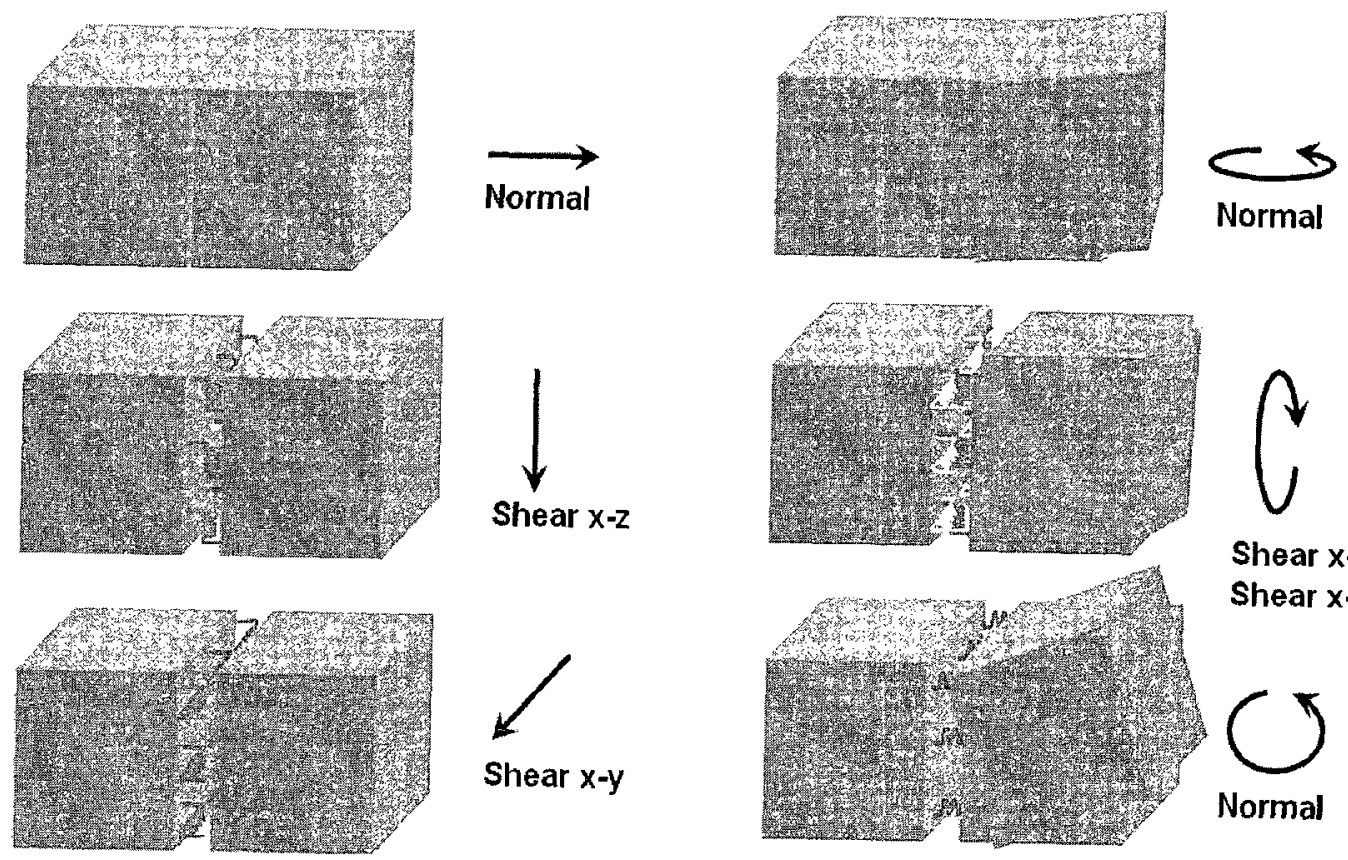

Translation

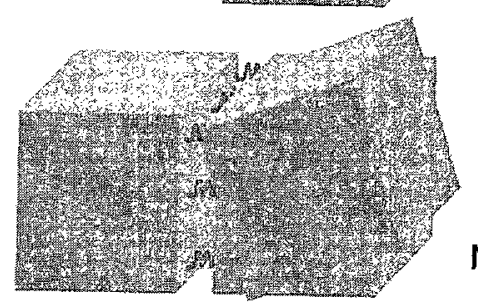

Shear $x-z$ Shear $x-y$

Rotation

(b)

Figure 3.15. (a) 3D Element's Degrees of Freedom and (b) Deformations Related to the Degrees of Freedom [ASI, 2006] 


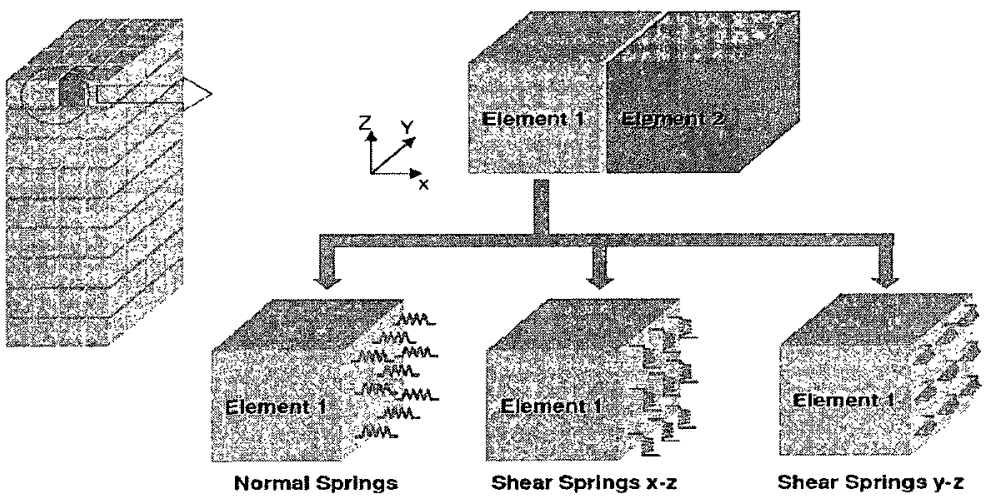

Figure 3.16. Matrix Springs between Elements [ASI, 2006] 


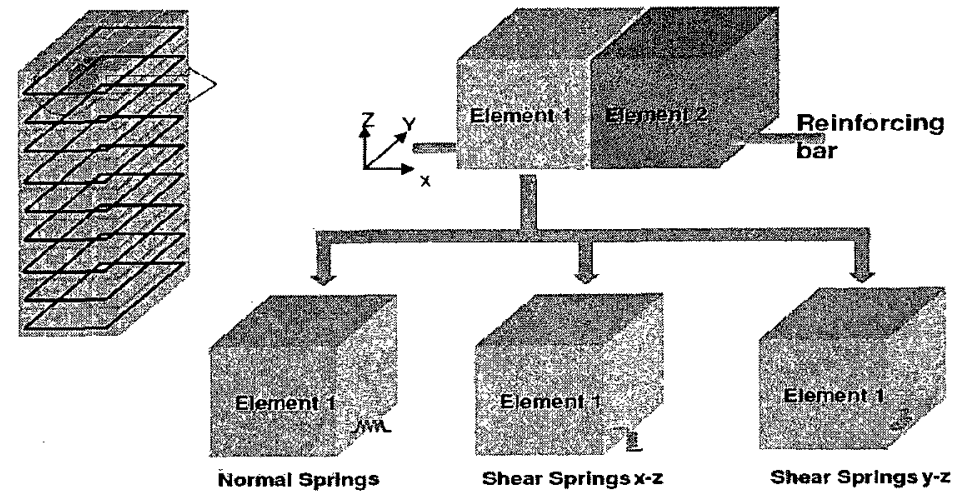

Figure 3.17. Rebar Springs between Elements [ASI, 2006] 


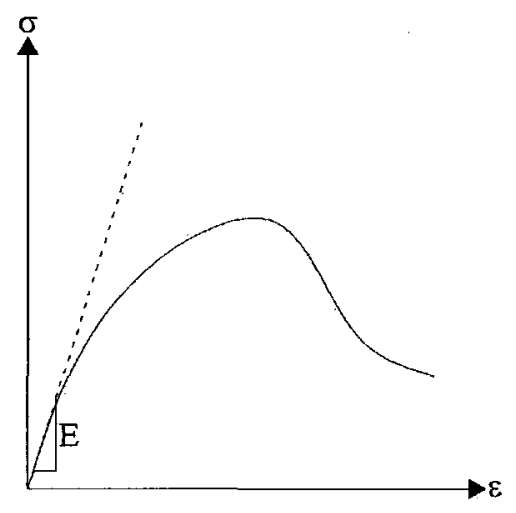

(a)

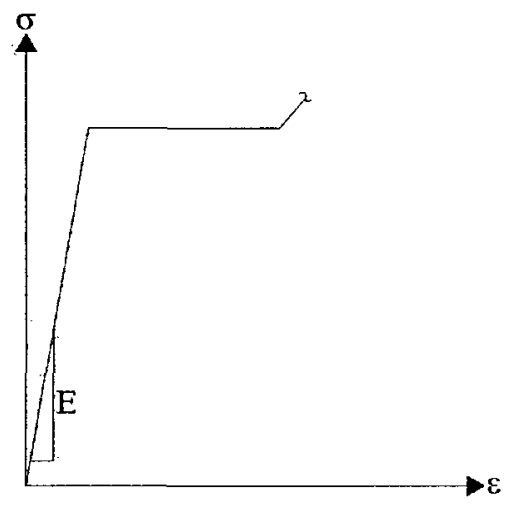

(b)

Figure 3.18. Illustration of Young's Modulus of (a) Concrete and (b) Steel 


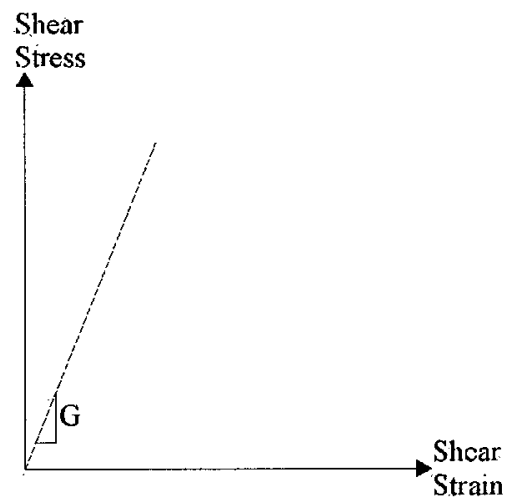

Figure 3.19. Illustration of Shear Modulus 


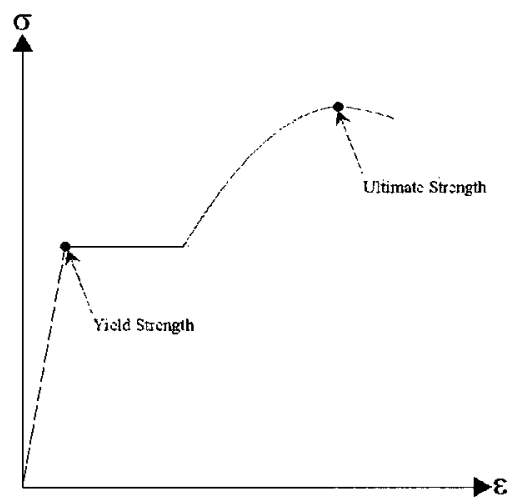

Figure 3.20. Illustration of Yield and Ultimate Stresses of Rebar 

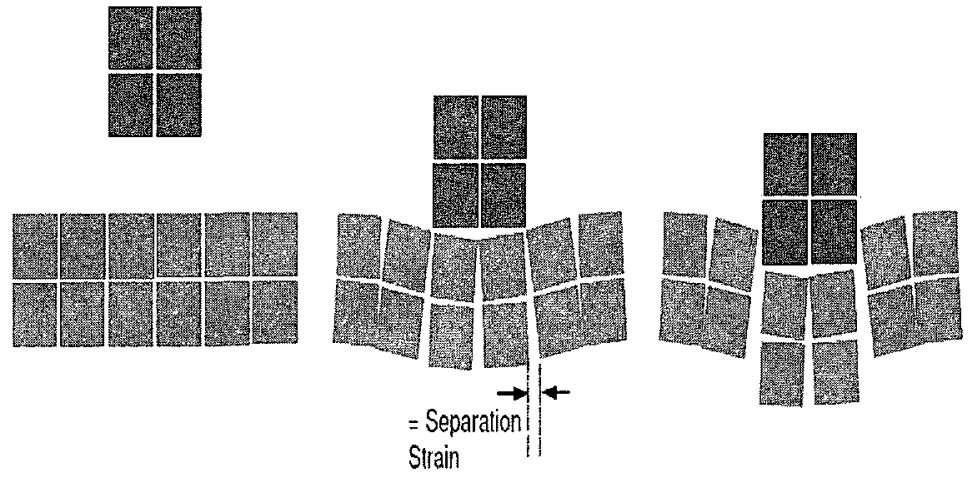

Figure 3.21. Illustration of Separation Strain [ASI, 2006] 


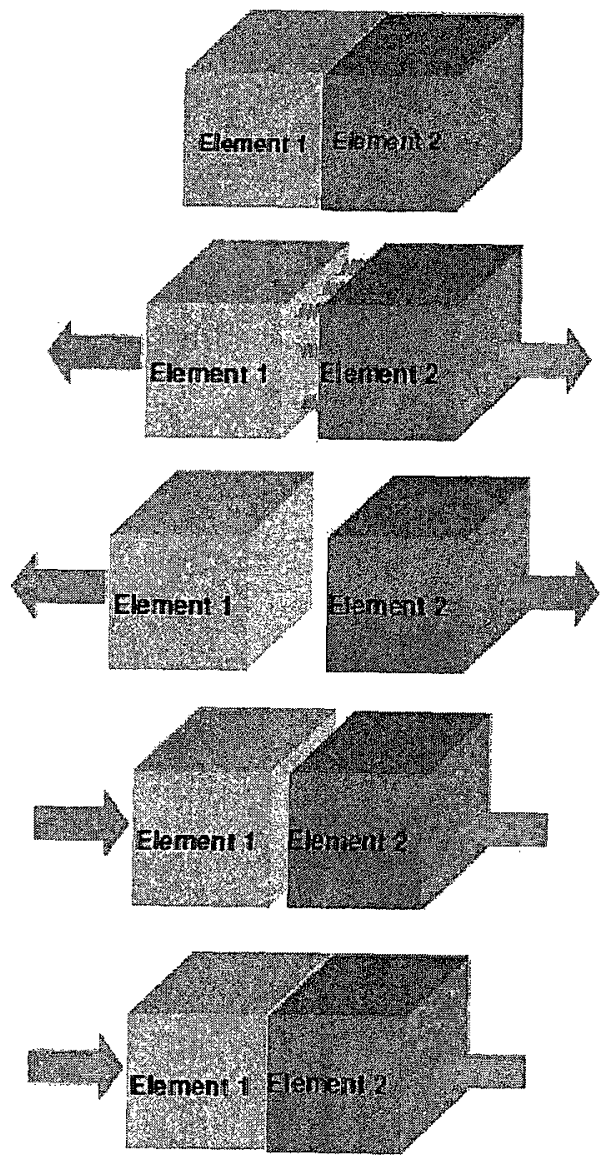

Initial position

Matrix springs under tension

Separation strain reached

Elements forced towards each other (according to loading condition)

Contact springs activated

Figure 3.22. Illustration of Element Separation and Re-contact [ASI, 2006] 

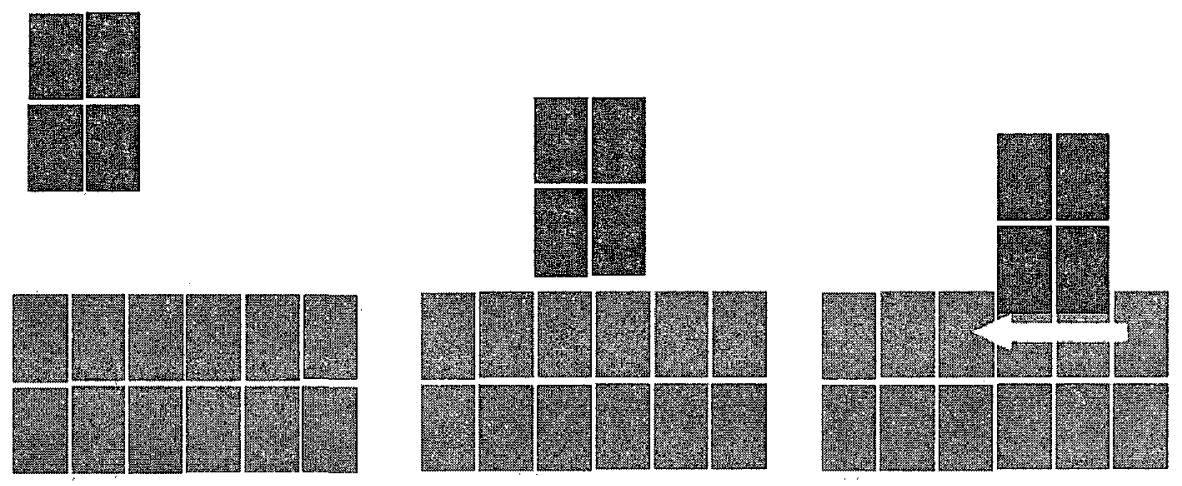

Figure 3.23. Illustration of Element Friction [ASI, 2006] 


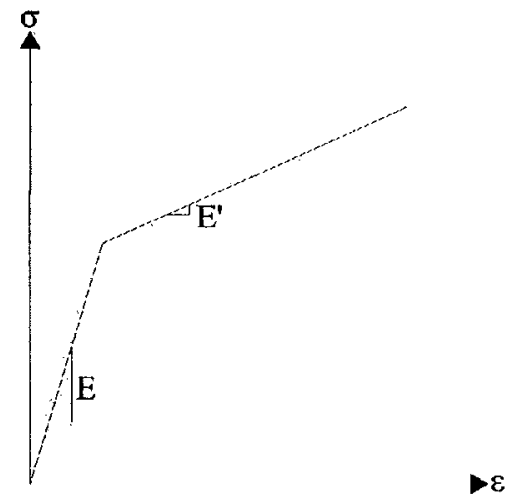

Figure 3.24. Illustration of Post-yield Stiffness Ratio 


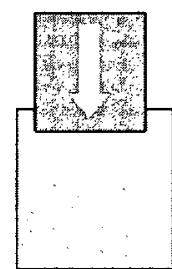

a

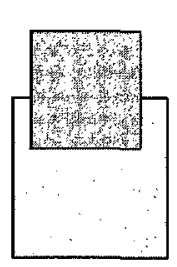

b
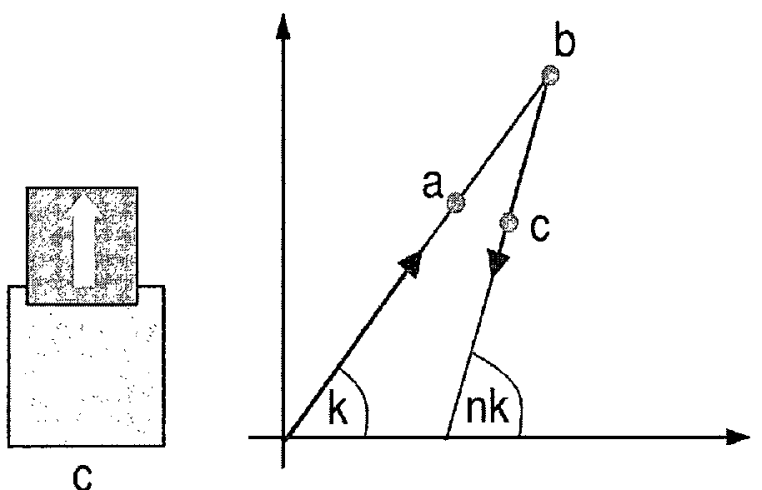

Figure 3.25. Illustration of Contact Spring Unloading Stiffness Factor [ASI, 2006] 
CHAPTER 4

\section{Bridge Models and Ground Accelerations}

This chapter presents the bridge models analyzed for the progressive collapse behaviour in this research. The bridge models used are examples of shortand medium-span bridges. This chapter also presents the earthquake records used for the time history analysis.

\subsection{Bridge Models}

The bridge models selected in this research have been adapted from previously investigated bridge models by Casarotti et al. [2005] and Casarotti and Pinho [2006, 2007]. In the literature, the longitudinal reinforcements of the bridge piers are given. The details of the transverse reinforcements and the reinforcement details of the box girder are not known as equivalent beam model is presented in the references [Casarotti and Pinho, 2006, 2007]. In ELS, it is assumed that the reinforcement details in the box girder and the transverse reinforcement details in the piers are obtained from specifications for reinforcement form the Canadian Highway Bridge Design Code (CAN/CSA-S6-06) [CSA, 2006]. 


\subsubsection{Overview of the Short-Span Bridge Model}

The bridge has four $20 \mathrm{~m}$ bays with a total length of $80 \mathrm{~m}$. The bridge superstructure dimensions and pier heights of $5.6 \mathrm{~m}, 2.8 \mathrm{~m}$, and $8.4 \mathrm{~m}$ respectively, are shown in Figure 4.1. The pier base is modelled as fixed at the pier bases (Piers 2 to 4) and hinged at locations 1 and 5. The longitudinal reinforcement details of the bridge are taken from the bridge model by Casarotti and Pinho [2006]. However, the transverse reinforcement details of the piers are determined for the study here based on the design requirements of CAN/CSA-S6-06 [CSA, 2006]. The bridge superstructure is a box girder $5.6 \mathrm{~m}$ wide and in this study, the girder is considered as reinforced concrete girder. The bridge box girder has minimum transverse reinforcements. The bridge substructures are rectangular hollow-core reinforced concrete piers. The bridge's piers properties are summarized in Table 4.1. In the applied element model, the bridge box girder is modelled to remain elastic during earthquake response in the comparison study with previous analytical and experimental test results where elastic bridge superstructure is assumed [Casarotti and Pinho, 2006]. The concrete and steel properties are tabulated in Table 4.2. The bridge model is shown in Figures 4.1 and 4.2. The damping ratio of the bridge in the analysis is taken as $5 \%$.

The longitudinal reinforcement details of the bridge box girder are shown in Figure 4.3. The box girder is designed such that it matches the moment of inertia specified in the literature [Casarotti and Pinho, 2006]. The piers' longitudinal reinforcement details are divided into two types. The $2.8 \mathrm{~m}$ high pier follows the 
reinforcement detail type 1 , whereas the $5.6 \mathrm{~m}$ high and $8.4 \mathrm{~m}$ high piers follow the reinforcement detail type 2 . The longitudinal reinforcement details of the piers are shown in Figure 4.4.

In the applied element model of the bridge, each side of the pier crosssection is divided into a mesh of $5 \times 5$ equal spaced elements and in the longitudinal direction into 5 equal spaced elements per $2.8 \mathrm{~m}$ length. Each surface area of the box girder section (i.e., the deck, webs, and soffit) is divided into $10 \times 1$ elements with 50 elements per $20 \mathrm{~m}$ length in the longitudinal direction of the box girder. This mesh size has been found to give accurate results. Analysis using a finer mesh has been carried out without any noticeable difference in the numerical results.

The connection between the pier and the box girder is assumed as pin, which does not transfer any moment from the box girder to the pier. This pin connection in the finite element model by Casarotti and Pinho [2006] is idealized by releasing the moment at the particular point. In the applied element model, the detailed structural configuration is modelled since it cannot model a release. A simple model is introduced as shown in Figure 4.5 to model a pin connection. A base plate, divided into $11 \times 3 \times 1$ elements, with a thickness of $5 \mathrm{~cm}$ is used to model a cap on top of the pier and a small element with size of $5 \times 5 \times 5 \mathrm{~cm}$ is modelled to accommodate the element rotation about the longitudinal axis of the pier. The longitudinal reinforcement from the pier is also assumed to be terminated at the soffit face. This model is then verified to have no moment transferred from the box girder to the pier for the case of gravity loads. 


\subsubsection{Overview of the Medium-Span Bridge Model}

The bridge has eight $20 \mathrm{~m}$ bays with a total length of $160 \mathrm{~m}$. The bridge superstructure dimensions and pier heights of $5.6 \mathrm{~m}, 8.4 \mathrm{~m}, 8.4 \mathrm{~m}, 2.8 \mathrm{~m}, 8.4 \mathrm{~m}, 2.8$ $\mathrm{m}$, and $5.6 \mathrm{~m}$, respectively are shown in Figure 4.6. The pier base is modelled as fixed at the pier bases (Piers B to $\mathrm{H}$ ) and the abutment supports are hinged at locations A and I. The longitudinal reinforcement details of the bridge are taken from the bridge model by Casarotti and Pinho [2006]. However, the transverse reinforcement details of the piers considered here are based on the design requirements of CAN/CSA-S6-06 [CSA, 2006]. The bridge superstructure is a box girder $5.6 \mathrm{~m}$ wide and in this study, the girder is considered as reinforced concrete girder. The bridge box girder has minimum transverse reinforcements. The bridge substructures are rectangular hollow-core reinforced concrete piers. The bridge properties are summarized in Table 4.3. The bridge model is shown in Figures 4.6 and 4.7. The damping ratio of the bridge in the analysis is taken as $5 \%$.

The longitudinal reinforcement details of the bridge box girder are the same as those shown in Figure 4.3, with exception that the top flange reinforcement is reduced to half. The piers' longitudinal reinforcement details are divided into two types. The $2.8 \mathrm{~m}$ high pier follows the reinforcement detail type 1 , whereas the $5.6 \mathrm{~m}$ high and $8.4 \mathrm{~m}$ high piers follow the reinforcement detail type 2 . The longitudinal reinforcement details of the piers are shown in Figure 4.4. In the applied element 
model of the bridge, the mesh size and arrangements follow the same mesh details in the case of the short-span bridge model discussed earlier.

\subsubsection{Transverse Reinforcements}

The transverse reinforcement in the column is designed in accordance to the specifications in the Canadian Highway Bridge Design Code (CAN/CSA-S6-06) [CSA, 2006]. There are two clauses in CAN/CSA-S6-06 [CSA, 2006] related to transverse reinforcement in bridge column. Clause 4.7.4.2.6 states that:

"Transverse reinforcement for confinement shall be provided in the plastic hinge regions specified in Clause 4.7.4.2.4 and shall extend into the top and bottom connections in accordance with Clause 4.7.4.4. The centre-to-centre spacing shall not exceed the smallest of 0.25 times the minimum component dimension, six times the diameter of the longitudinal reinforcement, or 150 $\mathrm{mm}$. The centre-to-centre spacing of interlocking spirals or hoop cages in oblong columns shall not be greater than 0.75 times the diameter of the spiral or hoop cage. A minimum of four vertical bars shall be located within each overlapping region of the spirals or hoops."

Clause 4.7.4.2.4(b) specifies the plastic hinge regions as follows:

"The plastic hinge region shall be assumed to extend from the soffit of girders or cap beams at the top of columns to the top of foundations at the bottom of columns. This distance shall be taken as the greatest of 
(i) the maximum cross-sectional dimension of the column;

(ii) one-sixth of the clear height of the column; or

(iii) $450 \mathrm{~mm}$ "

The two clauses mentioned above are applied to determine the plastic hinge region and the transversal reinforcement of the pier of the bridge models.

The plastic hinge region of the pier is taken as the greatest of: (i) $1600 \mathrm{~mm}$, (ii) $1400 \mathrm{~mm}$ if the tallest pier is used, and (iii) $450 \mathrm{~m}$. Therefore, the plastic regions on the pier are located at $1.6 \mathrm{~m}$ from the top and $1.6 \mathrm{~m}$ from the bottom of the pier. In the plastic hinge regions, the transverse reinforcement spacing is the smallest of: (i) $200 \mathrm{~mm}$, (ii) $84 \mathrm{~mm}$, and (iii) $150 \mathrm{~mm}$. In this case, the transverse reinforcement spacing in the plastic hinge region is chosen to be $80 \mathrm{~mm}$ for the sake of simplicity. Outside the plastic hinge regions the spacing is increased to be doubled of the spacing in the plastic hinge regions so it becomes $160 \mathrm{~mm}$. It is also noted that in the shortest pier, plastic regions spacing is used throughout the pier's height. The diameter of the transverse reinforcement is chosen to be $8 \mathrm{~mm}$.

\subsection{Ground Accelerations}

There are five ground accelerations used in this study. The first two ground motions are used in the verification examples and the other three are used in the progressive collapse analysis of the bridge models. The Northridge, Kobe, and ChiChi earthquake ground motions are selected because there were some bridge collapses during those earthquakes [Mitchell et al., 1995; Anderson et al., 1996; 
Wallace et al., 2001; Hsu and Fu, 2004]. The latter three ground motions are obtained from the Pacific Earthquake Engineering Research (PEER) Strong Motion Database. A summary of the earthquake ground motions used in this research is presented in Table 4.4.

The frequency content of the earthquake ground motion is examined by its response spectrum. The response spectrum is useful to obtain insights on the peak response of the structure at a particular modal period. However, this method is based on the peak response of a set of Single Degree of Freedom (SDOF) structures. Hence, although the peak response at a particular modal period is known, the total response of the structure still needs to be investigated by the nonlinear time history analysis.

\subsubsection{Artificial Earthquake Ground Acceleration from Literature}

The earthquake record was used in the analyses by Casarotti et al. [2005] and Casarotti and Pinho [2006]. This earthquake ground acceleration was taken from the thesis by Guedes [1997]. The peak ground acceleration of this earthquake record is $1.05 \mathrm{~g}$, where $\mathrm{g}$ is the gravitational acceleration. This earthquake is selected on the basis of the estimated capacity of the bridge and therefore it is increased from the design earthquake by a factor of 1.2 [Casarotti and Pinho, 2006]. The artificial earthquake record is shown in Figure 4.8. This earthquake is applied in the transversal direction of the bridge model. 


\subsubsection{Modified Kobe Earthquake Ground Acceleration}

The modified JMA Kobe earthquake ground motion used in this study is the 1995 Hyogo-ken-Nanbu (Kobe) earthquake record obtained from the Japan Meteorological Agency (JMA). In the analyses, the first 7.56 seconds that contains the peak pulses of the record of the ground motion record is used. The peak ground acceleration for $\mathrm{x}-, \mathrm{y}-$, and $\mathrm{z}$-directions are $0.60 \mathrm{~g}, 0.82 \mathrm{~g}$, and $0.34 \mathrm{~g}$ respectively, where $\mathrm{g}$ is the gravitational acceleration. The time history record is shown in Figure 4.9.

\subsubsection{Northridge Earthquake Ground Acceleration}

The Northridge ground motion record is obtained from the Pacific Earthquake Engineering Research (PEER) Strong Motion Database. The earthquake record in this study is the January 17, 1994 Northridge earthquake record obtained from the California Division of Mines and Geology (CDMG) Station 24207 recorded near the Pacoima Dam site with site condition classified as class A according to the United States Geological Survey (USGS). This earthquake has a moment magnitude of 6.7. The peak ground acceleration for $\mathrm{x}-, \mathrm{y}-$, and $\mathrm{z}$-directions are $1.585 \mathrm{~g}, 1.285 \mathrm{~g}$, and $1.229 \mathrm{~g}$ respectively, where $\mathrm{g}$ is the gravitational acceleration. The time history record for this earthquake is shown in Figure 4.10. In addition, the $\mathrm{x}^{-}, \mathrm{y}-$, and $\mathrm{z}-$ acceleration response spectra are also presented in Figure 4.11. 


\subsubsection{Kobe Earthquake Ground Acceleration}

The Kobe ground motion record is obtained from the Pacific Earthquake Engineering Research (PEER) Strong Motion Database. The earthquake record in this study is the January 16, 1995 Kobe earthquake record obtained from the Japan Meteorological Agency (JMA) Station KJMA with site condition classified as class B according to USGS. This earthquake has a moment magnitude of 6.9. The peak ground acceleration for $\mathrm{x}-, \mathrm{y}-$, and $\mathrm{z}$-directions are $0.821 \mathrm{~g}, 0.599 \mathrm{~g}$, and $0.343 \mathrm{~g}$ respectively, where $\mathrm{g}$ is the gravitational acceleration. The time history record for this earthquake is shown in Figure 4.12. In addition, the $x-, y-$, and z-acceleration response spectra are also presented in Figure 4.13.

\subsubsection{Chi-Chi Earthquake Ground Acceleration}

The Northridge ground motion record is obtained from the Pacific Earthquake Engineering Research (PEER) Strong Motion Database. The earthquake record in this study is the September 20,1999 Chi-Chi earthquake record obtained from the Central Weather Bureau (CWB) Taiwan Station CHY028 with site condition classified as class $\mathrm{C}$ according to USGS. This earthquake has a moment magnitude of 7.6. The peak ground acceleration for $x-, y$-, and z-directions are 0.821 $\mathrm{g}, 0.653 \mathrm{~g}$, and $0.337 \mathrm{~g}$ respectively, where $\mathrm{g}$ is the gravitational acceleration. The time history record for this earthquake is shown in Figure 4.14. In addition, the x-, y-, and z-acceleration response spectra are also presented in Figure 4.15. 
Table 4.1. Concrete Pier Properties of the Short-Span Bridge Model [Casarotti and Pinho, 2006]

\begin{tabular}{c|c|c|c}
\hline Parameter & Pier 2 & Pier 3 & Pier 4 \\
\hline Compressive Sirength & $3.212 \times 10^{6} \mathrm{~kg} / \mathrm{m}^{2}$ & $3.569 \times 10^{6} \mathrm{~kg} / \mathrm{m}^{2}$ & $4.375 \times 10^{6} \mathrm{~kg} / \mathrm{m}^{2}$ \\
Tensile Strength & $3.212 \times 10^{5} \mathrm{~kg} / \mathrm{m}^{2}$ & $3.569 \times 10^{5} \mathrm{~kg} / \mathrm{m}^{2}$ & $4.375 \times 10^{5} \mathrm{~kg} / \mathrm{m}^{2}$ \\
Strain at Unconfined Peak Stress & $0.002 \mathrm{~m} / \mathrm{m}$ & $0.002 \mathrm{~m} / \mathrm{m}$ & $0.002 \mathrm{~m} / \mathrm{m}$ \\
Constant Confinement Factor & 1.2 & 1.2 & 1.2 \\
Young's Modulus & $2.549 \times 10^{9} \mathrm{~kg} / \mathrm{m}^{2}$ & $2.549 \times 10^{9} \mathrm{~kg} / \mathrm{m}^{2}$ & $2.549 \times 10^{9} \mathrm{~kg} / \mathrm{m}^{2}$ \\
Shear Modulus & $1.062 \times 10^{9} \mathrm{~kg} / \mathrm{m}^{2}$ & $1.062 \times 10^{9} \mathrm{~kg} / \mathrm{m}^{2}$ & $1.062 \times 10^{9} \mathrm{~kg} / \mathrm{m}^{2}$ \\
Specific Weight & $2549.291 \mathrm{~kg} / \mathrm{m}^{3}$ & $2549.291 \mathrm{~kg} / \mathrm{m}^{3}$ & $2549.291 \mathrm{~kg} / \mathrm{m}^{3}$ \\
Separation Strain & 0.1 & 0.1 & 0.1 \\
Friction Coefficient & 0.8 & 0.8 & 0.8 \\
\hline
\end{tabular}


Table 4.2. Steel Properties of the Short-Span Bridge Model

\begin{tabular}{c|c}
\hline Properties & Steel \\
\hline Compressive Strength / Yield Stress & $3.6 \times 10^{7} \mathrm{~kg} / \mathrm{m}^{2}$ \\
Tensile Strength / Yield Stress & $3.6 \times 10^{7} \mathrm{~kg} / \mathrm{m}^{2}$ \\
Young's Modulus & $2.0389 \times 10^{10} \mathrm{~kg} / \mathrm{m}^{2}$ \\
Shear Modulus & $8.15561 \times 10^{9} \mathrm{~kg} / \mathrm{m}^{2}$ \\
Specific Weight & $7840 \mathrm{~kg} / \mathrm{m}^{3}$ \\
Separation Strain & 0.2 \\
Friction Coefficient & 0.8 \\
Post-yield Stiffness Ratio & 0.01 \\
\hline
\end{tabular}


Table 4.3. Concrete and Steel Properties of the Medium-Span Bridge Model

\begin{tabular}{c|c|c}
\hline Properties & Concrete & Steel \\
\hline Compressive Strength / Yield Stress & $3.0 \times 10^{6} \mathrm{~kg} / \mathrm{m}^{2}$ & $3.6 \times 10^{7} \mathrm{~kg} / \mathrm{m}^{2}$ \\
Tensile Strength / Yield Stress & $3.0 \times 10^{5} \mathrm{~kg} / \mathrm{m}^{2}$ & $3.6 \times 10^{7} \mathrm{~kg} / \mathrm{m}^{2}$ \\
Young's Modulus & $2.46074 \times 10^{9} \mathrm{~kg} / \mathrm{m}^{2}$ & $2.0389 \times 10^{10} \mathrm{~kg} / \mathrm{m}^{2}$ \\
Shear Modulus & $9.84297 \times 10^{8} \mathrm{~kg} / \mathrm{m}^{2}$ & $8.15561 \times 10^{9} \mathrm{~kg} / \mathrm{m}^{2}$ \\
Specific Weight & $2500 \mathrm{~kg} / \mathrm{m}^{3}$ & $7840 \mathrm{~kg} / \mathrm{m}^{3}$ \\
Separation Strain & 0.1 & 0.2 \\
Friction Coefficient & 0.8 & 0.8 \\
Post-yield Stiffness Ratio & - & 0.01 \\
\hline
\end{tabular}


Table 4.4. Summary of the Earthquake Ground Motions

\begin{tabular}{c|c|c|c|c}
\hline \multirow{2}{*}{$\begin{array}{c}\text { Earthquake } \\
\text { Name }\end{array}$} & \multicolumn{3}{|c|}{ Peak Ground Acceleration } & \multirow{2}{*}{ Duration } \\
\cline { 2 - 4 } & X-Direction & Y-Direction & Z-Direction & \\
\hline Artificial /Guedes, 1997] & - & $1.050 \mathrm{~g}$ & - & $4.00 \mathrm{~s}$ \\
Modified 1995 Kobe & $0.600 \mathrm{~g}$ & $0.820 \mathrm{~g}$ & $0.340 \mathrm{~g}$ & $7.56 \mathrm{~s}$ \\
1994 Northridge & $1.585 \mathrm{~g}$ & $1.285 \mathrm{~g}$ & $1.229 \mathrm{~g}$ & $40.00 \mathrm{~s}$ \\
1995 Kobe & $0.821 \mathrm{~g}$ & $0.599 \mathrm{~g}$ & $0.343 \mathrm{~g}$ & $48.00 \mathrm{~s}$ \\
1999 Chi-Chi & $0.821 \mathrm{~g}$ & $0.653 \mathrm{~g}$ & $0.337 \mathrm{~g}$ & $360.00 \mathrm{~s}$ \\
\hline
\end{tabular}




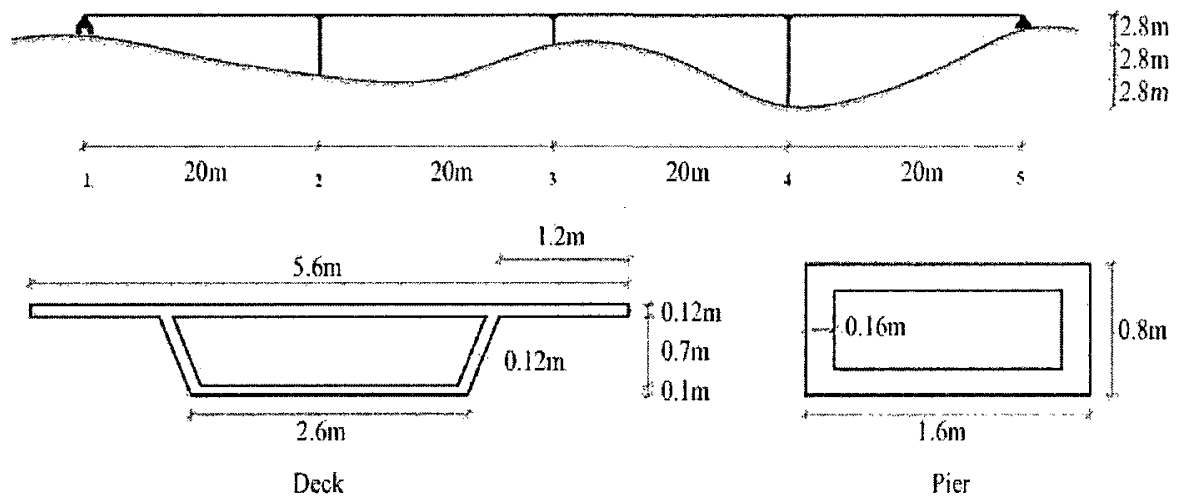

(Note: Drawings are not to scale)

Figure 4.1. Short-Span Bridge Elevation and Sections [Casarotti and Pinho, 2006] 


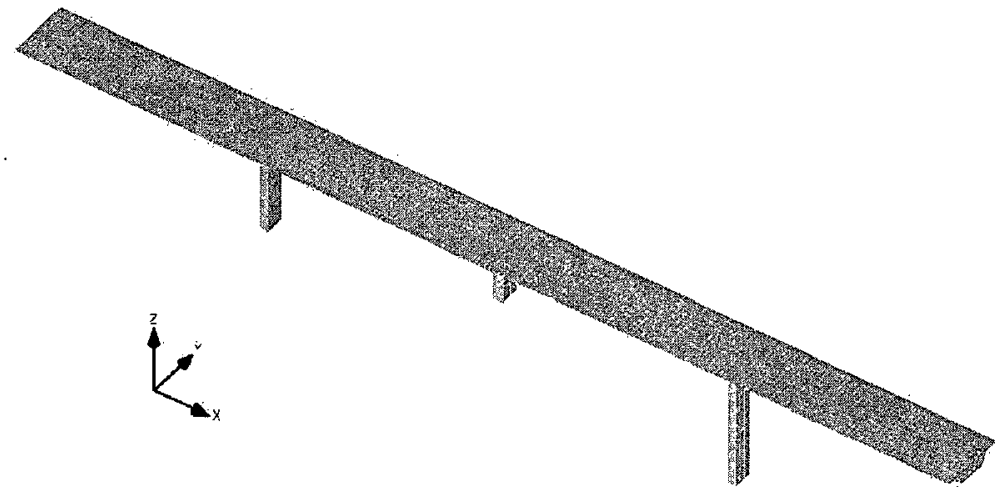

Figure 4.2. 3D View of the Short-Span Bridge Model in ELS 


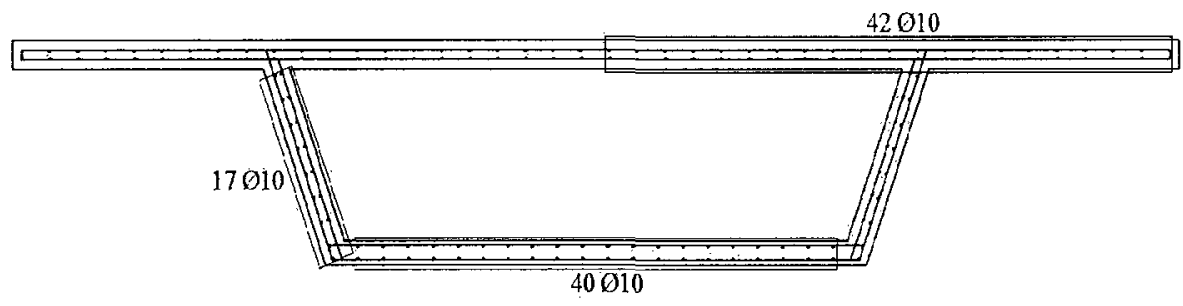

Figure 4.3. Box Girder's Longitudinal Reinforcement Details 


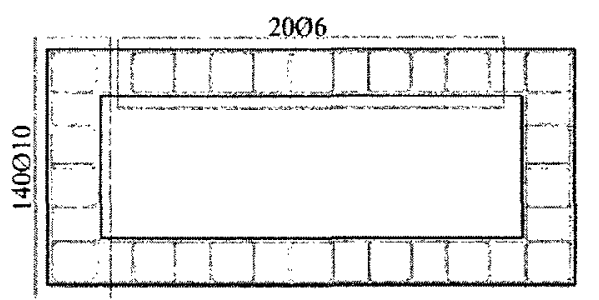

(a)

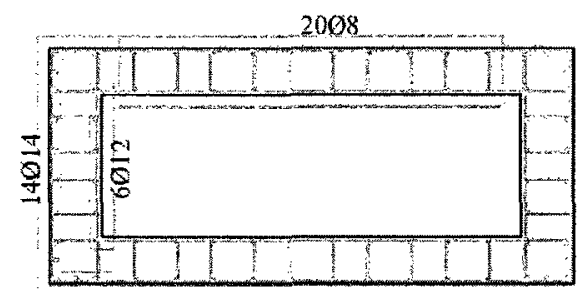

(b)

Figure 4.4. (a) Type 1 and (b) Type 2 Piers' Longitudinal Reinforcement Details [Casarotti and Pinho, 2006] 


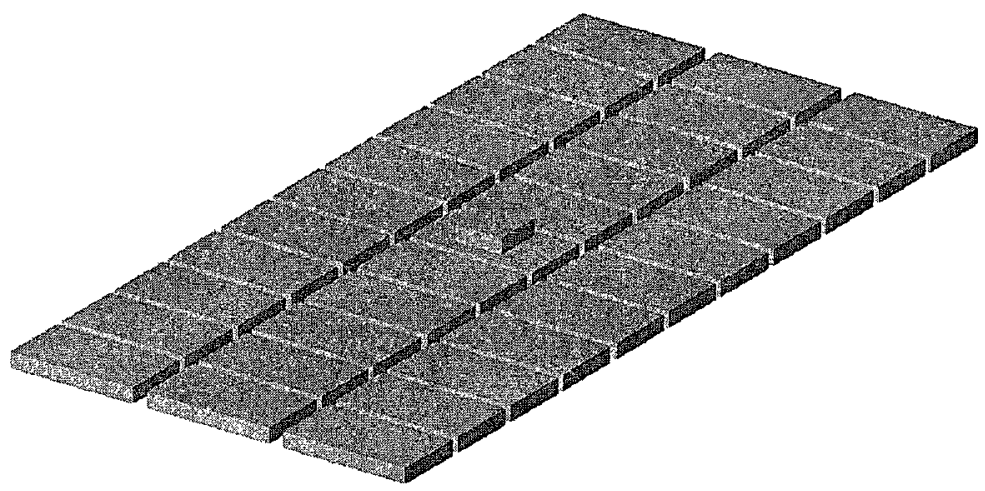

Figure 4.5. Cap on Top of the Pier to Model Pin Connection 


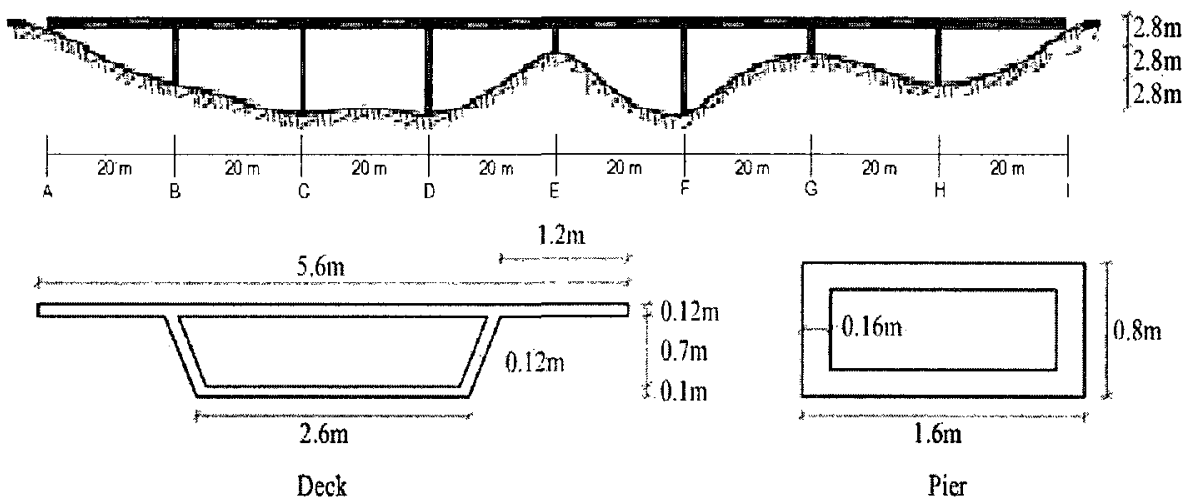

(Note: Drawings are not to scale)

Figure 4.6. Medium-Span Bridge Elevation and Sections [Casarotti and Pinho, 2006, 2007] 


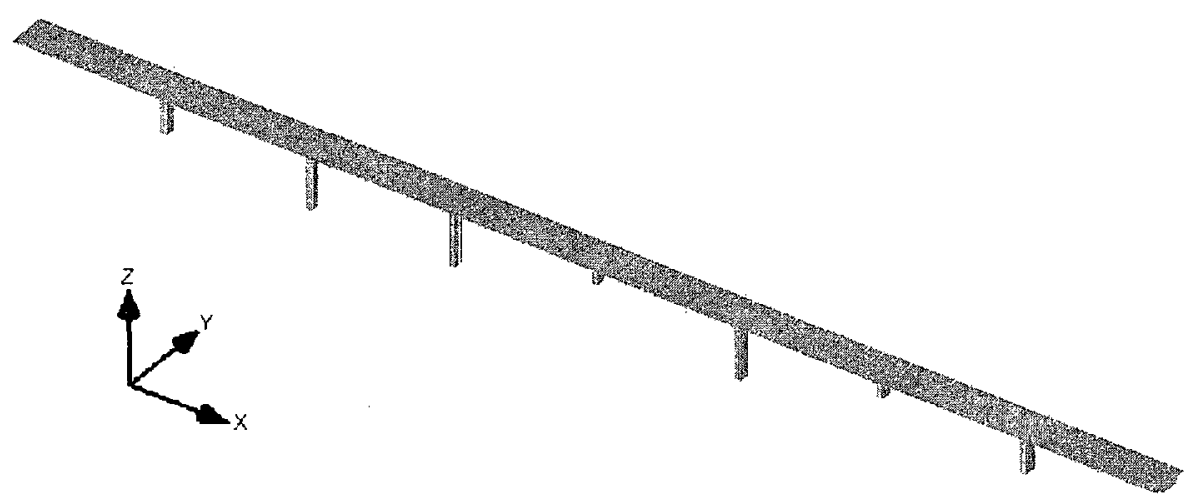

Figure 4.7. 3D View of the Medium-Span Bridge Model in ELS 


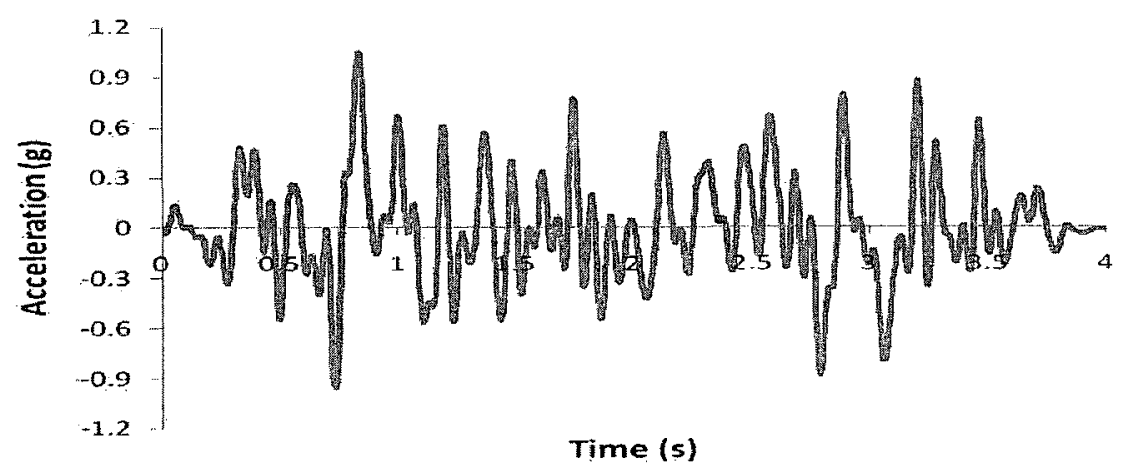

Figure 4.8. Artificial Earthquake Ground Motion from the Thesis by Guedes [1997] 


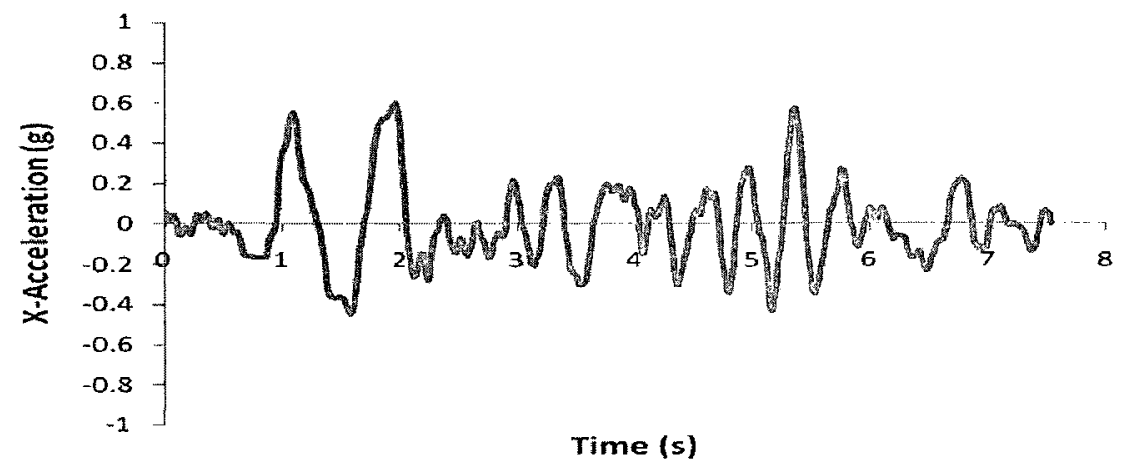

(a)

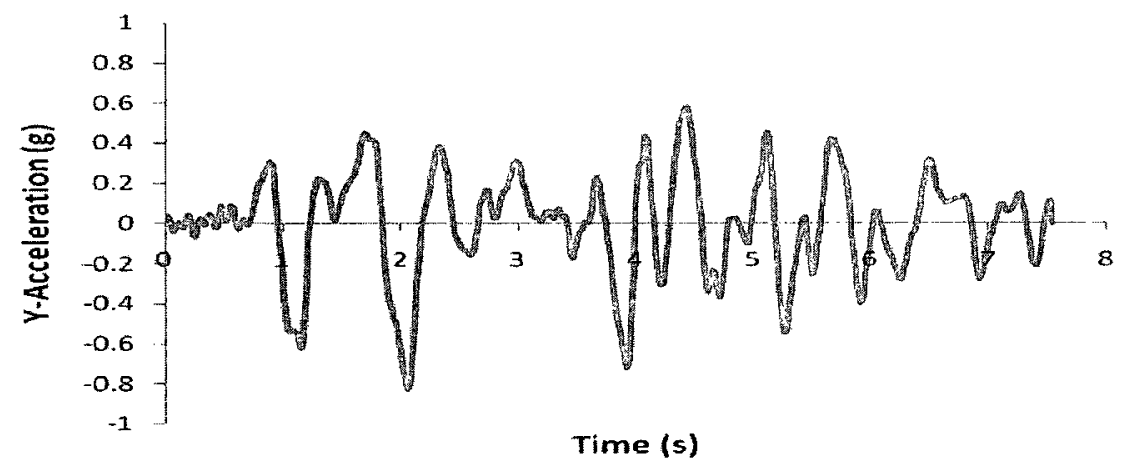

(b)

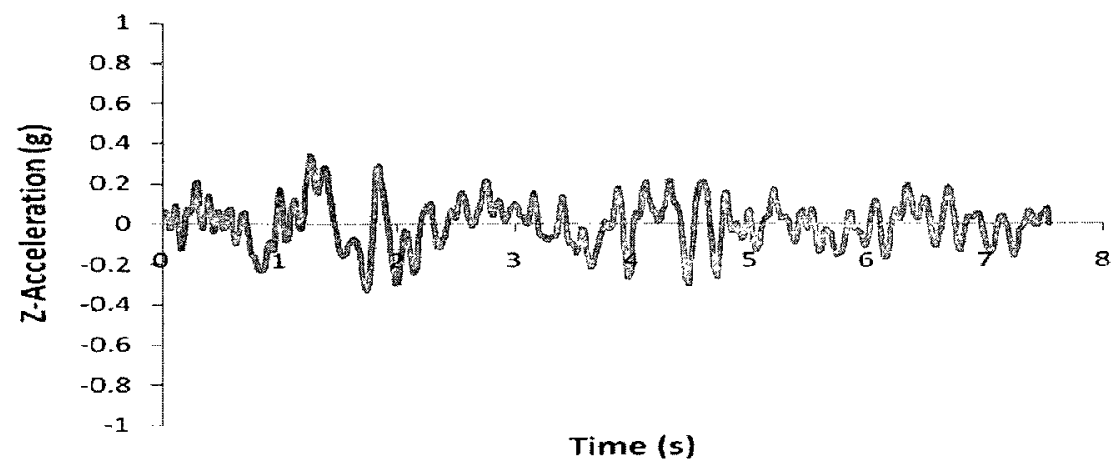

(c)

Figure 4.9. (a) X-, (b) Y-, and (c) Z-Acceleration Components of the Modified Kobe Earthquake Ground Motion 


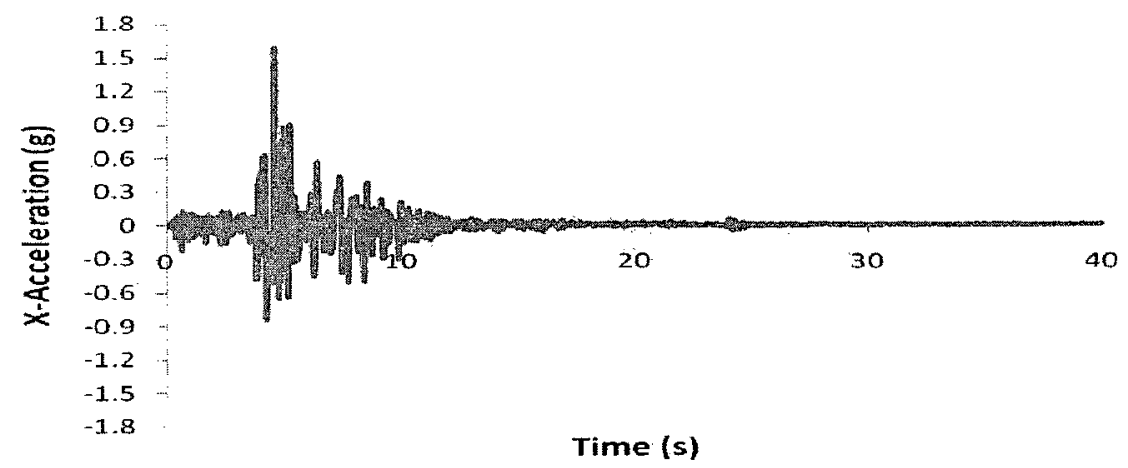

(a)

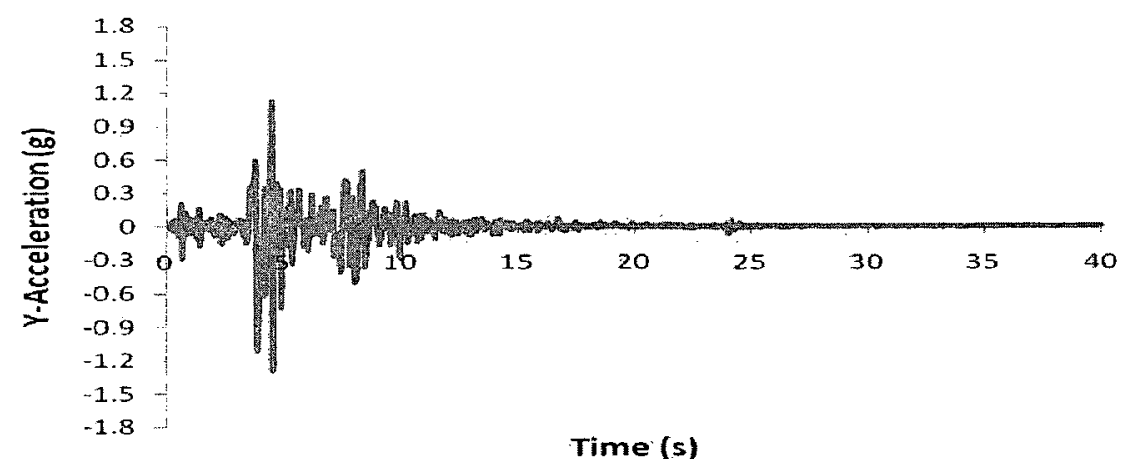

(b)

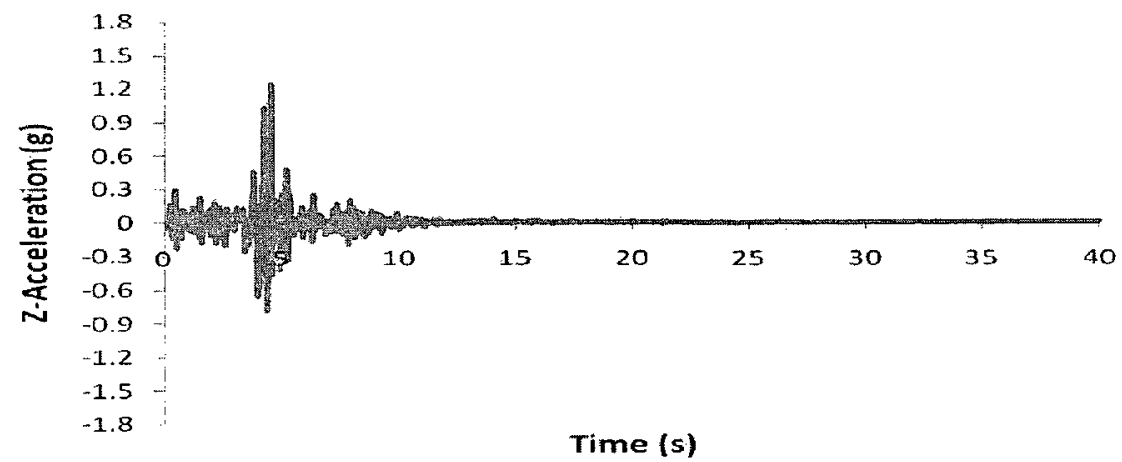

(c)

Figure 4.10. (a) X-, (b) Y-, and (c) Z-Acceleration Components of the 1994 Northridge Earthquake Ground Motion 


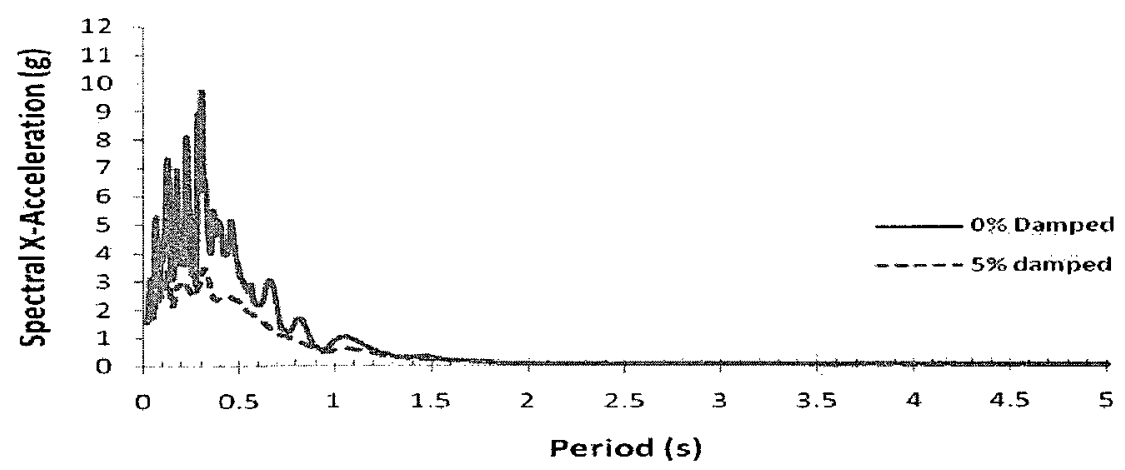

(a)

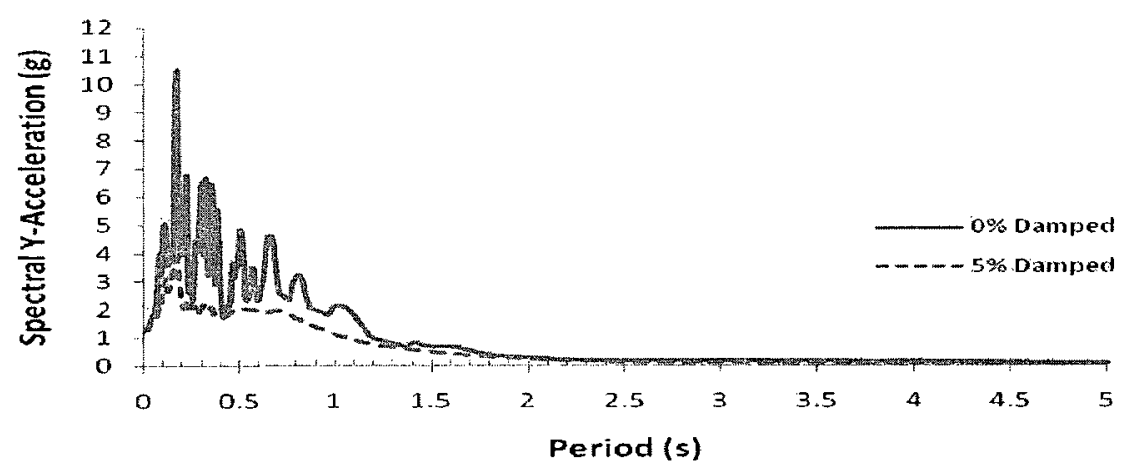

(b)

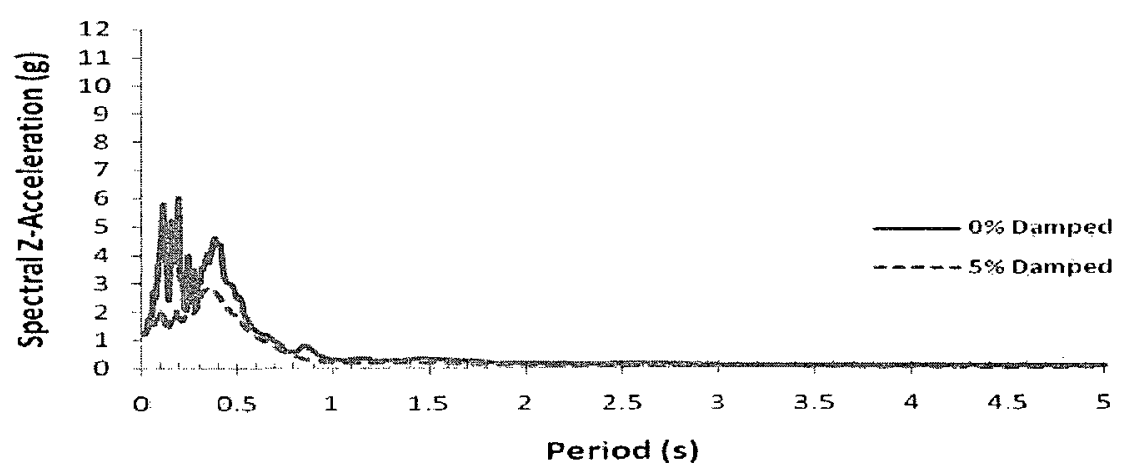

(c)

Figure 4.11. (a) X-, (b) Y-, and (c) Z-Acceleration Response Spectra of the 1994 Northridge Earthquake Ground Motion 


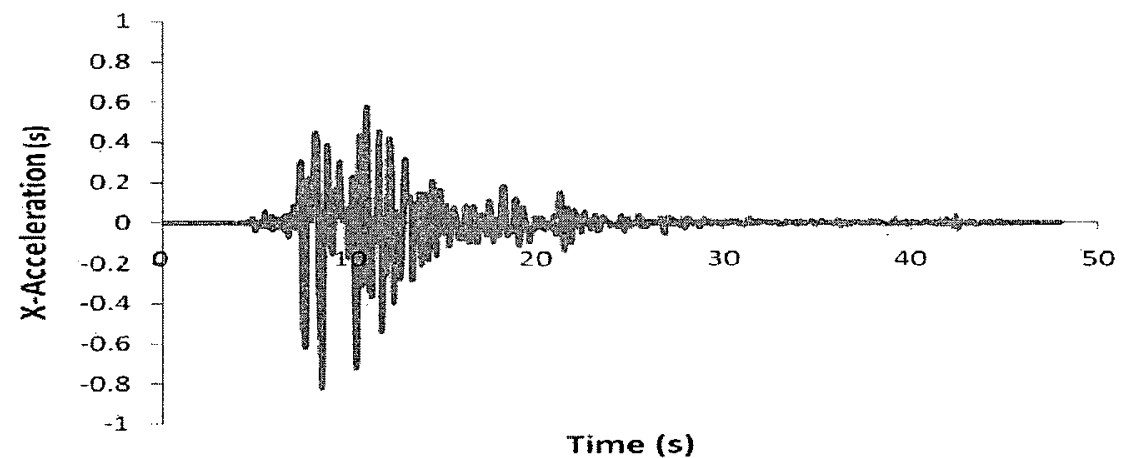

(a)

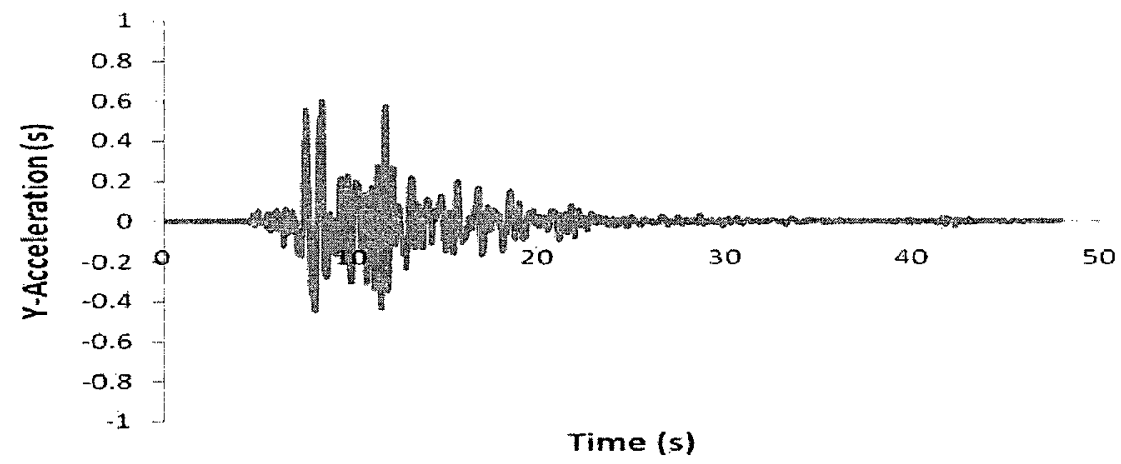

(b)

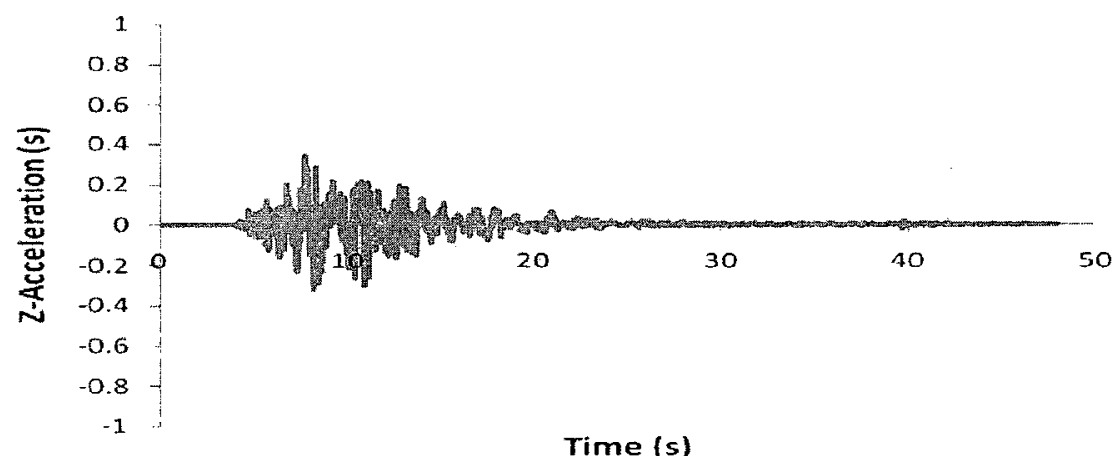

(c)

Figure 4.12. (a) X-, (b) Y-, and (c) Z-Acceleration Components of the 1995 Kobe Earthquake Ground Motion 


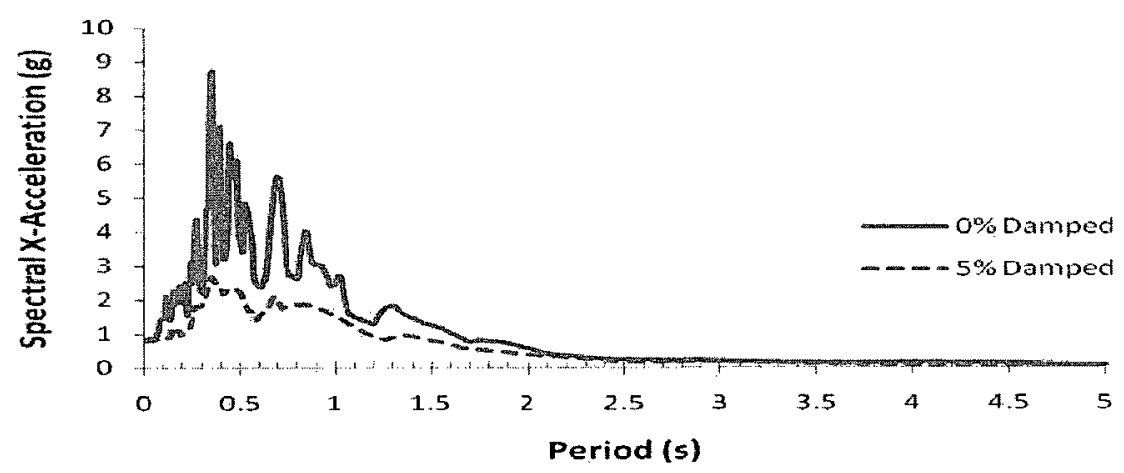

(a)

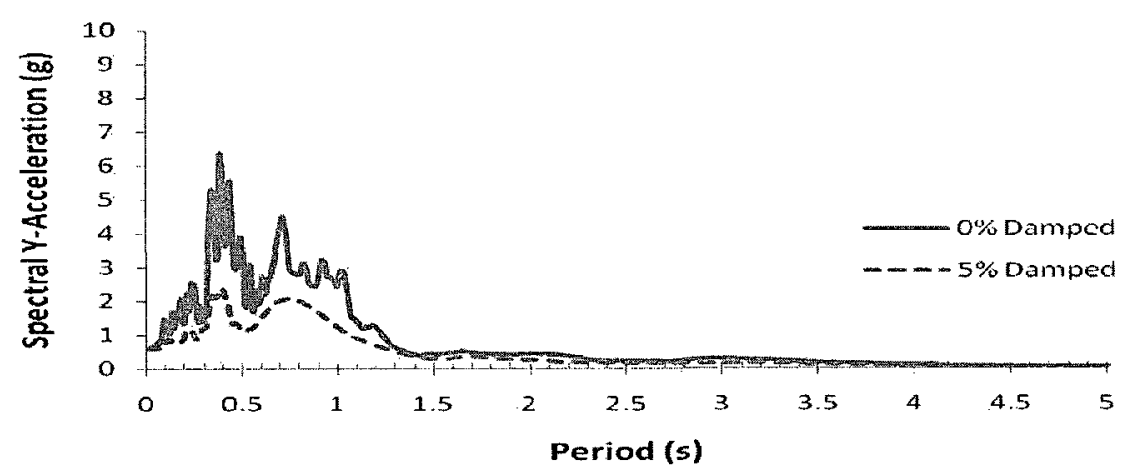

(b)

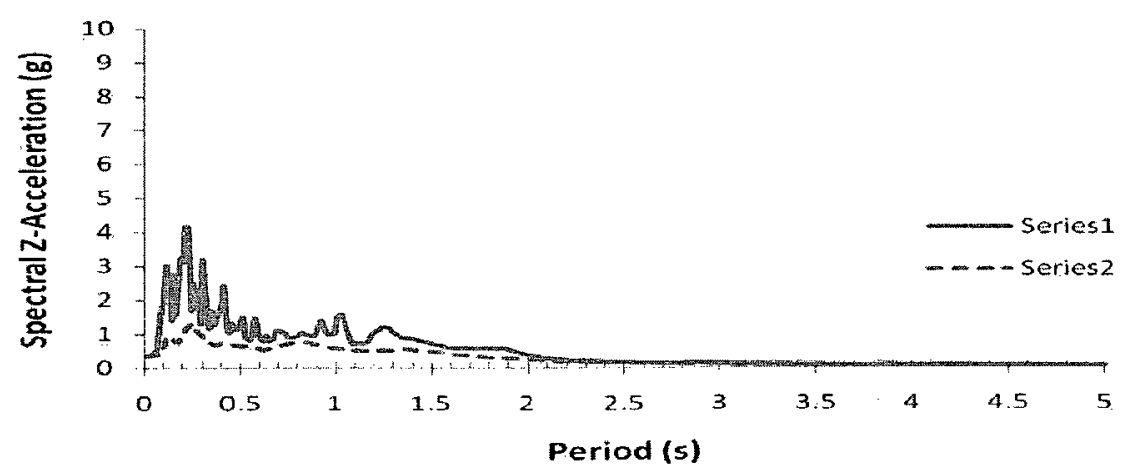

(c)

Figure 4.13. (a) X-, (b) Y-, and (c) Z-Acceleration Response Spectra of the 1995 Kobe Earthquake Ground Motion 


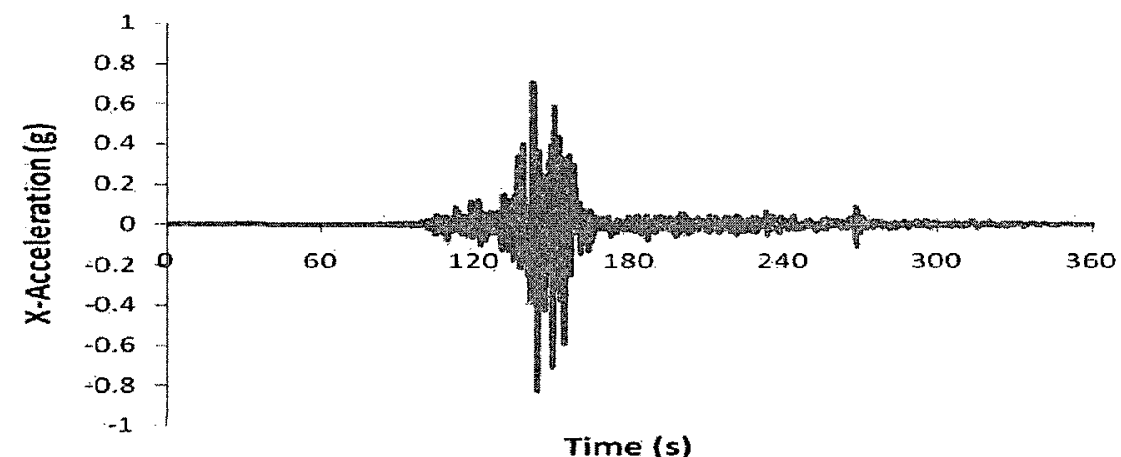

(a)

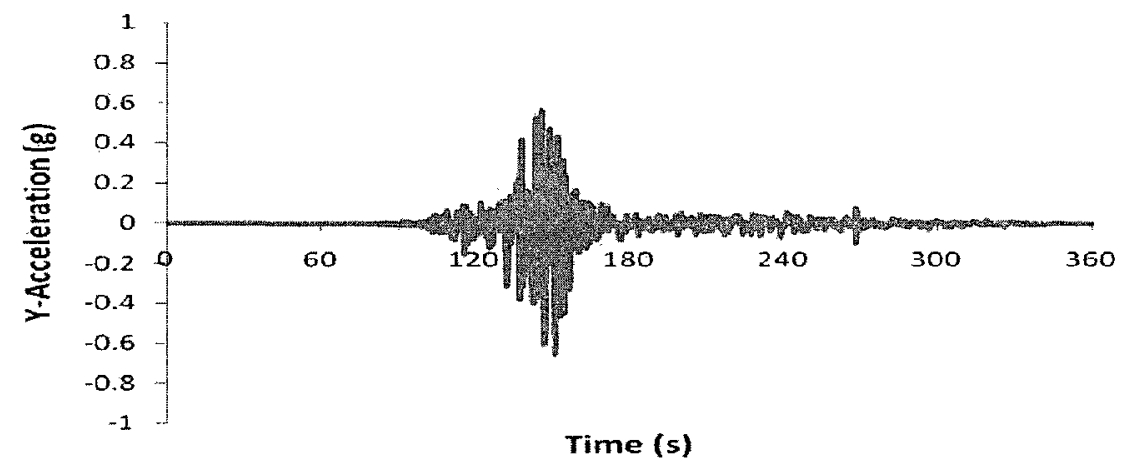

(b)

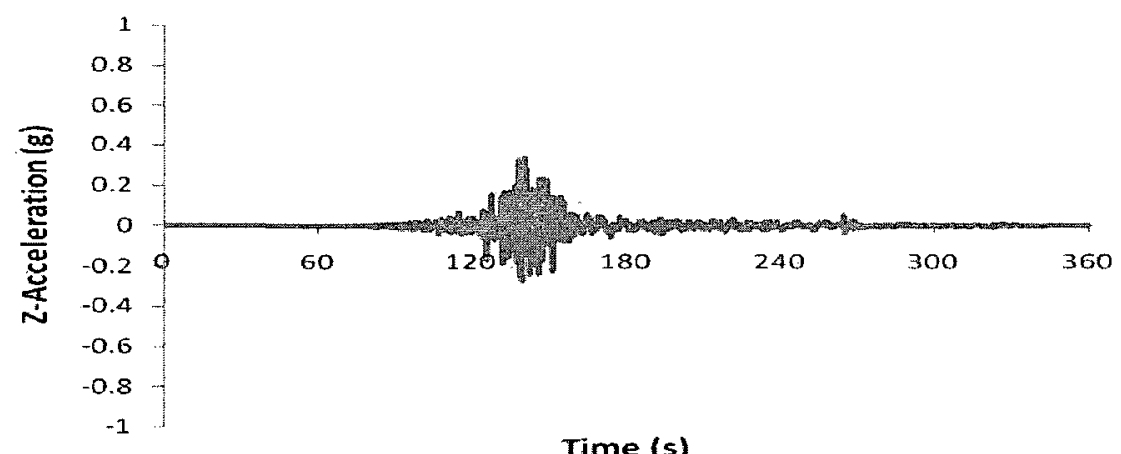

(c)

Figure 4.14. (a) X-, (b) Y-, and (c) Z-Acceleration Components of the 1999 Chi-Chi Earthquake Ground Motion 


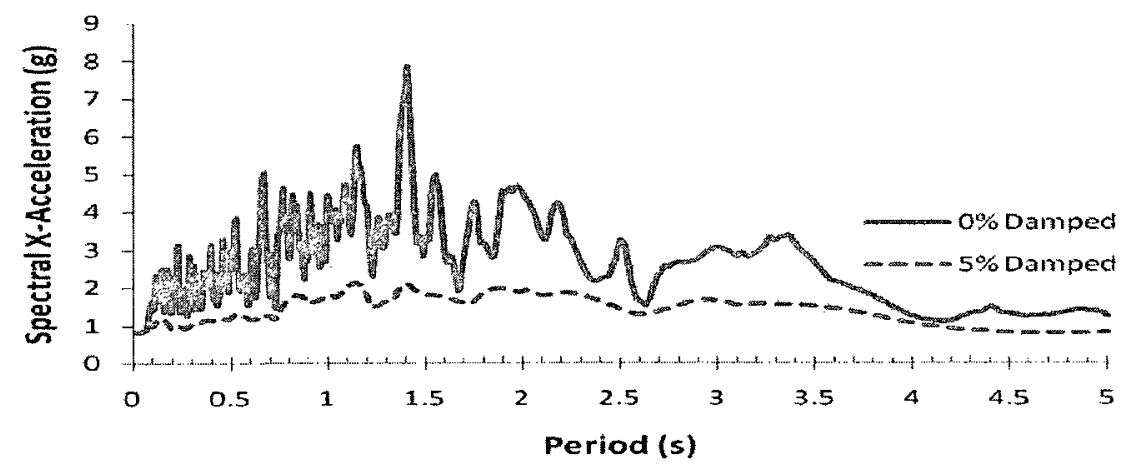

(a)

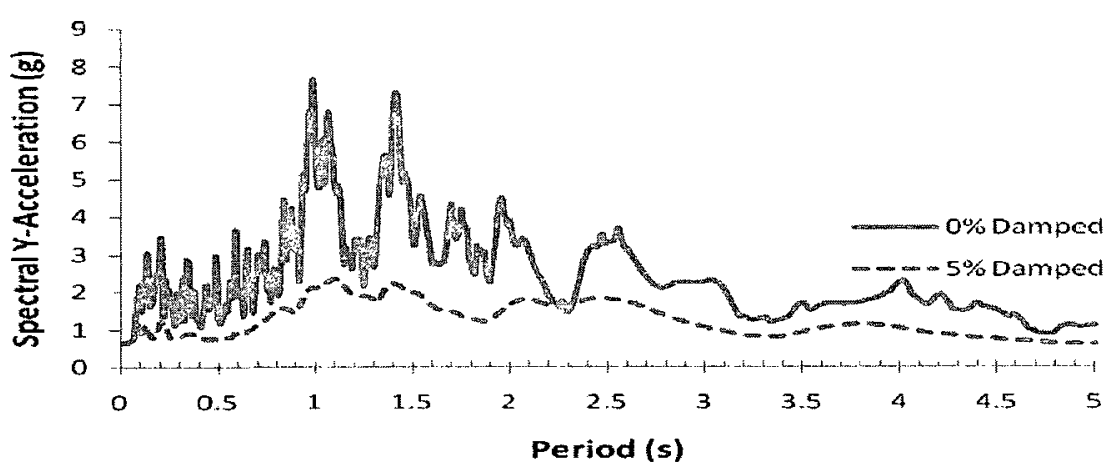

(b)

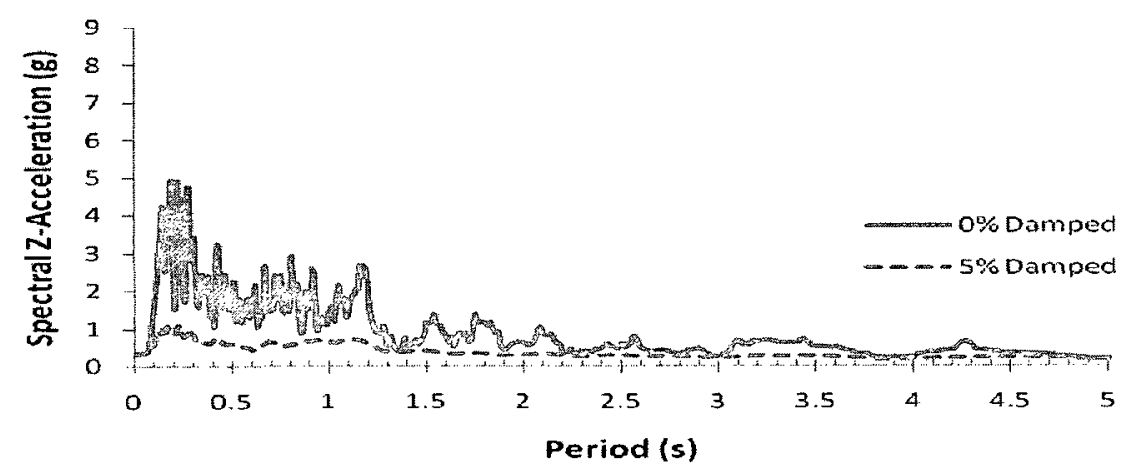

(c)

Figure 4.15. (a) X-, (b) Y-, and (c) Z-Acceleration Response Spectra of the 1999 ChiChi Earthquake Ground Motion 


\section{CHAPTER 5}

\section{Results and Discussion}

This chapter presents and discusses the numerical verification examples and the results progressive collapse analysis of the bridge models. A simple one-bay frame is analyzed to verify the validity of the AEM in linear and nonlinear analyses of structures. The results obtained by the AEM are compared to those by the FEM. In addition, the progressive collapse phenomena of bridges during earthquakes and the factors that affect progressive collapse behaviour are discussed.

\subsection{Verification Examples}

To demonstrate the validity of the AEM, two numerical example structures have been analyzed for verification purposes; one by linear time history analysis and another by nonlinear time history analysis. The ground motion used is ten times of the modified 1995 Kobe earthquake in the $\mathrm{x}$-direction as described in Section 4.2.2. The results obtained from ELS are compared to those from SAP2000 [CSI, 2009], which is based on the FEM approach. The analysis model is a one-bay reinforced concrete frame. An example to demonstrate the effect of point and distributed loads is also presented. 


\subsubsection{Frame Model}

The structure analyzed is a one-storey single bay reinforced concrete frame with a width of $5 \mathrm{~m}$ and a height of $3 \mathrm{~m}$ and assumed to be fixed at both column ends. In ELS, each column in the numerical example is divided into a mesh of $5 \times 5$ equal elements in the column cross-section and 30 equal elements along the column height. The beam is divided into a mesh of $5 \times 5$ equal elements in the beam cross-section and 10 elements along the length of the beam. In SAP2000, the columns and beam are modelled by 30 and 10 equivalent beam-column elements respectively. The damping ratio is taken to be $5 \%$. The properties of the members are presented in Table 5.1 and the 2D view of the structure is shown in Figure 5.1.

\subsubsection{Verification Results}

The time history responses of the top displacement of one of the columns and the overturning moment, shear force, and axial force at the base of one of the columns are plotted. Figure 5.2 shows the results for the linear dynamic analysis case and Figure 5.3 shows the results for nonlinear dynamic analysis case.

As can be observed from Figures 5.2 and 5.3 below, the results obtained by ELS agree with those obtained by SAP2000. The differences in the column base overturning moment, shear force, and axial force are the gravity load effect of the 
internal forces due to the self-weight of the frame which are not included in the SAP2000 results. The ELS results include the gravity load effects, whereas the SAP2000 results are due to the dynamic responses only. The axial force, shear force, and overturning moment at the base of each column due to the self-weight of the frame are calculated as $13.205 \mathrm{kN}(1346.514 \mathrm{kgf}), 1.484 \mathrm{kN}(151.371 \mathrm{kgf})$, and 1.343 kN.m (136.947 kgf.m), respectively. Moreover, the vibration periods of the structure are tabulated in Table 5.2 and the first six modes are shown in Figure 5.4. The vibration periods of the first six modes obtained from ELS and SAP2000 are similar with a maximum difference of about $7.1 \%$.

\subsubsection{Effects of Point and Distributed Loads}

As mentioned previously, the AEM procedures implemented for 3D problems do not take into account the Poisson's ratio effect. However, the analysis results show some differences between the cases of a point load and a distributed load applied to a column. These two different cases of load application are investigated. Figure 5.5 shows two cases of load position, one is a point load at the centre of the concrete column cross-section and the other is a distributed load applied on the top cross-section of the concrete column. The total applied load for each case is $9.807 \mathrm{kN}$ (1000 kgf) and the concrete column cross-section is $1 \mathrm{~m} \mathrm{x} 1 \mathrm{~m}$ and $1 \mathrm{~m}$ in length with the properties as described in Table 5.1. 
The deformations of the concrete elements in both cases are shown in Figure 5.6. It can be seen that the point load causes the springs located under the load to displace vertically and the rigid body element to translate accordingly. The deformations of the surrounding springs are then obtained from the equilibrium condition. It can also be seen that the concrete column expands slightly on its sides due to the barrelling effect of the springs and rigid bodies, as similar to the Poisson's ratio effect. On the other hand, for distributed load, the effect of compression is observed as more uniform over the loaded elements and connecting springs. Therefore, the elements of the concrete column on a cross-section have equal vertical deformations.

There are three elements that are chosen to represent the displacements in the $x-, y-$, and $z$-axes as shown in Figure 5.7. The displacement values for the elements are shown in Figure 5.8 for point load and Figure 5.9 for distributed load. As expected, displacement caused by point load is larger since the force is concentrated at one point. The negative values of the result mean that the elements are displacing along the negative global axis. The results show that the AEM procedures can take into account the effects of load positioning on the structures, regardless of the Poisson's ratio effect. 


\subsection{Seismic Progressive Collapse of Bridges with Minimum Box Girder Reinforcement}

The medium span bridge as described previously is analyzed in this study and subjected to the modified 1995 Kobe earthquake ground motion. The connection between the pier and the box girder is assumed to be rigid. The fundamental period of this bridge model is calculated as $0.245 \mathrm{~s}$. Figure 5.10 presents the progressive collapse results obtained from the software ELS which show the progression of failure and collapse of the analyzed bridge. It can be observed that cracks are formed first at the connections between the box girder and the pier. Then they propagate through the full width of the box girder soon after the joints fail. The collapse sequence results show that the AEM can accurately predict the progressive collapse behaviour of the bridge from initiation of failure until final collapse stage.

\subsubsection{Displacements}

The vertical displacement time history of the middle element of deck E-F is plotted in Figure 5.11. The graph shows that the element starts to fall at $0.4 \mathrm{~s}$ after the earthquake motion starts, and it reaches ground level at $2.1 \mathrm{~s}$ almost like a free fall motion. Figure 5.12(a) shows the displacement time history of Pier $\mathrm{F}$ along the longitudinal direction $\mathrm{x}$-axis. After the box girder collapses, the pier behaves as a cantilever column and no longer connected as an integral part of the structural system of the bridge in conjunction with the other piers. Without the lateral bracing 
constraint from the rest of the bridge system, the pier starts to displace more than it is designed for during the remaining duration of the earthquake event. During the collapse process, some parts of the pier also spall and fall. Figure 5.12(b) shows the comparison of displacement response of a falling spalled-off concrete piece from the top of the pier as captured by the AEM model and the displacement response at the top of the pier. With the reduced cross-section of the pier due to spalling of concrete, the stiffness of the pier is degraded and hence the lateral displacement of the pier increases.

\subsubsection{Internal Forces}

The piers' internal forces also experience significant jumps after one of the elements fails. Before collapse, both Piers $\mathrm{E}$ and $\mathrm{F}$ have similar motion responses. After the box girder collapses, the piers internal forces become less since they no longer bear loads from the box girder as the piers start to response independently and also bear loads from the debris caused by the falling girder elements. Figure 5.13 shows the overturning moment time history about the $y$-axis at the base of Piers $\mathrm{E}$ and $\mathrm{F}$ which show how progressive collapse behaviour affects the internal forces of the members. The shear and axial forces in the piers also experience similar effects caused by the box girder failure as shown in Figures 5.14 and 5.15. Another jump in internal forces can be observed approximately at time $2 \mathrm{~s}$. This increase is caused by an impact force when the rubble from the box girder hits the pier. 


\subsection{Parametric Study}

There are many factors that can affect the progressive collapse behaviour of structures. Some factors are related to the structure itself such as stiffness, mass, and deterioration condition of the structure. Others are related to the characteristics of the ground excitations. In this section, the factors that affect the behaviour and response of structures, which can be related to progressive collapse phenomena, are discussed.

\subsubsection{Effects of Dynamic Properties and Deterioration of the Structure}

The period of a structure is dependent on its mass and stiffness distributions. An example of the effect of period is implemented to a viaduct with a width of $5 \mathrm{~m}$ and a height of $3 \mathrm{~m}$ and assumed to be fixed at both column ends as shown in Figure 5.16. The beam, columns, and mass applied in each element of the beam of the viaduct are set to vary to obtain different values of the viaduct's fundamental period as shown in Table 5.3. The 1994 Northridge earthquake ground motion is used for this study. There are two types of beam used for this study as shown in Figure 5.17, one is with a normal reinforcement (B1) and the other is more heavily-reinforced (B2) to model elastic beam. There are also two types of column as drawn in Figure 5.18, one with eight \#9 rebars $(\mathrm{C} 1)$ and the other with twelve \#9 rebars $(\mathrm{C} 2)$ to provide higher ductility capacity. The transversal reinforcements are assumed to be the same. In ELS, each column in the numerical example is divided into a mesh of $5 \times 5$ equal 
elements in the cross-section and 30 elements along the height of the column and the beam is divided into a mesh of $5 \times 5$ equal elements in the cross-section and 10 elements along the length of the beam. The viaduct is subjected to the 1994 Northridge earthquake described previously in Section 4.2.3.

The viaduct with the least reinforcements, viaduct $\mathrm{B} 1 \mathrm{Cl}-\mathrm{A}$, does not experience any significant damage and behaves linear elastically throughout the entire time history. An interesting observation can be made when comparing the results of viaducts $\mathrm{B} 2 \mathrm{C} 2-\mathrm{B}$ and $\mathrm{B} 2 \mathrm{C} 2-\mathrm{C}$. As can be seen in Figure 5.19, viaduct B2C2-B does not have any separation of elements although it experiences minor cracking on the beam and columns surfaces. However, viaduct $\mathrm{B} 2 \mathrm{C} 2-\mathrm{C}$, as shown in Figure 5.20, shows significant damage at the base of the columns. These damages show that plastic hinges are formed and ductile behaviour of the structure is observed.

Although the earthquake excitation is applied in three directions ( $x-, y-$, and $\mathrm{z}$-axes refer to Figure 5.16), the viaduct's displacement is mostly along the $\mathrm{x}$-axis (longitudinal direction). Figure 5.21(a) shows the displacement along the $\mathrm{x}$-axis time histories for both cases obtained from the top of the columns. The results show that the response of viaduct $\mathrm{B} 2 \mathrm{C} 2-\mathrm{B}$ resembles the excitation force applied whereas the response of viaduct $\mathrm{B} 2 \mathrm{C} 2-\mathrm{C}$ tends to differ especially after damage occurs. Viaduct B2C2-C also suffers permanent damages after around $5 \mathrm{~s}$ and that permanent inelastic displacement is evident in the response even after the earthquake excitation has ended. 
The moments, shear forces, and axial forces calculated at the base of the column are presented in Figures 5.21(b), (c), and (d), respectively. The internal forces from viaduct $\mathrm{B} 2 \mathrm{C} 2-\mathrm{B}$ are lower than those from viaduct $\mathrm{B} 2 \mathrm{C} 2-\mathrm{C}$ since it is subjected to a lower inertial force as illustrated in the response spectrum. Viaduct $\mathrm{B} 2 \mathrm{C} 2-\mathrm{C}$ cannot sustain the moment and shear force demands after the columns are damaged and plastic hinges have formed. However, the columns still have the capacity to sustain gravity axial load of the structure.

When the longitudinal reinforcement in the column is reduced as in the case of viaduct $\mathrm{B} 2 \mathrm{C} 1-\mathrm{C}$, damage in the plastic hinge at the base of the column is more severe and it causes the column to collapse as can be observed in Figure 5.22. The column does not have sufficient ductility capacity to resist the inelastic deformation demand and fails in bending. Once the column, which is the vertical load bearing component, fails, the beam no longer has the vertical support and starts having excessive deformation that further leads to the collapse of the entire system. A brittle failure can be observed in the case of viaduct $\mathrm{B} 1 \mathrm{C} 2-\mathrm{C}$ with less reinforcement in the beam as shown in Figure 5.23. The damage starts in the plastic hinge area of the column but move to the beam-column connection where the separation occurs. The beam then begins to have large deflection at the point where maximum moment caused by the combined lateral and gravity loads occurs. After that the beam collapses and drags the columns with the final collapse mechanism developed.

Comparison of the top displacements and internal forces for viaducts with mass of $150 \mathrm{~kg}$ applied in each element of the beam (B1C1-C, B1C2-C, B2C1-C, and 
$\mathrm{B} 2 \mathrm{C} 2-\mathrm{C}$ ) are presented in Figure 5.24. It can be seen that only viaduct $\mathrm{B} 2 \mathrm{C} 2-\mathrm{C}$ does not collapse during the earthquake shaking. Moreover, the internal forces for the columns before the girder completely collapses may experience significant jump caused by impact force from falling debris.

\subsubsection{Effects of Earthquake Ground Motion}

To demonstrate the effects caused by different ground motions, the shortspan bridge model is used. The earthquake ground motions applied are the 1994 Northridge, the 1995 Kobe, and the 1999 Chi-Chi earthquakes presented previously in Sections 4.2.3 to 4.2.5. The characteristics of the earthquake records, such as the peak acceleration, frequency content, intensity, and duration of strong shaking can significantly affect the response behaviour of the structures.

The progression of the structural failure and collapse of the analyzed bridge due to the 1994 Northridge, the 1995 Kobe, and the 1999 Chi-Chi earthquakes are captured in Figures 5.25 to 5.27, respectively. From observation, it can be noticed that the 1999 Chi-Chi earthquake caused the most damage to the bridge structure. Due to this earthquake, the box girder starts to fail early in the response and that actuates the short pier to bear more loads beyond its capacity and thus leads to its subsequent failure. The short pier collapses in flexure since it attracts the most seismic force. This short pier collapse also greatly reduces the load resistant capacity of the bridge resulting in the ramification effect of load shifts in the whole system 
which finally leads to the tall pier collapses. The collapse of the pier can also happen as a result of impact force from the failure of the box girder as can be observed in the collapse of the tall pier due to the 1995 Kobe earthquake.

The displacement time histories along the $\mathrm{x}$-axis at the top of the piers caused by the 1994 Northridge, the 1995 Kobe, and the 1999 Chi-Chi earthquakes are shown in Figures 5.28 to 5.30, respectively. The collapse of the girder results in the pier losing its lateral bracing support, then it displaces more from the dragging action of the falling girder before it is completely separated from the pier during the response. The pier can also experience permanent deformation caused by the tilting and dislocation of the pier. Figure 5.31 shows an example of dislocation and cracks in the pier due to the 1995 Kobe earthquake excitation, where a part of the short pier is no longer completely intact at the base. The internal forces of the piers due to the three earthquakes are plotted in Figures 5.32 to 5.34, respectively. It can be seen that the internal forces have the same patterns as the displacements. The jumps in the internal forces occur when the abutments fail and the loads from the box girder are transferred fully to the piers and when there are impact forces from the debris.

\subsection{Evaluation on the Assumption of Elastic Superstructure Model}

Analysis of bridges using the FEM usually assumes the superstructure to be elastic to simplify the analysis and reduce the computational time. This assumption is 
based on commonly accepted seismic design assumption and bridge damage observations in previous major earthquakes that the bridge superstructure still remains elastic before column piers suffer damage from plastic hinging.

In order to evaluate the validity of the elastic bridge superstructure assumption, two analyses using ELS have been carried out. The first analysis assumes the box girder to be elastic and the second analysis considers inelastic behaviour in the bridge box girder. The short-span bridge model is used for this purpose and the bridge is subjected to the artificial earthquake ground motion obtained from Guedes [1997]. The analyses results are presented and compared in the following sections.

\subsubsection{Short-Span Bridge Model}

The linear dynamic properties of the short-span bridge model obtained from ELS eigenvalue analysis are compared to those in the literature. The first three modal periods and shapes for the short-span bridge model from the literature are presented in Table 5.4. In ELS, the nonlinear dynamic properties are dependent on the support modelling. Figure 5.35 illustrates three different cases for abutment modelling in ELS. Case Al is where the bridge bearing support at the abutment is assumed to be placed along the soffit of the box girder, case $\mathrm{A} 2$ is where the bearing is assumed to be placed at the middle supporting the soffit of the box girder, and case $A 3$ is where the bearings are assumed to be placed on two locations supporting the soffit of the box girder. 
The vibration modes determined from the analysis is the rocking mode of the box girder as shown in Figure 5.36. For the first three lateral modes, the results obtained from ELS as shown in Table 5.5 give higher periods compared to results from the FEM fibre-model analysis or from the experimental test [Casarotti and Pinho, 2006]. The main source of these discrepancies is the element discretization since there is not enough data to compare to the FEM model used in the literature. It is also noted that modelling in ELS, which is based on the AEM, needs more details such as reinforcements and connection or support models that are not available in the literature. More details are needed since it uses 3D elements as compared to $1 \mathrm{D}$ equivalent frame elements commonly used in the FEM analyses. For example, the modelling to release the moment at the girder-pier connections in ELS has to use a transition elements but in the FEM this can be done by assigning releases at the end of the frame element. Nevertheless, the results obtained from ELS are still acceptable.

\subsubsection{Results for Elastic Superstructure Model}

The results for elastic superstructure model obtained from ELS are compared to those obtained from an experimental hybrid test conducted at the laboratory at the Joint Research Centre of Ispra and numerical results from fibre-model analyses [Casarotti and Pinho, 2006]. The earthquake record used for the analyses is the artificial earthquake record from the thesis by Guedes [1997] as presented in Section 4.2.1 applied in the transversal direction of the bridge (global y-axis direction). 
The displacement time histories for the short pier (Pier 3), medium pier (Pier 2), and tall pier (Pier 4) are shown in Figures 5.37(a) to 5.37(c), respectively. The analysis results from ELS show good matches with those obtained from the hybrid test. In some cases the analysis results from ELS are better than the results obtained from the fibre-model analyses in capturing the maximum displacements of the piers. However, the analysis results and the motion phases from ELS diverge by a small amount after reaching the peaks at about $3.2 \mathrm{~s}$. These are caused by the effect of strength and stiffness deteriorations since the area around the connections between the pier and the girders experience cracking and therefore affect the response of the structure. In the FEM analysis, this phenomenon of deterioration caused by cracks or element spallings is not captured in the previous study. The experimental hybrid test also does not take into account this deterioration effect since the box girder is modelled as linear elastic members in computer simulation. Another source of discrepancy can be attributed to the different material models used in ELS and fibremodel analyses.

Figures 5.38(a) to 5.38(c) show the comparison of top shear forces obtained from ELS, fibre-model analysis, and experimental results for short, medium, and tall piers, respectively. The shear forces obtained from the ELS are similar to those obtained from the fibre-model analyses and are very close to the results from the experimental hybrid test. The effect of strength and stiffness deteriorations is also observable after the peak responses at about $3.2 \mathrm{~s}$. The other internal forces cannot be compared since the results are not presented in the literature. 


\subsubsection{Results for Regular Superstructure Model}

Figure 5.39 shows the progression of collapse of the short-span bridge model. It can be seen that collapse initiates from the bridge abutments and then propagates to the middle spans. The abutment failure causes the short pier to be overstressed and when the girder falls it also drags the short pier to tip over. The other piers also suffer damages caused by cracks around the plastic hinge areas. This finding clearly shows the behaviour of the box girder contributes significantly to the collapse of the bridge system. The box girder spans fail at the pier supports leading to collapse of the spans.

The displacement time histories of the piers from ELS analysis with regular superstructure model compared to the elastic superstructure model are shown in Figures 5.40. It can be observed that the peak displacements of the piers before collapse of the girder using the regular superstructure model give slightly higher values compared to those calculated with the elastic superstructure model because of nonlinear behaviour of the girder. The peak displacements after the girder falls are lower since the mass participating in the dynamic response of the piers is reduced without the contributing mass from the supported girder as they behave independently as cantilevers columns.

Shear forces from the analyses by ELS with the regular superstructure model compared to those with the elastic superstructure model are shown in Figure 5.41. The shear forces also exhibit similar behaviour as the displacements where the local 
peaks from the regular superstructure model show higher values compared to those from the elastic superstructure model. The period of the responses also shifts since the piers experience damages earlier resulting in changes to the responses of the piers.

\subsection{Discussion}

The analysis results show that seismic progressive collapse phenomenon is global damage behaviour of the structure. This phenomenon is different if compared to the collapse caused by blast loads where the initiation of failure is caused by a local element removal or loss of vertical load bearing structural element. During earthquakes, part of a structure starts to fail causing redistribution of load to the remainder of the structural system and subsequent failure and development of collapse mechanisms. Analysis procedures based on the approach of element removal are not adequate to capture the sequence of failure events and propagation of damage in appropriate seismic progressive collapse of structures. An analysis tool that can follow the entire history of inelastic behaviour of structures from initiation of failure, spread of damage, element separation, impact inertial load effect of falling debris, to simulation of collapse mechanisms is necessary. The AEM and the software ELS give good results in simulation of seismic progressive collapse behaviour of structures.

The response of structures during the collapse process is highly nonlinear and influenced by the response from impact force of falling debris. There are drastic 
changes in mass, strength, and stiffness properties of the structure during the progressive collapse process. The results show that sufficient ductility capacity in bridge columns or piers helps to ensure ductile behaviour and higher deformation capability of the structure that leads to better performance against seismic progressive collapse. From the results it can also be observed that in many cases although the pier has suffered damages and permanent deformation it can still effectively carry the axial compressive loads from the bridge superstructure and its own self-weight. Damage reduces the pier shear capacity and lateral load resistance of the bridge structural system.

Structural deteriorations in old bridges may lead to reduction in the stiffness and strength properties of the structures, which can have significant impact to the behaviour and earthquake resistance capacity of the systems. The discrete element modelling approach of the AEM can capture the influence and significance of structural deteriorated conditions by using appropriate material properties and material models in the analysis.

In comparing the effects of different earthquake ground motions on the progressive collapse behaviour, it is noted that the 1994 Northridge earthquake excitation with the highest peak ground acceleration causes no pier to collapse but the 1999 Chi-Chi earthquake excitation causes significant collapse or failures of the entire bridge structures including the piers. The response spectrum of the 1999 ChiChi earthquake ground motion show that its frequency content has higher energy in longer period range compared to the 1994 Northridge earthquake. Since the bridge 
softens due to strength or stiffness deteriorations after structural members start to fail and suffer damage, the period shifts in the structure to longer due to its softening and makes it more vulnerable to more severe earthquake damage.

Progressive collapse phenomena should be considered in the seismic design of structures to increase safety. Seismic progressive collapse simulations can help structural engineers to better understand the design objective in performance-based seismic design of structures, including a more comprehensive approach in devising effective retrofit strategies for old deficient bridges by considering the pattern and severity of the potential damage in the structure. The girder collapse does not necessarily mean worse damage to the structure. Considering the recent development of rapid bridge deck or girder replacement technology, one seismic design approach of bridges against catastrophic seismic events is possibly to design strong and ductile columns, while allowing girder or deck span to fail with the expectation that the downtime of the structure can be minimized. The consideration of progressive collapse behaviour of bridges can have an impact on reducing the post-earthquake economic loss and disruptions. 
Table 5.1. Properties of the Verification Model

\begin{tabular}{c|c}
\hline Concrete Compressive Strength & $3.51535 \times 10^{6} \mathrm{~kg} / \mathrm{m}^{2}$ \\
Concrete Tensile Strength & $1.75767 \times 10^{5} \mathrm{~kg} / \mathrm{m}^{2}$ \\
Young's Modulus & $2.834 \times 10^{9} \mathrm{~kg} / \mathrm{m}^{2}$ \\
Shear Modulus & $1.181 \times 10^{9} \mathrm{~kg} / \mathrm{m}^{2}$ \\
Specific Weight & $2402.77 \mathrm{~kg} / \mathrm{m}^{3}$ \\
\hline
\end{tabular}


Table 5.2. Comparison of Vibration Periods

\begin{tabular}{c|cccccc}
\hline Mode Number & \multicolumn{1}{|c}{ J } & 2 & 3 & 4 & 5 & 6 \\
\hline Period - ELS (s) & 0.07537 & 0.02878 & 0.01076 & 0.00993 & 0.00807 & 0.00513 \\
Period -SAP2000 (s) & 0.07736 & 0.03098 & 0.01132 & 0.01010 & 0.00829 & 0.00526 \\
\hline Difference (\%) & -2.57 & -7.10 & -4.95 & -1.68 & -2.65 & -2.47 \\
\hline
\end{tabular}


Table 5.3. Comparison of Viaduct Fundamental Periods from Different Combinations

\begin{tabular}{|c|c|c|c|c|}
\hline Beam & Column & $\operatorname{Mass}^{*}(\mathrm{~kg})$ & Period (s) & Label \\
\hline \multirow{6}{*}{ B1 } & \multirow{3}{*}{$\mathrm{Cl}$} & 0 & 0.047 & B1C1-A \\
\hline & & 50 & 0.125 & $\mathrm{~B} 1 \mathrm{C} 1-\mathrm{B}$ \\
\hline & & 150 & 0.243 & $\mathrm{~B} 1 \mathrm{C} 1-\mathrm{C}$ \\
\hline & \multirow{3}{*}{$\mathrm{C} 2$} & 0 & 0.046 & $\mathrm{~B} 1 \mathrm{C} 2-\mathrm{A}$ \\
\hline & & 50 & 0.121 & $\mathrm{~B} 1 \mathrm{C} 2-\mathrm{B}$ \\
\hline & & 150 & 0.226 & $\mathrm{~B} 1 \mathrm{C} 2-\mathrm{C}$ \\
\hline \multirow{6}{*}{ B2 } & \multirow{3}{*}{$\mathrm{Cl}$} & 0 & 0.046 & B2C1-A \\
\hline & & 50 & 0.122 & $\mathrm{~B} 2 \mathrm{C} 1-\mathrm{B}$ \\
\hline & & 150 & 0.226 & $\mathrm{~B} 2 \mathrm{C} 1-\mathrm{C}$ \\
\hline & \multirow{3}{*}{$\mathrm{C} 2$} & 0 & 0.045 & $\mathrm{~B} 2 \mathrm{C} 2-\mathrm{A}$ \\
\hline & & 50 & 0.117 & $\mathrm{~B} 2 \mathrm{C} 2-\mathrm{B}$ \\
\hline & & 150 & 0.212 & $\mathrm{~B} 2 \mathrm{C} 2-\mathrm{C}$ \\
\hline
\end{tabular}

Note: $\left.{ }^{*}\right)$ Mass is applied in each element of the beam 
Table 5.4. First Three Modal Periods and Shapes in the Transversal Direction of the Box Girder from the Literature [Casarotti and Pinho, 2006]

\begin{tabular}{c|ccc}
\hline $\begin{array}{c}\text { Mode } \\
\text { Number }\end{array}$ & $\begin{array}{c}\text { Experimental } \\
\text { Period (s) }\end{array}$ & $\begin{array}{c}\text { Casarotti-Pinho } \\
\text { Period (s) }\end{array}$ \\
\hline 1 & 0.183 & 0.183 \\
\hline & 0.146 & 0.148 \\
\hline
\end{tabular}


Table 5.5. First Three Modal Periods and Shapes in the Transversal Direction of the Box Girder from ELS

\begin{tabular}{|c|c|c|c|c|}
\hline $\begin{array}{l}\text { Mode } \\
\text { Number }\end{array}$ & $\begin{array}{l}\text { A1 Period } \\
\text { (s) }\end{array}$ & $\begin{array}{c}\text { A2 Period } \\
(s)\end{array}$ & $\begin{array}{l}\text { A3 Period } \\
\text { (s) }\end{array}$ & Modal Shapes \\
\hline$I$ & 0.192 & 0.271 & 0.228 & \\
\hline 2 & 0.138 & 0.146 & 0.138 & \\
\hline 3 & 0.123 & 0.135 & 0.131 & \\
\hline
\end{tabular}




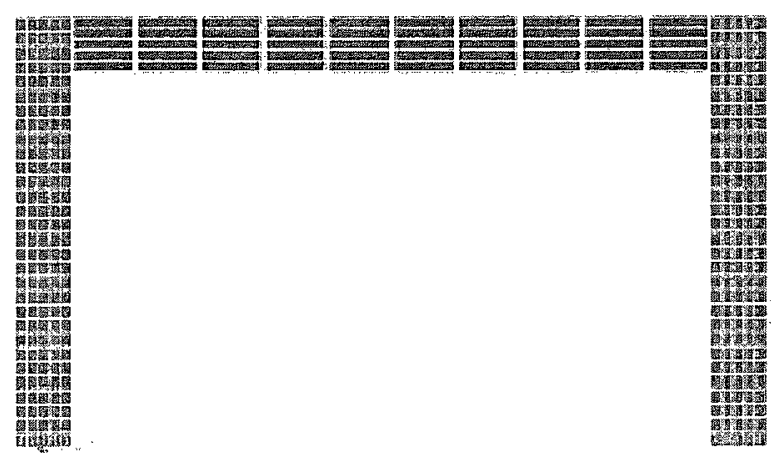

(a)

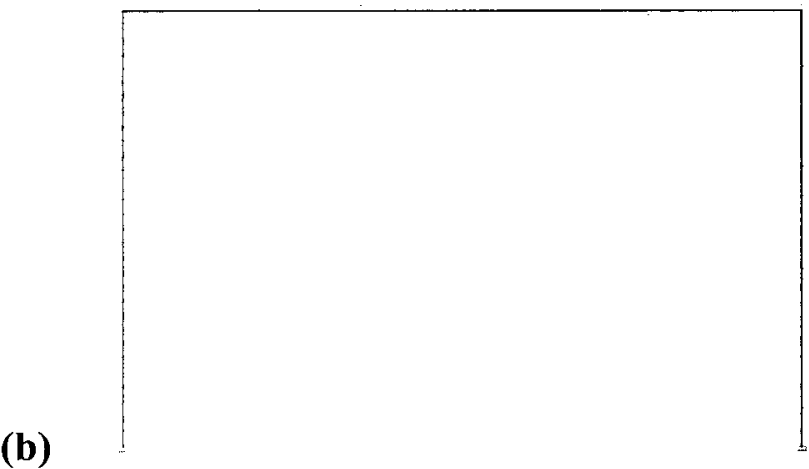

Figure 5.1. 2D View of Verification Model in (a) ELS and (b) SAP2000 


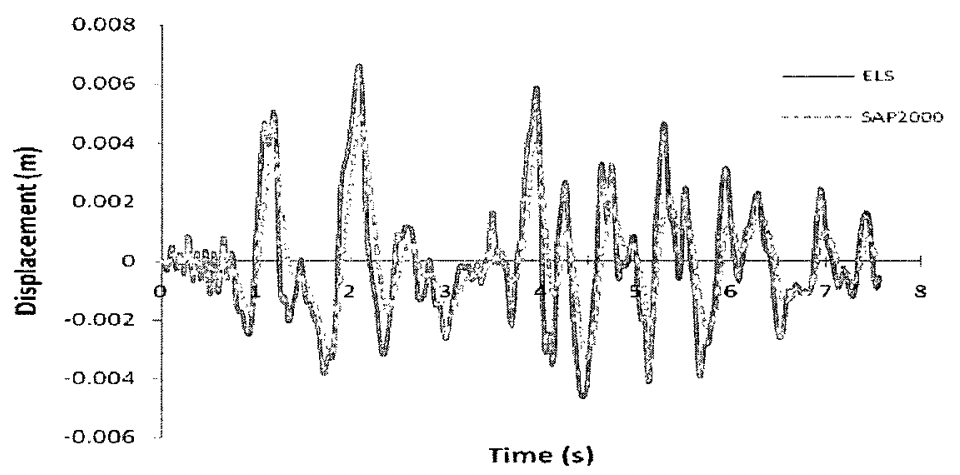

(a)

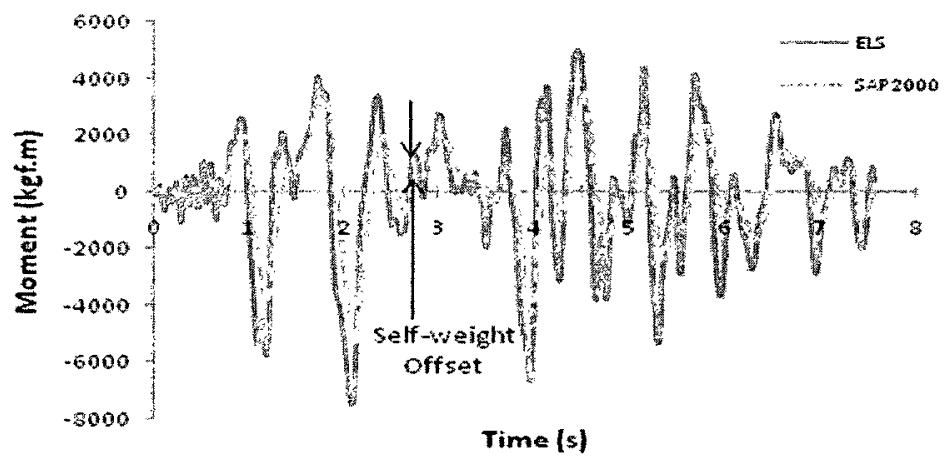

(b)

Figure 5.2. Linear Analyses Verification Results: (a) Displacement, (b) Moment, (c) Shear Force, (d) Axial Force 


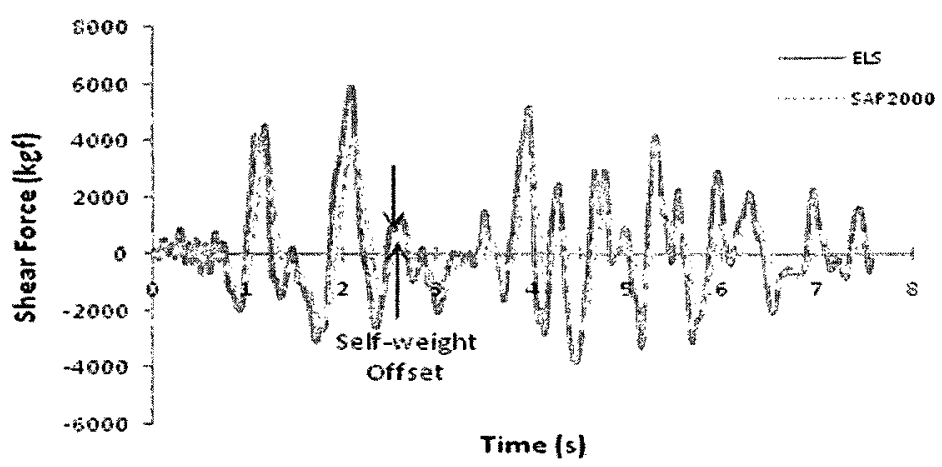

(c)

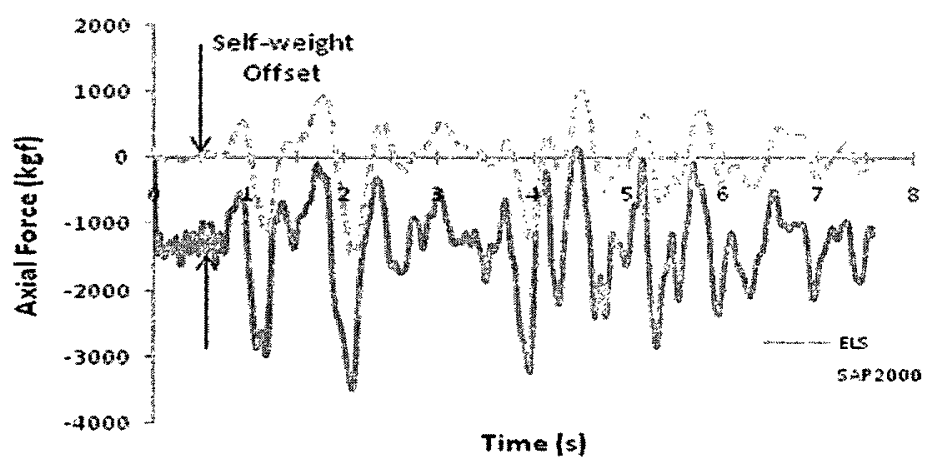

(d)

Figure 5.2. (cont'd) Linear Analyses Verification Results: (a) Displacement, (b) Moment, (c) Shear Force, (d) Axial Force 


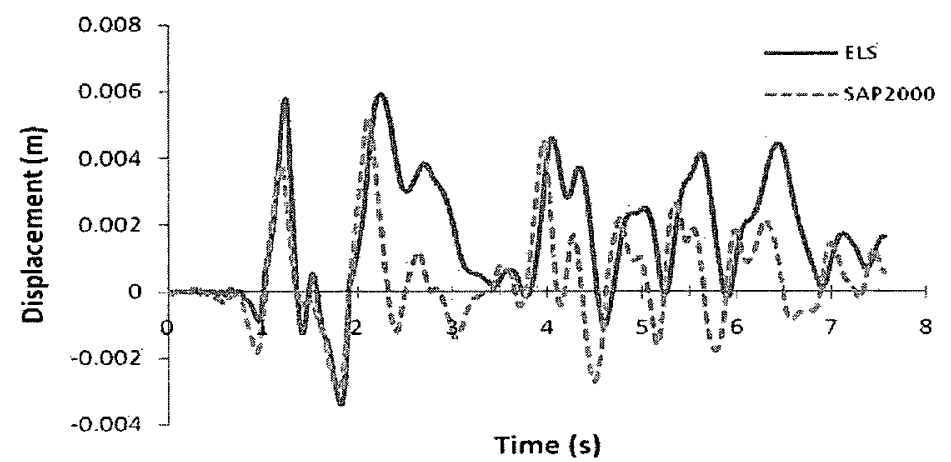

(a)

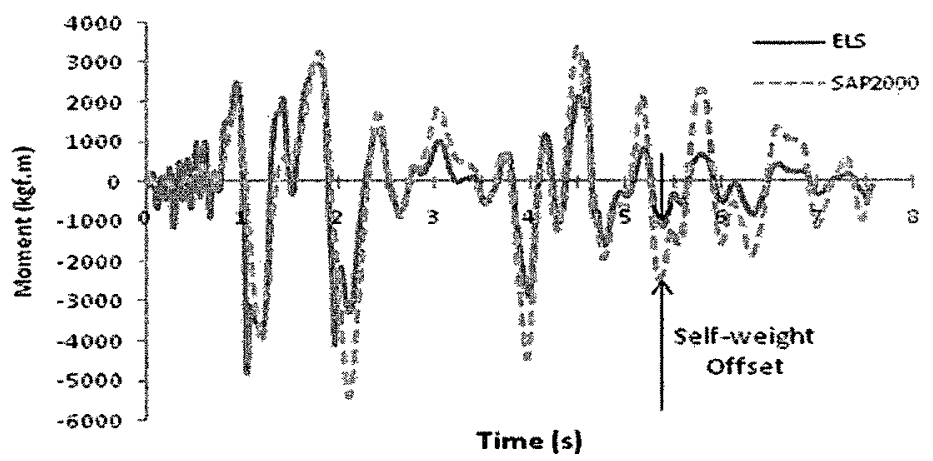

(b)

Figure 5.3. Nonlinear Analyses Verification Results: (a) Displacement, (b) Moment, (c) Shear Force, (d) Axial Force 


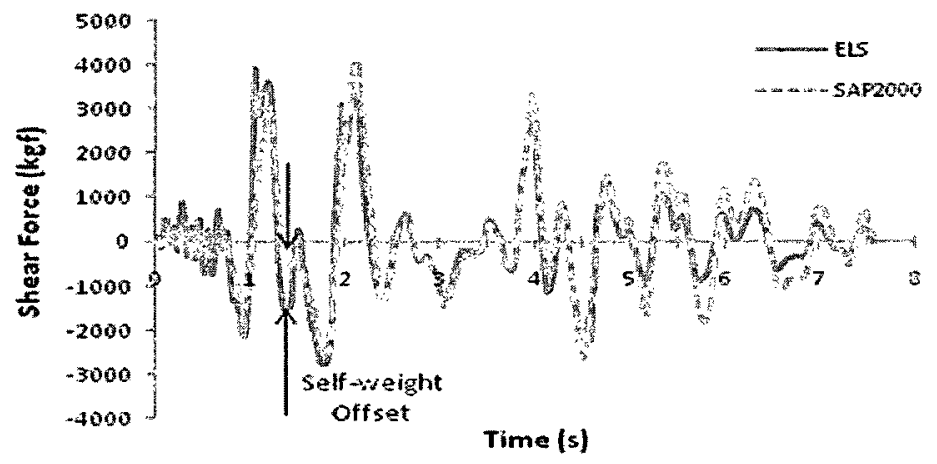

(c)

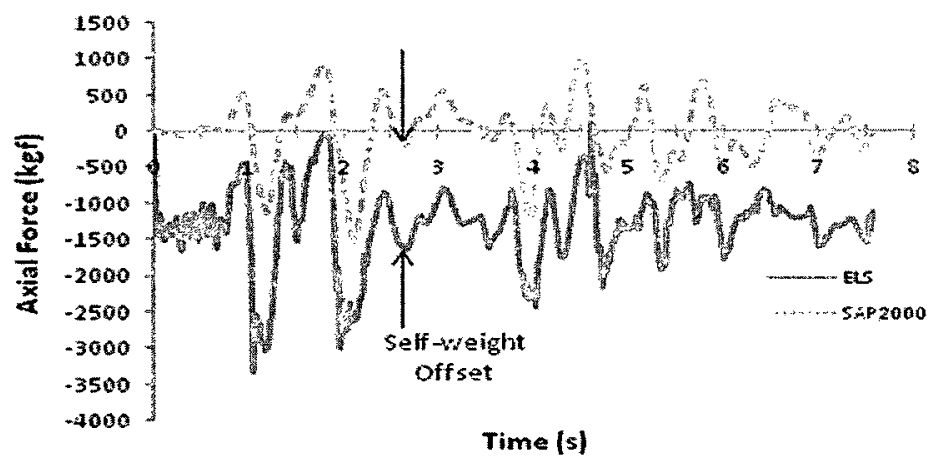

(d)

Figure 5.3. (cont' $d$ ) Nonlinear Analyses Verification Results: (a) Displacement, (b) Moment, (c) Shear Force, (d) Axial Force 


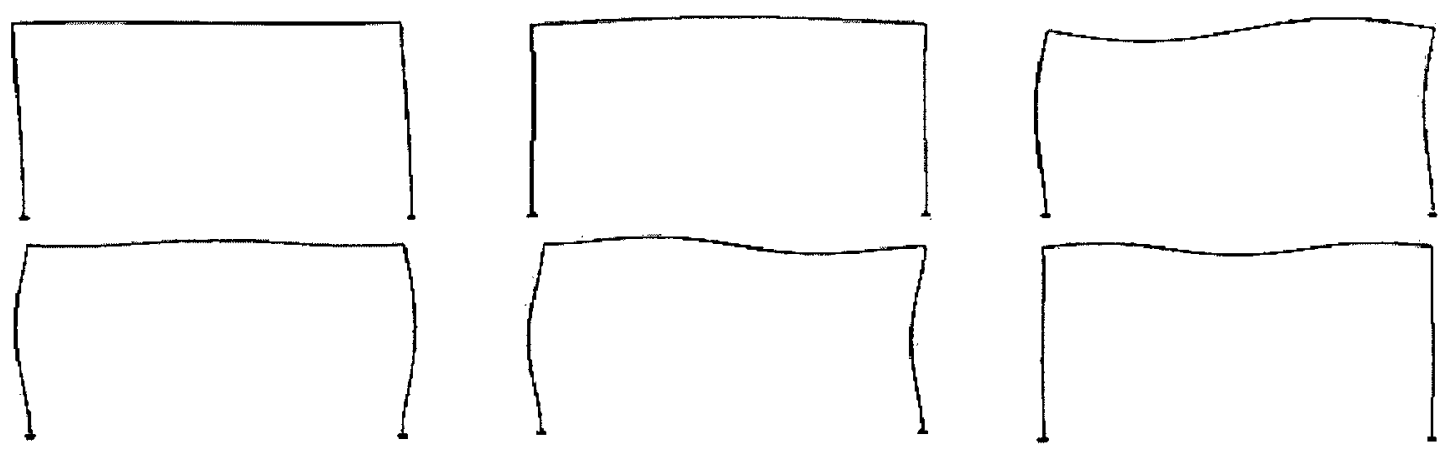

Figure 5.4. First Six Modal Shapes of the Verification Model 


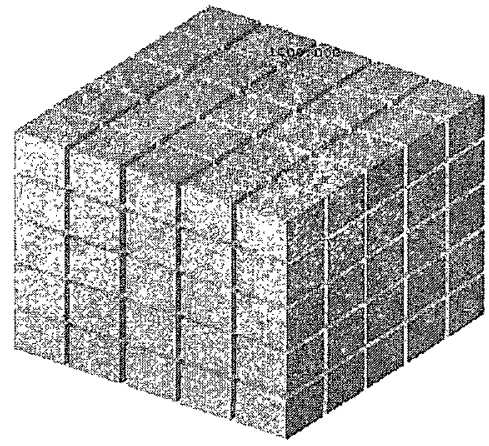

(a)

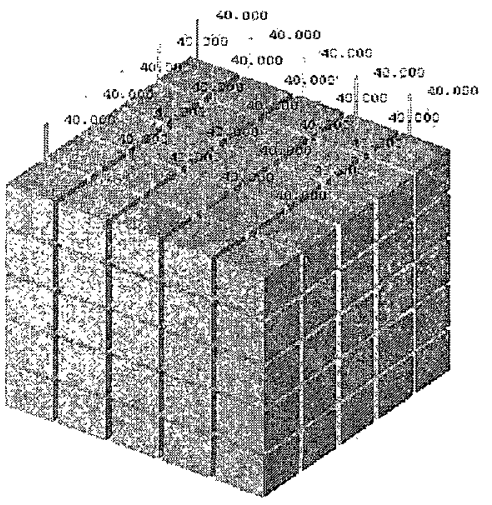

(b)

Figure 5.5. Location of the (a) Point and (b) Distributed Loads 


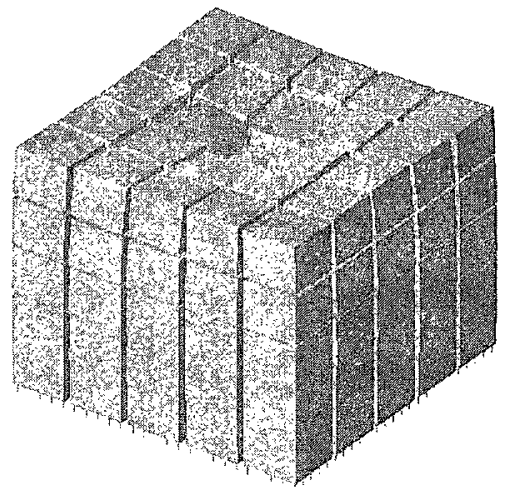

(a)

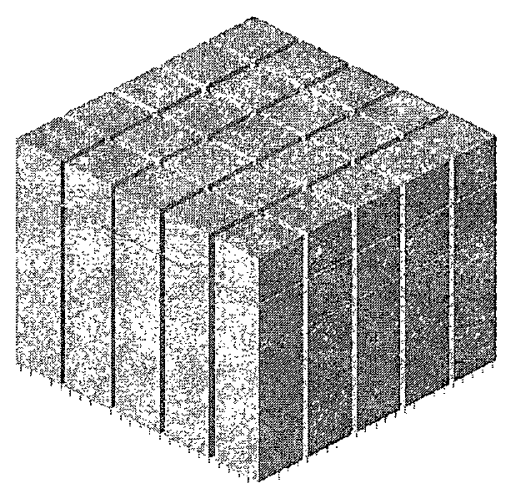

(b)

Figure 5.6. Deformations of Elements Caused by the (a) Point and (b) Distributed Loads 


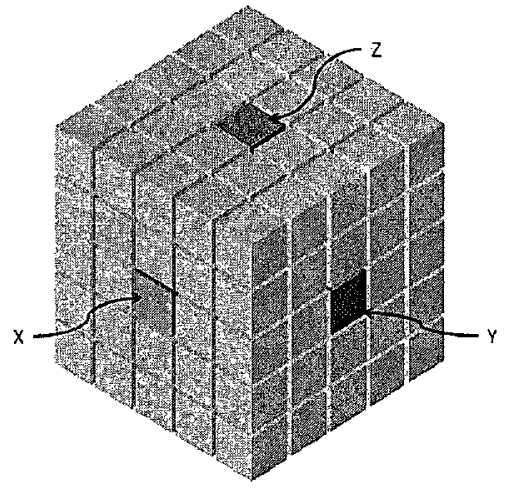

(a)

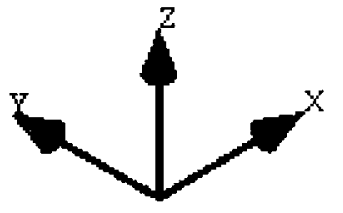

(b)

Figure 5.7. (a) Locations and (b) the Positive Global Axes of the Measured Elements 
(a)

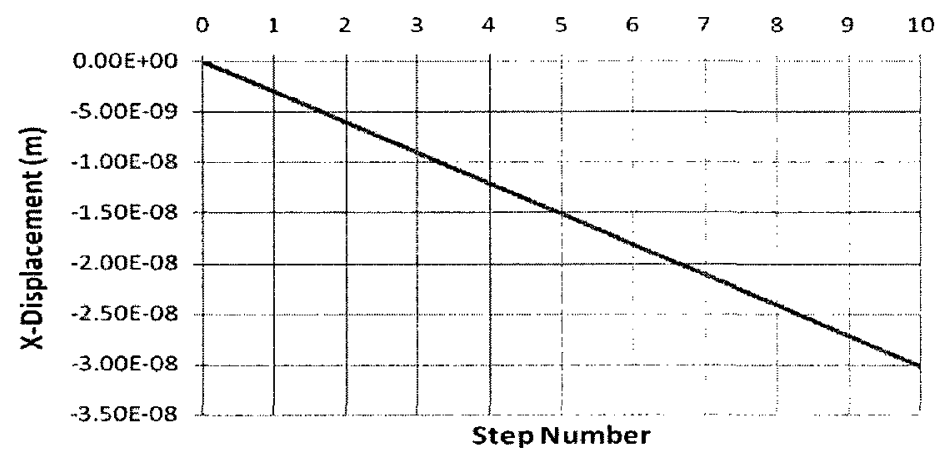

(b)

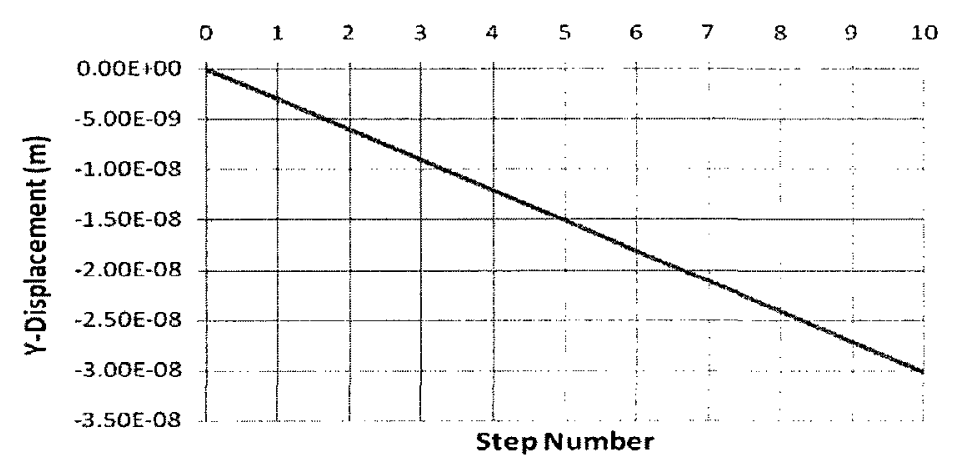

(c)

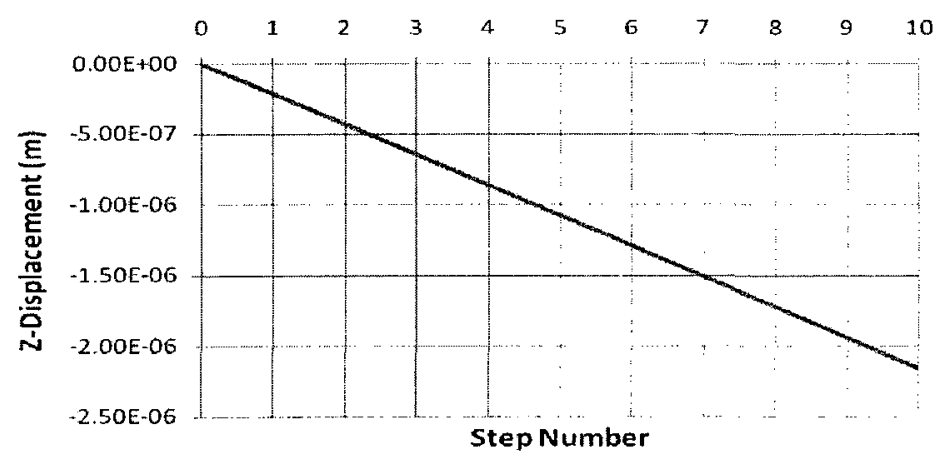

Figure 5.8. (a) X-, (b) Y-, and (c) Z-Displacements of the Elements Caused by the Point Load 
(a)

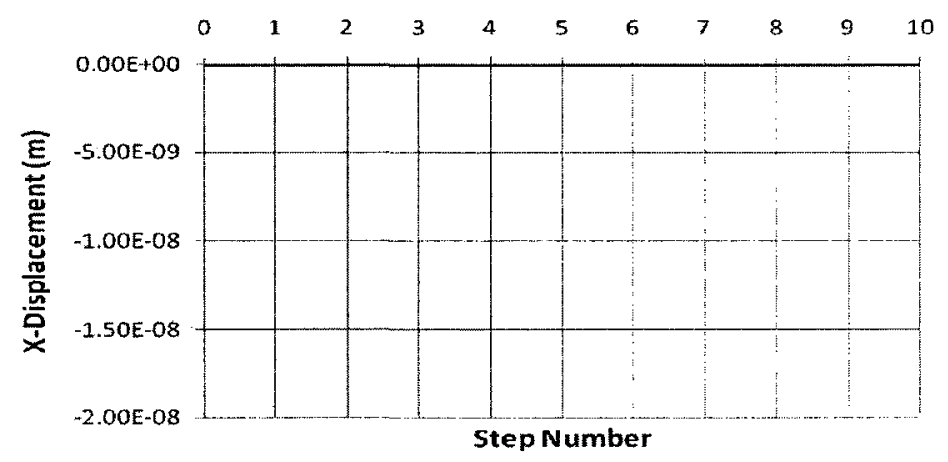

(b)

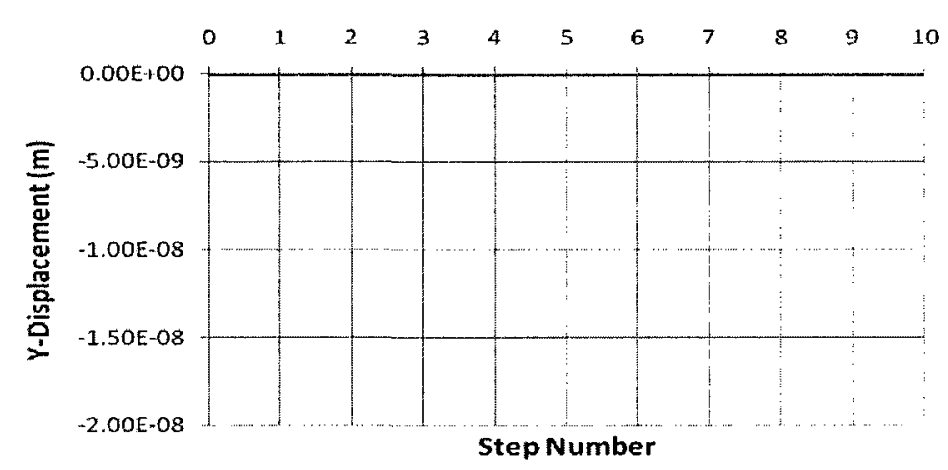

(c)

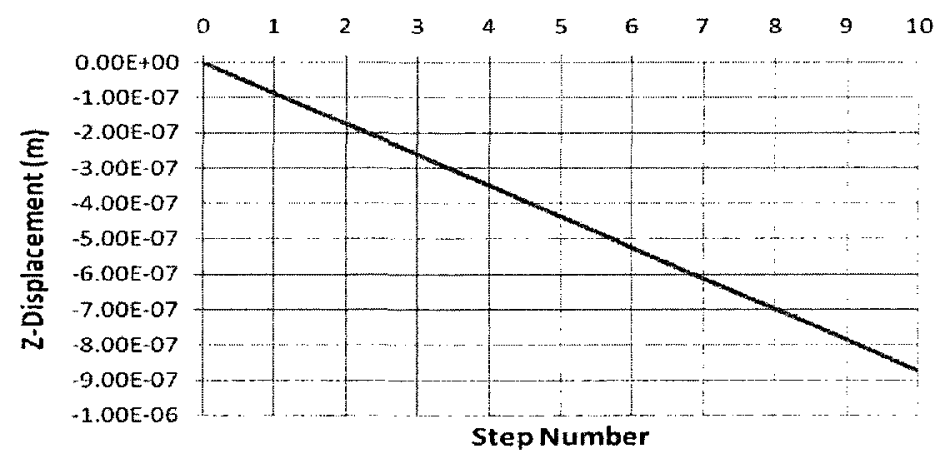

Figure 5.9. (a) X-, (b) Y-, and (c) Z-Displacements of the Elements Caused by the Distributed Load 
$\Xi$

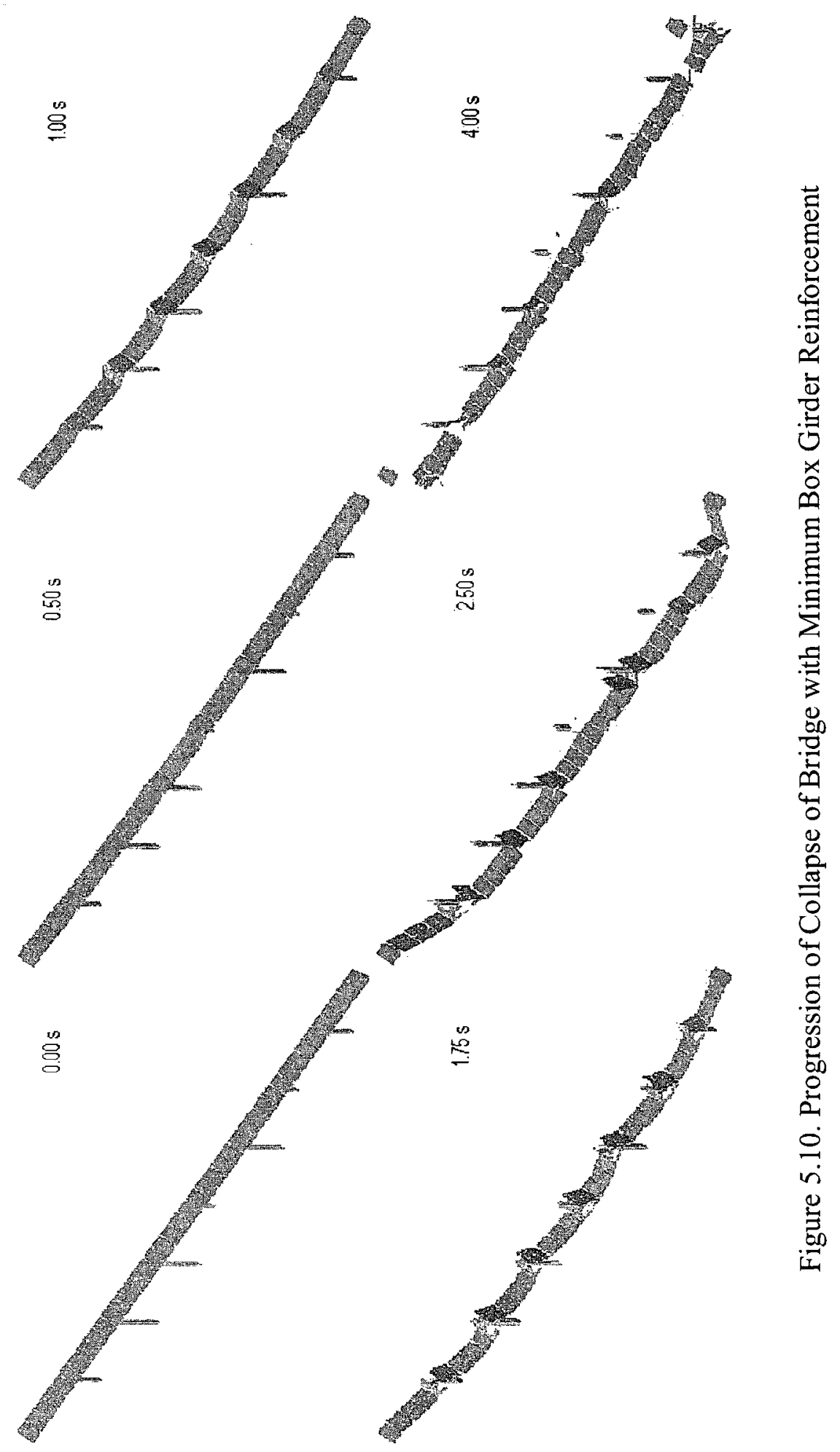




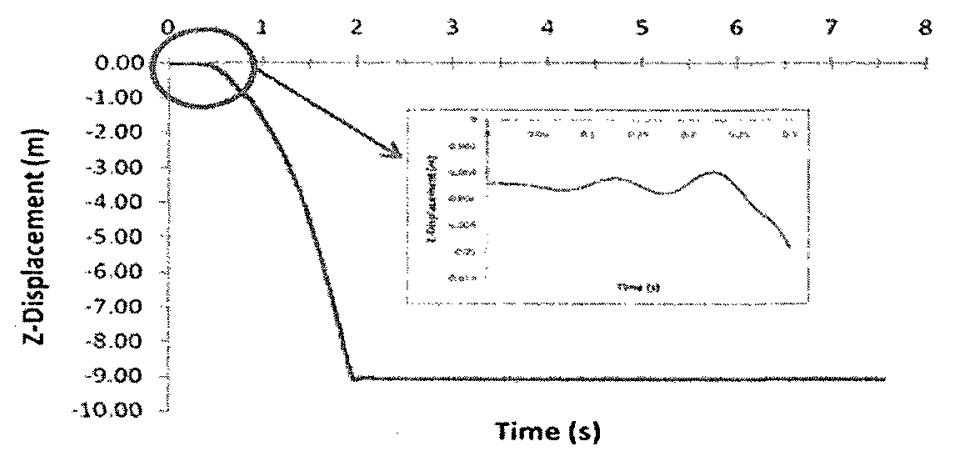

Figure 5.11. Displacement Time History along Z-Axis of the Middle Element of Deck E-F 


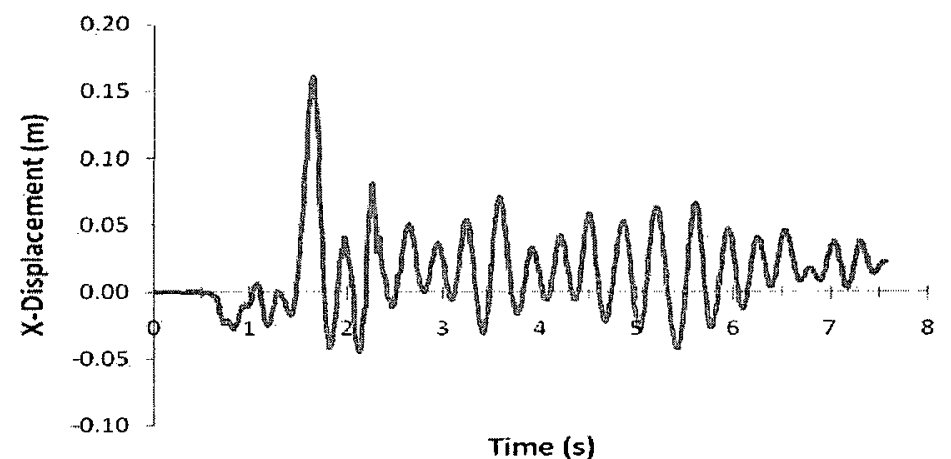

(a)

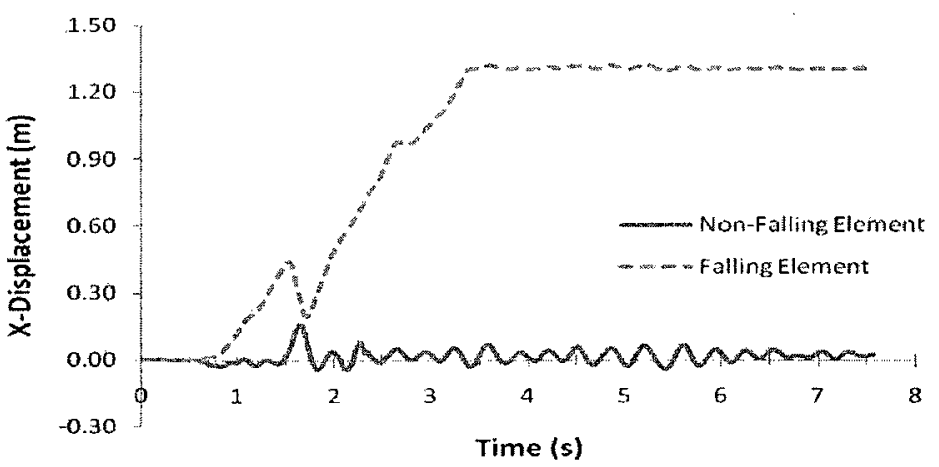

(b)

Figure 5.12. (a) Displacement Time History of the Non-Falling Element and (b) Comparison between the Non-Falling and Falling Elements along X-Axis at the Top of Pier F 


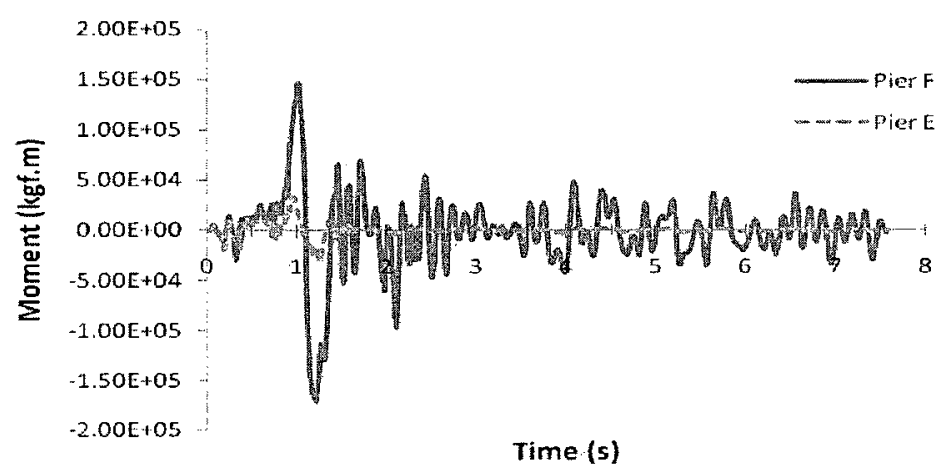

Figure 5.13. Overturning Moment Time History about $\mathrm{Y}$-Axis at the Base of Piers E and $\mathrm{F}$ 


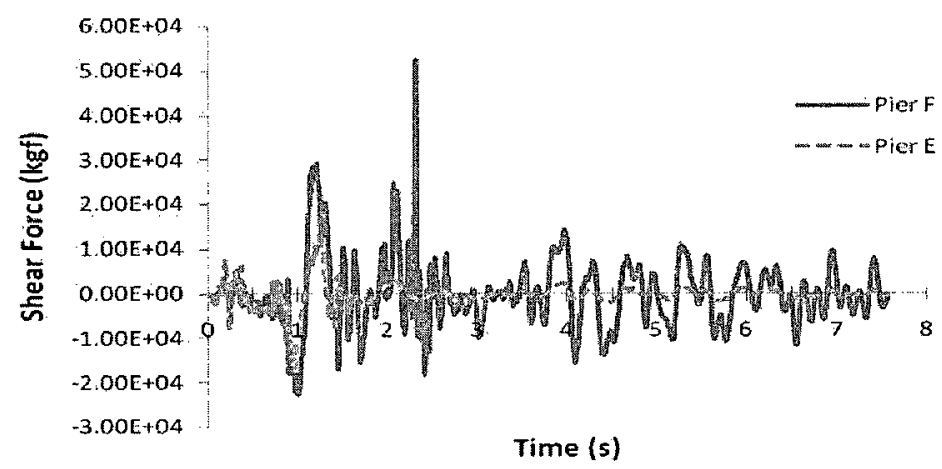

Figure 5.14. Shear Force Time History about X-Axis at the Base of Piers E and F 


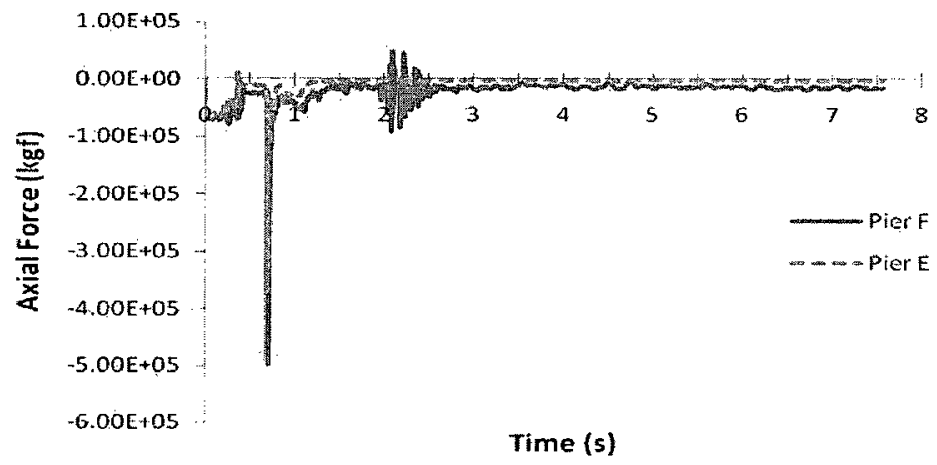

Figure 5.15. Axial Force Time History at the Base of Piers E and F 

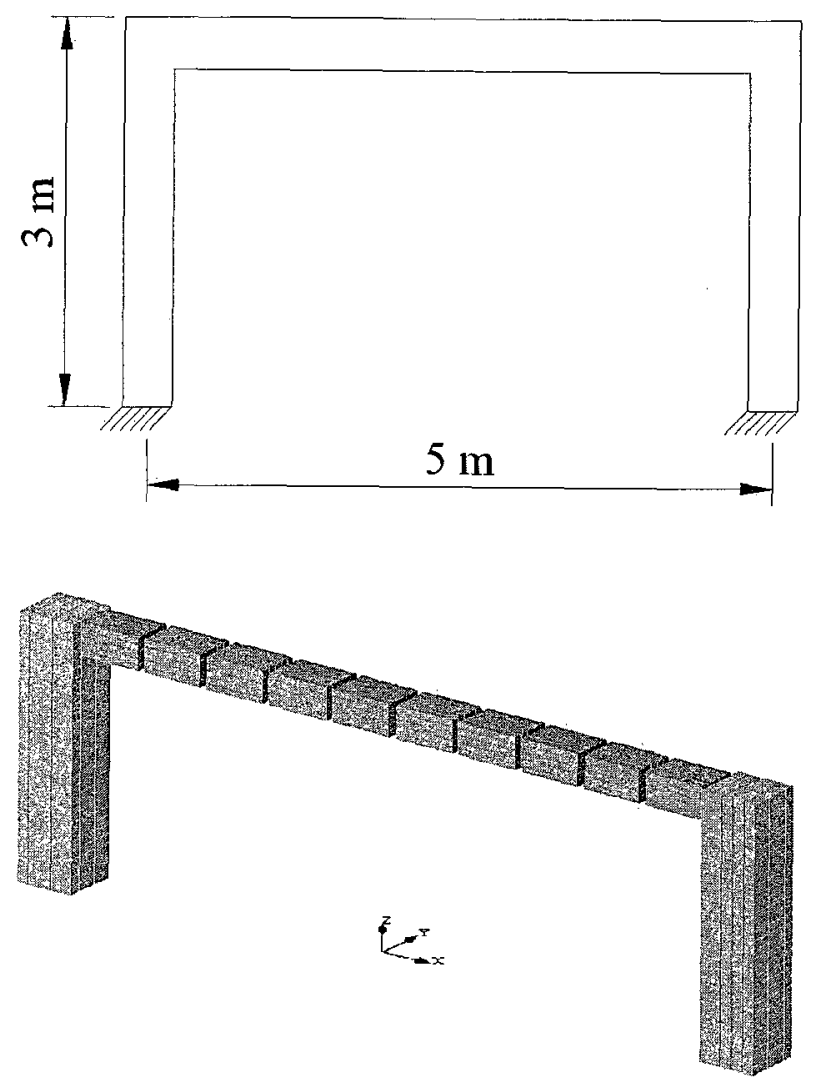

Figure 5.16. (a) Elevation and (b) 3D Views of the Viaduct Model 

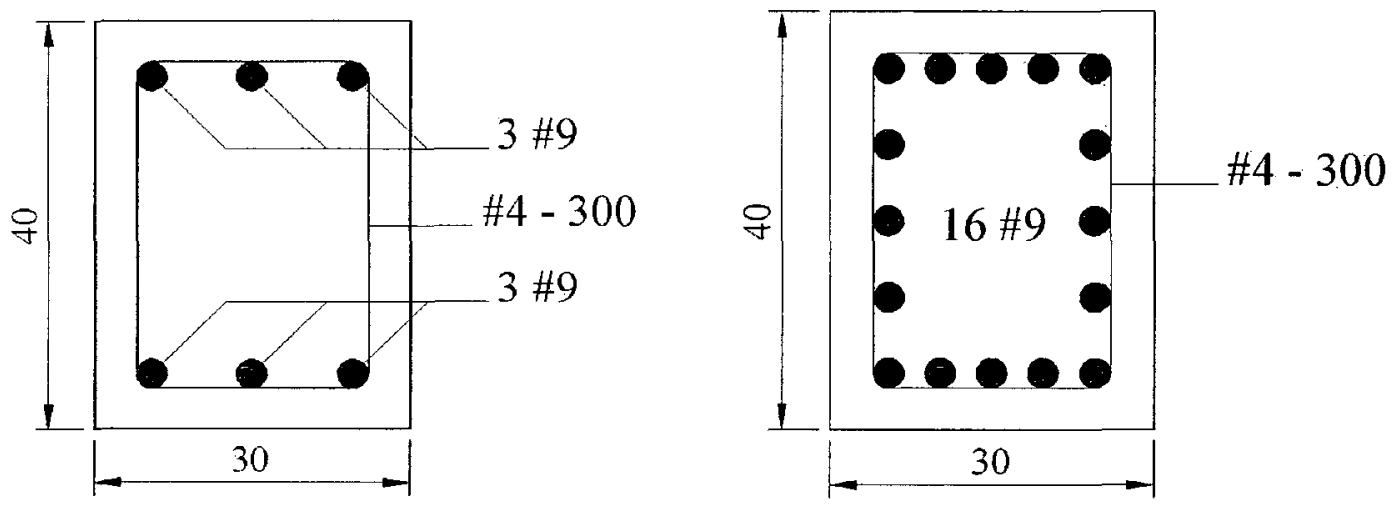

(units in $\mathrm{mm}$ )

(a)

(b)

Figure 5.17. Two Types of Viaduct's Beam: (a) B1 and (b) B2 


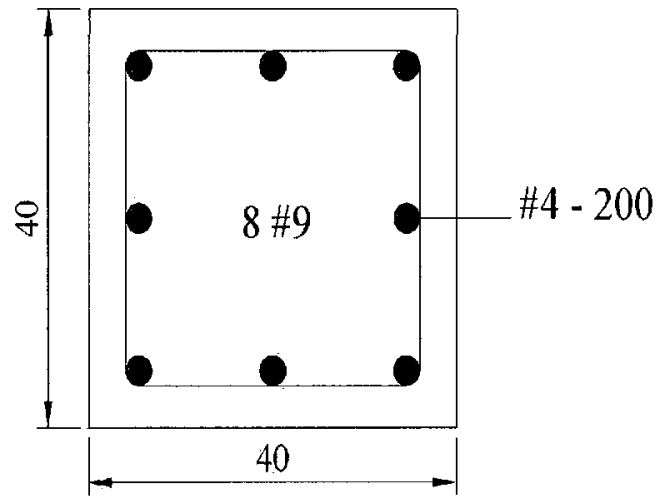

(units in mm)

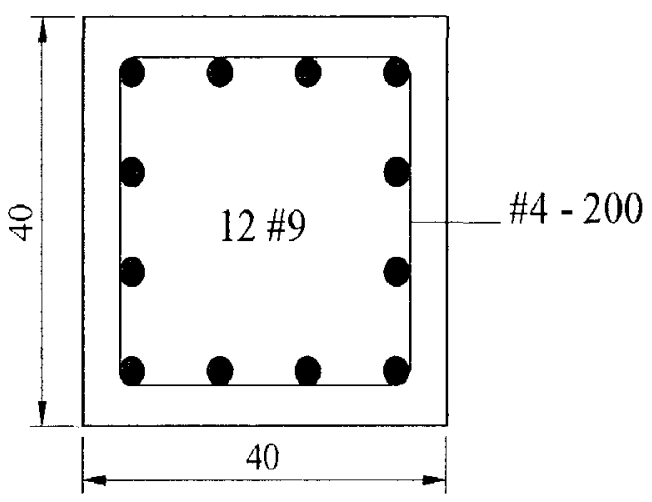

(a)

(b)

Figure 5.18. Two Types of Viaduct's Column: (a) $\mathrm{C} 1$ and (b) $\mathrm{C} 2$ 


$$
\begin{aligned}
& \text { EE } \\
& \text { EE } \\
& \text { CEL }
\end{aligned}
$$


$\Phi$
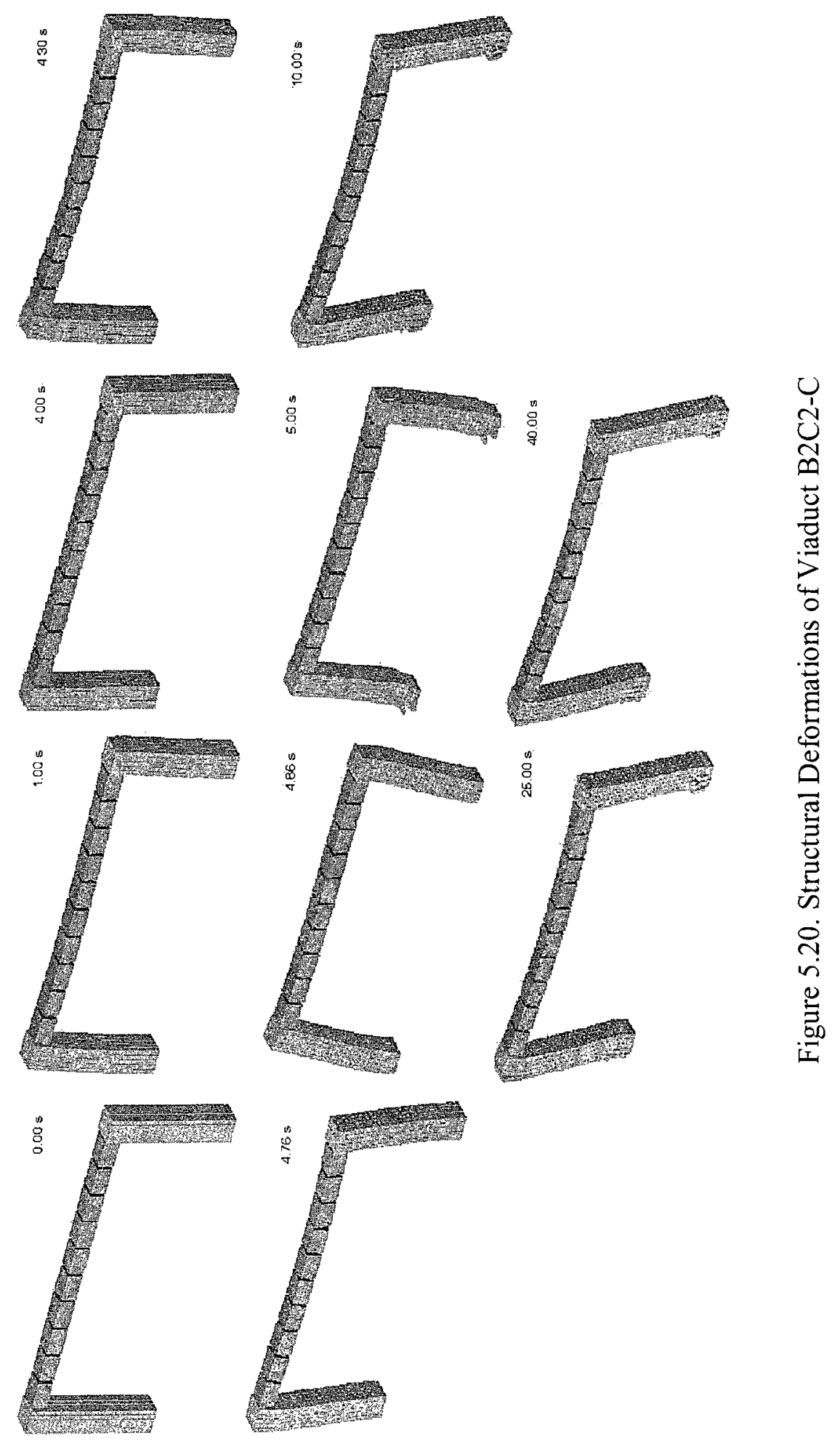


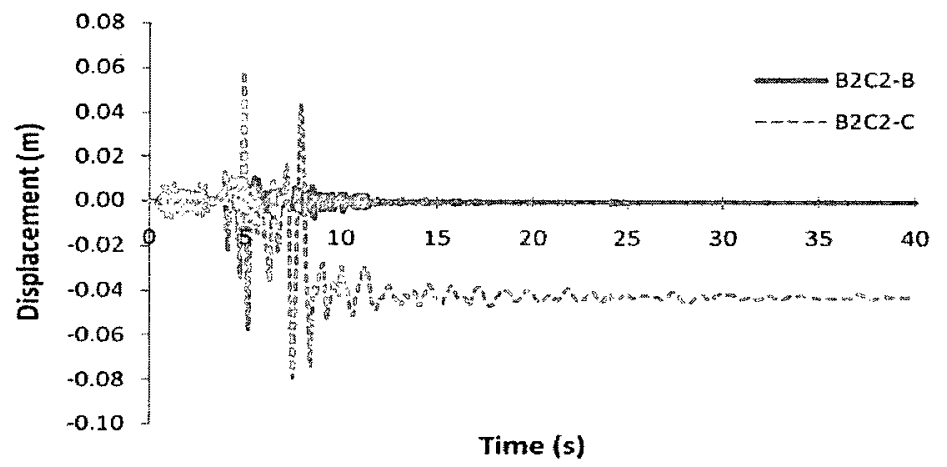

(a)

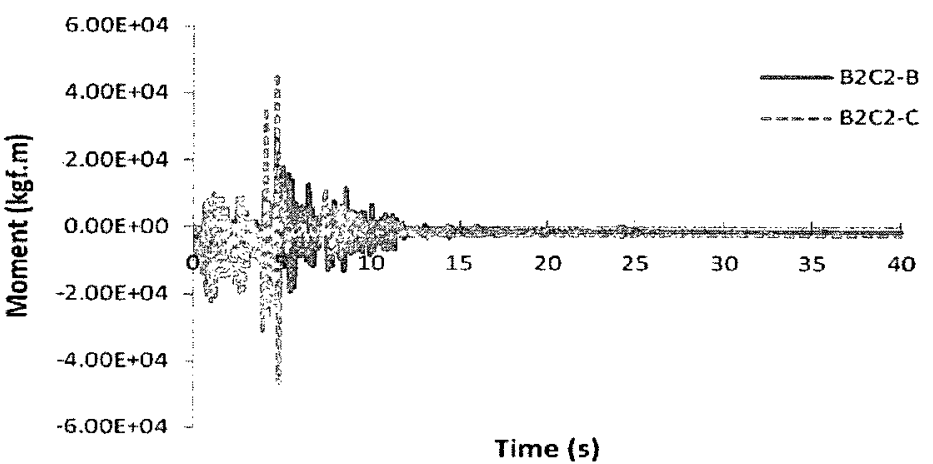

(b)

Figure 5.21. (a) Top Displacement, (b) Overturning Moment, (c) Shear Force, and (d) Axial Force Time Histories for Viaducts B2C2-B and B2C2-C 


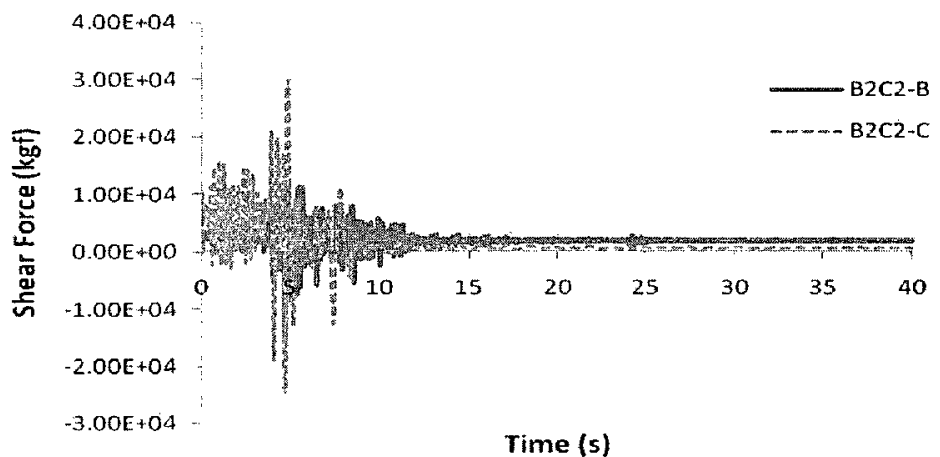

(c)

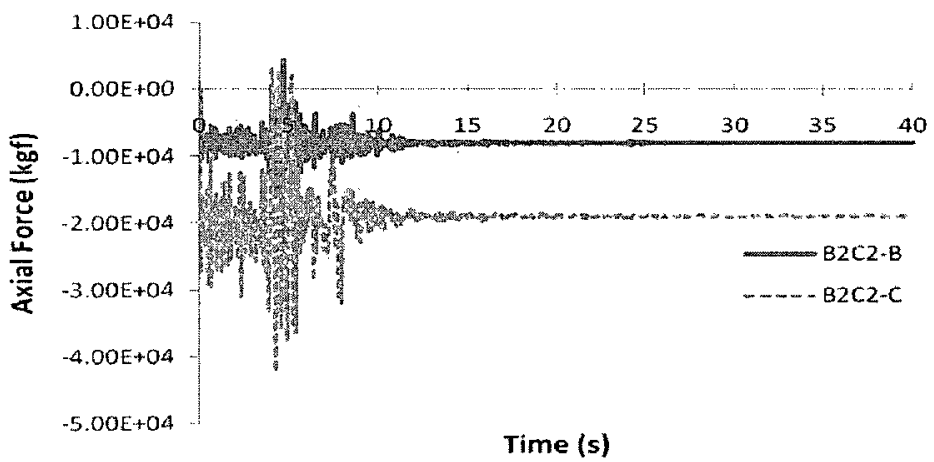

(d)

Figure 5.21. (cont'd) (a) Top Displacement, (b) Overturning Moment, (c) Shear Force, and (d) Axial Force Time Histories for Viaducts B2C2-B and B2C2-C 
$\stackrel{+}{\infty}$
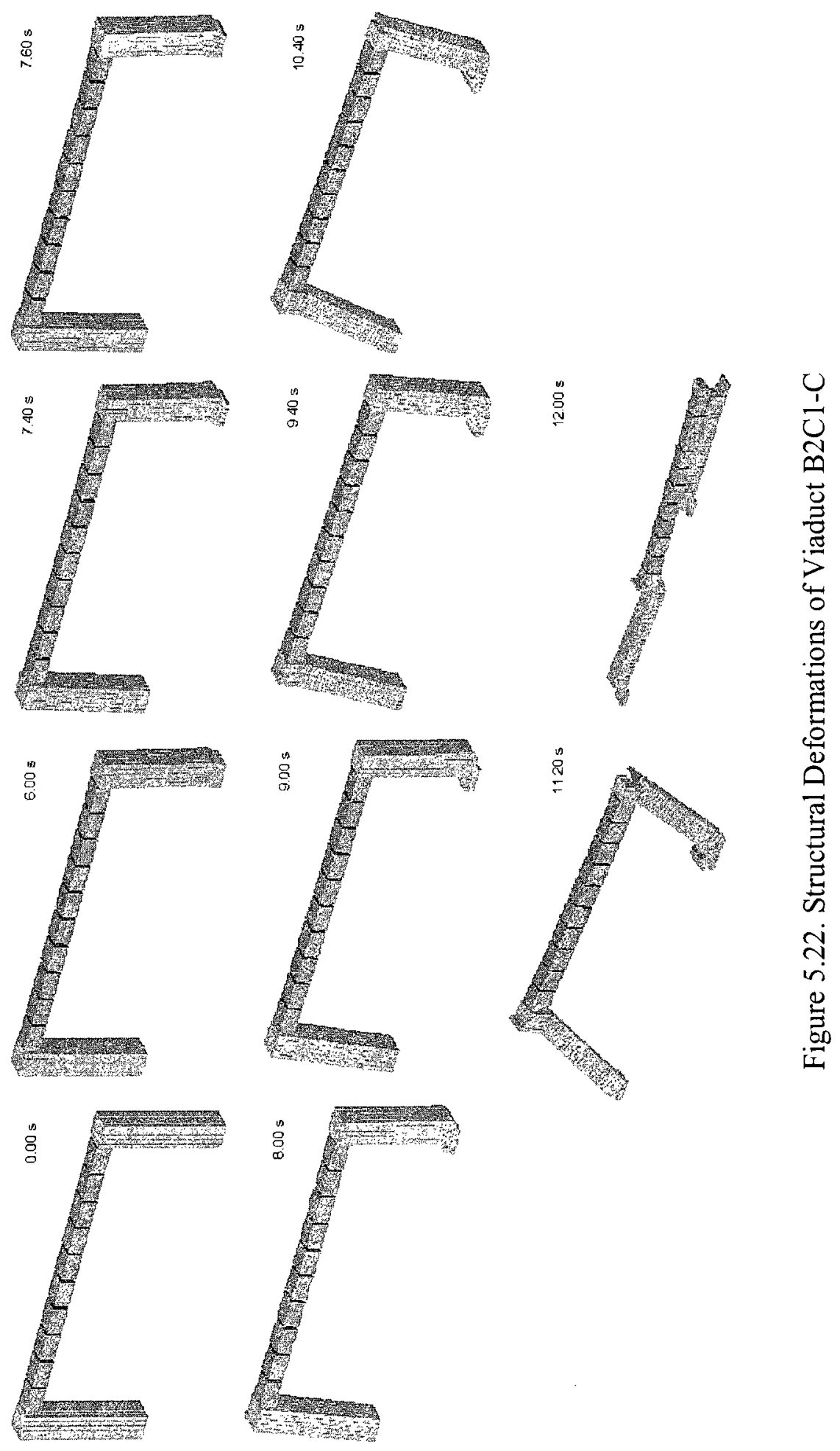
$\infty$
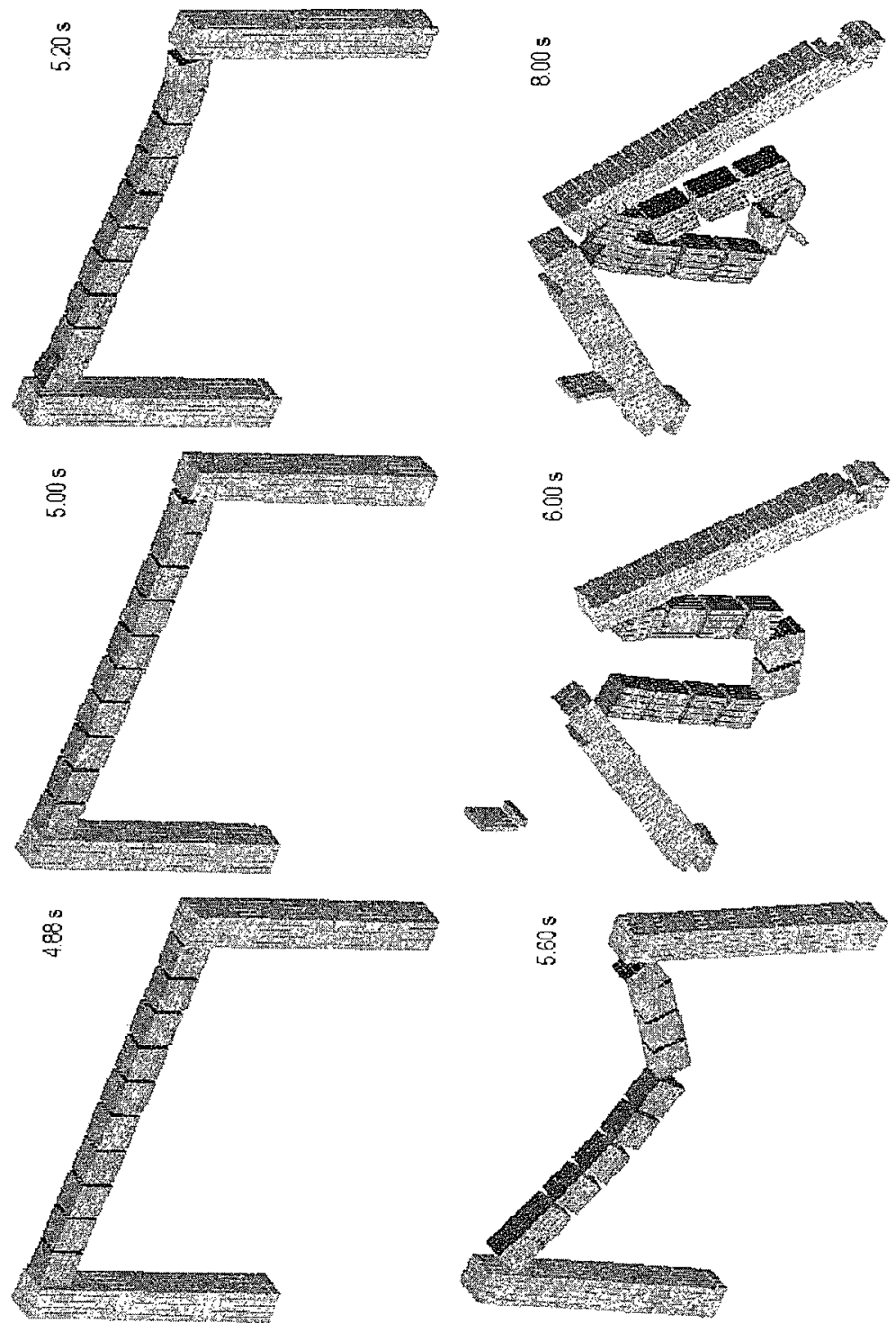

Uे
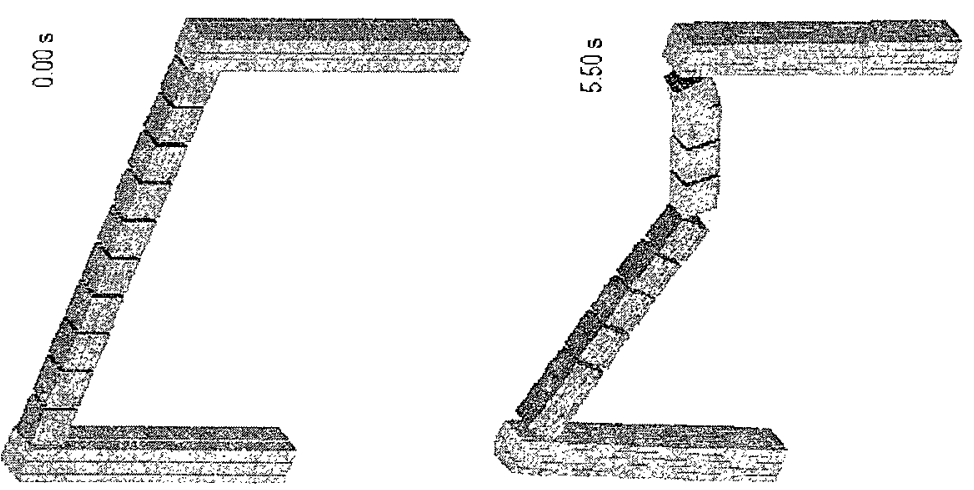
(a)
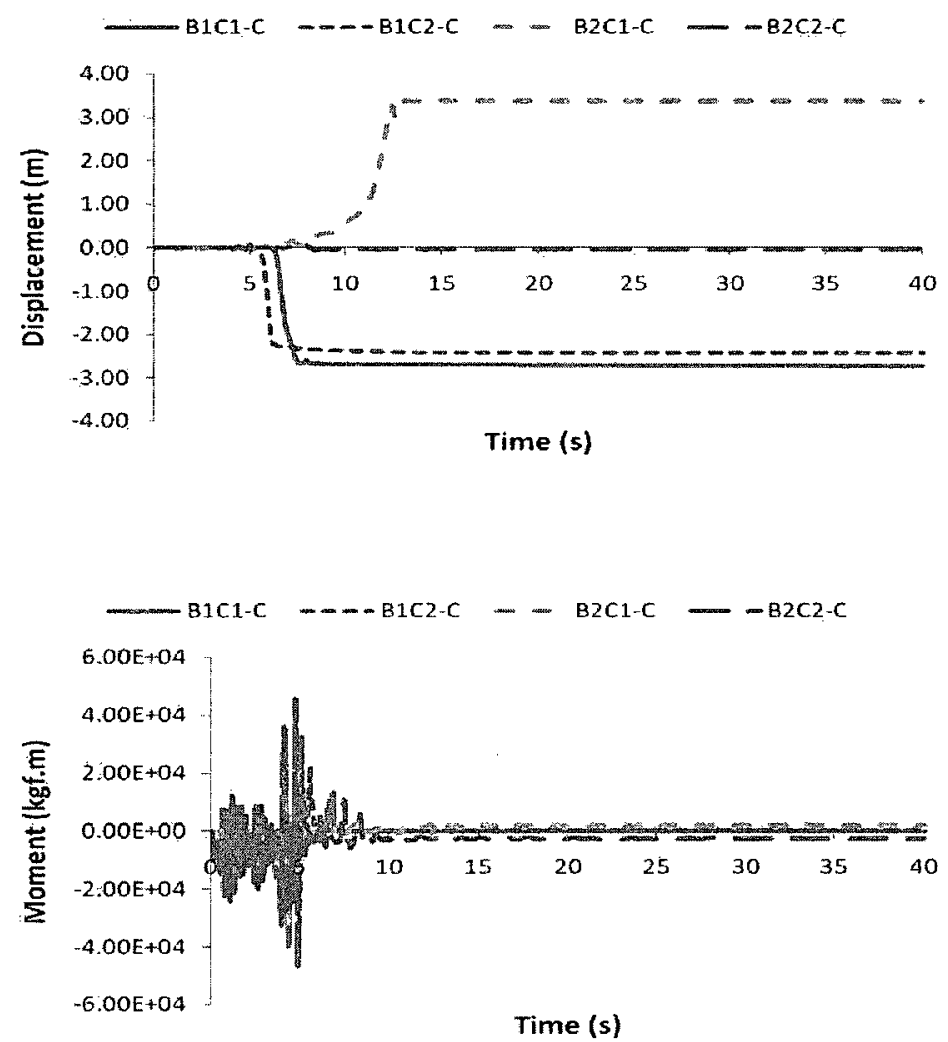

(b)

Figure 5.24. (a) Top Displacement, (b) Overturning Moment, (c) Shear Force, and (d) Axial Force Time Histories for Viaducts B1C1-C, B1C2-C, B2C1-C, and B2C2-C 


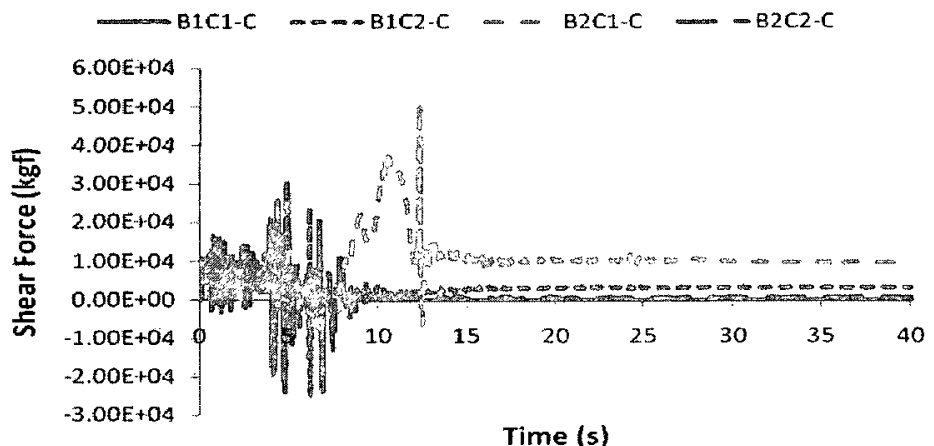

(c)

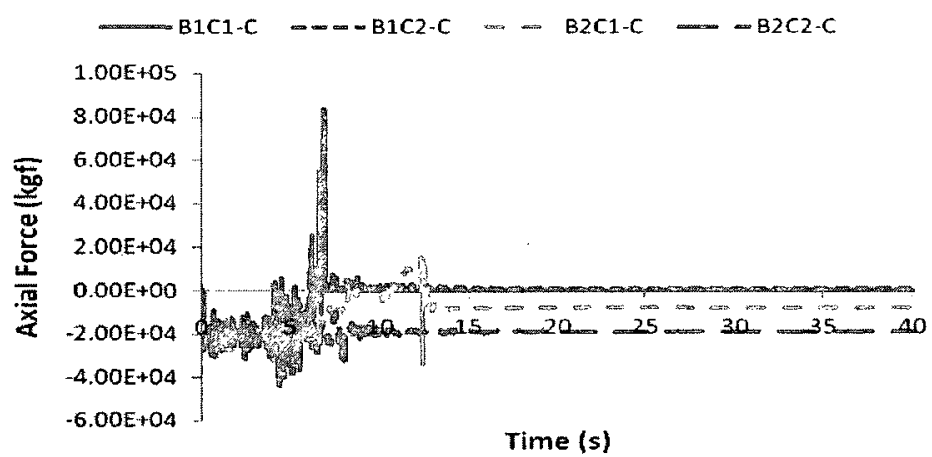

(d)

Figure 5.24. (cont'd) (a) Top Displacement, (b) Overturning Moment, (c) Shear Force, and (d) Axial Force Time Histories for Viaducts B1C1-C, B1C2-C, B2C1-C, and $\mathrm{B} 2 \mathrm{C} 2-\mathrm{C}$ 


$$
\text { 萑 }
$$


$\stackrel{\infty}{ }$
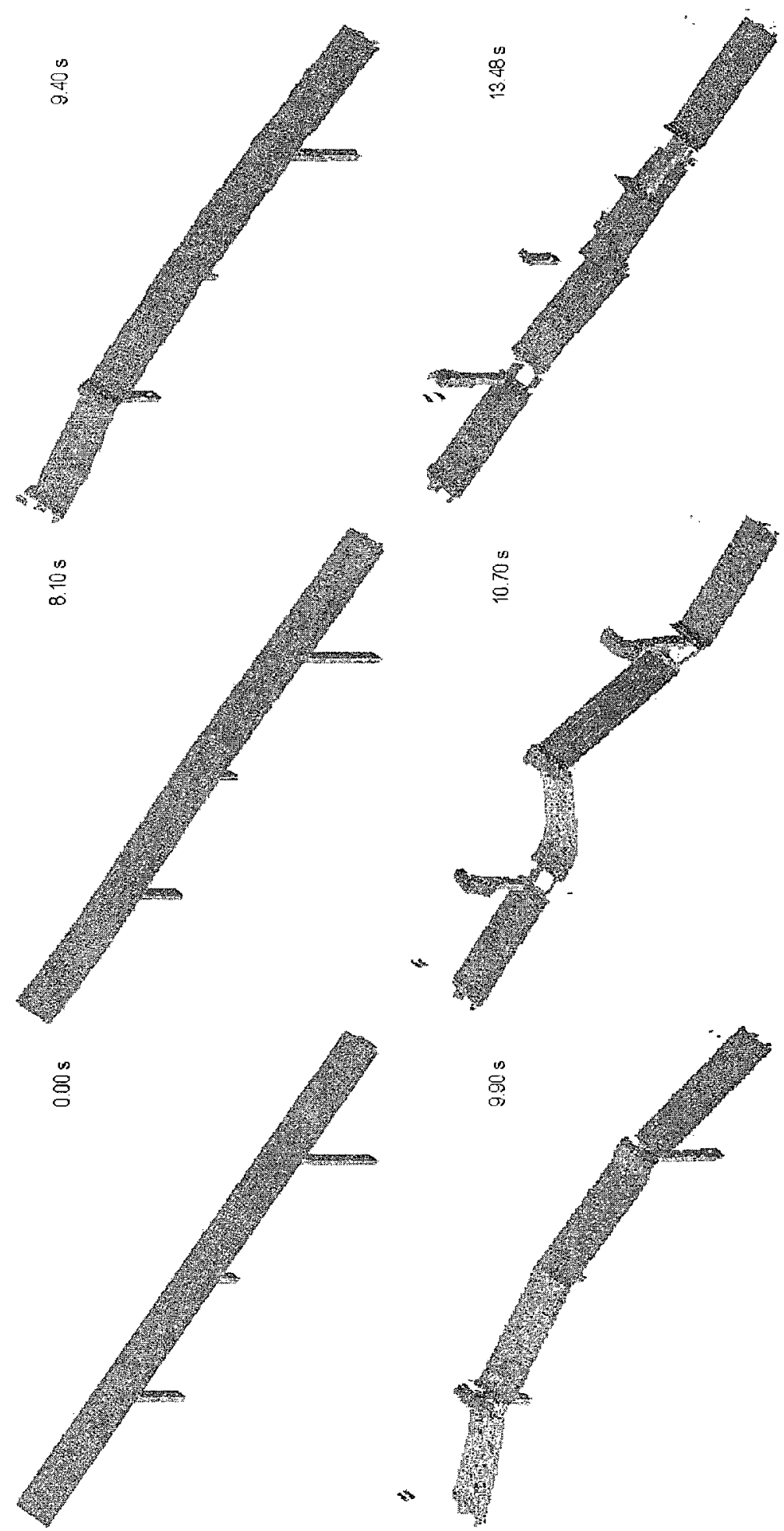

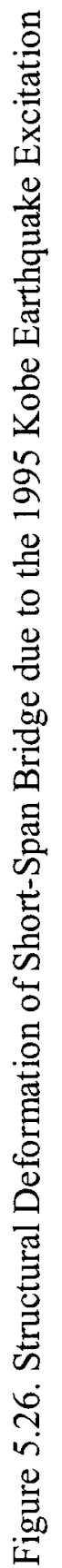


\&
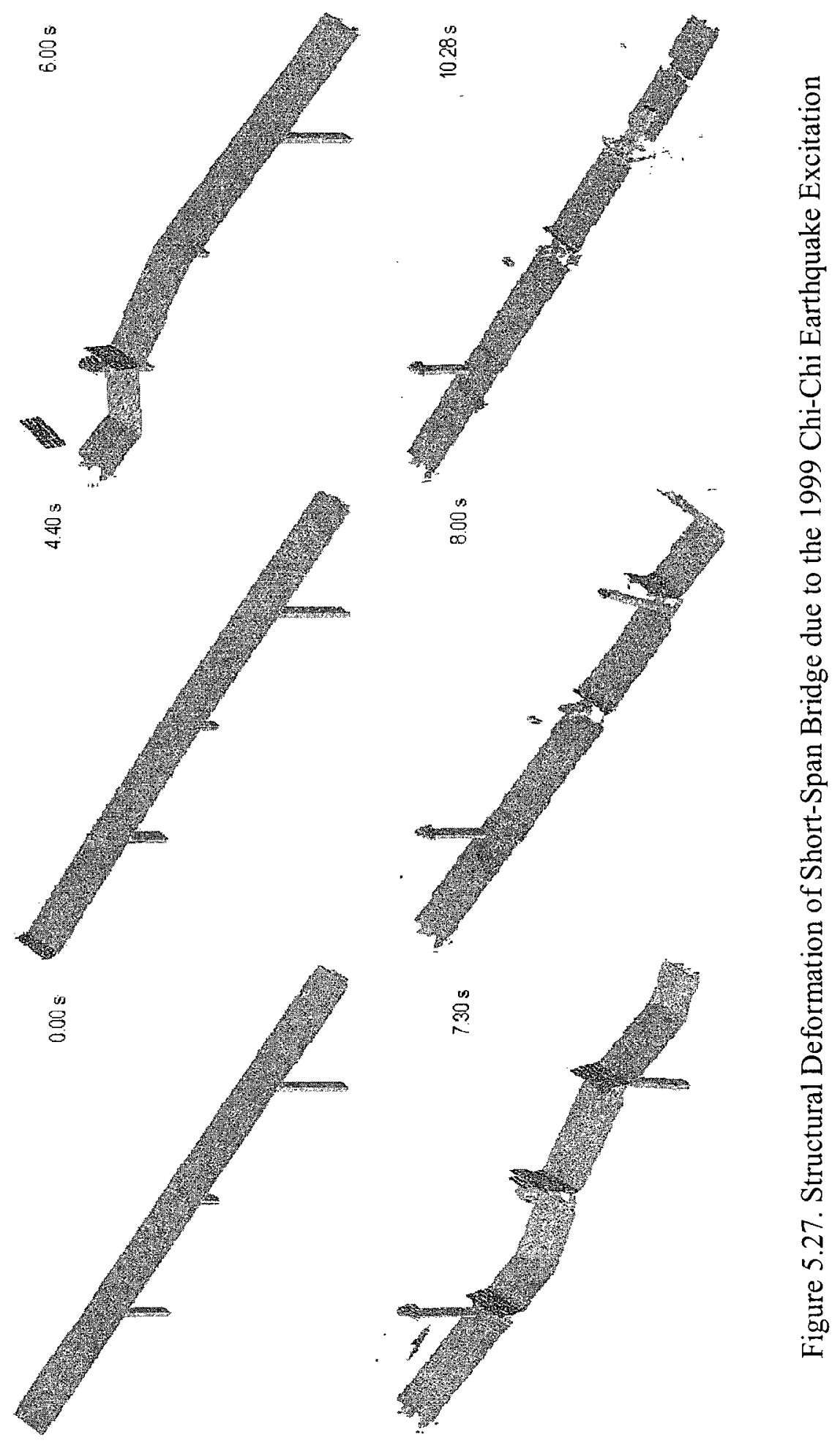


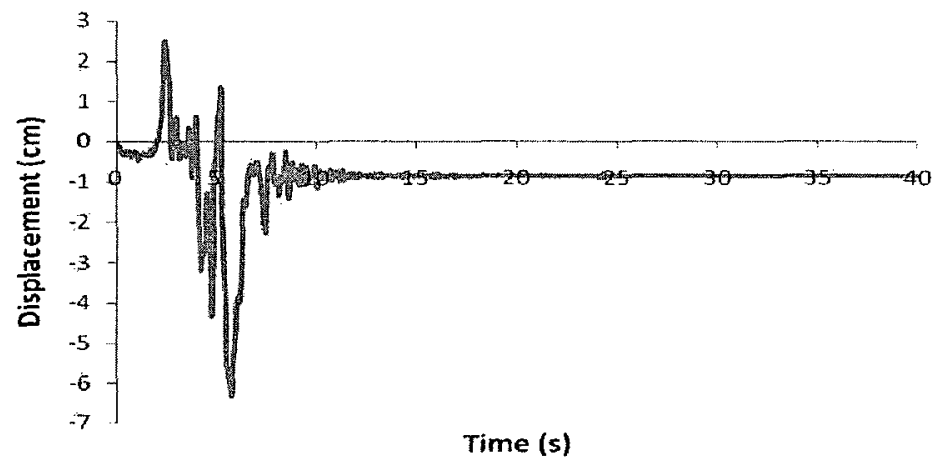

(a)

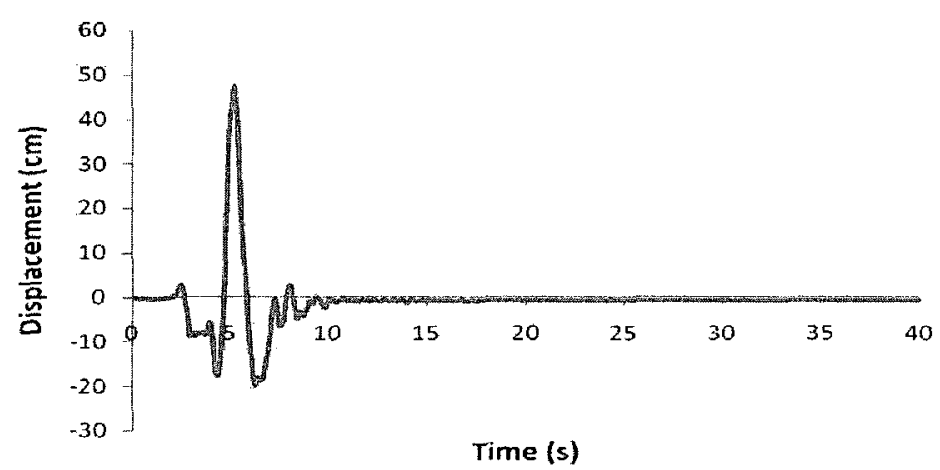

(b)

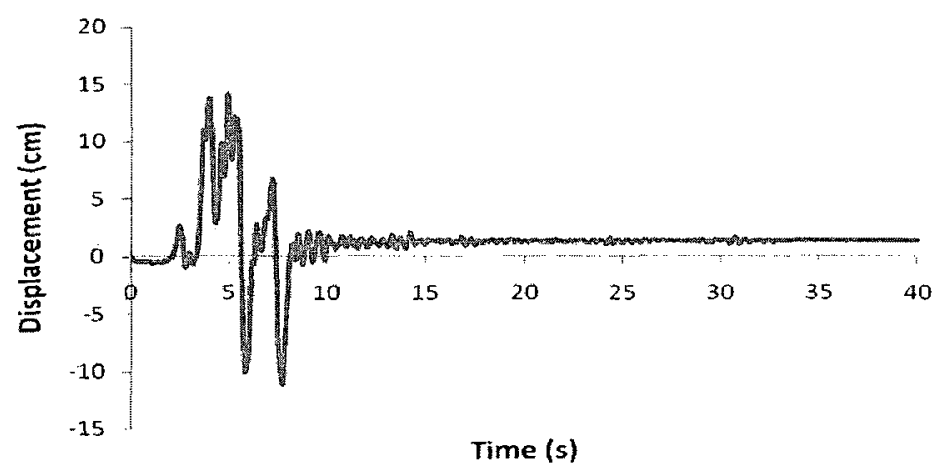

(c)

Figure 5.28. (a) Short, (b) Medium, and (c) Tall Piers' Top Displacement Time Histories along the X-Axis due to the 1994 Northridge Earthquake Excitation 


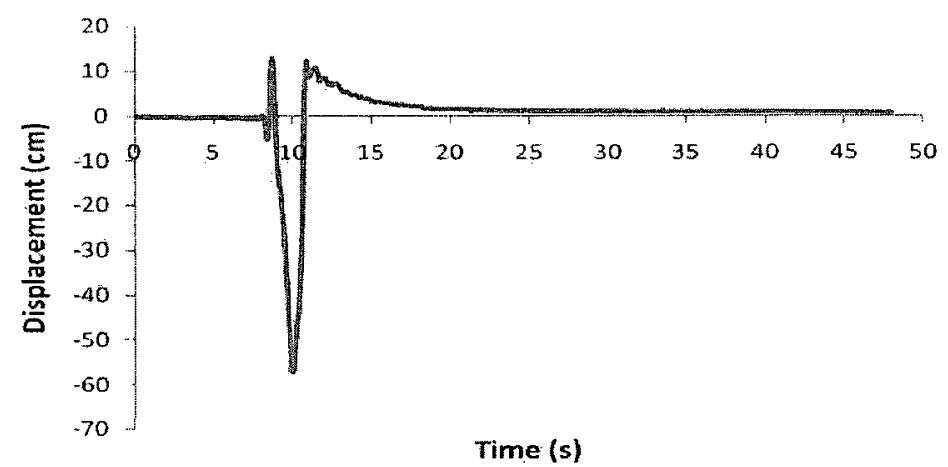

(a)

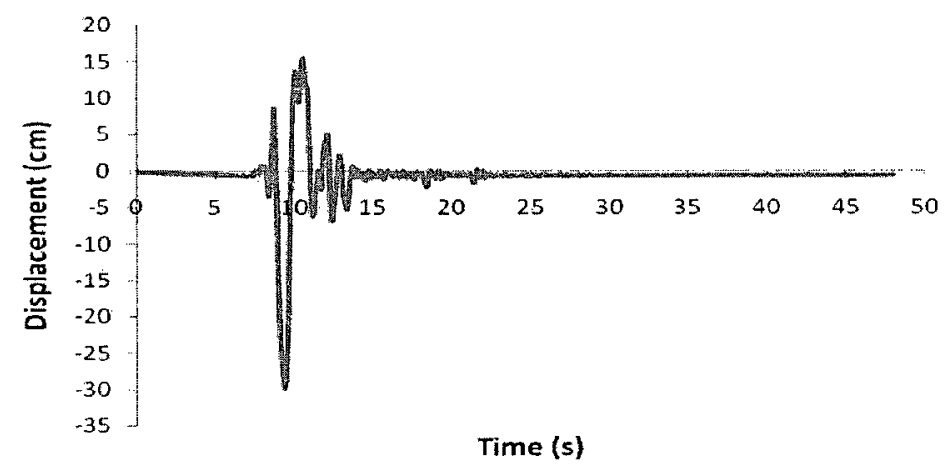

(b)

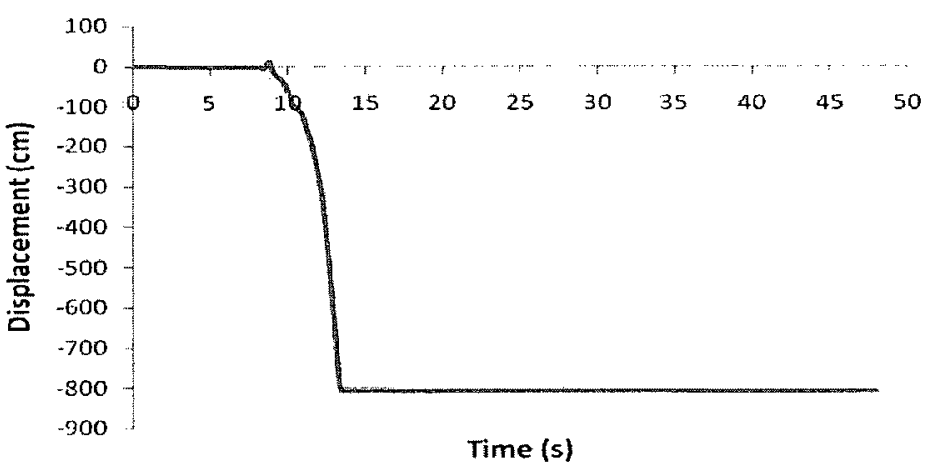

(c)

Figure 5.29. (a) Short, (b) Medium, and (c) Tall Piers' Top Displacement Time Histories along the X-Axis due to the 1995 Kobe Earthquake Excitation 
(a)
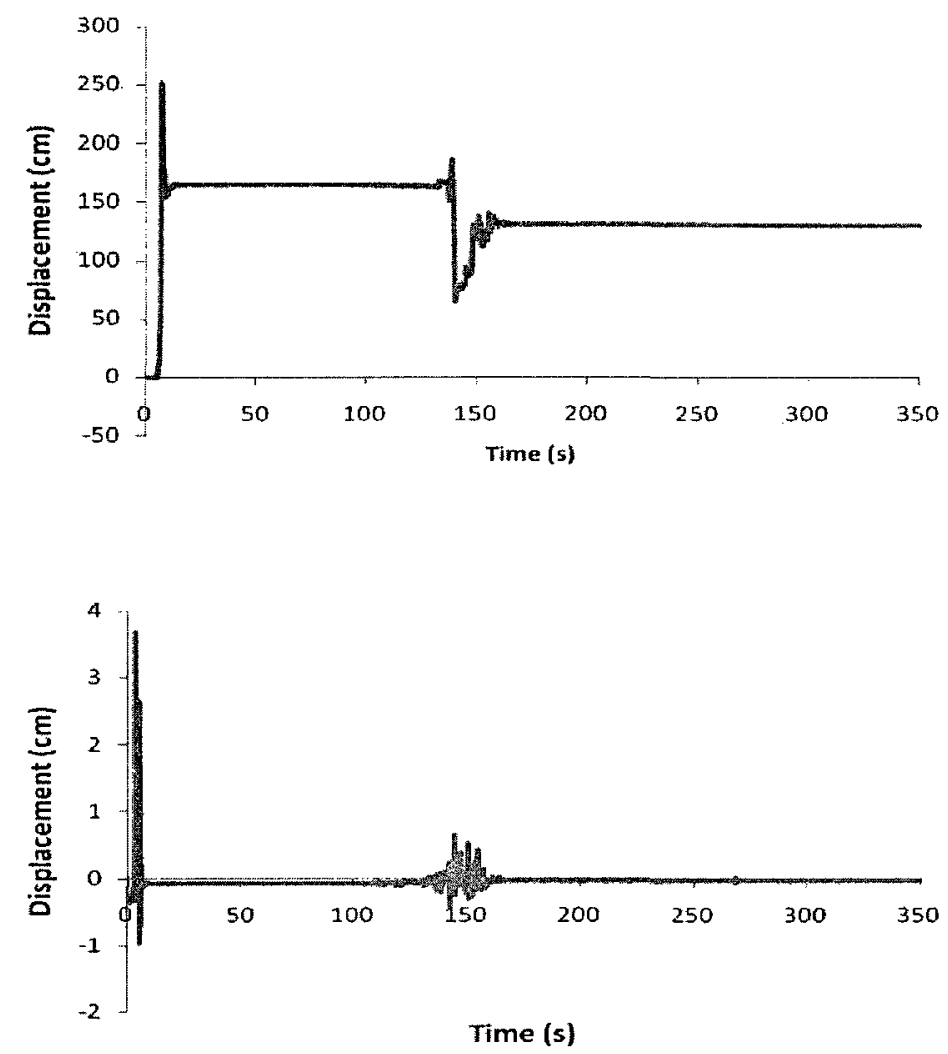

(b)

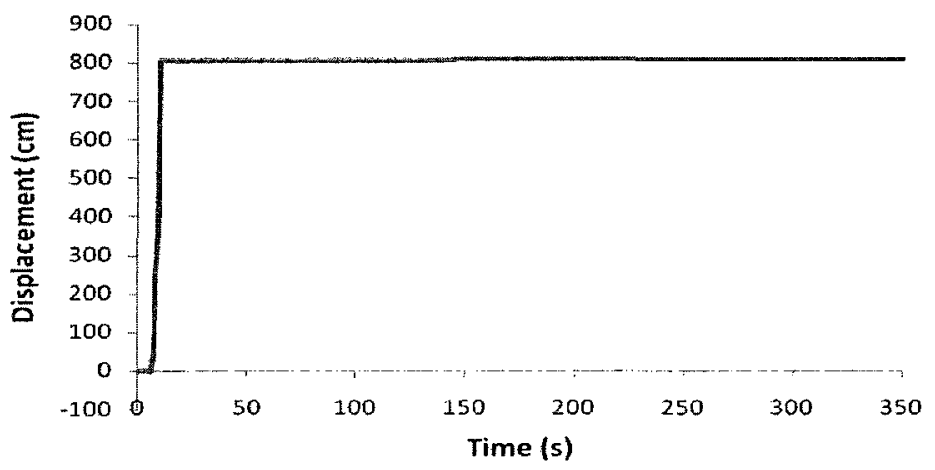

(c)

Figure 5.30. (a) Short, (b) Medium, and (c) Tall Piers' Top Displacement Time Histories along the X-Axis due to the 1999 Chi-Chi Earthquake Excitation 


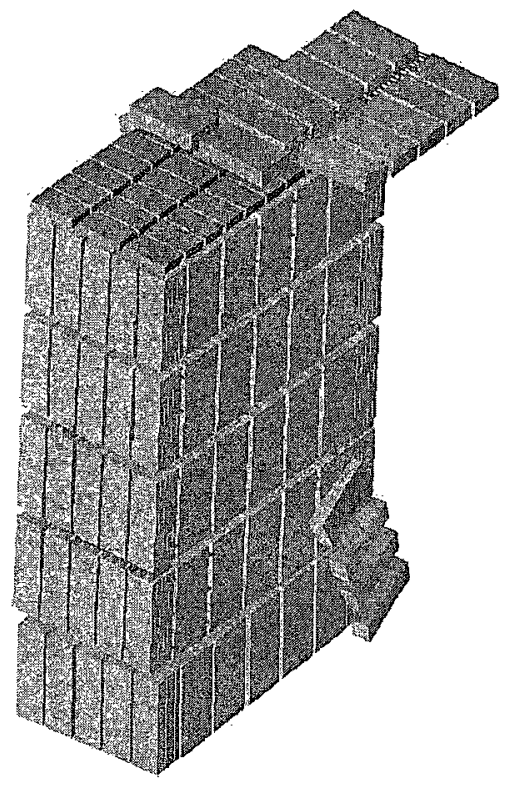

Figure 5.31. Dislocation and Cracks of the Short Pier due to the 1995 Kobe Earthquake Excitation 


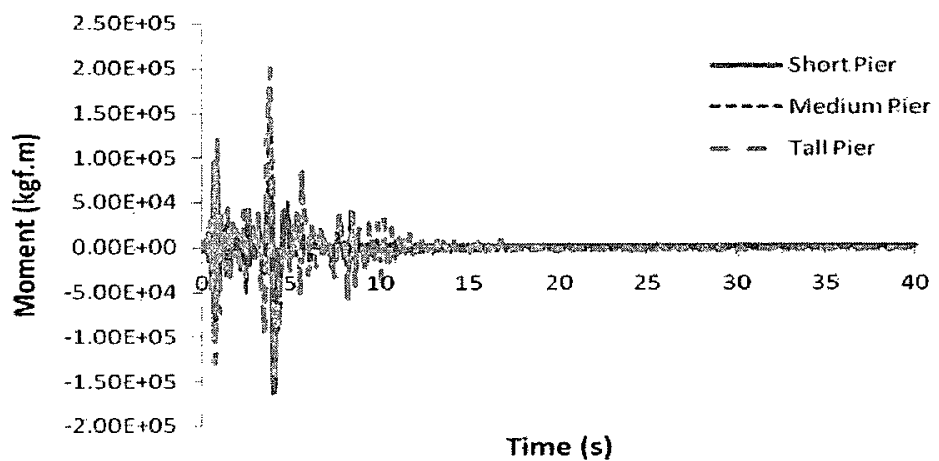

(a)

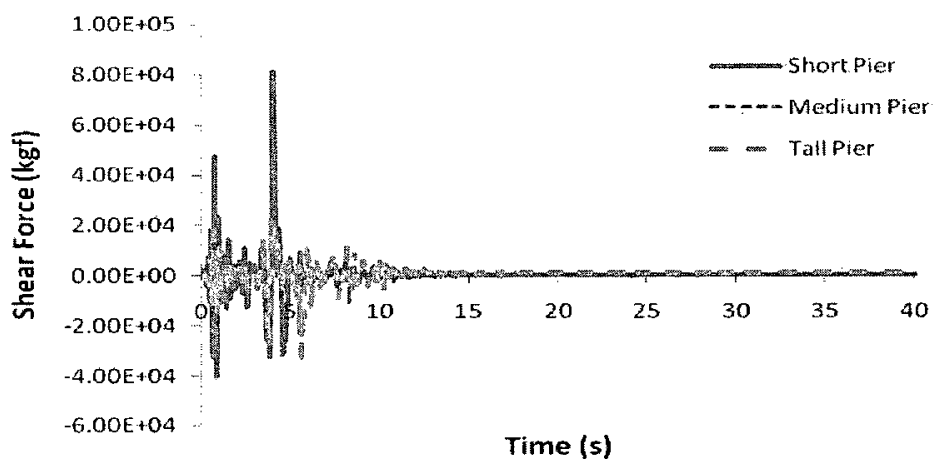

(b)

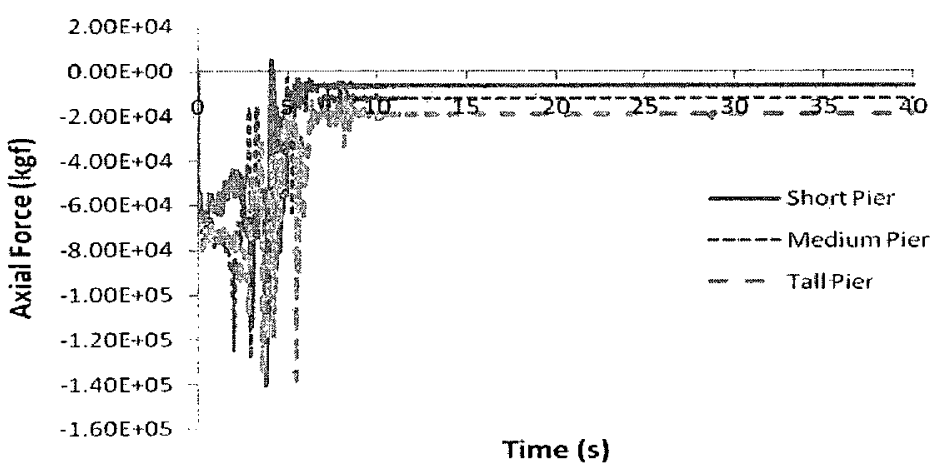

(c)

Figure 5.32. (a) Moment, (b) Shear Force, and (c) Axial Force Time Histories at the Base of the Piers due to the 1994 Northridge Earthquake Excitation 
(a)
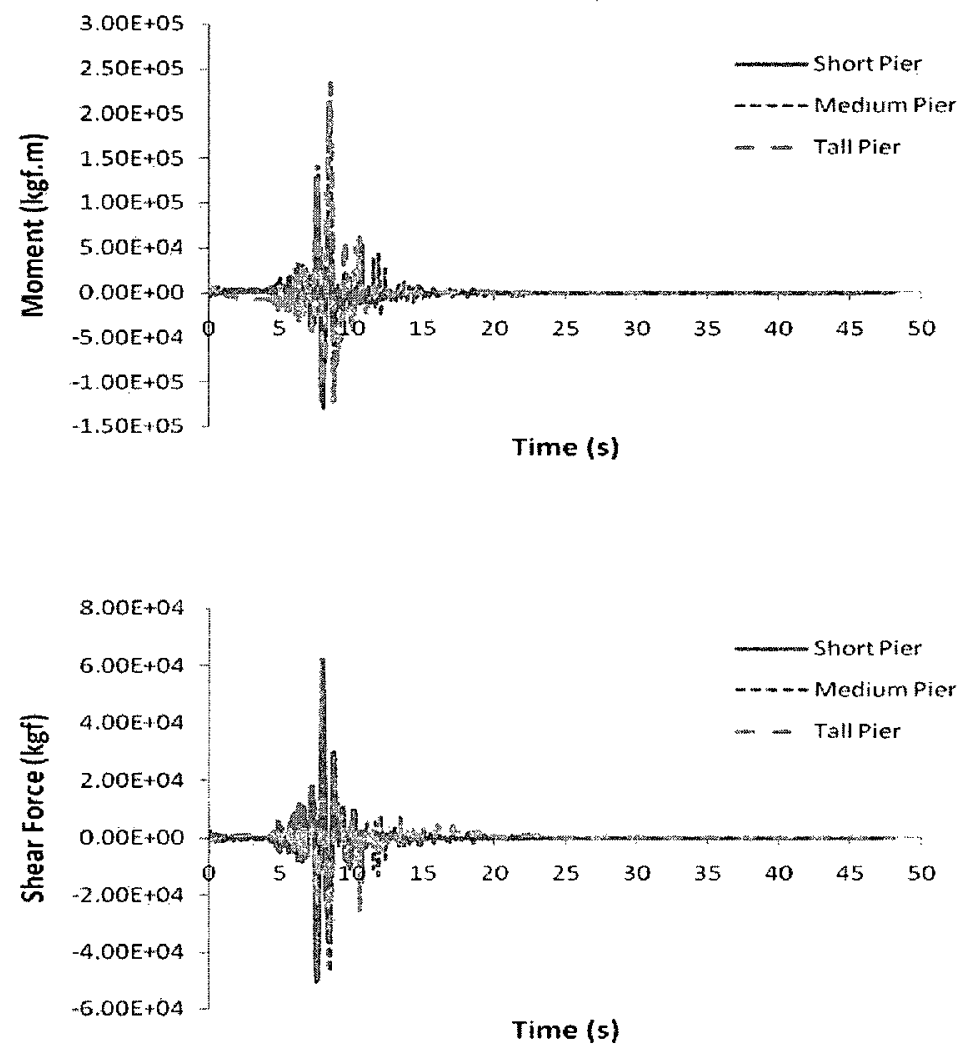

(b)

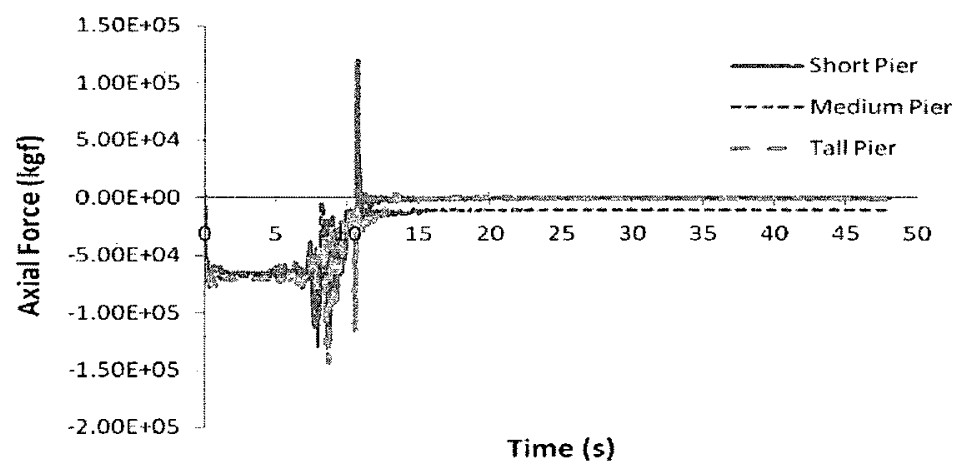

(c)

Figure 5.33. (a) Moment, (b) Shear Force, and (c) Axial Force Time Histories at the Base of the Piers due to the 1995 Kobe Earthquake Excitation 


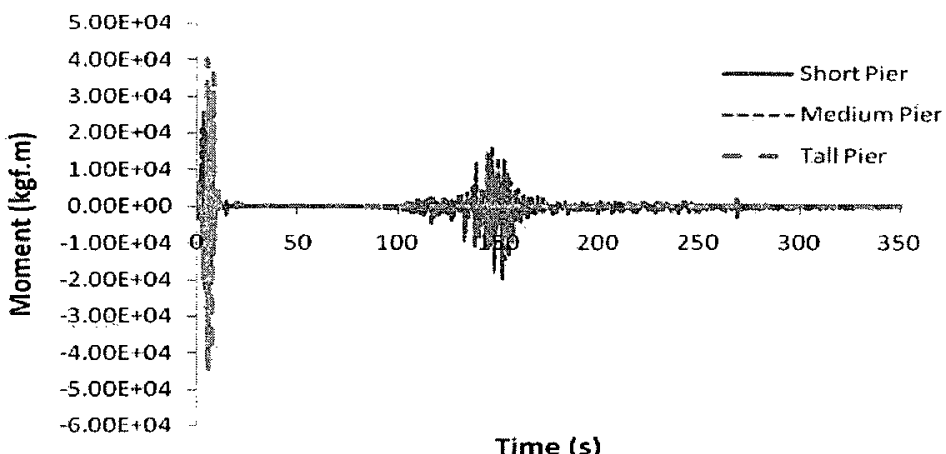

(a)

Time (s)

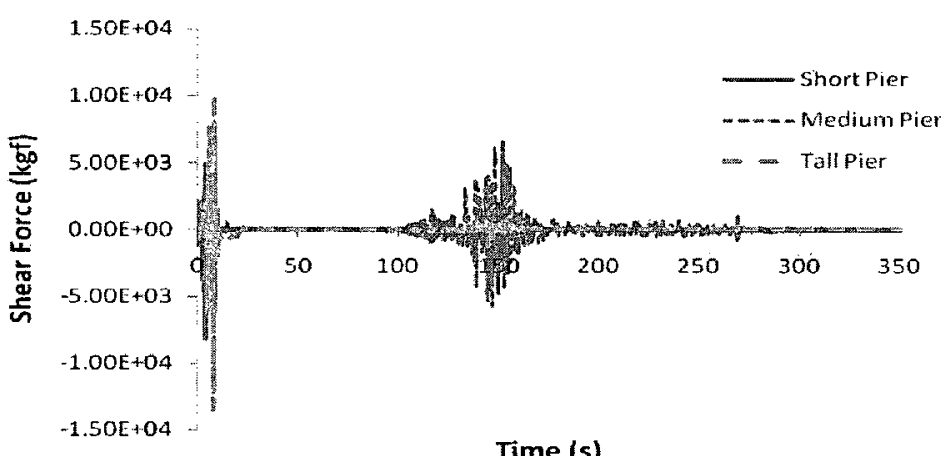

(b)

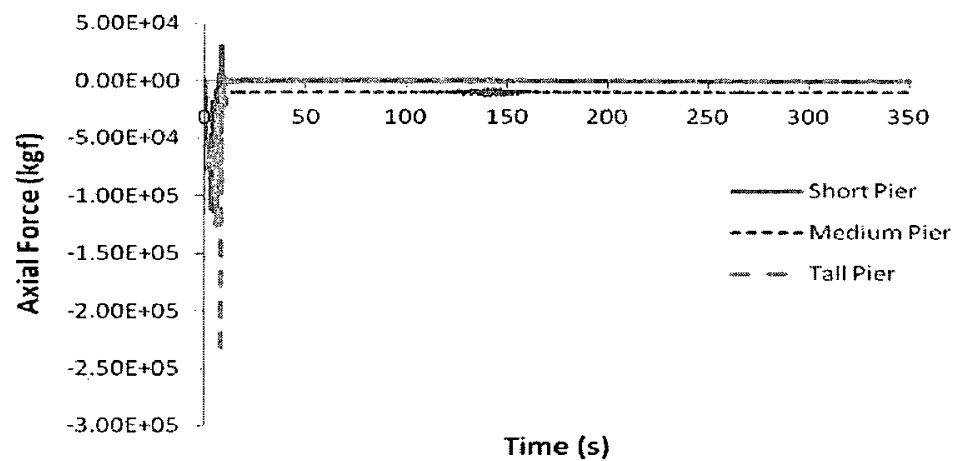

(c)

Figure 5.34. (a) Moment, (b) Shear Force, and (c) Axial Force Time Histories at the Base of the Piers due to the 1999 Chi-Chi Earthquake Excitation 
(a)

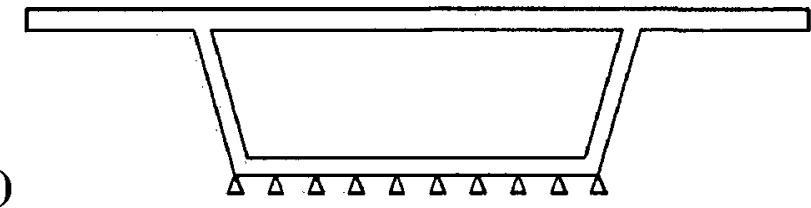

(b)

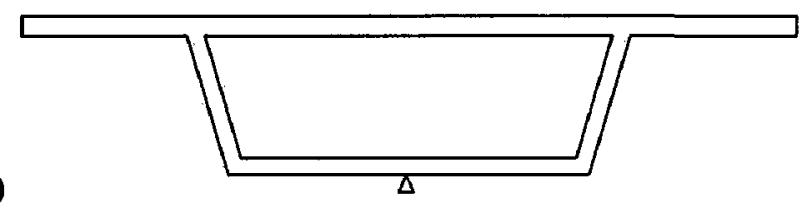

(c)

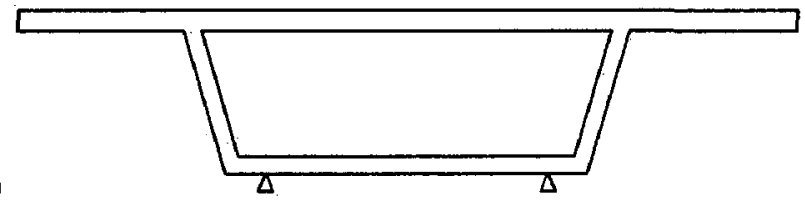

Figure 5.35. Types of Abutments: (a) A1, (b) A2, and (c) A3 


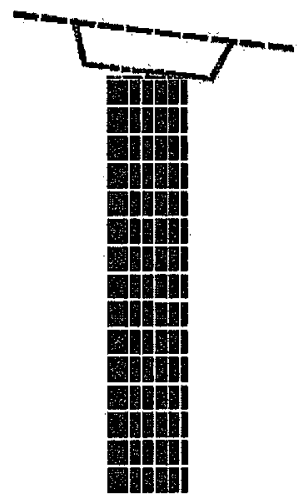

Figure 5.36. Box Girder Rocking Modal Shape 


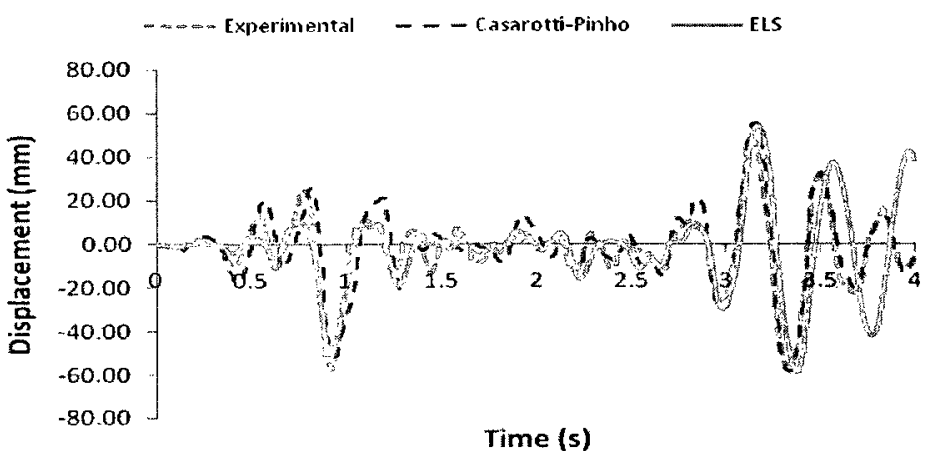

(a)

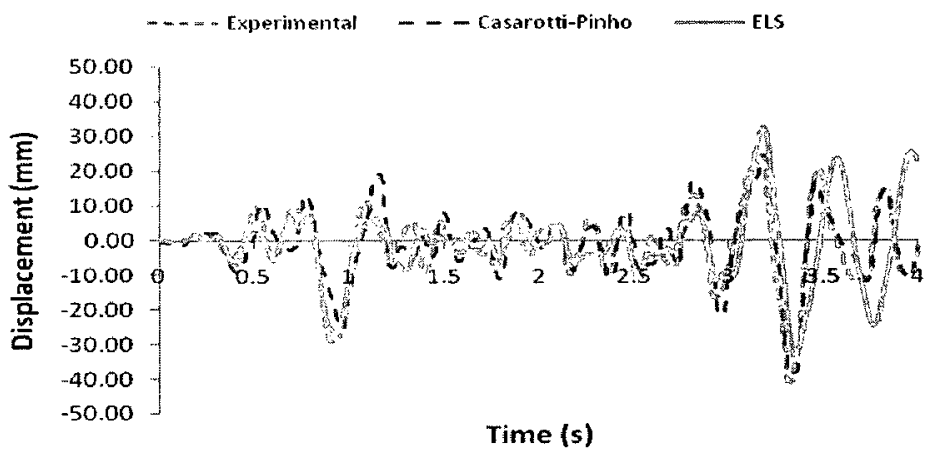

(b)

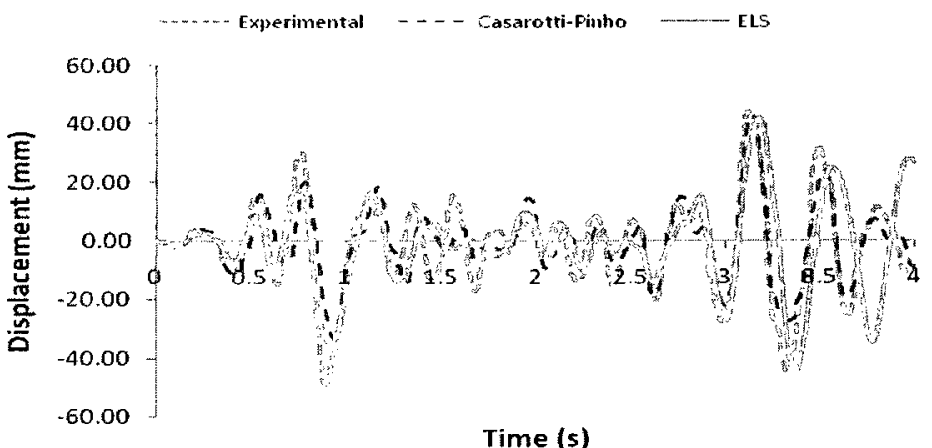

(c)

Figure 5.37. Comparisons of (a) Short, (b) Medium, and (c) Tall Piers' Displacements with Elastic Superstructure Model 


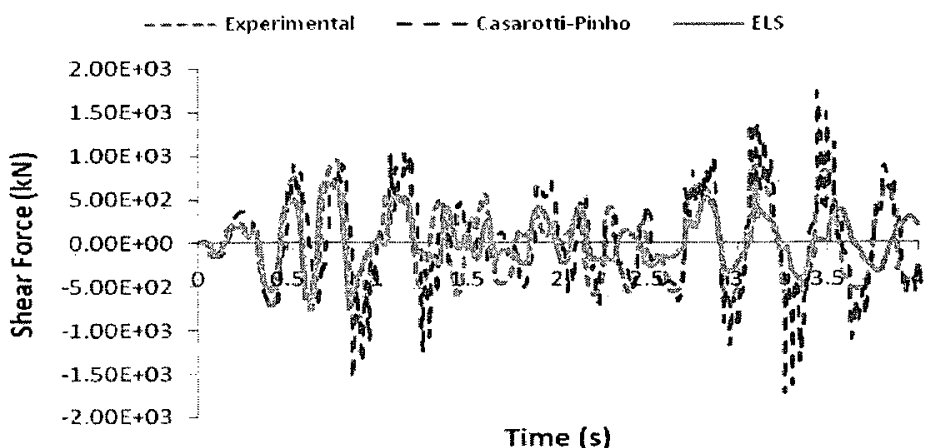

(a)

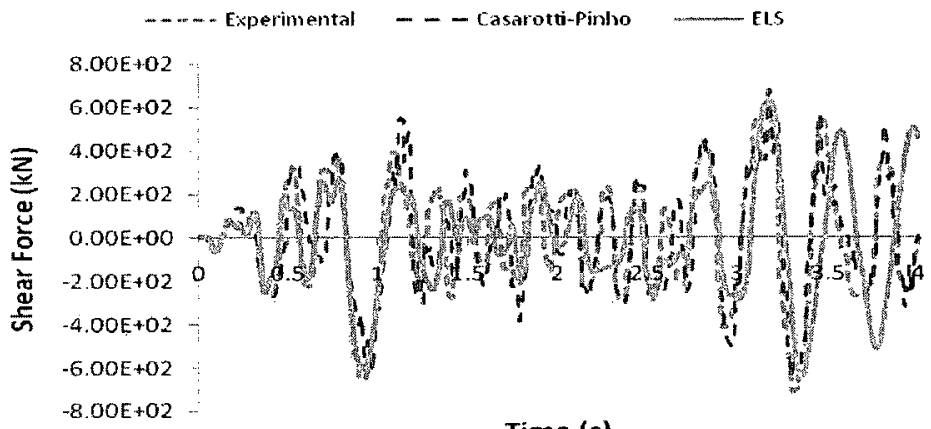

(b)

Time (s)

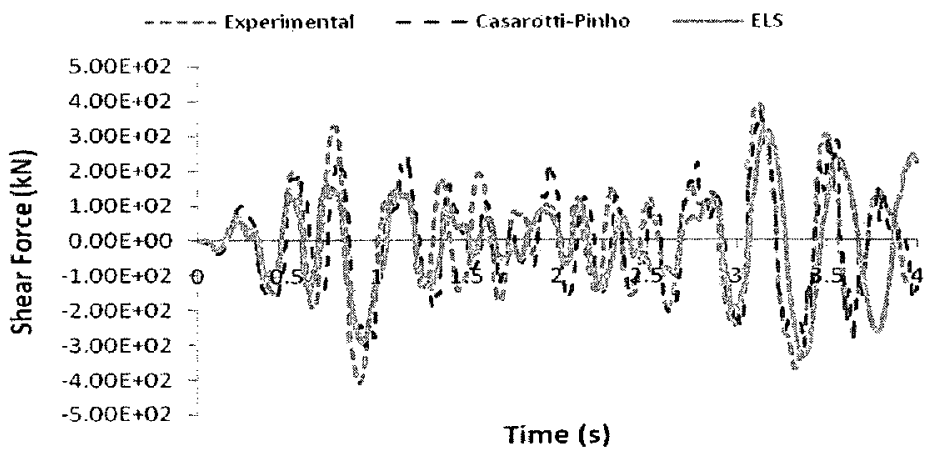

(c)

Figure 5.38. Comparisons of (a) Short, (b) Medium, and (c) Tall Piers' Top Shear Forces with Elastic Superstructure Model 
ธั่

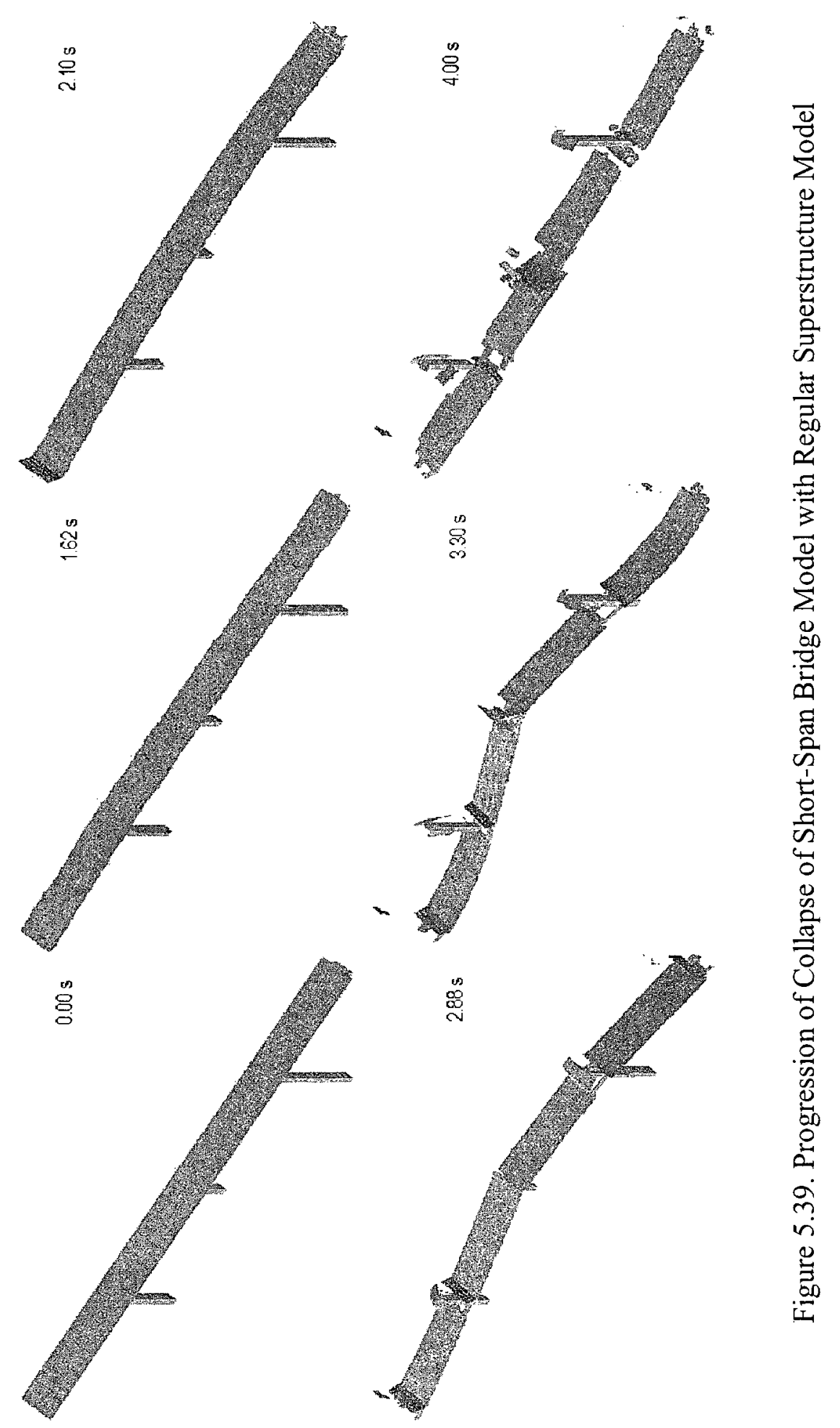


(a)
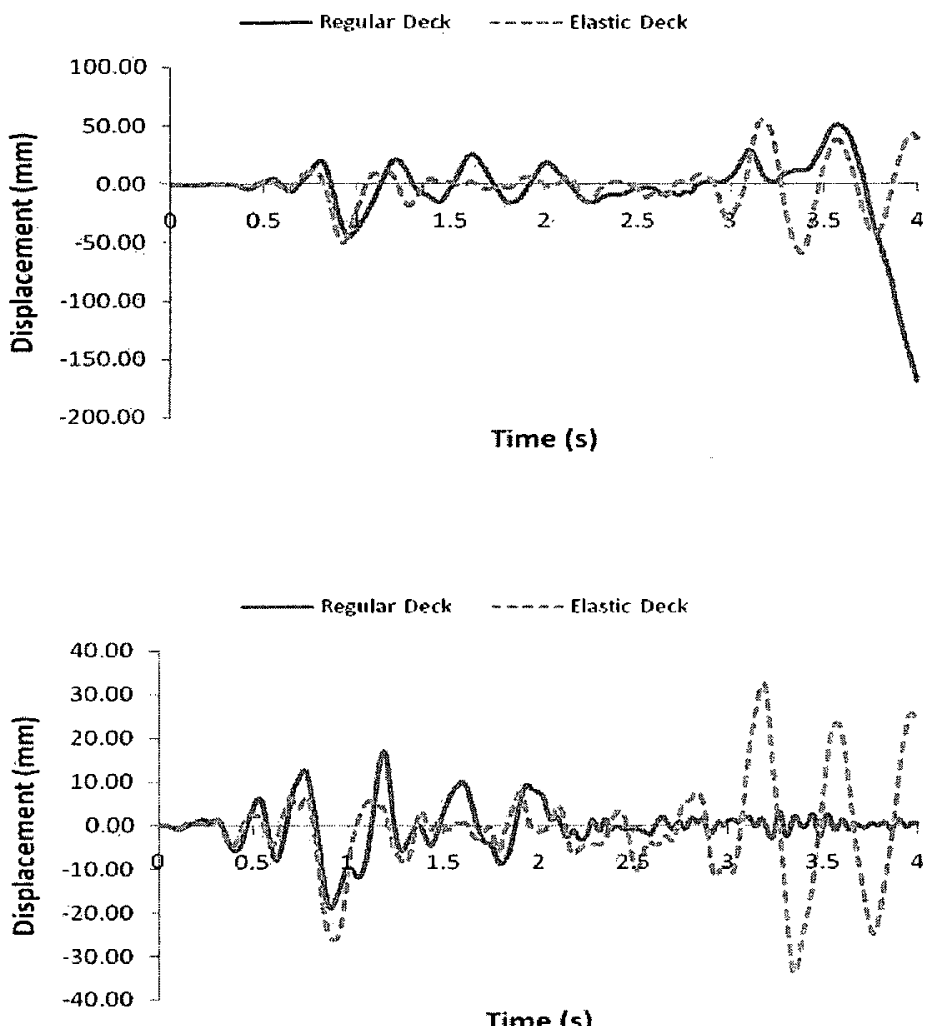

(b)

Time (s)

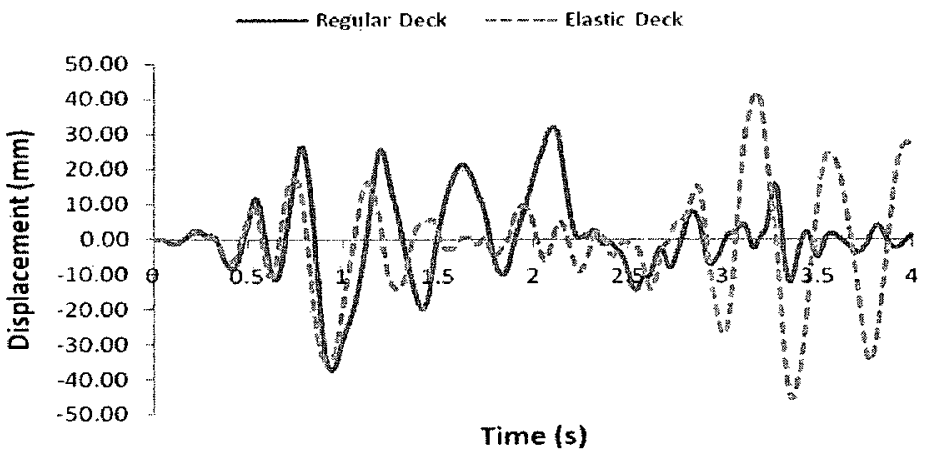

(c)

Figure 5.40. Comparisons of (a) Short, (b) Medium, and (c) Tall Piers' Displacements with Regular Superstructure Model 
(a)

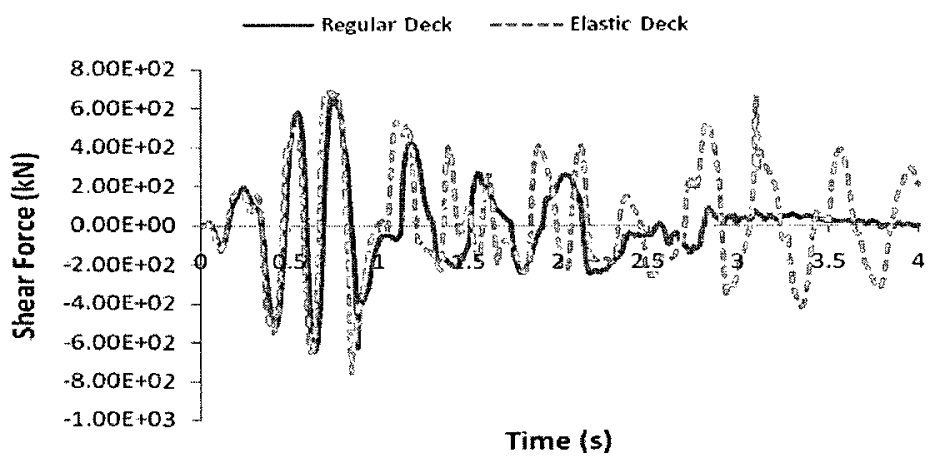

(b)

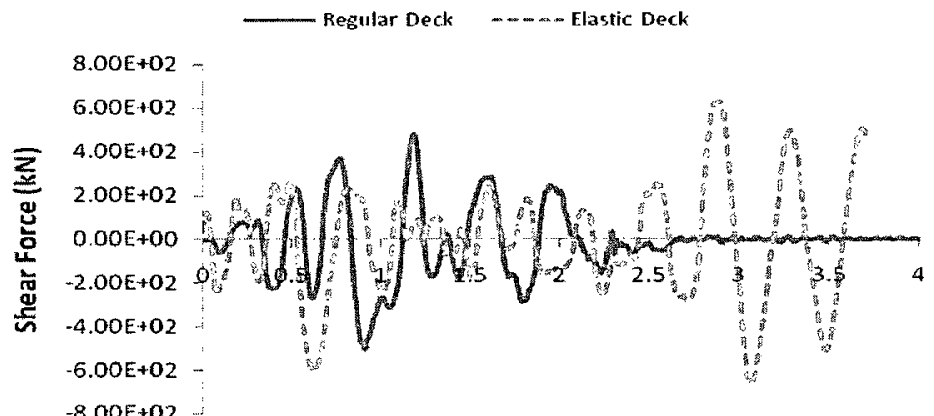

Time (s)

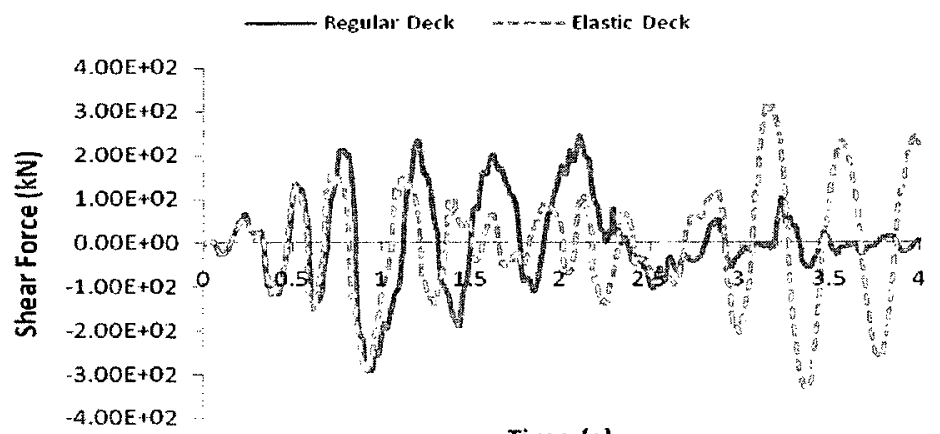

(c)

rime (s)

Figure 5.41. Comparisons of (a) Short, (b) Medium, and (c) Tall Piers' Top Shear Forces with Regular Superstructure Model 


\section{CHAPTER 6}

\section{Conclusions and Recommendations}

This chapter presents conclusions of the investigation on progressive collapse analysis and behaviour of reinforced concrete bridges and gives some suggestions for future work. In the conclusion part, a summary of the findings are given on the validity of the AEM implemented in the software ELS for simulating the progressive collapse behaviour of bridges.

\subsection{Concluding Remarks}

In this study, the seismic progressive collapse behaviour and analysis of reinforced concrete bridges are evaluated. One short-span and one medium-span reinforced concrete bridges are used as the analyses models. The bridge models and selected earthquake excitations used in the study are discussed. Some important factors that can influence the progressive collapse responses of bridges during earthquakes are investigated. In addition, an overview of the current design guideline, code, and standard requirements also analytical tools for progressive collapse resistant design of structures are presented. A summary of the findings are presented herein. 


\subsubsection{Findings on Seismic Progressive Collapse of Bridges}

- Similar progressive collapse phenomena observed in collapse of building structures caused by blast or abnormal loads are also observed in the failures of structures during earthquakes although the damage and propagation characteristics are more global in nature, as can be observed in the analysis and simulation results in this study.

- There are some general guideline recommendations and design code and standard requirements related to progressive collapse resistant design of buildings; comparatively, there are relatively few design requirements and recommendations for bridges against progressive collapse failure.

- During a severe earthquake, after the initial failure of individual structural members due to yielding of reinforcing steel and/or crushing of concrete, the repeated inelastic cyclic responses and the accumulated damage effects of repeated inelastic load reversals and large displacement demands will result in degradation of the stiffness and strength of the individual members and the structural system as a whole. 
Collapse of even only a single structural component in a bridge can have significant ramification effect that can drastically change the behaviour of the entire bridge system.

The response of structures during the collapse process is highly nonlinear and influenced by the response from impact force of falling debris; there are drastic changes in mass, strength, and stiffness properties of the structure during the progressive collapse process.

Sufficient ductility capacity in bridge columns or piers helps to ensure ductile behaviour and higher deformation capability of the structure that leads to better performance against seismic progressive collapse.

- $\quad$ Although the pier in this study has suffered damages and permanent deformation it can still effectively carry the axial compressive loads from the bridge superstructure and its own self-weight; damage reduces the pier shear capacity and lateral load resistance of the bridge structural system.

- $\quad$ Since the bridge softens due to strength or stiffness deteriorations after structural members start to fail and suffer damage, the period of the structure becomes longer due to its softening. This makes it more vulnerable to more severe earthquake damage in the case of 
the 1999 Chi-Chi earthquake which has higher seismic energy in the long period range compared to other earthquake records in this study.

Seismic progressive collapse simulations can help structural engineers to decide the best retrofit strategies for old and deficient bridge stock considering the severity of the damage in the structure.

Seismic progressive collapse simulations can help structural engineers to better understand the design objective in performancebased seismic design of structures, including a more comprehensive approach in devising effective retrofit strategies for old deficient bridges by considering the pattern and severity of the potential damage in the structure.

\subsubsection{Findings on the Applicability of the Applied Element Method for Seismic Progressive Collapse Analysis of Structures}

- The AEM procedure implemented in the software ELS can accurately simulate the seismic progressive collapse behaviour of structures and is able to predict the initiation of collapse, progression of collapse, and the final collapse mechanisms. 
- $\quad$ The AEM procedure in the software ELS allows separation of structural members or components due to fracture failure, and takes into account the effects of contact and inertial forces caused by the falling debris in the dynamic responses of structures.

The AEM procedure in the software ELS can follow the internal forces redistribution during failure of a structural component, which can be significant in a progressive collapse event.

It is shown that the software ELS gives good results in the area of linear, nonlinear, and collapse analyses of structures.

\subsection{Suggestions for Future Works}

In order to continue the study of seismic progressive collapse of bridges, several suggestions are presented hereafter. There are some important fields along this direction that deserves further study to obtain better understanding on the topic. Several suggestions for the development of the software ELS are also presented to improve the capability of this software for seismic progressive collapse analysis purposes. 


\title{
6.2.1. Further Research on Seismic Progressive Collapse of Bridges
}

\author{
Further study on bridges with different number of spans, \\ particularly to distinguish between odd and even numbers, are \\ required to justify whether different number of spans can affect the \\ failure mode of the bridge.
}

- Several analyses on different configurations considering both irregularities in plan and in height of bridges can also be done, especially skewed bridge to elaborate the current findings on seismic progressive collapse of bridges.

Seismic progressive collapse analyses for different types of bridges, such as cable-stayed or truss bridges can be studied.

- The modelling of abutments and bearings can be refined and further investigated.

Seismic progressive collapse analyses of prestressed concrete or steel bridges can also be done as comparisons to reinforced concrete bridges.

The effect of soil-structure interaction, especially for long-span bridges that needs to take into account the effects of multiplesupport excitations, should also be investigated. 


\subsubsection{Further Development of the Software Extreme Loading for Structures}

- Element shapes in ELS are limited to rectangular and circular shapes, therefore it is suggested that an option of arbitrary shape is included so the modelling process will be faster.

The output parameters in ELS should also include the momentcurvature relationship and the ductility capacity of a section with their deterioration during collapse process for seismic analysis of structures.

- It is also suggested that the next version incorporates the option to obtain the modal shape vectors since the current version of ELS can only show the modal shapes but the user cannot obtain the displacement or modal shape vectors easily.

The confined concrete material model can be applied in addition to the Maekawa model currently used to refine the analysis results.

The effect of bond and rebar slips can also be taken into account to obtain better results.

Several drawbacks on the labelling, such as labelling new rebar size or custom component can be improved by giving the users option to label them and not just assigning numbers, which is harder to trace. 
- The separation between the analysis solver and viewer can lead to misinterpretation of the results since the input file could be changed but the output file still remains unchanged. 


\section{References}

American Association of State Highway and Transportation Officials, AASHTO. (2007). AASHTO LRFD Bridge Design Specifications, SI Units, $4^{\text {th }}$ Ed. Washington, DC: AASHTO.

American Concrete Institute, ACI. (2008). Building Code Requirements for Structural Concrete (ACI 318-08) and Commentary. Farmington Hills: ACI.

American Society of Civil Engineers, ASCE. (2006). Minimum Design Loads for Buildings and Other Structures, Report ASCE/SEI 7-05. New York: ASCE.

Anderson, D. L., Mitchell, D., and Tinawi, R. G. (1996). Performance of Concrete Bridges during the Hyogo-ken Nanbu (Kobe) Earthquake on January 17, 1995. Canadian Journal of Civil Engineering, 23 (3), pp. 714-726.

Applied Science International, ASI. (2006). Extreme Loading for Structures Technical Manual. Raleigh: ASI.

Areias, P. M. A. and Belytschko, T. (2005). Non-linear Analysis of Shells with Arbitrary Evolving Cracks using XFEM. International Journal for Numerical Methods in Engineering, 62 (3), pp. 384-415.

Banerjee, S. and Shinozuka, M. (2004). Dynamic Progressive Failure of Bridges. Proceedings of the $9^{\text {th }}$ ASCE Joint Specialty Conference on Probabilistic 
Mechanics and Structural Reliability, Albuquerque, NM, U.S.A., July 26-28, $6 \mathrm{pp}$.

Bao, Y., Kunnath, S. K., El-Tawil, S., and Lew, H. S. (2008). Macromodel-Based Simulation of Progressive Collapse: RC Frame Structures. Journal of Structural Engineering ASCE, 134 (7), pp. 1079-1091.

Belytschko, T., Organ, D., and Krongauz, Y. (1995). A Coupled Finite Element Element-Free Galerkin Method. Computational Mechanics, 17 (3), pp. 186195.

Buckle, I. G. (2003). Application of Seismic Risk Assessment Procedures to the Performance-Based Design of Highway Systems. Advancing Mitigation Technologies and Disaster Response for Lifeline Systems, Beavers, J. E. (ed.), pp. 886-895.

Canadian Standards Association, CSA. (2006). CAN/CSA-S6-06 Canadian Highway Bridge Design Code. Toronto: CSA International.

Casarotti, C., Pinho, R., and Calvi, G. M. (2005). Adaptive Pushover-based Methods for Seismic Assessment and Design of Bridge Structures, Research Report No. ROSE 2005/06. Pavia: IUSS Press.

Casarotti, C. and Pinho, R. (2006). Seismic Response of Continuous Span Bridges through Fiber-based Finite Element Analysis. Earthquake Engineering and Engineering Vibration, 5 (1), pp. 119-131. 
Casarotti, C. and Pinho, R. (2007). An Adaptive Capacity Spectrum Method for Assessment of Bridges Subjected to Earthquake Action. Bulletin of Earthquake Engineering, 5 (3), pp. 377-390.

Chopra, A. K. (2007). Dynamics of Structures: Theory and Applications to Earthquake Engineering, $3^{\text {rd }}$ Ed. Upper Saddle River: Prentice-Hall, Inc.

Clough, R. W. and Penzien, J. (2003). Dynamics of Structures, $3^{r d}$ Ed. Berkeley: Computers and Structures, Inc.

Corley, W. G. (2002). Applicability of Seismic Design in Mitigating Progressive Collapse. In National Workshop on Prevention of Progressive Collapse, Rosemont, IL, July 10-11. Multihazard Mitigation Council of the National Institute of Building Sciences, Washington, DC, U.S.A., 13 pp.

Cundall, P. A. (1971). A Computer Model for Simulating Progressive, Large Scale Movement in Blocky Rock System. Proceedings of the Symposium of the International Society for Rock Mechanics: Rock Fracture, Nancy, France, Vol. 1, Paper No. II-8, pp. 129-136.

Department of Defense, DoD. (2009). Design of Buildings to Resist Progressive Collapse, Unified Facilities Criteria (UFC) 4-023-03.

El-Hacha, R. and Bullock, B. (2006). Progressive Collapse for Reinforced Concrete Buildings. Proceedings of the $1^{\text {st }}$ Specialty Conference on Disaster Mitigation CSCE, Calgary, AB, Canada, May 23-26, 13 pp. 
Elwood, K. J. and Moehle, J. P. (2003). Shake Table Tests and Analytical Studies on the Gravity Load Collapse of Reinforced Concrete Frames, Report PEER 2003/01. Berkeley: PEER Center.

Elwood, K. J. and Moehle, J. P. (2008). Dynamic Collapse Analysis for a Reinforced Concrete Frame Sustaining Shear and Axial Failure. Earthquake Engineering and Structural Dynamics, 37 (7), pp. 991-1012.

Federal Emergency Management Agency, FEMA. (1996). The Oklahoma City Bombing: Improving Building Performance through Multi-hazard Mitigation, Report FEMA 277. Washington, DC: FEMA.

General Services Administration, GSA. (2003). Progressive Collapse Analysis and Design Guidelines for New Federal Office Buildings and Major Modernizations Projects.

Ghali, A. and Tadros, G. (1997). Bridge Progressive Collapse Vulnerability. Journal of Structural Engineering ASCE, 123 (2), pp. 227-231.

Ghali, A. and Tadros, G. (1998). Closure: Bridge Progressive Collapse Vulnerability. Journal of Structural Engineering ASCE, 124 (12), p. 1498.

Grierson, D. E., Safi, M., Xu, L., and Liu, Y. (2005). Simplified Methods for Progressive-Collapse Analysis of Buildings. Proceedings of ASCE 2005 Structures Congress: Metropolis and Beyond, New York, NY, U.S.A., April $20-24,8 \mathrm{pp}$. 
Griffiths, H., Pugsley, A., and Saunders, O. (1968). Report of Inquiry into the Collapse of Flats at Ronan Point, Canning Town. London: Ministry of Housing and Local Government, Her Majesty's Stationary Office.

Gross, J. L. and McGuire, W. (1983). Progressive Collapse Resistant Design. Journal of Structural Engineering ASCE, 109 (1), pp. 1-15.

Guedes, J. (1997). Seismic Behavior of Reinforced Concrete Bridges: Modelling, Numerical Analysis, and Experimental Assessment. Ph.D. Thesis, Department of Civil Engineering, University of Porto, Portugal.

Gurley, C. (2008). Progressive Collapse and Earthquake Resistance. Practice Periodical on Structural Design and Construction ASCE, 13 (1), pp. 19-23.

Hakuno, M. and Meguro, K. (1993). Simulation of Concrete-Frame Collapse due to Dynamic Loading. Journal of Engineering Mechanics ASCE, 119 (9), pp. 1709-1723.

Han, Q., Du, X., Liu, J., Li, Z., Li, L., and Zhao, J. (2009). Seismic Damage of Highway Bridges during the 2008 Wenchuan Earthquake. Earthquake Engineering and Engineering Vibration, 8 (2), pp. 263-273.

Haselton, C. B., Liel, A. B., Dean, B. S., Chou, J. H., and Deierlein, G. G. (2007). Seismic Collapse Safety and Behavior of Modern Reinforced Concrete Moment Frame Buildings. Proceedings of ASCE 2007 Structures Congress: New Horizons Better Practices, Long Beach, CA, U.S.A., May 16-19, 14 pp. 
Hayes, J. R., Jr., Woodson, S. C., Pekelnicky, R. G., Poland, C. D., Corley, W. G., and Sozen, M. (2005). Can Strengthening for Earthquake Improve Blast and Progressive Collapse Resistance?. Journal of Structural Engineering ASCE, $131(8)$, pp. 1157-1177.

Hillerborg, A., Modéer, M., and Petersson, P. -E. (1976). Analysis of Crack Formation and Crack Growth in Concrete by Means of Fracture Mechanics and Finite Elements. Cement and Concrete Research, 6, pp. 773-782.

Hsu, Y. T. and Fu, C. C. (2004). Seismic Effect on Highway Bridges in Chi Chi Earthquake. Journal of Performance of Constructed Facilities ASCE, 18 (1), pp. 47-53.

Humar, J. L. (2002). Dynamics of Structures, $2^{\text {nd }}$ Ed. Leiden: Taylor \& Francis / Balkema.

Ibarra, L. F. and Krawinkler, H. (2005). Global Collapse of Frame Structures under Seismic Excitations, Report PEER 2005/06. Berkeley: PEER Center.

Ibarra, L. F., Medina, R. A., and Krawinkler H. (2005). Hysteretic Models that Incorporate Strength and Stiffness Deterioration. Earthquake Engineering and Structural Dynamics, 34 (12), pp. 1489-1511.

Ioani, A. M., Cucu, H. L., and Mircea, C. (2007). Seismic Design vs. Progressive Collapse: A Reinforced Concrete Framed Structure Case Study. Proceedings of the $4^{\text {th }}$ International Structural Engineering and Construction Conference: 
Innovations in Structural Engineering and Construction, Xie, M. and Patnaikuni, I. (eds.), Melbourne, Australia, 26-28 September, pp. 955-961.

Isobe, D. and Tsuda, M. (2003). Seismic Collapse Analysis of Reinforced Concrete Framed Structures using the Finite Element Method. Earthquake Engineering and Structural Dynamics, 32 (13), pp. 2027-2046.

Jennings, P. C. and Husid, R. (1968). Collapse of Yielding Structures during Earthquakes. Journal of the Engineering Mechanics Division ASCE, 94 (EM5), pp. 1045-1065.

Johnson, N. S. (2006). Large-Scale Experimental and Analytical Seismic Studies of a Two-Span Reinforced Concrete Bridge System. Ph.D. Thesis, University of Nevada, Reno, U.S.A.

Kaewkulchai, G. and Williamson, E. B. (2002). Dynamic Progressive Collapse of Frame Structures. Proceedings of $15^{\text {th }}$ ASCE Engineering Mechanics Conference, New York, NY, U.S.A., June 2-5, 7 pp.

Kaewkulchai, G. and Williamson, E. B. (2004). Beam Element Formulation and Solution Procedure for Dynamic Progressive Collapse Analysis. Computers and Structures, 82 (7-8), pp. 639-651.

Kawai, T. (1980). Some Considerations on the Finite Element Method. International Journal for Numerical Methods in Engineering, 16 (1), pp. 81-120. 
Kawashima, K. (2000). Seismic Performance of RC Bridge Piers in Japan: An Evaluation After the 1995 Hyogo-ken Nanbu Earthquake. Progress in Structural Engineering and Materials, 2 (1), pp. 82-91.

Kikuchi, A., Kawai, T., and Suzuki, N. (1992). The Rigid Bodies-Spring Models and Their Applications to Three Dimensional Crack Problems, Computers \& Structures, 44 (1-2), pp. 469-480.

Kim, H. -S., Kim, J., and An, D. -W. (2009). Development of Integrated System for Progressive Collapse Analysis of Building Structures Considering Dynamic Effects. Advances in Engineering Software, 40 (1), pp. 1-8.

Kramer, S. L. (1996). Geotechnical Earthquake Engineering. Upper Saddle River: Prentice Hall, Inc.

Krawinkler, H. and Seneviratna, G. D. P. K. (1998). Pros and Cons of a Pushover Analysis of Seismic Performance Evaluation. Engineering Structures, 20 (4), pp. $452-464$.

Kupfer, H., Hilsdorf, H. K., and Rusch, H. (1969). Behavior of Concrete under Biaxial Stresses, ACI Journal, 66 (8), pp. 656-666.

Lu, X., Lin, X., Ma, Y., and Ye, L. (2008). Numerical Simulation for the Progressive Collapse of Concrete Building due to Earthquake. Proceedings of the $14^{\text {th }}$ World Conference on Earthquake Engineering, Beijing, China, October 12$17,8 \mathrm{pp}$. 
Lu, X., Lin, X., and Ye, L. (2009). Simulation of Structural Collapse with Coupled Finite Element - Discrete Element Method. Proceedings of International Symposium on Computational Structural Engineering, Yuan, Y., Cui, J. Z., and Mang, H. (eds.), Shanghai, China, June 22-24, 8 pp.

Marjanishvili, S. M. (2004). Progressive Analysis Procedure for Progressive Collapse. Journal of Performance of Constructed Facilities ASCE, 18 (2), pp. 79-85.

Marjanishvili, S. and Agnew, E. (2005). Comparison of Various Procedures for Progressive Collapse Analysis. Journal of Performance of Constructed Facilities ASCE, 20 (4), pp. 365-374.

Meguro, K. and Hakuno, M. (1989). Fracture Analyses of Concrete Structures by the Modified Distinct Element Method. Structural Engineering/Earthquake Engineering JSCE, 6 (2), pp. 283s-294s.

Meguro, K. and Hakuno, M. (1992). Simulation of Collapse due to Earthquakes Using the Extended Distinct Element Method. Proceedings of the $10^{\text {th }}$ World Conference on Earthquake Engineering, Madrid, Spain, July 19-24, pp. 3793-3796.

Meguro, K. and Tagel-Din, H. (1999). Simulation of Buckling and Post-buckling Behavior of Structures using Applied Element Method. Bulletin of Earthquake Resistant Structure Research Center, 32, pp. 125-135. 
Meguro, K. and Tagel-Din, H. (2000). Applied Element Method for Structural Analysis: Theory and Application for Linear Materials. Structural Engineering/Earthquake Engineering JSCE, 17 (1), pp. 21s-35s.

Meguro, K. and Tagel-Din, H. (2001). Applied Element Simulation of RC Structures under Cyclic Loading. Journal of Structural Engineering ASCE, 127 (11), pp. 1295-1305.

Meguro, K. and Tagel-Din, H. S. (2002). Applied Element Method Used for Large Displacement Structural Analysis. Journal of Natural Disaster Science, 24 (1), pp. 25-34.

Menchel, K., Massart, T. J., Rammer, Y., and Bouillard, P. (2009). Comparison and Study of Different Progressive Collapse Simulation Techniques for RC Structures. Journal of Structural Engineering ASCE, 135 (6), pp. 685-697.

Miao, Z. W., Lu, X. Z., Ye, L. P., and Ma, Q. L. (2007). Simulation for the Collapse of RC Frame Tall Buildings under Earthquake Disaster. Proceedings of International Symposium on Computational Mechanics 2007, Yao, Z. H. and Yuan, M. W. (eds.), Beijing, China, July 30 - August 1, 6 pp.

Mitchell, D., Bruneau, M., Williams, M., Anderson, D., Saatcioglu, M., and Sexsmith, R. (1995). Performance of Bridges in the 1994 Northridge Earthquake. Canadian Journal of Civil Engineering, 22 (2), pp. 415-427. 
Moehle, J. P., Elwood, K. J., and Sezen, H. (2002). Gravity Load Collapse of Building Frames during Earthquakes. Proceedings of S. M. Uzumeri Symposium: Behavior and Design of Concrete Structures for Seismic Performance, ACI Special Publication (SP-197), pp. 215-238.

Moehle, J. P. and Eberhard, M. O. (2003). Earthquake Damage to Bridges. Bridge Engineering: Seismic Design, Chen, W. -F. and Duan, L. (eds.), 33 pp.

Moehle, J. P., Ghannoum, W., and Bozorgnia, Y. (2006). Collapse of Lightly Confined Reinforced Concrete Frames during Earthquake. Advances in Earthquake Engineering for Urban Risk Reduction, Wasti, S. T. and Ozcebe, G. (eds.), pp. 317-332.

Moës, N. and Belytschko, T. (2002). Extended Finite Element Method for Cohesive Crack Growth. Engineering Fracture Mechanics, 69 (7), pp. 813-833.

Mosalam, K. M., Talaat, M., and Park, S. (2008). Modeling Progressive Collapse in Reinforced Concrete Framed Structures. Proceedings of the $14^{\text {th }}$ World Conference on Earthquake Engineering, Beijing, China, October 12-17, 8 pp.

Munjiza, A. (2004). The Combined Finite - Discrete Element Method. Chichester: John Wiley \& Sons, Ltd.

Nair, R. S. (2006). Preventing Disproportionate Collapse. Journal of Performance of Constructed Facilities ASCE, 20 (4), pp. 309-314. 
Nakamura, T. and Yoshimura, M. (2002). Gravity Load Collapse of Reinforced Concrete Columns with Brittle Failure Modes. Journal of Asian Architecture and Building Engineering, 1 (1), pp. 21-27.

National Institute of Standards and Technology, NIST. (2005). Final Report on the Collapse of the World Trade Center Tower, Report NCSTAR 1. Gaithersburg: NIST.

National Institute of Standards and Technology, NIST. (2007). Best Practices for Reducing the Potential of Progressive Collapse in Buildings, Report NISTIR 7396. Gaithersburg: NIST.

National Research Council of Canada, NRCC. (2005). National Building Code of Canada 2005. Ottawa: NRCC.

Newmark, N. M. (1959). A Method of Computation for Structural Dynamics. Journal of the Engineering Mechanics Division ASCE, 85 (EM3), pp. 67-94.

Okamura, H. and Maekawa, K. (1991). Nonlinear Analysis and Constitutive Models of Reinforced Concrete. Tokyo: Gihodo Co. Ltd.

Phung, V. (2005). Strong Ground Motions for Bridge Design and Non-Linear Dynamic Response Analysis of Bridges. Ph.D. Thesis, Department of Civil and Environmental Engineering, Carleton University, Canada.

Phung, V. and Lau, D. T. (2006). A 3D Stiffness Degradation Model for Earthquake Analyses of Bridges. Proceedings of the $8^{\text {th }} U . S$. National Conference on 
Earthquake Engineering, San Fransisco, CA, U.S.A., April 18-22, Paper No. $1112,10 \mathrm{pp}$.

Powell, G. (2005). Progressive Collapse: Case Studies Using Nonlinear Analysis. Proceedings of ASCE 2005 Structures Congress: Metropolis and Beyond, New York, NY, U.S.A., April 20-24, 14 pp.

Pretlove, A. J., Ramsden, M., and Atkins, A. G. (1991). Dynamic Effects in Progressive Failure of Structures. International Journal of Impact Engineering, 11 (4), pp. 539-546.

Priestley, M. J. N., Seible, F., and Calvi, G. M. (1996). Seismic Design and Retrofit of Bridges. New York: John Wiley \& Sons, Inc.

Ristic, D., Yamada, Y., and Iemura, H. (1986). Stress-strain Based Modeling of Hysteretic Structures under Earthquake Induced Bending and Varying Axial Loads, Research Report No. 86-ST-01, School of Civil Engineering, Kyoto University, Kyoto, Japan.

Seible, F., Hegemier, G., Karbhari, V. M., Wolfson, J., Arnett, K., Conway, R., and Baum, J. D. (2008). Protection of Our Bridge Infrastructure against Manmade and Natural Hazards. Structure and Infrastructure Engineering, 4 (6), pp. $415-429$.

Sezen, H. (2000). Evaluation and Testing of Existing Reinforced Concrete Building Columns, CE299 Report. Berkeley: University of California, Berkeley. 
Shinozuka, M., Banerjee, S., and Kim, S. -H. (2007). Fragility Considerations in Highway Bridge Design, Technical Report MCEER-07-0023. Buffalo: MCEER.

Sivaselvan, M. V. and Reinhorn, A. M. (2006). Lagrangian Approach to Structural Collapse Simulation. Journal of Engineering Mechanics ASCE, 132 (8), pp. 795-805.

Smilowitz, R. (2002). Analytical Tools for Progressive Collapse Analysis. Proceedings of National Workshop on Prevention of Progressive Collapse, Multihazard Mitigation Council of the National Institute of Building Sciences, Rosemont, IL, U.S.A., July 10-11, 9 pp.

Starossek, U. (2006). Progressive Collapse of Bridges - Aspects of Analysis and Design. Proceedings of International Symposium on Sea-Crossing LongSpan Bridges, Mokpo, Korea, February 15-17, 22 pp.

Starossek, U. (2007). Typology of Progressive Collapse. Engineering Structures, 29 (9), pp. 2302-2307.

Starossek, U. and Sauvageot, G. (1998). Discussion: Bridge Progressive Collapse Vulnerability. Journal of Structural Engineering ASCE, 124 (12), pp. 14971498.

Stevens, D., Crowder, B., Hall, B., and Marchand, K. (2008). Unified Progressive Collapse Design Requirements for DoD and GSA. Proceedings of ASCE 
2008 Structures Congress: Crossing Borders, Vancouver, BC, Canada, April 24-26, $10 \mathrm{pp}$.

Sukumar, N., Moës, N., Moran, B., and Belytschko, T. (2000). Extended Finite Element Method for Three-Dimensional Crack Modelling. International Journal for Numerical Methods in Engineering, 48 (11), pp. 1549-1570.

Sun, L., Zhou, C., Qin, D., and Fan, L. (2003). Application of Extended Distinct Element Method with Lattice Model to Collapse Analysis of RC Bridges. Earthquake Engineering and Structural Dynamics, 32 (8), pp. 1217-1236.

Tagel-Din, H. and Meguro, K. (1999). Applied Element Simulation for Collapse Analysis of Structures. Bulletin of Earthquake Resistant Structure Research Center, Institute of Industrial Science, University of Tokyo, 32, $11 \mathrm{pp}$.

Tagel-Din, H. and Meguro, K. (2000a). Applied Element Method for Simulation of Nonlinear Materials: Theory and Application for RC Structures. Structural Engineering/Earthquake Engineering JSCE, 17 (2), pp. 137s-148s.

Tagel-Din, H. and Meguro, K. (2000b). Applied Element Method for Dynamic Large Deformation Analysis of Structures. Structural Engineering/Earthquake Engineering JSCE, 17 (2), pp. 215s-224s.

Talaat, M. M. and Mosalam, K. M. (2008). Computational Modeling of Progressive Collapse in Reinforced Concrete Frame Structures, Report PEER 2007/10. Berkeley: PEER Center. 
Taylor, D. A. (1975). Progressive Collapse. Canadian Journal of Civil Engineering, 2 (4), pp. 517-529.

Toi, Y. and Isobe, D. (1993). Adaptively Shifted Integration Technique for Finite Element Collapse Analysis of Framed Structures. International Journal for Numerical Methods in Engineering, 36 (14), pp. 2323-2339.

Ueda, M. and Kambayashi, A. (1993). Size Effect Analysis using RBSM with Vornori Elements, Proceedings of the Japan Concrete Institute International Workshop on Size Effect in Concrete Structures, Sendai, Japan, October 31 November 2, pp. 199-210.

Wallace, J. W., Eberhard, M. O., Hwang, S. -J., Moehle, J. P., Post, T., Roblee, C., Stewart, J. P., and Yashinsky, M. (2001). Highway Bridges. Earthquake Spectra: Chi-Chi Earthquake Reconnaissance Report, 17 (S1), pp. 131-152.

Wibowo, H., Reshotkina, S. S., and Lau, D. T. (2009). Modelling Progressive Collapse of RC Bridges during Earthquakes. Proceedings of CSCE Annual General Conference 2009: On the Leading Edge, St. John's, NL, Canada, May 27-30, Paper No. GC-176, 11 pp.

Yi, W. -J., He, Q. -F., Xiao, Y., and Kunnath, S. K. (2008). Experimental Study on Progressive Collapse-Resistant Behavior of Reinforced Concrete Frame Structures. ACI Structural Journal, 105 (4), pp. 433-439. 